\title{
Modelling Bottom Stress in Depth-Averaged Flows
}

\section{GC

by

Harry Leonard Jenter, II

B.S., University of Michigan (1983)

M.S.C.E., Massachusetts Institute of Technology (1987)

Submitted in partial fulfillment of the

requirements for the degree of

Doctor of Philosophy

at the

MASSACHUSETTS INSTITUTE OF TECHNOLOGY

and the

WOODS HOLE OCEANOGRAPHIC INSTITUTION

May 1989

MARINE

BIOLOGICAL

LABORATORY

LIBRARY

WOODS HOLE, MASS.

W. H. O. I.

(c) Harry L. Jenter, 1989

The author hereby grants to MIT permission to reproduce and to distribute copies of this thesis document in whole or in part.

Joint Program in Oceanographic Engineering Massachusetts Institute of Technology Woods Hole Oceanographic Institution May 19, 1989

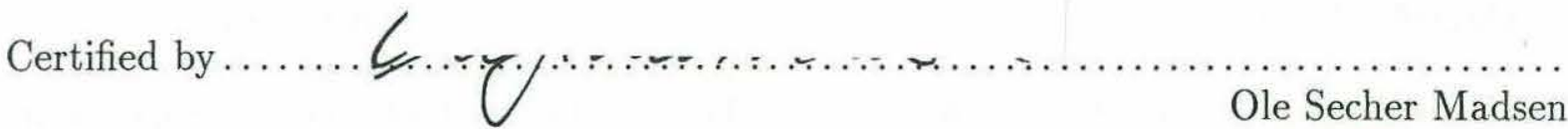

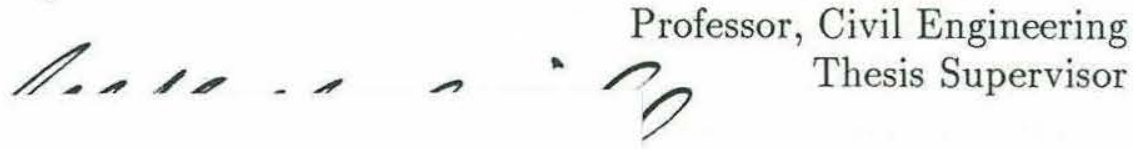

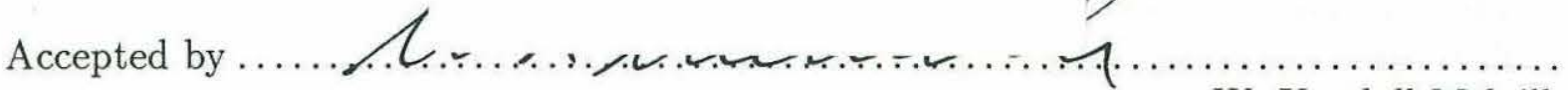

W. Kendall Melville Chairman, Joint Committee for Oceanographic Engineering, MIT/WHOI Joint

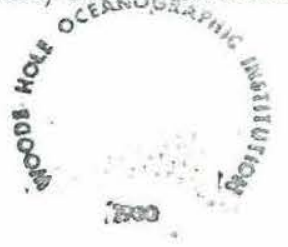

Program in Oceanographic Engineering 


\title{
Modelling Bottom Stress in Depth-Averaged Flows
}

\author{
by \\ Harry Leonard Jenter, II
Submitted to the Massachusetts Institute of Technology-
Woods Hole Oceanographic Institution
Joint Program in Oceanographic Engineering
on May 19, 1989, in partial fulfillment of the
requirements for the degree of
Doctor of Philosophy

\begin{abstract}
The relationship between depth-averaged velocity and bottom stress for wind-driven flow in unstratified coastal waters is examined here. The adequacy of traditional linear and quadratic drag laws is addressed by comparison with a $2 \frac{1}{2}$-D model. A $2 \frac{1}{2}$-D model is one in which a simplified 1-D depth-resolving model (DRM) is used to provide an estimate of the relationship between the flow and bottom stress at each grid point of a depth-averaged model (DAM). Bottom stress information is passed from the DRM to the DAM in the form of drag tensor with two components: one which scales the flow and one which rotates it. This eliminates the problem of traditional drag laws requiring the flow and bottom stress to be collinear. In addition, the drag tensor field is updated periodically so that the relationship between the velocity and bottom stress can be time-dependent. However, simplifications in the $2 \frac{1}{2}$-D model that render it computationally efficient also impose restrictions on the time-scale of resolvable processes. Basically, they must be much longer than the vertical diffusion time scale.

Four progressively more complicated scenarios are investigated. The important factors governing the importance of bottom friction in each are found to be 1) non-dimensional surface Ekman depth, $u_{* s} / f h$ where $u_{* s}$ is the surface shear velocity, $f$ is the Coriolis parameter and $h$ is the water depth 2) the non-dimensional bottom roughness, $z_{0} / h$ where $z_{0}$ is the roughness length and 3) the angle between the wind stress and the shoreline. Each has significant influence on the drag law. The drag tensor magnitude, $r$, and the drag tensor angle, $\theta$ are functions of all three, while a drag tensor which scales with the square of the depth-averaged velocity has a magnitude, $C_{d}$, that only depends on $z_{0} / \mathrm{h}$.

The choice of drag law is found to significantly affect the response of a domain. Spin up times and phase relationships vary between models. In general, the $2 \frac{1}{2}$-D model responds more quickly than either a constant $r$ or constant $C_{d}$ model. Steady-state responses are also affected. The two most significant results are that failure to account for $\theta$ in the drag law sometimes leads to substantial errors in estimating the sea surface height and to extremely poor resolution of cross-shore bottom stress. The latter implies that cross-shore near-bottom transport is essentially neglected by traditional DAMs.
\end{abstract}

Thesis Supervisor: Ole Secher Madsen

Title: Professor, Civil Engineering 


\section{Acknowledgments}

I owe thanks to a lot of people for making my experience in the Joint Program an enjoyable one. In fact, there are too many to mention them all. Some, however, particularly stand out, and I would like to acknowledge them here.

My advisor for the last three years has been Ole Madsen. I've learned more from him than from any other person in my academic career. His physical intuition and ability to find the "essential" question are attributes that improved the quality of this thesis greatly and have, I hope, rubbed off a little on me. His friendship, enthusiasm and integrity have also been invaluable. They transformed what could have been a difficult situation into a very positive one.

My thesis committee, Bob Beardsley, Dave Chapman, Ken Melville and John Trowbridge deserve a great deal of thanks for assistance throughout the course of this work, including thorough and thoughtful reading of the dissertation. Hans Graber, who served as my defense chairman, rates special thanks for reading and commenting on the thesis and for providing much needed computer time.

Rocky Geyer provided access to his PC kingdom. Without this, preparation of the graphics and typesetting of the text for this thesis would have been extremely difficult. Rich Signell shared his computer expertise whenever I asked (thank goodness!).

Paul "Golf My Way" Dragos and Rich "Squigg" Signell provided me with enough distraction to stay sane and relaxed. If it's the last thing I do, I'm going to make a hole-in-one or a string of five three-pointers in a row!

My family has always provided a strong emotional foundation for me. My parents, Donald and Barbara Jenter; brother, Howard; and in-laws, Bob and Shirley Jones were constantly ready with a word of encouragement whenever necessary. To all of them, I owe a special thank you.

The biggest thanks of all goes to my wife, Deb, who shared the whole experience with me. It would have been a lot harder and a lot less fun without her. This thesis is dedicated to her.

Financial support during my time in graduate school came from the Woods Hole Oceanographic Institution and grants from the National Science Foundation (OCE84-03249) and the Office of Naval Research (N00014-86-K-0061). 


\section{Contents}

1 Introduction $\quad 11$

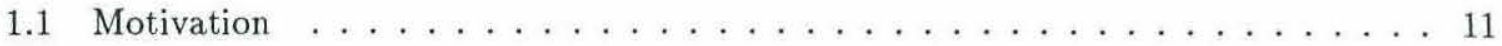

1.2 Problem Definition . . . . . . . . . . . . . . . . . . 13

1.3 "Traditional" Drag Laws . . . . . . . . . . . . . . . . . . . . 14

1.4 Alternatives to Traditional Drag Laws . . . . . . . . . . . . . . 15

1.5 The Present Approach . . . . . . . . . . . . . . . . 18

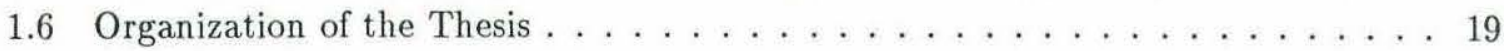

2 Model Formulation $\quad 20$

2.1 The Depth-averaged Model . . . . . . . . . . . . . . . 20

2.2 The Depth-Resolving Model . . . . . . . . . . . . . . . . . . 29

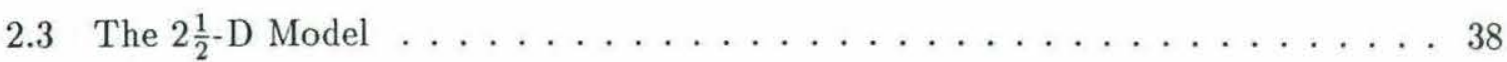

$3 \quad 2 \frac{1}{2}$-D Model Results $\quad 47$

3.1 Constant Depth Shelf, Straight Coastline . . . . . . . . . . . . . . . 49

3.1.1 Model domain and wind stress field . . . . . . . . . . . 49

3.1.2 Surface height and depth-averaged velocity $\ldots \ldots \ldots \ldots$

3.1.3 Momentum balances . . . . . . . . . . . . . . . . 61

3.1 .4 Bottom stress and drag tensor $\ldots \ldots \ldots \ldots 6 \ldots$

3.1.5 Differences between traditional and $2 \frac{1}{2}$-D model results $\ldots \ldots \ldots 77$

3.2 Linearly-Varying Depth, Straight Coastline . . . . . . . . . . . . . . 83

3.2 .1 Model domain and wind stress field . . . . . . . . . . . . . 83

3.2.2 Surface height and depth-averaged velocity . . . . . . . . . . . 84 
3.2 .3 Momentum balances . . . . . . . . . . . . . . . . . . 89

3.2 .4 Bottom stress and drag tensor . . . . . . . . . . . . 92

3.2.5 Differences between traditional and $2 \frac{1}{2}$-D model results . . . . . . . . 100

3.3 Linearly-Varying Depth, Sinusoidal Coastline . . . . . . . . . . . . . . . . 108

3.3.1 Model domain and wind stress field . . . . . . . . . . . . 108

3.3.2 Surface height and depth-averaged velocity . . . . . . . . . . 109

3.3.3 Bottom stress and drag tensor . . . . . . . . . . . . 118

3.3.4 Differences between traditional and $2 \frac{1}{2}$-D model results . . . . . . . . . 123

3.4 Linearly-Varying Depth, Straight Coastline, Rotating Wind Stress . . . . . . . 126

3.4 .1 Model domain and wind stress field . . . . . . . . . . . 126

3.4 .2 Sea surface height and depth-averaged velocity . . . . . . . . . 127

3.4 .3 Drag tensor . . . . . . . . . . . . . . . . . 133

3.4.4 Differences betweeen traditional and $2 \frac{1}{2}$-D model results . . . . . . . . . 139

4 Summary and Conclusions $\quad 141$

4.1 The Role of Bottom Stress . . . . . . . . . . . . . . . . . . . 141

4.2 Variation of the Drag Tensor . . . . . . . . . . . . . 143

4.3 Comparison of Drag Tensor and Traditional Drag Law Results . . . . . . . . 145

4.4 Conclusion and Recommendations for Future Work . . . . . . . . . . 146 


\section{List of Figures}

1-1 Schematic illustrating potential problems with "traditional" bottom stress for-

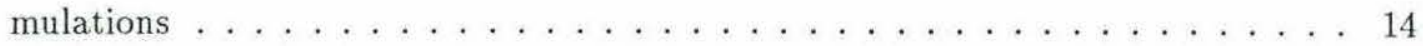

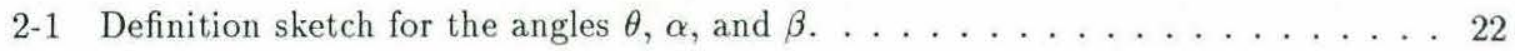

$2-2$ The Arakawa " $\mathrm{C}$ " grid . . . . . . . . . . . . . . . . . . 24

2-3 Variation of dimensionless linear drag tensor magnitude as a function of Ekman depth to water depth ratio . . . . . . . . . . . . 26

2-4 Variation of drag tensor direction as a function of Ekman depth to water depth

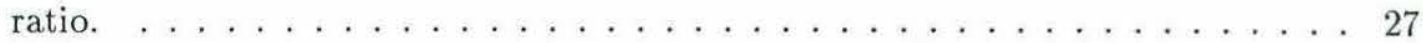

2-5 Variation of quadratic drag tensor magnitude as a function of Ekman depth to

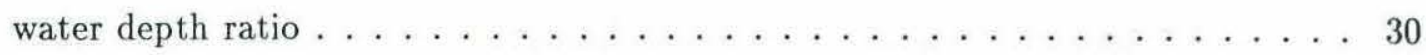

2-6 Schematic representation of the bilinear eddy viscosity. . . . . . . . . . . 32

2-7 Schematic of the depth-resolving model . . . . . . . . . . . . 39

$2-8$ Schematic of the full $2 \frac{1}{2}$-D model. . . . . . . . . . . . . 41

2-9 The Arakawa "C" grid showing superimposed $r, \theta$ nodes. . . . . . . . . . . . 42

3-1 Schematic representation of the four scenarios investigated . . . . . . . . 48

3-2 Time series of surface height at two different points across the shelf . . . . . . . 51

3-3 Contours of sea surface height in $y-t$ space for the shallow case $\ldots \ldots 53$

3-4 Contours of sea surface height in $y-t$ space for the intermediate case . . . . 54

3-5 Contours of sea surface height in $y-t$ space for the deep case . . . . . . . 55

3-6 3-D plot of sea surface height in $y-t$ space for the shallow case . . . . . . 56

3-7 3-D plot of sea surface height in $y-t$ space for the intermediate case . . . . 56

3-8 3-D plot of sea surface height in $y-t$ space for the deep case $\ldots \ldots \ldots 7$ 
3-9 Time series of cross-shore depth-averaged veloctiy at two points across the shelf . 59

3-10 Time series of alongshore depth-averaged veloctiy at two points across the shelf . 60

3-11 Time series of shallow case momentum balance components at the offshore point. 62

3-12 Time series of intermediate case momentum balance components at the offshore

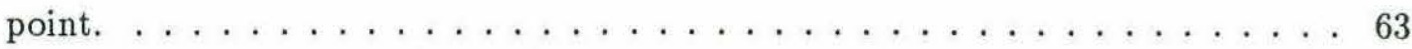

3-13 Time series of deep case momentum balance components at the offshore point. . 64

3-14 Time series of shallow case kinematic bottom stress components from the DAM

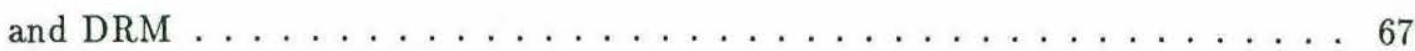

3-15 Time series of intermediate case kinematic bottom stress components from the

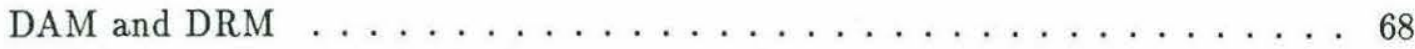

3-16 Time series of deep case kinematic bottom stress components from the DAM and

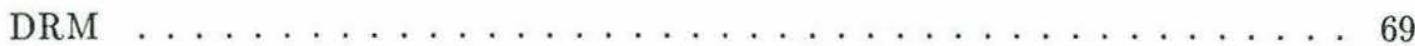

3-17 Time series of resistance coefficient at the offshore point. . . . . . . . . 71

3-18 Time series of estimated drag coefficient at the offshore point. . . . . . . . 72

3-19 Time series of shallow case angular information at the offshore point for early

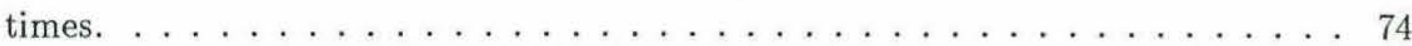

3-20 Time series of intermediate case angular information at the offshore point for

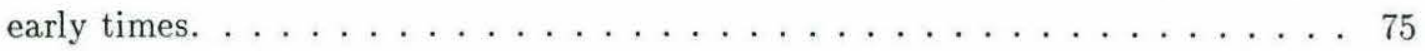

3-21 Time series of deep case angular information at the offshore point for early times. 76

3-22 Time series of sea surface height at the offshore point for different drag law assumptions: variable drag tensor, constant resistance coefficient and constant

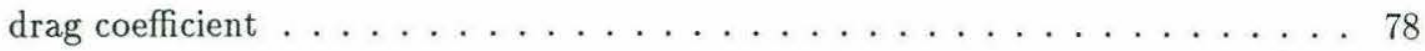

3-23 Time series of alongshore depth-averaged velocity at the offshore point for different drag law assumptions: variable drag tensor, constant resistance coefficient and constant drag coefficient . . . . . . . . . . . . 79

3-24 Time series of cross-shore depth-averaged velocity at the offshore point for different drag law assumptions: variable drag tensor, constant resistance coefficient

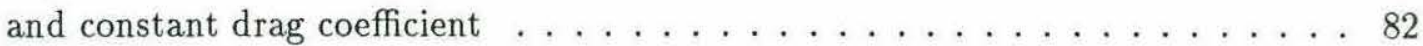

3-25 Time series of sea surface height, depth-averaged cross-shore velocity and depthaveraged alongshore velocity at two points on the shelf $\ldots \ldots \ldots$. . . . 86 
3-26 Contour plot of sea surface height on the $y$-t plane . . . . . . . . 87

3-27 3-D plot of sea surface height in $y$-t space . . . . . . . . . . . 88

3-28 Time series of momentum balance components at the onshore point. . . . . . . . 90

3-29 Time series of momentum balance components at the offshore point. . . . . . . . 91

3-30 Time series of resistance coefficient at the onshore and offshore points. . . . . . . 94

3-31 Time series of kinematic bottom stress magnitude at the offshore point . . . . . . 95

3-32 Time series of depth-averaged velocity magnitude at the offshore point . . . . . 9 96

3-33 Time series of estimated drag coefficient at the offshore and onshore points . . . 97

3-34 Time series of angular information at the onshore point. . . . . . . . . . . 98

3-35 Time series of angular information at the offshore point. . . . . . . . . . . . . 99

3-36 Steady-state surface profile for a wind angle of $0^{\circ} \ldots \ldots \ldots \ldots 1$

3-37 Steady-state surface profile for a wind angle of $30^{\circ} \ldots \ldots$. . . . . . . . . 102

3-38 Steady-state surface profile for a wind angle of $60^{\circ} \ldots \ldots \ldots \ldots$

3-39 Steady-state surface profile for a wind angle of $120^{\circ} \ldots \ldots \ldots$. . . . . . . 104

3-40 Steady-state surface profile for a wind angle of $150^{\circ} \ldots \ldots \ldots$

3-41 Steady-state surface profile for a wind angle of $180^{\circ} \ldots \ldots \ldots$

3-42 Contours of surface elevation at $2.4,4.8$ and $7.2 \mathrm{hrs} \ldots \ldots \ldots \ldots$

3-43 Contours plots of surface elevation at 9.6 and 12 hrs and steady-state . . . . 111

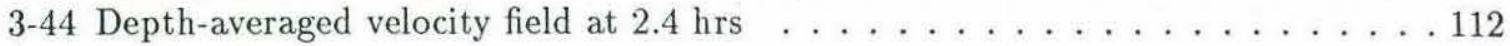

3-45 Depth-averaged velocity field at $4.8 \mathrm{hrs} \ldots \ldots \ldots \ldots \ldots \ldots$

3-46 Depth-averaged velocity field at $7.2 \mathrm{hrs} \ldots \ldots \ldots \ldots \ldots \ldots \ldots$

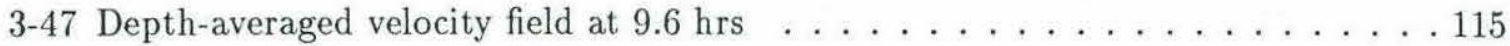

3-48 Depth-averaged velocity field at $12 \mathrm{hrs} \ldots \ldots \ldots \ldots \ldots \ldots \ldots$

3-49 Depth-averaged velocity field at steady-state . . . . . . . . . . . . . . 117

3-50 Contour plot of $\beta$ at steady-state . . . . . . . . . . . . . . . 119

3-51 Steady-state kinematic bottom stress field . . . . . . . . . . . . . . . 120

3-52 Steady-state resistance coefficient. . . . . . . . . . . . . . . . 121

3-53 Steady-state estimated drag coefficient. . . . . . . . . . . . . . . 121

3-54 Contour plot of $\alpha$ at steady-state . . . . . . . . . . . . . . 122

3-55 Contour plot of $\theta$ at steady-state . . . . . . . . . . . . . . . 123 
3-56 Contour plot of $\alpha$ (and, therefore, $\beta$ ) at steady-state from the traditional model . 124

3-57 Contour plot of the difference in steady-state $\beta$ fields between the $2 \frac{1}{2}$-D and traditional models . . . . . . . . . . . . . . . . 125

3-58 Contour plot of the difference in steady-state $\alpha$ fields between the $2 \frac{1}{2}-\mathrm{D}$ and traditional models . . . . . . . . . . . . . . . . 125

3-59 Time series of surface height, alongshore depth-averaged velocity and cross-shore depth-averaged velocity at the offshore and onshore points and time series of alongshore and cross-shore kinematic wind stress . . . . . . . . . 128

$3-60$ Contour plot of surface height on the $y$-t plane $\ldots \ldots \ldots \ldots$

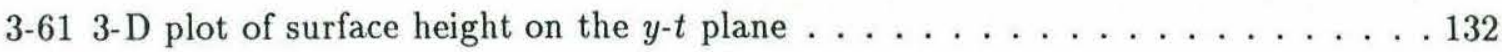

3-62 Time series of resistance coefficient at the offshore and onshore points . . . . 134

3-63 Time series of depth-averaged velocity magnitude and kinematic bottom stress at the offshore point and the onshore point . . . . . . . . 135

3-64 Time series of estimated drag coefficient at the offshore and onshore points . . 137

3-65 Time series of $\theta$ at the offshore and onshore points . . . . . . . . . 138

3-66 Time series of alongshore velocity for the $2 \frac{1}{2}-\mathrm{D}$ and the constant $r$ models at the onshore and offshore points . . . . . . . . . . . . . . 140 


\section{List of Tables}

2.1 Dimensionless surface Ekman depth, $u_{* s} / f h$, as a function of wind speed $10 \mathrm{~m}$ above the water surface, and water depth, $\ldots \ldots \ldots \ldots \ldots$

2.2 Range of dimensionless bottom roughness length, $z_{0} / h$, as a function of grain

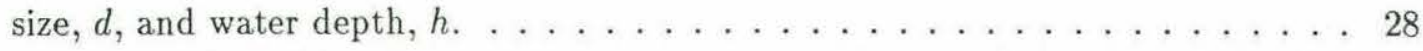

2.3 Comparison of speed and accuracy for analytical and numerical solutions with random forcings. ...................... 40

2.4 Fundamental seiching periods which might be interpreted as $t_{\text {pressure }}$ for $2 \frac{1}{2}$-D model runs . . . . . . . . . . . . . . . . . . . . 44

2.5 Diffusion times for various shear velocities in $20 m$ of water . . . . . . 45

3.1 Differences between traditional and $2 \frac{1}{2}$-D model steady-state results for the shallow case. . . . . . . . . . . . . . . . . . . 81 


\section{Chapter 1}

\section{Introduction}

This thesis presents a method for improving wind-driven depth-averaged circulation models (DAMs) by providing a more realistic estimate of bottom stress than traditional drag formulations. Improved DAMs should yield better predictions of coastal water levels and mass transports. In its existing form, the modelling approach used here is applicable to unstratified, wind-driven coastal flows. However, it can, with minimal effort, be adopted also to include tidally-driven flows.

Chapter 1 contains a brief motivating discussion based on the present state of wind-driven circulation modelling. In addition, a basic description of the modelling procedure and an outline of the thesis structure are given.

\subsection{Motivation}

The two most relevant problems to depth-averaged circulation modelling are storm surge and horizontal transport prediction. Each has obvious health and economic implications. Therefore, any method by which they can be improved seems worthwhile pursuing.

Storm surges are among the most devastating of natural disasters. Thousands of people have been killed and billions of dollars of property destroyed by storm surges in the United States alone (Murty, 1984). Similarly, Japan, India, Bangladesh and all of the European countries surrounding the North Sea have experienced catastrophic storm surges. In fact, few of the world's coastlines have been spared. 
Consequently, a great deal of interest in providing useful storm surge forecasting schemes has long existed in the oceanographic community. This is illustrated clearly by the fact that in 1957 enough literature on the subject had accumulated to prompt Charnock and Crease to write a review paper on storm surge observations and prediction. Since then, storm surge research has flourished (See additional reviews by Welander, 1961; Groen and Groves, 1962; Bretschneider, 1967; Heaps 1967, 1983; and a recent 900 page monograph by Murty, 1984).

Perhaps the biggest trend in storm surge forecasting over the last 30 years has been the shift from empirical to numerical prediction schemes. The move gained a great deal of momentum in the late 1960s with the introduction of a number of wind-driven coastal DAMs (e.g. Heaps, 1969). Three-dimensional (3-D) models soon followed and gained limited popularity in the mid1970s (e.g. Heaps and Jones, 1975). Both types have since developed significantly. Nonetheless, DAMs constitute the vast majority of operational storm surge forecasting models and are not likely to be replaced by $3-\mathrm{D}$ models in the near future. This is due primarily to the latter's extreme computational expense (Davies, 1989 personal communication).

Wind-driven transport modelling does not have nearly the long history that storm surge modelling does, nor has it enjoyed as much success. There are many reasons for this. Perhaps chief among them is the difficulty of making Lagrangian transport calculations due to their extreme sensitivity to small scale structure in the velocity field. Sea surface heights, on the other hand, are a more robust variable and, therefore, more easily predicted.

It has only been recently that widespread availability of supercomputers has led to improvements in small scale depth-averaged flow structure resolution (e.g. Signell and Geyer, 1989). In addition, the introduction of efficient coordinate systems (e.g. Blumberg and Herring, 1983) and other numerical tools has aided this effort.

Superimposed on the horizontal resolution problem is the difficulty that DAMs are often used to predict the transport of quantities that are not vertically well-mixed. This is usually attempted by introducing an artificial horizontal dispersion coefficient which is to account for vertical differences in transport (e.g. Kossik et al., 1987).

Despite their inherent problems, depth-averaged transport models stand to be improved in two ways by better estimates of bottom stress. The first is simply through improvement of the predicted flow structure. If the velocity field is modelled more accurately, the associated 
transport fields should be modelled more accurately too. The second is relevant to models where the transported quantity is known to be concentrated near the sea bottom. In such cases, more accurate predictions of bottom stress can be translated into better predictions of the advecting near-bottom flow field.

There is actually a third way that the method described here might be useful for transport modelling. However, it is not specifically addressed in this thesis. The method yields simplified estimates of the vertical profile of horizontal velocity at each grid point in a DAM. This information could be used to calculate better horizontal dispersion coefficients or to advect quantities differentially at different depths in an associated transport model or to obtain estimates of momentum transport coefficients for the DAM itself.

Clearly, the above discussion is not an exhaustive review of storm surge and depth-averaged transport modelling. Instead, it is intended to present the context in which improvements of bottom stress estimates may be useful. The comments above are not entirely speculation, however. In fact, Blumberg and Oey (1985) in a recent review cite lack of appropriate bottom stress formulations for DAMs as one of the most significant deficiencies in coastal circulation modelling at present.

\subsection{Problem Definition}

When the momentum and mass conservation equations governing wind-driven flow dynamics are integrated vertically, equations describing evolution of the sea surface elevation and depthaveraged velocity result. These equations contain terms representing both momentum sources and sinks. The sources are wind stress and the atmospheric pressure gradient. The sink is drag on the flow imposed by the seabed. Specification of the meteorological source terms is, in itself, a very difficult problem and is far from being satisfactorily resolved (Murty, 1984; Blumberg and Oey, 1985). Nonetheless, this thesis focuses only on the problem of specifying the bottom stress.

Because the bottom stress is a function of the flow, it must somehow be related to the dependent variables, depth-averaged velocity and sea surface height, and to the known meteorological forcing. This presents a severe problem as the bottom stress is a function of the near bottom flow field. Unfortunately, the bottom flow field is related to the dependent variables and 

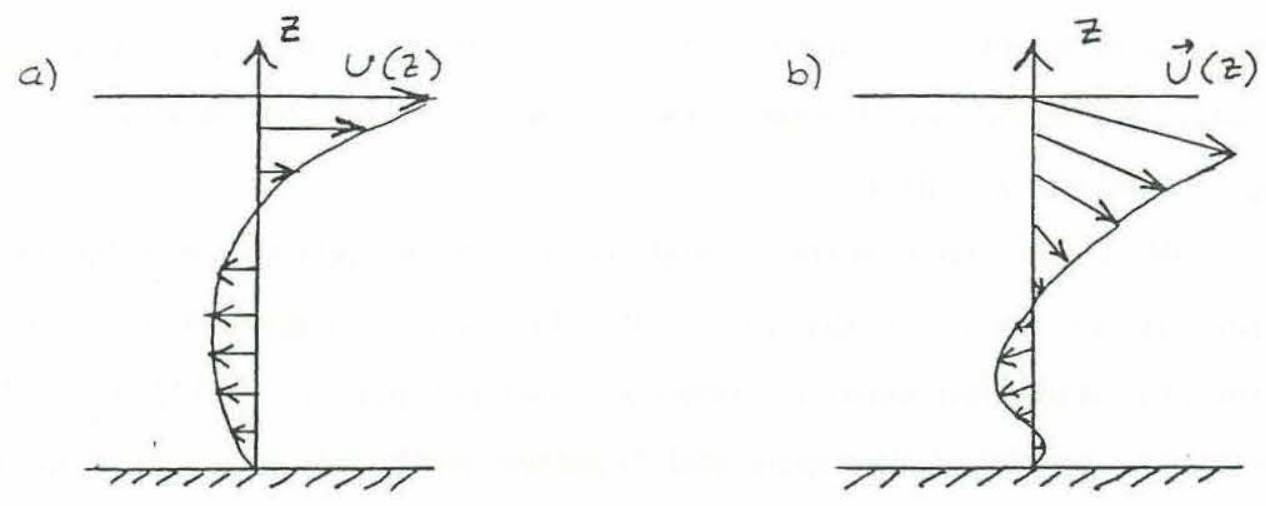

Figure 1-1: Schematic illustrating potential problems with "traditional" bottom stress formulations

forcing through a very complicated set of physics. The crux of the bottom stress formulation problem is the parameterization of the relationship between the bottom stress and the near bottom flow, dependent variable and forcing fields.

\section{3 "Traditional" Drag Laws}

The most common method of parameterizing bottom stress in DAMs is the quadratic drag law. In such a law, the bottom stress is equal to the product of the water density, the depth-averaged velocity vector, its magnitude, and a prescribed nondimensional drag coefficient. Quadratic drag laws are easy to implement and add little computational effort to the solution procedure. However, ease of use is potentially overshadowed by an oversimplification of physics.

A good example of problems caused by the physical oversimplification of quadratic drag laws is illustrated in figure 1-1a. The depth-averaged flow is equal to 0 but there is a finite flow near the bottom. The quadratic drag law predicts no bottom stress in this case even though it exists. A more important example of oversimplification is illustrated in figure 1-1b. Coriolis acceleration causes the flow to rotate with depth. Thus, the direction of the bottom stress is not that of the depth-averaged flow. The quadratic drag law predicts the wrong bottom stress by restricting it to be in the same direction as the depth-averaged velocity. 
Another traditional formulation used in place of the quadratic drag law is the linear drag law. In a linear drag law, bottom stress is formulated as the product of water density, depthaveraged velocity and an a priori specified resistance coefficient with dimensions [length/time]. The resistance coefficient can be thought of as a drag coefficient multiplied by a representative depth-averaged current speed since it essentially replaces the product of the drag coefficient and the variable depth-averaged velocity magnitude in the quadratic law. Linear drag laws retain the problems illustrated in figure 1-1.

Because the resistance coefficient is not temporally- or spatially-varying like the depthaveraged velocity magnitude, linear formulations are often considered more restrictive than quadratic drag laws. Consequently, they tend to be used more often in process-oriented models (e.g. Chapman, 1985) while quadratic laws are more frequently used in simulation models (e.g. Beardsley and Haidvogel, 1981).

This is not to say that linear drag laws are used only in process models. In fact, certain characteristics of linear drag laws make them "preferable" to quadratic drag laws. If the depthaveraged governing equations to be modelled are otherwise linear, using a linear drag law keeps them that way. This often improves the numerical stability of the associated model.

Unfortunately, linear drag laws are also chosen sometimes when a modeller simply assumes that bottom stress cannot be predicted accurately by either of the traditional drag laws. The computationally simpler linear drag law is chosen and the resistance coefficient is used as a tunable parameter to improve agreement between model results and observations.

\subsection{Alternatives to Traditional Drag Laws}

Various methods have been implemented to overcome the problems of traditional drag laws. Fixes range from adding a constant vector to the bottom stress to resolving the near bottom flow field with a 3-D model. Clearly, there is a trade-off between computational ease and realistic representation of bottom stress.

The difficulty with traditional drag laws illustrated in figure 1-1a was addressed by Bowden (1953) and Reid (1957). Both examined the situation of steady flow forced by a wind perpendicular to a vertical wall. In the depth-averaged sense, there is no flow perpendicular to the wall. Therefore, traditional drag laws predict no bottom stress in that direction. However, in 
reality, a strong wind blowing directly perpendicular to a wall would force a surface flow toward the wall which would be balanced by a return flow near the bottom.

Bowden used a constant eddy viscosity model to resolve the flow field and to show that the bottom stress (force on the bottom by the flow) for laminar flow should be between $0 \%$ and $50 \%$ of the surface stress and directed upwind. Reid used a more complicated mixing length model to suggest that for turbulent flow the bottom stress should be around $10 \%$ of the surface stress and directed upwind. These ideas were generalized by others (e.g. Groen and Groves, 1962) who suggested that traditional drag laws should be modified by adding a constant fraction (typically 10\%, after Reid) of the wind stress to the bottom stress. This idea breaks down immediately, however, for a strong wind stress parallel to a wall (or a straight coastline). In such a case, the flow is entirely downwind throughout the water column and the stress exerted by the flow on the seabed is downwind rather than upwind.

Jenter and Madsen (1989) used a steady-state bi-linear eddy viscosity model (a modified version of which is described in Chapter 2) to examine the idea of adding a constant fraction of the wind stress to the bottom stress. They found that the ratio of cross-shore bottom stress to cross-shore wind stress was a complicated function of bottom roughness, wind strength and angle between the wind and the wall. Using a different eddy viscosity, Davies (1987) also found a large amount of variability in the fractional relationship between cross-shore bottom stress and surface stress. Jamart and Ozer (1987) analytically examined steady-state wind-driven flow in a closed basin and showed that the relationship between bottom stress and surface stress necessarily included other terms beside the traditional drag term and a fraction of the wind stress. There is ample evidence that using a predetermined constant fraction of the wind stress to improve bottom stress in DAMs is inappropriate.

A slightly more involved approach than the drag laws described above is used in so-called $2 \frac{1}{2}$-dimensional ( $2 \frac{1}{2}$-D) models where bottom stress is predicted for a DAM by estimating the bottom flow structure with a separate one-dimensional (1-D) model. The upper complexity limit for $2 \frac{1}{2}$-D models might be considered a fully 3-D model. However, as used in this thesis, $2 \frac{1}{2}$-D model refers to a DAM coupled to a simplified depth-resolving model (DRM). Therefore, the term implies that the combined model improves the bottom stress estimates by resolving the near bottom flow structure while not entirely sacrificing the computational efficiency of a 
DAM.

Many $2 \frac{1}{2}$-D models have their origin in the work of Welander (1957), who showed that for a constant eddy viscosity, the bottom stress can be related to time histories of the wind stress and sea surface slope through a convolution integral. Since the wind stress is known and the surface slope at any point can be calculated from the depth-averaged equations, the bottom stress integral can be evaluated in parallel with the DAM yielding improved bottom stress estimates. Jelesnianski (1970) provided a method of analytically simplifying the convolution integral and applied it in a storm surge model. Forristall (1974) developed a similar convolution integral and applied it in a study of storm-generated currents. Kielmann and Kowalik (1980) used a combination of finite difference and analytic methods to approximate the convolution integral.

The convolution integral models necessarily employ a constant eddy viscosity. A more flexible structure is obviously desirable. To this end, Nihoul (1977) introduced a technique by which a temporally and vertically-variable eddy viscosity can be used, provided the eddy viscosity is separable in depth and time. Separability allowed Nihoul to specify the velocity profile as the sum of a set of vertical modes with time-dependent amplitudes but time-invariant vertical structure. The shape functions for the modes are chosen to be eigenfunctions of a second-order ordinary differential equation involving the depth-dependent part of the eddy viscosity. Much like evaluating the convolution integrals, calculating the mode amplitudes corresponds to computing an inverse Laplace transform involving the sea surface slope and boundary shear stresses. Resultant expressions for the near bottom flow can be inverted numerically with some difficulty to relate the bottom stress to the depth-averaged velocity, sea surface slope and wind-stress.

Independently, Jordan and Baker (1980) and Baker and Jordan (1980,1981) developed similar expansion techniques. Davies (1987) applied the method and showed that, with the appropriate problem formulation, mode amplitudes could be calculated by recursion relationships greatly simplifying determination of bottom stress for the DAM. Davies also claimed that only 2 to 4 modes were necessary to yield DAM results comparable to those of a fully 3-D model. Unfortunately, his test case was in water so deep that bottom stress was probably not dynamically important. Because of this, major differences in bottom stress formulations might not produce noticeable differences in model output, rendering his results inconclusive.

The convolution and eigenfunction methods are a clear improvement over traditional drag 
laws, but have two potential problems. The first, and most obvious, is that the form of eddy viscosity is restricted to be, at best, separable in depth and time and, at worst, constant. Complicated flow regimes in shallow wind-driven waters necessitate violation of these restrictions. The presence of both surface and bottom boundary layers, which interact and vary in relative strength, requires the eddy viscosity to adjust its relative strength near the surface and bottom boundaries as a function of time, which cannot happen in the convolution and eigenfunction formulations.

The second problem is more subtle. The convolution and eigenfunction methods require that either a slip or no-slip boundary condition be applied at the sea bed. A slip condition relates near bottom velocity to near bottom shear, and a no slip condition requires the near bottom velocity to vanish. Each can impose unrealistic solutions on the near bottom flow profile when applied individually. More realistically, the bottom flow should satisfy both no-slip and shear conditions. In order to do this, however, the eddy viscosity must be a function of the flow. Such a relationship precludes a priori knowledge of the eddy viscosity function.

Unfortunately, the problem of allowing eddy viscosity to be flow-dependent is perhaps best addressed by second-order closure models (e.g. Mellor and Yamada, 1974) in which timeand depth-dependent differential equations for turbulent velocity and length scales are solved. Models have been formulated with second-order closure schemes (e.g. Blumberg and Mellor, 1987 and Johns and Oguz, 1987), but at a substantial computational cost. Noyes and Stevens (1987), in fact, claim that the cost of including length and velocity scale equations increases 3-D model computational and storage requirements by a minimum of $30 \%$. Scholtz et al. (1987) have formulated a model with second-order closure that might be construed as an extreme 2 $\frac{1}{2}$-D model because the differential equations for the length and velocity scales are replaced by algebraic approximations. Nonetheless, the required computational effort must be at least comparable to a 3-D model without second-order closure since the momentum equations are not simplified.

\subsection{The Present Approach}

The goal of the work reported in this thesis is to produce a $2 \frac{1}{2}$-D formulation that incorporates realistic yet simple turbulent closure assumptions. The DRM employs an eddy viscosity closure 
that yields quasi-analytical velocity profiles satisfying the $2 \frac{1}{2}$-D model criterion for computational efficiency. Despite permitting an analytical solution, the eddy viscosity is quite realistic. It has a time-dependent functional form which strongly relates it to the near bottom flow field. Consequently, it can satisfy both shear and no-slip bottom boundary conditions. Also, because the problems of interest are wind-driven, the eddy viscosity accounts for the presence of a surface boundary layer and for the possibility that it will interact with the bottom boundary layer in shallow enough water.

The DRM communicates bottom stress information to the DAM by estimating a drag tensor at each DAM grid point. The tensor is used in the DAM to scale and rotate the depth-averaged velocity to yield an improved estimate of bottom stress. This eliminates the problem illustrated in figure 1-1b because the depth-averaged velocity and bottom stress do not have to be in the same direction. However, the problem described in 1-1a is not eliminated since there is still no bottom stress when the depth-averaged velocity is exactly equal to 0 .

The drag tensor formulation is partially chosen for reasons of computational efficiency. With it, the bottom stress need not be updated at every time step of the DAM. Bottom stress can still vary between updates because it is also a function of the depth-averaged velocity.

\subsection{Organization of the Thesis}

Modelling details are described in Chapter 2 including discussion of the DAM, the DRM and the combined $2 \frac{1}{2}$-D model. Chapter 3 contains $2 \frac{1}{2}$-D model results for a variety of wind-driven coastal flow situations. Chapter 4 is a summary of these results and their implications for wind-driven depth-averaged flow modelling. 


\section{Chapter 2}

\section{Model Formulation}

\subsection{The Depth-averaged Model}

Since the goal of this thesis is to provide an improved method of estimating bottom stress in wind-driven DAMs, a standard DAM was chosen as a starting point. The model was developed by Beardsley and Haidvogel (1981) to model the storm response of the Mid-Atlantic Bight. It has since been employed by Chapman (1985) to test a variety of open boundary conditions. Well documented model application was a primary factor in its selection here.

The governing equations are the vertically-integrated, linearized momentum and continuity equations for an unstratified fluid. They are

$$
\begin{aligned}
\frac{\partial U}{\partial t}-f V & =-g h \frac{\partial \zeta}{\partial x}+\frac{\tau_{s x}}{\rho}-\frac{\tau_{b x}}{\rho} \\
\frac{\partial V}{\partial t}+f U & =-g h \frac{\partial \zeta}{\partial y}+\frac{\tau_{s y}}{\rho}-\frac{\tau_{b y}}{\rho} \\
\frac{\partial \zeta}{\partial t} & =-\frac{\partial U}{\partial x}-\frac{\partial V}{\partial y}
\end{aligned}
$$

where $\zeta$ is sea surface displacement, $U$ alongshelf $(x)$ transport $\left([U]=\right.$ length ${ }^{2} /$ time $), V$ crossshelf $(y)$ transport, $g$ gravitational acceleration, $h$ water depth, $f$ the Coriolis parameter, $\rho$ water density, and $\left(\tau_{b x}, \tau_{b y}\right)$ and $\left(\tau_{s x}, \tau_{s y}\right)$ are the $(x, y)$ bottom and surface stresses, respectively.

Numerical considerations notwithstanding, and presuming the wind stress is known, solution of the system (2.1), (2.2) and (2.3) depends entirely on correct specification of the a priori unknown bottom stress. As discussed in Chapter 1, this is usually done by explicitly relating 
the bottom stress to the transport through a drag law. In a recent review of numerical coastal circulation modeling, Blumberg and Oey (1985) consider the specification of appropriate drag laws to be one of the single biggest problems facing depth-averaged modellers at present.

The two most common bottom stress formulations in DAMs are drag coefficients and resistance coefficients. In a drag coefficient formulation, bottom stress is related to transport through the quadratic expression

$$
\vec{\tau}_{b}=\rho C_{d} \frac{|\vec{U}| \vec{U}}{h^{2}}
$$

where the drag coefficient, $C_{d}$, is dimensionless. In a resistance coefficient formulation, bottom stress is related to transport through the linear expression

$$
\vec{\tau}_{b}=\rho \frac{r}{h} \vec{U}
$$

where the resistance coefficient, $r$, has dimensions [length/time]. Both (2.4) and (2.5) imply the very restrictive assumption that bottom stress and transport are in the same direction. However, due to the possible importance of Coriolis accelerations in the momentum balance, the two are not likely to be colinear (i.e. $\vec{\tau}_{b} \times \vec{U} \neq 0$ ) because bottom stress is determined by the near-bottom flow structure, and not an average over the water column.

Adopting the basic idea that bottom stress and transport need not be colinear, the first improvement suggested in this thesis is the introduction of a drag tensor. With a drag tensor, both scaling and rotation of the transport vector are performed in order to produce a bottom stress vector. For the drag coefficient, this is written as

$$
\left[\begin{array}{c}
\tau_{b x} \\
\tau_{b y}
\end{array}\right]=\rho \frac{\left(U^{2}+V^{2}\right)^{1 / 2}}{h^{2}}\left[\begin{array}{ll}
C_{d, x x} & C_{d, x y} \\
C_{d, y x} & C_{d, y y}
\end{array}\right]\left[\begin{array}{l}
U \\
V
\end{array}\right]
$$

and, for the resistance coefficient, as

$$
\left[\begin{array}{c}
\tau_{b x} \\
\tau_{b y}
\end{array}\right]=\frac{\rho}{h}\left[\begin{array}{ll}
r_{x x} & r_{x y} \\
r_{y x} & r_{y y}
\end{array}\right]\left[\begin{array}{l}
U \\
V
\end{array}\right]
$$

To emphasize the scaling and rotational nature of (2.6) and (2.7), they can also be written as

$$
\left[\begin{array}{c}
\tau_{b x} \\
\tau_{b y}
\end{array}\right]=\rho C_{d} \frac{\left(U^{2}+V^{2}\right)^{1 / 2}}{h^{2}}\left[\begin{array}{rr}
\cos \theta & -\sin \theta \\
\sin \theta & \cos \theta
\end{array}\right]\left[\begin{array}{l}
U \\
V
\end{array}\right]
$$




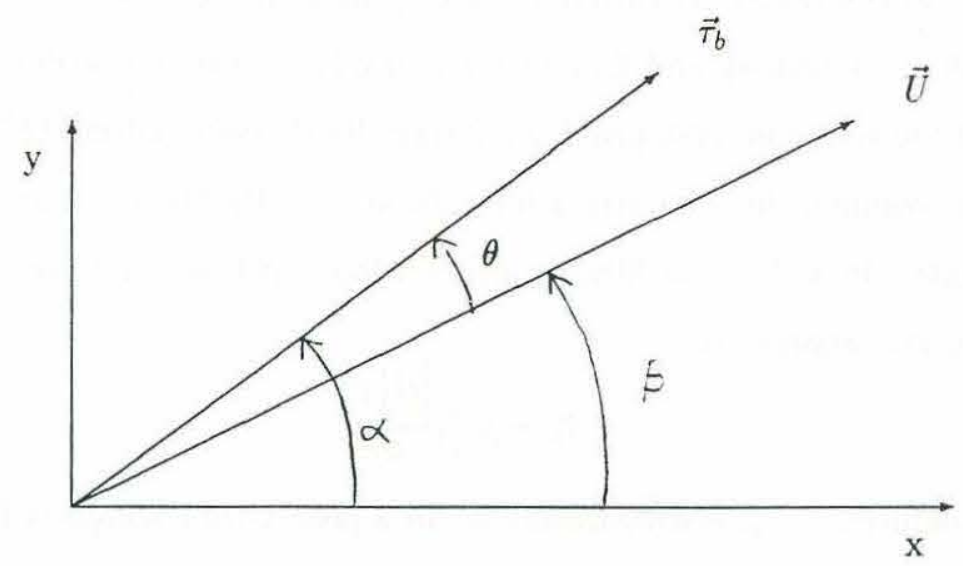

Figure 2-1: Definition sketch for the angular difference, $\theta$, between bottom stress, $\vec{\tau}_{b}$ and transport, $\vec{U}$. Also the angle between the bottom stress and $x$-axis, $\alpha$, and between the transport and $x$-axis, $\beta$ are shown.

and

$$
\left[\begin{array}{c}
\tau_{b x} \\
\tau_{b y}
\end{array}\right]=\rho \frac{r}{h}\left[\begin{array}{rr}
\cos \theta & -\sin \theta \\
\sin \theta & \cos \theta
\end{array}\right]\left[\begin{array}{l}
U \\
V
\end{array}\right]
$$

respectively.

Taking the vector norm of (2.8) and (2.9) and rearranging the results yields

$$
C_{d} \frac{|\vec{U}|^{2}}{h^{2}}=\frac{\left|\vec{\tau}_{b}\right|}{\rho}
$$

and

$$
r \frac{|\vec{U}|}{h}=\frac{\left|\vec{\tau}_{b}\right|}{\rho}
$$

which imply that $C_{d}$ and $r$ play the same role in (2.8) and (2.9) as they do in traditional drag laws. In other words, they are still the factors by which the magnitude of the depth-averaged velocity is scaled to yield the magnitude of the bottom stress.

The drag tensor angle, $\theta$, also has physical significance and an analog in traditional drag laws. It is the angle between the transport and bottom stress (measured counter-clockwise from the transport, as shown in figure 2-1). Traditional drag laws simply assume $\theta=0$. Notice that choosing $\theta=0$ reduces (2.8) and (2.9) to (2.4) and (2.5).

The amount of improvement offered by a drag tensor formulation lies in the extent to which 
$C_{d}$ or $r$ and $\theta$ can be correctly specified. A primary goal of this thesis is to find a computationally simple way of accurately specifying drag tensors in typical wind-driven coastal environments.

Introducing (2.9) into (2.1) and (2.2) yields modified depth-integrated momentum equations ${ }^{1}$

$$
\begin{aligned}
& \frac{\partial U}{\partial t}-f V=-g h \frac{\partial \zeta}{\partial x}+\frac{\tau_{s x}}{\rho}-\frac{r}{h} U \cos \theta+\frac{r}{h} V \sin \theta \\
& \frac{\partial V}{\partial t}+f U=-g h \frac{\partial \zeta}{\partial y}+\frac{\tau_{s y}}{\rho}-\frac{r}{h} U \sin \theta-\frac{r}{h} V \cos \theta
\end{aligned}
$$

It is interesting to note that (2.12) and (2.13) can also be written as

$$
\begin{aligned}
& \frac{\partial U}{\partial t}-\tilde{f} V=-g h \frac{\partial \zeta}{\partial x}+\frac{\tau_{s x}}{\rho}-\frac{\tilde{r}}{h} U \\
& \frac{\partial V}{\partial t}+\tilde{f} U=-g h \frac{\partial \zeta}{\partial y}+\frac{\tau_{s y}}{\rho}-\frac{\tilde{r}}{h} V
\end{aligned}
$$

where

$$
\tilde{f}=f+\frac{r}{h} \sin \theta
$$

and

$$
\tilde{r}=r \cos \theta
$$

In other words, the transport equations with a drag tensor can be arranged in such a way that they appear identical to the transport equations with a colinear resistance law. This means that the drag tensor (2.9) merely appears as a redefinition of the Coriolis parameter and resistance coefficient. Consequently, the numerical scheme employed by Chapman (1985) need not be changed at all to accomodate a drag tensor, so long as the new definitions are accepted.

The finite difference equations corresponding to (2.14), (2.15) and (2.3) are

$$
\begin{aligned}
\frac{U_{i, j}^{n+1}-U_{i, j}^{n}}{\Delta t}-\overline{f V}_{i, j}^{n} & =-g h \frac{\zeta_{i, j}^{n}-\zeta_{i-1, j}^{n}}{\Delta x}+\frac{\tau_{s x}^{n+1}}{\rho}-\frac{\tilde{r}}{h} U_{i, j}^{n+1} \\
\frac{V_{i, j}^{n+1}-V_{i, j}^{n}}{\Delta t}+\tilde{f} \bar{U}_{i, j}^{n+1} & =-g h \frac{\zeta_{i, j}^{n}-\zeta_{i, j-1}^{n}}{\Delta x}+\frac{\tau_{s y}^{n+1}}{\rho}-\frac{\tilde{r}}{h} V_{i, j}^{n+1} \\
\frac{\zeta_{i, j}^{n+1}-\zeta_{i, j}^{n}}{\Delta t} & =-\frac{U_{i+1, j}^{n+1}-U_{i, j}^{n+1}}{\Delta x}-\frac{V_{i, j+1}^{n+1}-V_{i, j}^{n+1}}{\Delta x}
\end{aligned}
$$

\footnotetext{
${ }^{1}$ For brevity, parallel derivations of equations using (2.8) are no longer carried out. Instead the linear formulation is exclusively employed. Similar derivations for the quadratic formulation are straightforward. However, since $r$ is a time-varying quantity in the $2 \frac{1}{2}$-D model, the choice of a linear instead of quadratic drag tensor is insignificant.
} 


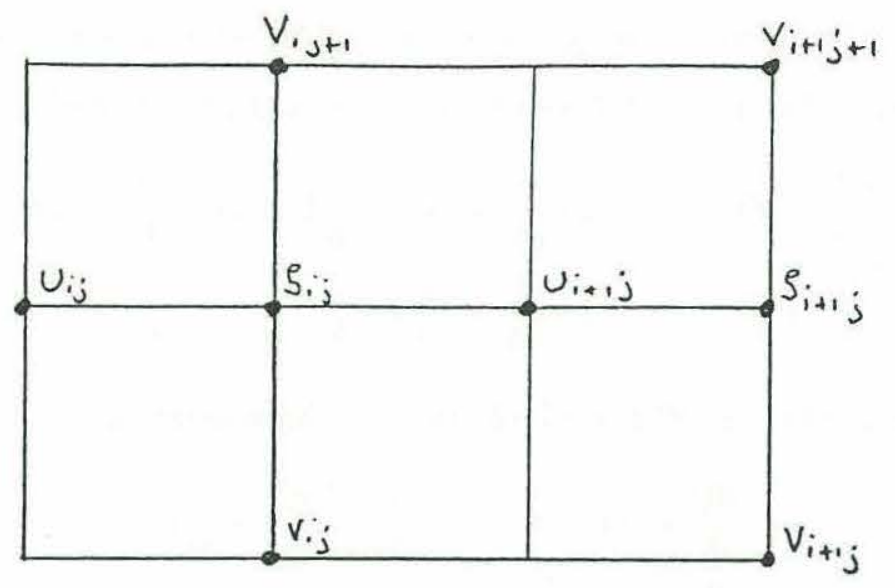

Figure 2-2: The Arakawa "C" grid

The superscript $n$ implies that the variable is evaluated at time, $t=n \Delta t$ where $\Delta t$ is the model time step. The subscripts $i$ and $j$ imply that the variable is evaluated at the $i^{\text {th }}$ element in the $x$-direction and the $j^{\text {th }}$ element in the $y$-direction of the grid. The grid spacing in both the $x$ and $y$ directions is assumed constant and equal to $\Delta x$. An overbar indicates that an average of the variable over adjacent grid elements is required (see Beardsley and Haidvogel 1981 for details.).

The grid used for the DAM is an Arakawa "C" grid shown in figure 2-2. The "C" grid is a spatially staggered grid chosen for its gravity wave propagation characteristics, its ability to handle coastal boundary conditions simply and the convenience with which centered spatial differences are calculated. Grid characteristics are thoroughly discussed by Mesinger and Arakawa (1976).

One difficulty encountered with the staggered grid is its inability to handle the Coriolis terms simply in the transport equations because $U$ and $V$ are not defined at the same nodes. Thus, a spatial averaging of transports for the Coriolis terms is required as indicated in (2.18) and (2.19). The DAM uses an averaging scheme developed by Platzman (1972) where surrounding transports are first weighted by inverse depth then averaged. The complete expression is given in the original model description by Beardsley and Haidvogel (1981).

Time differencing is done explicitly by a forward/backward scheme. In such a scheme, 
transports are stepped forward alternately with surface elevation as if staggered in time by $\Delta t / 2$. Von Neumann stability analysis of the forward/backward scheme for gravity wave propagation yields a Courant-Friedrichs-Lewy (CFL) stability criterion (Mesinger and Arakawa, 1976)

$$
\Delta t \leq \frac{1}{\sqrt{2}} \frac{\Delta x}{\sqrt{g h}}
$$

Note that the on-axis friction terms, $\tilde{r} \vec{U} / h$, are formulated implicitly with respect to the transport. Partial justification for doing so comes from Von Neumann stability analysis of (2.18), (2.19) and (2.20) with $\tilde{f}=0, \vec{\tau}_{s}=0$ and $\tilde{r}=$ constant, which shows that an explicit form yields a stability criterion $\Delta t<2 h / \tilde{r}$ in addition to (2.21) (Chapman, 1987 personal communication). With implicit friction terms, however, the numerical scheme is unconditionally stable with respect to constant $\tilde{r}$. The implicit scheme is adopted here with the hope that the stability characteristics for constant $\tilde{r}$ are inherited by the $2 \frac{1}{2}$-D model with variable $\tilde{r}$.

So far, nothing has been said about actual values of the drag tensor. Jenter and Madsen (1989) used a DRM identical to the one to be described in section 2.2 in order to compute values of $r$ and $\theta$ for the simple case of steady wind-driven flow in an ocean of infinite horizontal extent (i.e. no pressure gradients). Figures 2-3 and 2-4 depict drag tensor variation as a function of dimensionless surface Ekman depth, $u_{* s} / f h$, where $u_{* s}$ is the surface shear velocity defined $u_{* s}=\sqrt{\left|\vec{\tau}_{s}\right| / \rho}$. The different curves in each figure are for different ratios of bottom roughness length to water depth, $z_{0} / h$.

In order to facilitate interpretation of figures $2-3$ and 2-4, Table 2.1 contains values of $u_{* s} / f h$ for different wind speeds and water depths. The wind speeds are converted into stresses by application of Wu's (1982) formula and $f$ is taken to be $10^{-4} \mathrm{~s}^{-1}$, as it is throughout this thesis. The cases in the upper righthand half of the table (i.e. above the thick line) are of little interest or dynamical importance for storm surge and transport modelling. Therefore, this thesis concentrates on cases taken from the remainder of Table 2.1.

Table 2.2 contains typical values of $z_{0} / h$ for different sediment types. The roughness lengths are based on Nikuradse sand grain roughnesses, $z_{0}=d / 30$ where $d$ is the grain diameter. It may seem strange that pebbles and cobbles are included in the table, since they are not typical bottom types for coastal areas. Grant and Madsen $(1979,1982)$, showed, however, that the presence of surface gravity waves or bed forms can cause the flow to "feel" an enhanced 


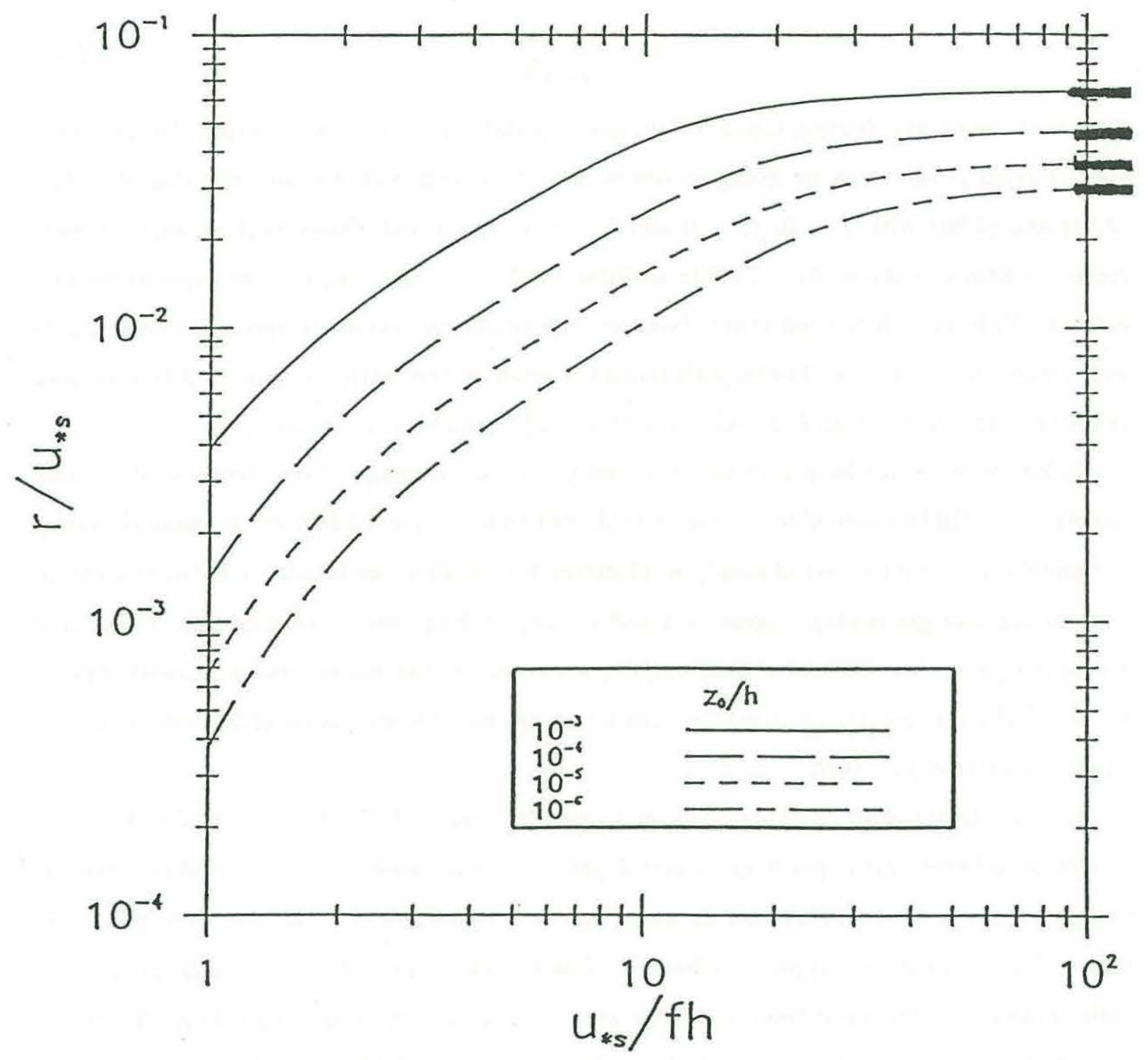

Figure 2-3: Variation of dimensionless linear drag tensor magnitude as a function of Ekman depth to water depth ratio (from Jenter and Madsen, 1989). 


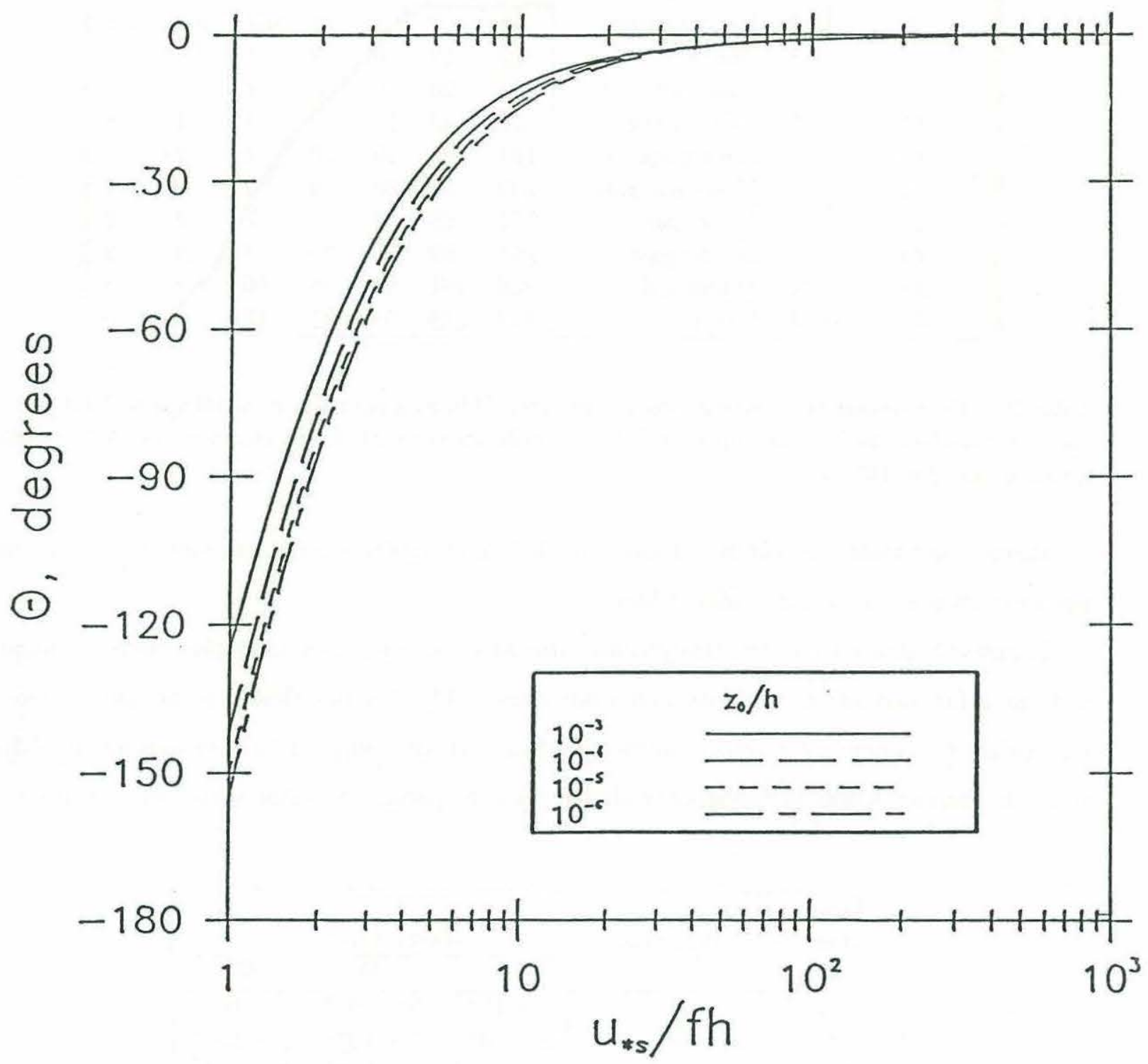

Figure 2-4: Variation of drag tensor direction as a function of Ekman depth to water depth ratio (from Jenter and Madsen, 1989). 


\begin{tabular}{|c|c|c|c|c|c|c|c|c|c|}
\hline \multirow{2}{*}{$\begin{array}{c}\text { Wind speed } \\
\text { at } 10 \mathrm{~m}, \\
\mathrm{~m} / \mathrm{s}\end{array}$} & \multirow{2}{*}{\multicolumn{2}{|c|}{$\begin{array}{c}\text { Beaufort } \\
\text { number, } \\
\text { description }\end{array}$}} & \multicolumn{7}{|c|}{ Water depth, $m$} \\
\hline & & & 2 & 5 & 10 & 20 & 50 & 100 & 200 \\
\hline 1 & 1 & Light air & 5 & 2 & 1 & 0.5 & 0.2 & 0.1 & 0.1 \\
\hline 3 & 2 & Light breeze & 16 & 7 & & 2 & 0.7 & 0.3 & 0.2 \\
\hline 5 & 3 & Gentle breeze & 29 & 12 & & 3 & 1 & 0.6 & 0.3 \\
\hline 8 & 4 & Moderate breeze & 51 & 20 & 10 & & 2 & 1 & 0.5 \\
\hline 11 & 5 & Fresh breeze & 75 & 30 & 15 & & 3 & 1 & 0.8 \\
\hline 14 & 6 & Strong breeze & 101 & 41 & 20 & 10 & & 2 & 1 \\
\hline 17 & 7 & Moderate gale & 130 & 52 & 26 & 13 & & 3 & 1 \\
\hline 21 & 8 & Fresh gale & 171 & 68 & 34 & 17 & & 3 & 2 \\
\hline 24 & 9 & Strong gale & 204 & 82 & 41 & 20 & 8 & 4 & 2 \\
\hline 28 & 10 & Whole gale & 250 & 100 & 50 & 25 & 10 & & 3 \\
\hline 33 & 11 & Storm & 313 & 125 & 63 & 31 & 13 & & 3 \\
\hline
\end{tabular}

Table 2.1: Dimensionless surface Ekman depth, $u_{* s} / f h$, as a function of wind speed $10 \mathrm{~m}$ above the water surface, and water depth, $h$. Wind speeds are converted to stresses using Wu's (1982) formula and $f=10^{-4} \mathrm{~s}^{-1}$.

roughness comparable to cobbles. Therefore, the larger values of $z_{0} / h$ are still relevant to the wind-driven coastal flows considered here.

Figure 2-3 shows that the steady-state value of $r$ can vary over multiple orders of magnitude as a function of water depth and wind stress. This implies that a priori knowledge of $r$ is highly dependent on a priori knowledge of $u_{* s}$. It also suggests that situations involving spatially variable winds or topography should require spatially variable values of $r$ in order to

\begin{tabular}{||c|l|ccc||}
\hline \multirow{2}{*}{$\begin{array}{c}\text { Grain } \\
\text { diameter, } \\
c m\end{array}$} & \multirow{2}{*}{ Size class } & \multicolumn{3}{|c}{ Water depth, m } \\
\cline { 3 - 5 } & & 2 & 20 & 200 \\
\hline 10. & Cobbles & $2 \times 10^{-3}$ & $2 \times 10^{-4}$ & $2 \times 10^{-5}$ \\
1. & Pebbles & $2 \times 10^{-4}$ & $2 \times 10^{-5}$ & $2 \times 10^{-6}$ \\
0.1 & Coarse Sand & $2 \times 10^{-5}$ & $2 \times 10^{-6}$ & $2 \times 10^{-7}$ \\
0.05 & Medium Sand & $8 \times 10^{-6}$ & $8 \times 10^{-7}$ & $8 \times 10^{-8}$ \\
0.025 & Fine Sand & $4 \times 10^{-6}$ & $4 \times 10^{-7}$ & $4 \times 10^{-8}$ \\
0.001 & Silt & $2 \times 10^{-7}$ & $2 \times 10^{-8}$ & $2 \times 10^{-9}$ \\
\hline
\end{tabular}

Table 2.2: Range of dimensionless bottom roughness length, $z_{0} / h$, as a function of grain size, $d$, and water depth, $h$. 
model bottom stress correctly. Similarly, situations with temporally variable winds will require temporally variable values of $r$.

Like figure 2-3, figure 2-4 implies a great deal of drag tensor variation with wind speed and water depth. It is apparent that, for a steady-state infinite horizontal extent ocean, a colinear drag law (i.e. $\theta=0$ ) applies only in extremely shallow water or for extremely high winds. As with $r$, it appears that a priori knowledge of $\theta$ is highly dependent on a priori knowledge of $u_{* s}$. Consequently, any spatial or temporal variation in the wind or water depth should require variation in $\theta$.

At least for an ocean of infinite horizontal extent, spatial and temporal variation of $\theta$ is of more concern than variation in $r$ because much of the variability in $r$ can be removed by assuming a quadratic drag law. Figure 2-5 (also from Jenter and Madsen, 1989) shows variation of $C_{d}$ as a function of Ekman depth to water depth ratio. Clearly, $C_{d}$ is not nearly as sensitive as $r$ to $u_{* s} / f h$. In fact, variation in $C_{d}$ over the important range of $u_{* s} / f h>5$ is comparable to that associated with a factor of 2 uncertainty in the bottom roughness.

Having established that the drag tensor is sensitive to forcing and topographic variation, a method by which $r$ and $\theta$ can be systematically varied in space and time is introduced. A DRM is used to update periodically the drag tensor at each grid element of the DAM. The combination of the DAM and DRM constitutes the $2 \frac{1}{2}$-D model.

Notice that adoption of the $2 \frac{1}{2}$-D modelling strategy means that the choice to use $r$ instead of $C_{d}$ is now immaterial because the drag tensor is allowed to vary in time and space. Therefore, provided it is updated often enough, temporal changes in forcing should produce appropriate changes in the drag tensor.

\subsection{The Depth-Resolving Model}

This section describes a quasi-analytical depth-resolving model, DRM, used for predicting vertical profiles of horizontal velocity in a wind- and pressure-driven unstratified fluid. The resulting profiles are, in turn, used to determine a drag tensor for the DAM.

Derivation of the DRM is based on a set of linearized momentum equations that can be 


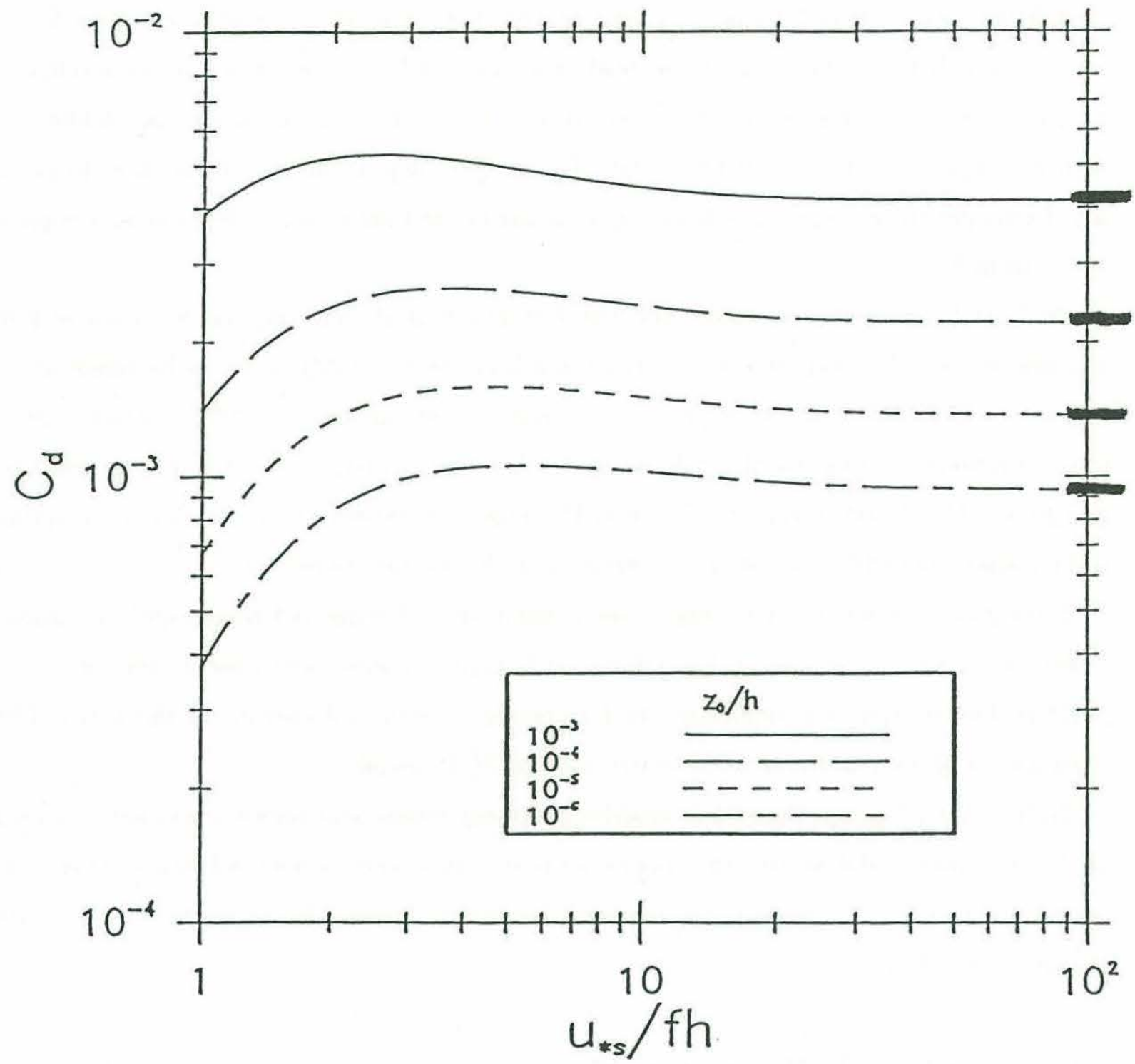

Figure 2-5: Variation of quadratic drag tensor magnitude as a function of Ekman depth to water depth ratio (from Jenter and Madsen, 1989). 
vertically integrated to obtain (2.1) and (2.2). Specifically,

$$
\begin{aligned}
& \frac{\partial u}{\partial t}-f v=-g \frac{\partial \zeta}{\partial x}+\frac{\partial}{\partial z}\left(\nu_{T} \frac{\partial u}{\partial z}\right) \\
& \frac{\partial v}{\partial t}+f u=-g \frac{\partial \zeta}{\partial y}+\frac{\partial}{\partial z}\left(\nu_{T} \frac{\partial v}{\partial z}\right)
\end{aligned}
$$

where $(u(z), v(z))$ is the depth-dependent horizontal velocity in the $(x, y)$ direction. Notice that the stresses are modelled as the product of an eddy viscosity, $\nu_{T}$, and the vertical shear in the velocity profile. Also, the hydrostatic assumption is made to relate the pressure gradient to the sea surface slope.

The key to resolving successfully the flow structure using (2.22) and (2.23) lies in choosing an appropriate form for the eddy viscosity. Since the $2 \frac{1}{2}$-D model is intended to describe winddriven flow in shallow water, the eddy viscosity must account for the presence of both surface and bottom boundary layers as well as for the possibility that the two overlap in sufficiently shallow water.

The DRM assumes

$$
\nu_{T}= \begin{cases}\kappa u_{* s}(h-z), & z_{m}<z \leq h \\ \kappa u_{* b} z, & 0 \leq z<z_{m}\end{cases}
$$

where $\kappa=0.4$ is von Karman's constant, $u_{* b}$ is the a priori unknown bottom shear velocity defined by $\left|\vec{\tau}_{b}\right| / \rho=u_{* b}^{2}$ and $z_{m}$ is a matching height (figure 2-6). The origin of the $z$-axis is chosen to coincide with the sea bottom as shown in figure 2-6. The matching height is defined by

$$
z_{m}=h \frac{u_{* b}}{u_{* s}+u_{* b}}
$$

a formulation which was first suggested by Madsen (1977).

It is important to note that the matching height is not the point where the two functions of (2.24) overlap. Such a choice would apportion a larger part of the water column to the function having the smaller shear velocity. The choice of matching height (2.25) ensures that each function is apportioned a part of the water column according to the relative strength of its shear velocity. Consequently, the eddy viscosity is discontinuous at $z=z_{m}$ (figure 2-6) unless $u_{* b}=u_{* s}$. Further implications of this discontinuity are discussed below.

The bilinear character of (2.24) is chosen for two reasons. First, in keeping with the efficiency goal of the $2 \frac{1}{2}$-D model, it does not preclude a simple analytical solution to $(2.22)$ and 


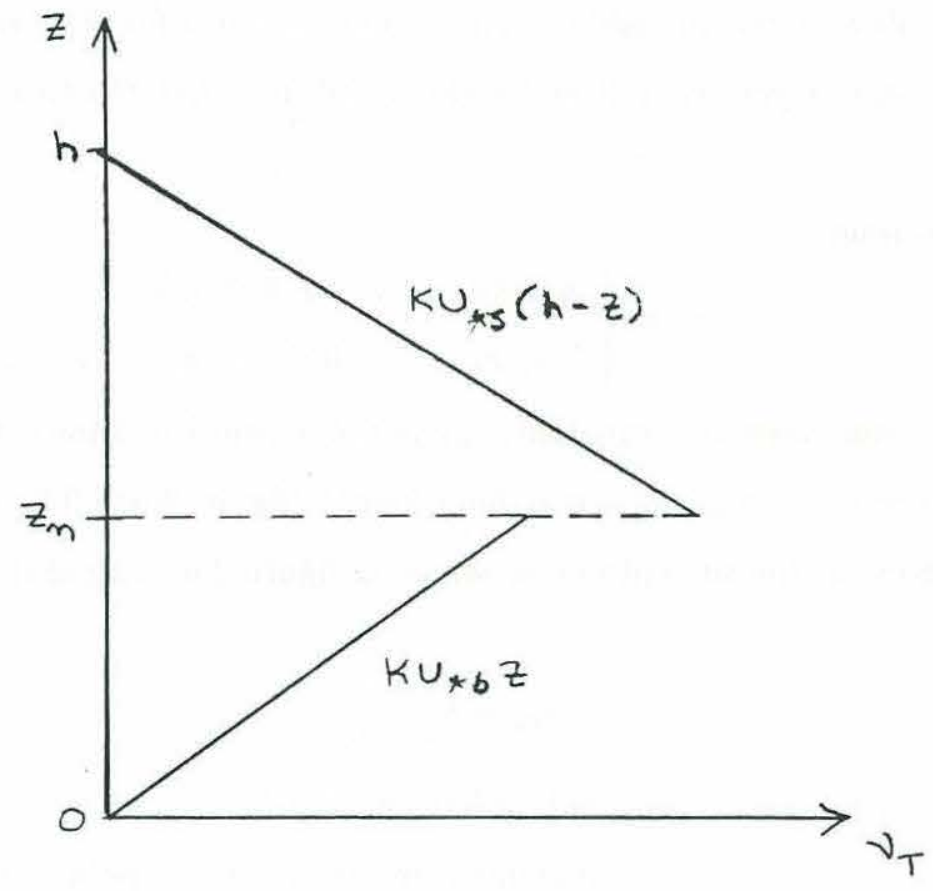

Figure 2-6: Schematic representation of the eddy viscosity. 
(2.23). As important as simplicity, however, is its ability to reproduce much of the surface and bottom boundary layer behavior observed in real flows. For example, wind-driven surface boundary layers in the laboratory have been shown to exhibit a log-deficit profile near the free surface (Shemdin, 1972; Wu, 1975). Log-deficit layers have also been observed in the field using near surface drifters (Churchill and Csanady, 1983; Csanady, 1984), current meter drifters (Richman et al., 1987) and platform mounted acoustic current meters (Terray et al., 1987). An approximation to log-deficit variation is produced by an eddy viscosity which increases linearly away from the free surface while the stress remains relatively constant.

Another characteristic of surface boundary layers which is dependent upon the eddy viscosity is the rotational behavior. Field experiments in deep water indicate a structure which is somewhat different from the classical Ekman spiral. One major difference is the angle which the surface velocity makes with the wind stress. Classical theory (Ekman, 1905) predicts that this angle should be $45^{\circ}$, while observations (e.g. Stacey et al., 1986) indicate that it may be substantially smaller. An eddy viscosity increasing linearly away from the free surface has been shown by Madsen (1977) to predict a reduction in angle between the wind and surface velocity.

Bottom boundary layer behavior has also been studied in both laboratory (e.g. Nezu and Rodi, 1986) and field experiments (see Soulsby, 1983; Grant and Madsen, 1986 for reviews.). For steady unstratified flow, the turbulent boundary layer near a solid wall is well known to contain a region of logarithmic variation with depth (Clauser, 1956). As with the surface log-deficit layer, this variation is approximated fairly accurately by an eddy viscosity which increases linearly away from the boundary while the stress profile remains relatively constant. In addition, the slope of the eddy viscosity has been shown to be proportional to the bottom shear velocity, $u_{* b}$.

Constant stress layers are clearly an approximation near the boundaries. Nonetheless, $(2.24)$ reproduces the basic near-boundary flow features. There is evidence (e.g. Businger and Arya, 1974) that the linear eddy viscosity should be cut off at a distance, $\gamma u_{*} / f$, away from the boundary where typical $\gamma$ values have been quoted in the range 0.04 to 0.06 (Lavelle and Mofjeld, 1983). The fact that the present analysis neglects the eddy viscosity cutoff implies that the resulting theory should be limited to shallow water only.

A working definition of "shallow water" is suggested by the results of Jenter and Madsen 
(1989) who found that the cutoff points of the surface and bottom boundary layers overlapped (i.e. $\gamma\left(u_{* s}+u_{* b}\right) / f \geq h$ ) for $u_{* s} / f h \geq 17$ when $\gamma=0.04$ and for $u_{* s} / f h \geq 11$ when $\gamma=0.06$. Further experimentation with a trilinear eddy viscosity (linearly connecting the cutoff points when they do not overlap) showed that the bilinear solutions were quantitatively robust above $u_{* s} / f h \approx 5$ for $\gamma=0.06$. Recent results obtained by Mofjeld (1988) for purely pressuredriven flow in deep water (i.e. $u_{* s}=0$ in (2.24)) indicate that differences in bottom stress magnitude with or without a cutoff are at most $10 \%$ for all reasonable flow conditions. Bottom stress directional variation is similarly small. Consequently, concern over application of the $2 \frac{1}{2}$-D model presented here should only be raised for "light winds or deep water" below say $u_{* s} / f h \approx 5$ (see table 2.1.), which is outside the range of primary interest.

The question of the applicability of (2.24) in the bottom stress prediction process becomes moot for low values of $u_{* s} / f h$ because bottom stress does not play an important dynamical role in the wind-driven DAM. Csanady (1984) uses simple scaling arguments to support the idea that bottom stress loses its dynamical importance when $u_{* s} / f h=\mathrm{O}(1)$. Likewise, the fairly elaborate turbulence closure model of Overland et al. (1984) predicts that wind-driven ice movement shows no bottom friction effects when $u_{* s} / f h$ is less than about 10.

Having defined the eddy viscosity and its range of applicability, a means of solving (2.22) and (2.23) is required. One potential choice is by Laplace transform methods. However, this is only possible for eddy viscosity profiles of particular forms. Jelesnianski (1970) and Forristall (1974) presented Laplace transform-like solutions relating bottom stress to the time histories of local wind stress and sea surface slope for wind-driven coastal flow with constant $\nu_{T}$. Madsen (1977) presented a Laplace transform solution of (2.22) and (2.23) in an infinitely deep ocean with a time-independent eddy viscosity that increased linearly away from the sea surface (i.e. the surface function of (2.24)). Unfortunately, these Laplace transform solutions are not applicable in the present study because the functional dependence of $\nu_{T}$ on the a priori unknown bottom stress implies that $\nu_{T}$ is unpredictably time-dependent.

An alternative is the decomposition of the velocity profile into vertical modes that are separable in depth and time. This procedure was first introduced by Heaps (1972) for a constant eddy viscosity. It was later modified for time- and depth-dependent eddy viscosity by both Nihoul (1977), Baker and Jordan $(1980,1981)$ and Jordan and Baker (1980). The procedure 
has since been used by a variety of investigators (e.g. Davies, 1987). Introduction of a modal structure reduces the full solution of (2.22) and (2.23) to the solution of first-order ordinary differential equations for the time-dependent mode amplitudes. However, this is only possible when the eddy viscosity is formulated as a separable function of depth and time. As with the Laplace transform solution, functional dependence of $\nu_{T}$ on the time-varying bottom stress makes the modal approach inapplicable because $\nu_{T}$ is not separable when $z_{m}$ is allowed to vary (i.e. the ratio of bottom stress to surface stress is allowed to vary).

Since Laplace transform and modal decomposition solutions are not possible, (2.22) and (2.23) must be simplified if an analytic solution is sought. One way to do this is by assuming that the local time derivatives in (2.22) and (2.23) are depth-independent and equal to their depth-averaged values (i.e. replace $\partial \vec{u} / \partial t$ in $(2.22)$ and $(2.23)$ by $(1 / h) \partial \vec{U} / \partial t)$. This permits $(2.22)$ and $(2.23)$ to be rewritten as

$$
\begin{aligned}
-f\left(v-v_{g}\right) & =\frac{\partial}{\partial z}\left(\nu_{T} \frac{\partial\left(u-u_{g}\right)}{\partial z}\right) \\
f\left(u-u_{g}\right) & =\frac{\partial}{\partial z}\left(\nu_{T} \frac{\partial\left(v-v_{g}\right)}{\partial z}\right)
\end{aligned}
$$

where

$$
\begin{aligned}
& u_{g}=-\frac{1}{f}\left(\frac{1}{h} \frac{\partial V}{\partial t}+g \frac{\partial \zeta}{\partial y}\right) \\
& v_{g}=\frac{1}{f}\left(\frac{1}{h} \frac{\partial U}{\partial t}+g \frac{\partial \zeta}{\partial x}\right)
\end{aligned}
$$

The subscript $g$ refers to the fact that $u_{g}$ and $v_{g}$ without the local time derivatives are merely geostrophic velocities. Equations (2.28) and (2.29) emphasize that the unsteady terms are treated as a modification to the pressure gradient with their effect being felt uniformly throughout the water column. Limitations on temporally resolvable processes caused by this approximation are discussed later.

Equations (2.26) and (2.27) are rewritten by taking their dot product with $(1, i)(i=\sqrt{-1})$ to yield

$$
\frac{d}{d z}\left(\nu_{T} \frac{d \mathcal{W}}{d z}\right)-\text { if } \mathcal{W}=0
$$

where $\mathcal{W}=\left(u-u_{g}\right)+i\left(v-v_{g}\right)$ is the complex difference between the total velocity and $\left(u_{g}, v_{g}\right)$. Inserting (2.24) into (2.30) yields

$$
\frac{d}{d z}\left(\kappa u_{* s}(h-z) \frac{d \mathcal{W}}{d z}\right)-i f \mathcal{W}=0, \quad z_{m} \leq z \leq h
$$




$$
\frac{d}{d z}\left(\kappa u_{* b} z \frac{d \mathcal{W}}{d z}\right)-i f \mathcal{W}=0, \quad 0 \leq z \leq z_{m}
$$

Solutions to (2.31) and (2.32) are found in many applied mathematics texts (e.g. Hildebrand, 1976). They are

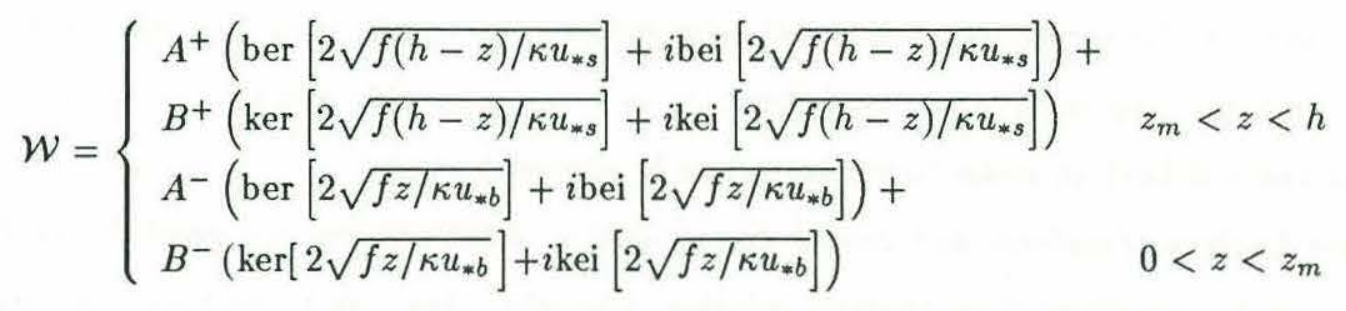

where $A^{ \pm}$and $B^{ \pm}$are complex constants determined by surface and bottom boundary conditions and ber, bei, ker, and kei are zero-order Kelvin functions.

The boundary conditions for determining $A^{ \pm}$and $B^{ \pm}$are

$$
\begin{gathered}
\left.\mathcal{W}\right|_{z=z_{0}}=-\left(u_{g}+i v_{g}\right) \\
\lim _{z \rightarrow h} \nu_{T} \frac{d \mathcal{W}}{d z}=\frac{\tau_{s x}+i \tau_{s y}}{\rho}
\end{gathered}
$$

where the complex forcing quantities, $\left(u_{g}+i v_{g}\right)$ and $\left(\tau_{s x}+i \tau_{s y}\right)$, are passed to the DRM from the DAM.

Equation (2.34) is merely a no-slip condition applied a small distance, $z_{0}$, above the sea bottom. This is necessary because (2.33) behaves logarithmically as $z \rightarrow 0$. In reality, $z_{0}$, the roughness length, is a function of sediment characteristics, bed configuration, and near-bottom turbulence structure (e.g., due to wave-current interaction). However, the analysis presented here assumes that $z_{0}$ is known a priori, thereby ignoring details of near-bottom flow structure and bottom roughness in the determination of roughness length.

Condition (2.35) is a statement relating known surface shear stress to unknown surface shear. It is necessary to take the limit as the boundary is approached since the velocity profile behaves logarithmically and thus the shear profile varies as $1 /(h-z)$. The stress limit is finite, however, since $\nu_{T}$ approaches 0 in such a way that it balances the shear as it approaches $\infty$.

Supplementing (2.34) and (2.35) are matching conditions at the level of discontinuity in the eddy viscosity.

$$
\left.\mathcal{W}\right|_{z=z_{m+}}=\left.\mathcal{W}\right|_{z=z_{m-}}
$$




$$
\left.\nu_{T} \frac{d \mathcal{W}}{d z}\right|_{z=z_{m+}}=\left.\nu_{T} \frac{d \mathcal{W}}{d z}\right|_{z=z_{m-}}
$$

Matching conditions (2.36) and (2.37), respectively, ensure continuity of velocity and shear stress across $z_{m}$. Because the shear stress is continuous and the eddy viscosity is discontinuous at $z_{m}$ the shear must be discontinuous there. This means the velocity profile is kinked at $z_{m}$. Clearly, a kink is unrealistic. However, it is not pronounced so long as $z_{m} \gg z_{0}$, which is normally the case. Comparison between bilinear eddy viscosity results and those for a continuous trilinear eddy viscosity mentioned earlier show little effect on the velocity profile, its depth-average or the associated bottom stress.

While boundary conditions (2.34), (2.35) and matching conditions (2.36), and (2.37) determine $A^{ \pm}$and $B^{ \pm}$, the problem is still not closed ${ }^{2}$. The bottom shear velocity, $u_{* b}$, is a priori unknown and part of the solution. Therefore, the solution must be iterated to satisfy an additional compatibility condition

$$
\lim _{z \rightarrow 0} \nu_{T}\left|\frac{d \mathcal{W}}{d z}\right|=\frac{\left|\tau_{b x}+i \tau_{b y}\right|}{\rho} \equiv u_{* b}^{2}
$$

Satisfaction of (2.38) ensures that the bottom stress magnitude on which the eddy viscosity is based is the actual bottom stress magnitude produced by the current profile. Unlike most vertical profile models, both no-slip (2.34) and shear (2.38) boundary conditions are satisfied.

The idea of using a turbulent mixing scheme based on an a priori unknown bottom shear velocity is not new. Thomas (1975) employed a similar assumption, but did not incorporate a surface mixing formulation. Thomas' analysis stressed the need for an improved eddy viscosity formulation that accounts for surface boundary layer as well as overlapping boundary layer effects in shallow wind-driven waters. In addition to Thomas' analysis, similar iterative solution procedures have been employed for boundary layer models of wave-current interaction (e.g. Smith and Long, 1976; Smith, 1977; and Grant and Madsen, 1979).

Figure 2-7 shows a schematic representation of the solution procedure for the DRM as it is employed in the $2 \frac{1}{2}-\mathrm{D}$ model. The iterative nature of the solution is apparent. This reduces the computational efficiency of the DRM, but experience has shown only 5 or 6 iterations are required to obtain agreement in $u_{* b}$ to less than $0.1 \%$. Upon completion of iteration, the bottom

\footnotetext{
${ }^{2}$ Complete expressions for $A^{ \pm}$and $B^{ \pm}$are given by Jenter (1987).
} 
stress and velocity profile (and, consequently, the transport ${ }^{3}$ ) are obtained. The bottom stress and transport are then manipulated to yield estimates of $r$ and $\theta$ to be used in the DAM.

Before describing the $2 \frac{1}{2}$-D model, a brief discussion of the obvious numerical alternative to an analytic DRM should be presented. Clearly, a numerical procedure is adaptable to the eddy viscosity (2.24), and eliminates the problems with Laplace transforms and modal decomposition. It can also handle depth-dependence of the local time derivatives giving it an advantage over the Kelvin function solution. However, use of a numerical procedure in the context of a $2 \frac{1}{2}$-D model raises the question of its efficiency. To examine this issue, steady-state (i.e. dropping $\partial \vec{u} / \partial t$ ) solutions of (2.22) and (2.23) were calculated analytically and numerically and the results compared for speed and accuracy. The numerical solution consisted of a centered difference scheme on a logarithmically stretched grid with fine spacing near the surface and bottom in order to resolve the boundary layers without restrictively small grid spacing in the interior of the water column. The problem was formulated as a boundary value problem and solved by a standard tri-diagonal matrix inversion. Depth-averaging of the resultant solution was done by Simpson's rule. Additional details of the numerical procedure are given by Jenter (1987).

Table 2.3 summarizes the comparison. One hundred runs with random wind stresses, pressure gradients, bottom roughnesses and water depths were performed and statistics of the results calculated. The table shows that for the numerical and analytical solutions to have similar computation times, the numerical solution can utilize only 25 grid points. When this is the case, however, the relative accuracy of the numerical scheme is terrible. While the numerical and analytical bottom stress magnitudes agree in the mean, two standard deviations represent $\pm 53 \%$. This can be reduced to $\pm 8 \%$ at a cost of 4 times the CPU time and to $\pm 2 \%$ at 20 times the CPU time. Given these results and the fact that the DRM is the most CPU-intensive part of the $2 \frac{1}{2}$-D model, a numerical solution to $(2.22)$ and $(2.23)$ is rejected on efficiency grounds.

\subsection{The $2 \frac{1}{2}-\mathrm{D}$ Model}

Together the DAM and DRM provide a wind-driven coastal circulation model with an improved estimate of bottom stress. The basic $2 \frac{1}{2}$-D modelling scheme is illustrated by the following

\footnotetext{
${ }^{3}$ A complete analytic expression for the transport is given by Jenter (1987).
} 


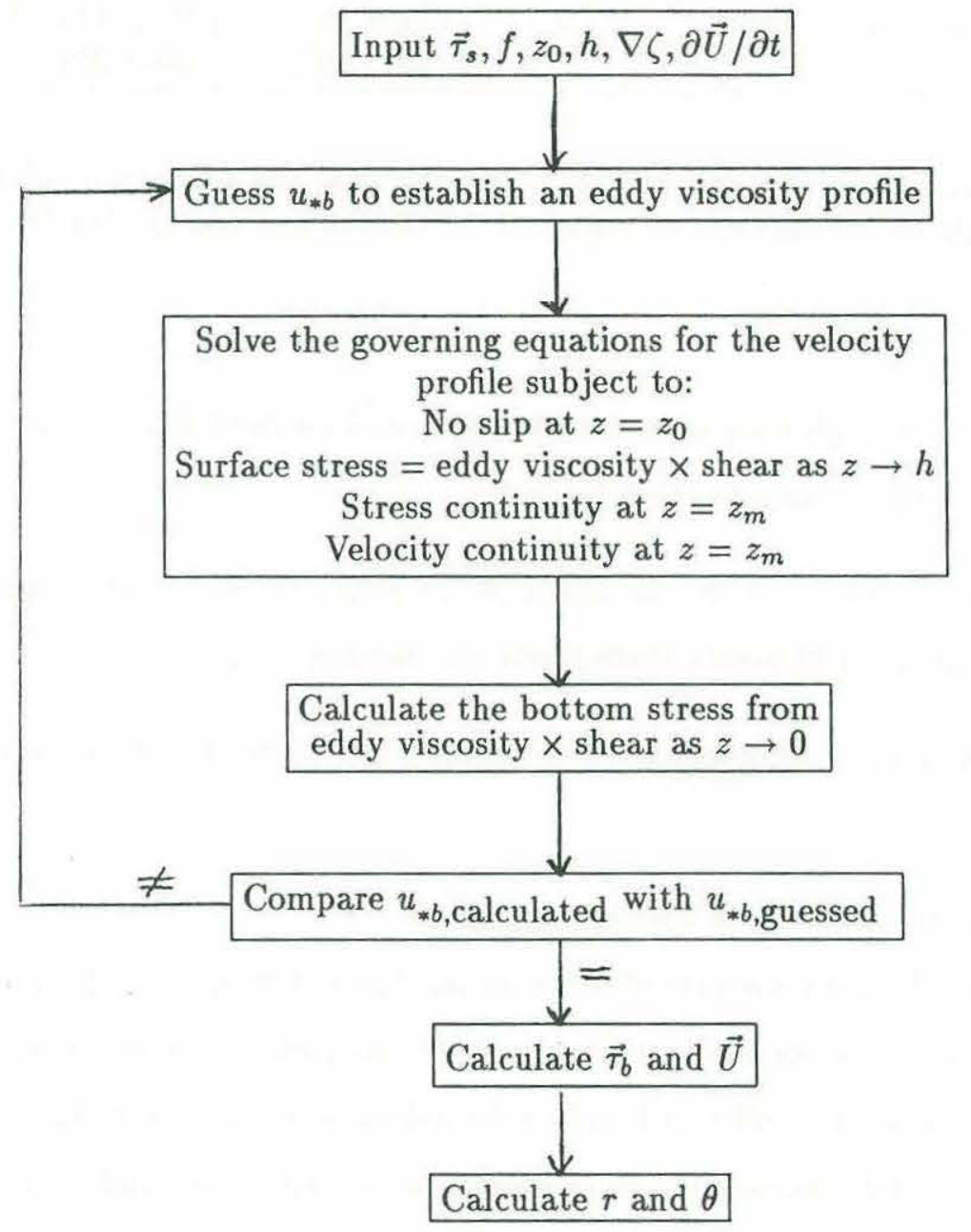

Figure 2-7: Schematic of the depth-resolving model 


\begin{tabular}{|c|c|c|c|}
\hline case & CPU time & $\frac{\left|\vec{r}_{b}\right|}{\left|\vec{r}_{b}\right|}$ & $\frac{|\vec{U}|}{|\vec{U}|_{\text {analytical }}}$ \\
\hline Analytical & $0: 02.6$ & 1 & 1 \\
\hline Numerical 25pts & $0: 02.4$ & $\begin{array}{c}1.04 \pm .53 \\
(.77-2.7)\end{array}$ & $\begin{array}{l}1.00 \pm .18 \\
(.85-1.45)\end{array}$ \\
\hline Numerical 100pts & $0: 08.9$ & $\begin{array}{l}1.00 \pm .08 \\
(.94-1.30)\end{array}$ & $\begin{array}{l}1.00 \pm .04 \\
(.97-1.10)\end{array}$ \\
\hline Numerical 500 pts & $0: 43.2$ & $\begin{array}{l}1.00 \pm .02 \\
(.99-1.06)\end{array}$ & $\begin{array}{c}1.00 \pm .008 \\
(.99-1.02)\end{array}$ \\
\hline
\end{tabular}

Table 2.3: Comparison of speed and accuracy for analytical and numerical solutions with random forcings. Statistics are mean \pm two standard deviations and (range) for 100 runs.

simple procedure:

- step the DAM through time using a time step based on the CFL stability condition until a decision is made to update the drag tensor

- pass forcing information from the DAM to the DRM so that drag tensor estimates can be obtained at the grid points throughout the domain

- pass the drag tensor estimates from the DRM back to the DAM and return to the first step above

This process is illustrated in more detail in figure 2-8.

The $2 \frac{1}{2}$-D model actually consists of three parts. The DAM and DRM have been described in sections 2.1 and 2.2 , respectively, while the third part, the roughness model, is mentioned merely for completeness. In reality, it is only a formalism at present since the roughness field is specified a priori and left unchanged. Nonetheless, the model is formulated in such a way that the roughness field can be updated whenever the DRM is run. This is done with an eye toward the possibility of including such roughness altering processes as wave-current interaction (e.g. Grant and Madsen, 1979) and moveable bed effects (e.g. Grant and Madsen, 1982) in future versions.

The first step in running the $2 \frac{1}{2}$-D model is initialization of the field variables, $U, V, \zeta, r$ and $\theta$. All of these are set equal to 0 when the model is spun up from rest. In addition, the physical 
DAM

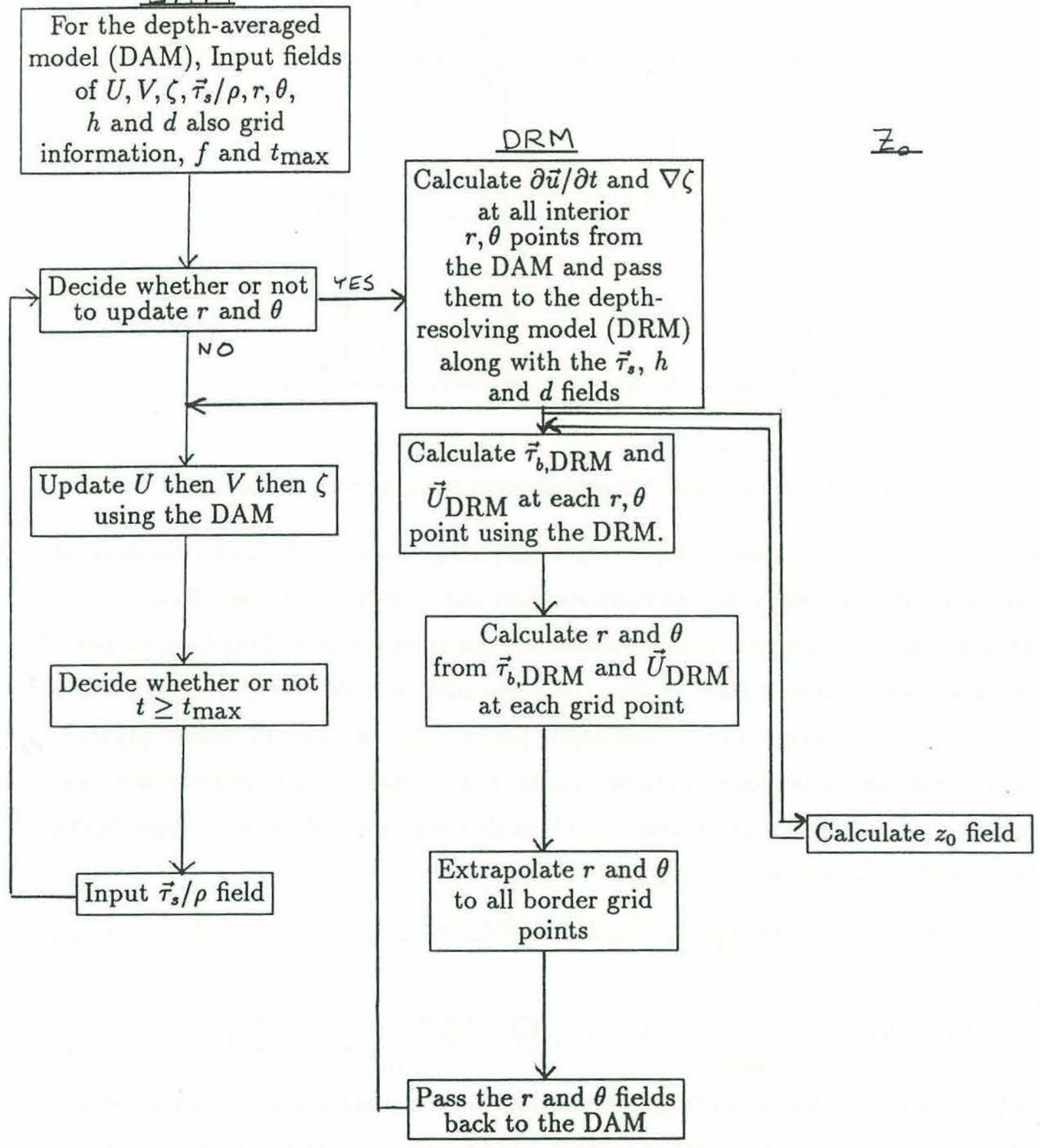

Figure 2-8: Schematic of the full $2 \frac{1}{2}-\mathrm{D}$ model. 


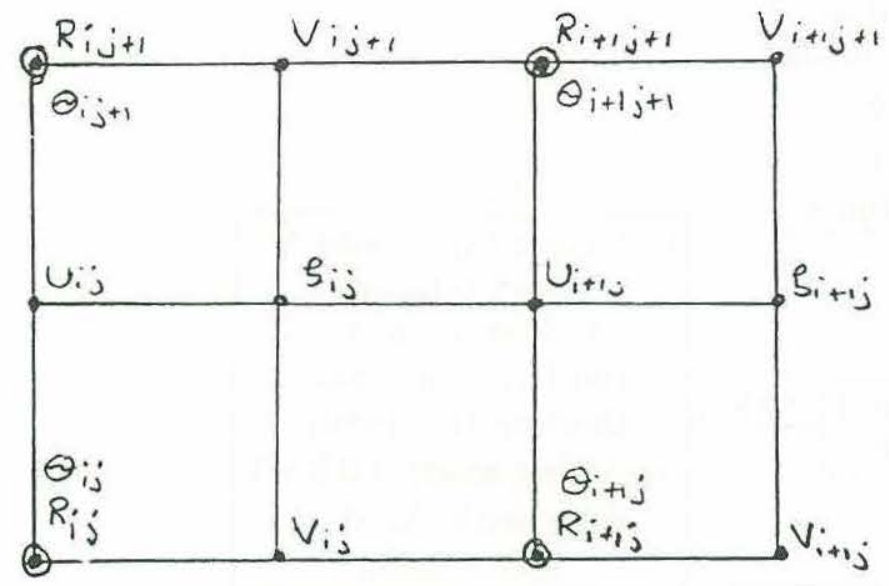

Figure 2-9: The Arakawa " $\mathrm{C}$ " grid showing superimposed $r, \theta$ nodes.

configuration of the model must be input, which includes an Arakawa "C" grid superimposed over the desired topography, a bottom roughness field and an initial wind stress field.

After initialization, the DAM is time stepped forward using the forward/backward scheme. Following a predetermined number of time steps, the DRM is called to update the $r$ and $\theta$ fields. The update is performed at the unoccupied fourth corner of each grid element (figure 29 ) because calculation of the pressure gradient needed to force the DRM is straightforward using the surrounding $\zeta$ points as is calculation of the local time derivatives of transport. Specifically, at the $r, \theta$ node of the grid element $i, j$,

$$
\nabla \zeta=\frac{\zeta_{i, j}-\zeta_{i-1, j}+\zeta_{i, j-1}-\zeta_{i-1, j-1}}{2 \Delta x} \mathbf{i}+\frac{\zeta_{i, j}-\zeta_{i, j-1}+\zeta_{i-1, j}-\zeta_{i-1, j-1}}{2 \Delta x} \mathbf{j}
$$

and

$$
\frac{\partial \vec{U}}{\partial t}=\frac{U_{i, j}^{n}-U_{i, j}^{n-m}+U_{i, j-1}^{n}-U_{i, j-1}^{n-m}}{2 m \Delta t} \mathbf{i}+\frac{V_{i, j}^{n}-V_{i, j}^{n-m}+V_{i-1, j}^{n}-V_{i-1, j}^{n-m}}{2 m \Delta t} \mathbf{j}
$$

where $(\mathbf{i}, \mathbf{j})$ are unit vectors in the $(x, y)$ directions and $m$ is the number of time steps between drag tensor updates. Clearly (2.39) and (2.40) cannot be applied at the DAM boundaries. Therefore, $\nabla \zeta, \partial \vec{U} / \partial t, r$ and $\theta$ are calculated only at interior grid elements.

Once fields of $\nabla \zeta$ and $\partial \vec{U} / \partial t$ (thus $u_{g}$ and $v_{g}$ from (2.28) and (2.29)) are calculated, the feedback from the DAM to the DRM at each node is specified. The DRM is forced by $\left(u_{g}+i v_{g}\right)$ along with the same wind stress and topography as used in the DAM. After importing the forcing, the roughness field, which is kept constant here, is input. 
Given the forcing, topography and roughness at each interior $r, \theta$ node, the DRM is applied subject to the boundary conditions (2.34), (2.35), (2.36), (2.37), and (2.38). After convergence, the bottom stress and velocity profile are calculated from (2.38) without the absolute value signs and (2.33), respectively. The velocity profile is then integrated to yield a transport vector.

The bottom stress and transport predicted by the DRM are transformed into estimates of $r$ and $\theta$ using definitions derived from (2.11) and figure 2-1. Since forcings are only supplied at interior $r, \theta$ nodes, the DRM results are extrapolated to border nodes. Extrapolation to the boundary nodes is done linearly from the two nearest $r, \theta$ points along a line intersecting the node and perpendicular to the boundary. Corner extrapolations take into account the presence of both boundaries. After extrapolation, the complete field of drag tensor values is passed back to the DAM. Time stepping of the DAM continues until it is necessary to update the drag tensor field again.

It should be noted that the DAM requires $r$ and $\theta$ information at $U$ and $V$ nodes while the DRM provides it at $r, \theta$ nodes. Therefore, an intermediate spatial averaging takes place in order to provide drag tensor values at the proper locations. At the $U_{i, j}$ node:

$$
\begin{aligned}
(\tilde{f}-f) h & =r \sin \theta=\left(r_{i, j} \sin \theta_{i, j}+r_{i, j+1} \sin \theta_{i, j+1}\right) / 2 \\
\tilde{r} & =r \cos \theta=\left(r_{i, j} \cos \theta_{i, j}+r_{i, j+1} \cos \theta_{i, j+1}\right) / 2
\end{aligned}
$$

and at the $V_{i, j}$ node:

$$
\begin{aligned}
(\tilde{f}-f) h & =r \sin \theta=\left(r_{i, j} \sin \theta_{i, j}+r_{i+1, j} \sin \theta_{i+1, j}\right) / 2 \\
\tilde{r} & =r \cos \theta=\left(r_{i, j} \cos \theta_{i, j}+r_{i+1, j} \cos \theta_{i+1, j}\right) / 2
\end{aligned}
$$

Nothing has been said up to this point about choosing the frequency with which the drag tensor field is updated using the DRM. However, the overall efficiency of the $2 \frac{1}{2}$-D model clearly depends on the choice of update frequency. In fact, comparison tests show that the relative computational time between the DRM and DAM is roughly equal to $40 / m$ where $m$ is the number of time steps between updates. In other words, a run of the $2 \frac{1}{2}$-D model with $m=4$ spends 10 seconds of CPU time running the DRM for every 1 second of CPU time running the DAM.

A primary consideration when choosing the update frequency is that the bottom stress be able to respond appropriately to changes in forcing. There are only a few ways in which 


\begin{tabular}{||l|c||}
\hline Basin & Period, hr:min \\
\hline Lake Ontario & $4: 48$ \\
Lake Michigan & $9: 03$ \\
Lake Superior & $8: 00$ \\
Lake Erie & $14: 18$ \\
Narragansett Bay & $0: 44$ \\
San Francisco Bay & $1: 55$ \\
\hline
\end{tabular}

Table 2.4: Fundamental seiching periods which might be interpreted as $t_{\text {pressure }}$ for $2 \frac{1}{2}$-D model runs in the listed basin [after Wilson (1972)].

information about changes in forcing can be transmitted to the sea bottom. The mechanisms accounted for by the $2 \frac{1}{2}$-D model are propagation of pressure signals in the DAM and turbulent diffusion of surface stress in the DRM. Ideally, the update frequency should be chosen to resolve adequately the effect of these processes on drag tensor variation.

Time scales for the propagating pressure signals in the DAM are intimately dependent on the bottom and the geometry of the model domain. The fastest sea surface signals travel with the shallow water gravity wave speed, $\sqrt{g h}$, so their time scale can be thought of as being determined by the horizontal $(\mathcal{L})$ and vertical $(\mathcal{H})$ scales of the basin. Explicitly,

$$
t_{\text {pressure }} \propto \frac{\mathcal{L}}{\sqrt{g \mathcal{H}}}
$$

Examples of $\mathcal{L}$ might be the shelf width for coastal ocean flows or the basin diameter for circulation within enclosed or semi-enclosed bodies of water. The time scale defined by the latter is related to the familiar seiching period described in many textbooks (e.g. Lamb, 1953). Wilson (1972) presents a variety of observed fundamental seiching periods in well-known basins. Table 2.4 summarizes a few of these. Clearly, basin size and water depth greatly affect the seiching period.

Earlier, it was stated that the use of depth-independent time derivatives in the DRM constrains the maximum frequency for resolvable processes. Since the DRM does not accelerate different levels of the water column at different rates, it can only resolve processes which vary on a time scale longer than the time it takes shear stress related signals to diffuse throughout the water column. In other words, the water column, as modelled by the DRM, should be considered as a time-averager for processes with periods shorter than the turbulent diffusion 


\begin{tabular}{||c|c||}
\hline$u_{* s}, \mathrm{~cm} / \mathrm{s}$ & Diffusion time, hr:min \\
\hline 1 & $2 \mathrm{hrs}$ \\
2 & $1 \mathrm{hr}$ \\
4 & $30 \mathrm{~min}$ \\
\hline
\end{tabular}

Table 2.5: Diffusion times for various shear velocities in $20 \mathrm{~m}$ of water

time-scale. One estimate of this time scale can be made using simple scaling arguments (e.g. Csanady, 1984). If the diffusion rate is assumed to be proportional to the boundary shear velocity, $u_{*}^{2}=|\vec{\tau}| / \rho$, the time for a signal to traverse the water column is $\mathrm{O}\left(h / u_{*}\right)$.

The ratio of depth to shear velocity is a convenient but crude first estimate. Certainly a model which updates the drag tensor every $5 h / u_{*}$ is computationally more efficient than one which updates it every $h / u_{*}$. Therefore, a more specific time scale is sought. Madsen's (1977) Ekman layer model can be used. The model yields a solution for the spin-up of a surface Ekman layer due to an impulsively started constant wind stress over the open ocean. The model has an eddy viscosity that increases linearly away from the free surface with the same origin and slope as the upper function of (2.24). The resulting time- and depth-dependent velocity profile is given by

$$
u+i v=\frac{u_{* s}}{\kappa} \int_{0}^{t} \exp \left(\frac{-\hat{z}}{\kappa u_{* s} \tilde{t}}\right) \frac{\cos f \tilde{t}+i \sin f \tilde{t}}{\tilde{t}} d \tilde{t}
$$

where $\hat{z}$ is the distance from the free surface, and the wind stress is applied in the positive $x$-direction.

A diffusion time scale can be obtained from (2.46) by specifying a threshhold level for the velocity magnitude at $\hat{z}=h$. Reaching the threshhold should indicate that fluid at $\hat{z}=h$ is "aware" that a wind stress has been applied at $\tilde{z}=0$. The threshold is arbitrarily chosen here as $|u+i v|=u_{* s}$. In other words, it is assumed that the water column responds to changes in the wind stress in the time it takes the velocity at $\hat{z}=h$ to spin up from rest to a magnitude equal to the surface shear velocity. Although (2.46) appears complicated, the solution for $|u+i v|=u_{* s}$ is well approximated by

$$
\tilde{t} \approx t_{\text {diffusion }}=4 \frac{h}{u_{* s}}
$$

So the Ekman layer model suggests a time scale four times longer than $h / u_{* s}$. Example diffusion times calculated from (2.47) for $h=20 \mathrm{~m}$ are given in Table 2.5. 
Both pressure and diffusion time scales must be considered when choosing an update frequency. Comparison of tables 2.4 and 2.5 indicates that either one might set the update time scale. Once the time scale is set the number of time steps between updates is given by

$$
m=\frac{t_{\text {update }}}{\Delta t}
$$

It is important to note that the appropriateness of a particular choice of $t_{\text {update }}$ is subject to the reasonability of the $2 \frac{1}{2}$ - D model results it produces. 


\section{Chapter 3}

\section{$2 \frac{1}{2}$-D Model Results}

This chapter contains $2 \frac{1}{2}$-D model results for four different wind-driven coastal flow scenarios. Each has a domain geometry and wind stress field chosen to emphasize a particular aspect of the relationship between depth-averaged flow and bottom stress. The domains are periodic in the alongshore direction and bordered by a vertical drop-off to the deep ocean at a straight offshore boundary. This is done in order to minimize numerical boundary effects which might otherwise contaminate the results. The wind stress field is also chosen to be spatially uniform in all scenarios for the same reason.

The four scenarios are (figure 3-1):

1. a) Spin-up from rest of a constant depth shelf adjacent to a straight coast for a steady wind stress.

2. b) Spin-up from rest of a linearly-varying depth shelf adjacent to a straight coast for a steady wind stress.

3. c) Spin-up from rest of a domain with sinusoidal isobaths adjacent to a sinusoidal coast for a steady wind stress.

4. d) Response of a linearly-varying depth shelf adjacent to a straight coast for temporally periodic wind stress. 
a)

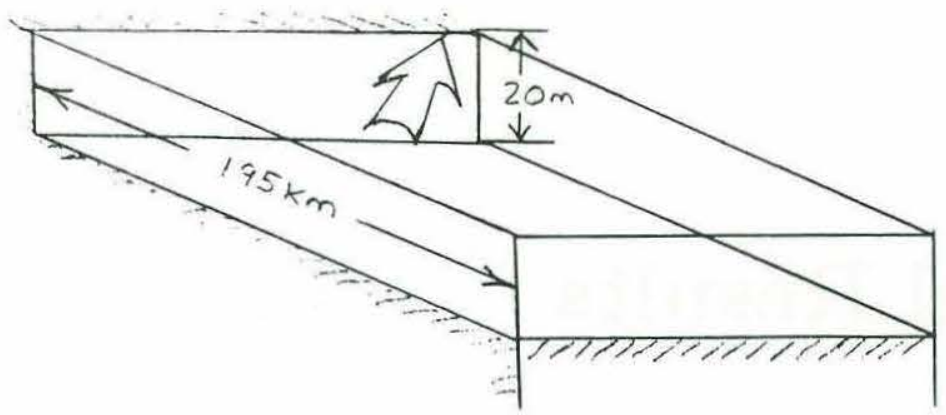

b)

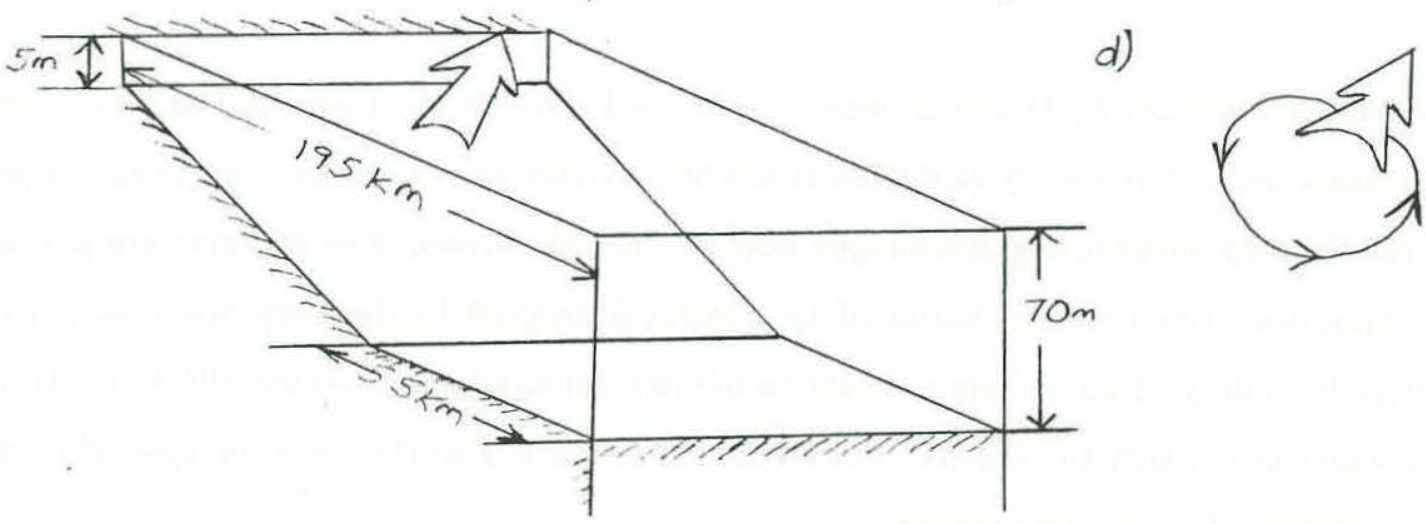

c)

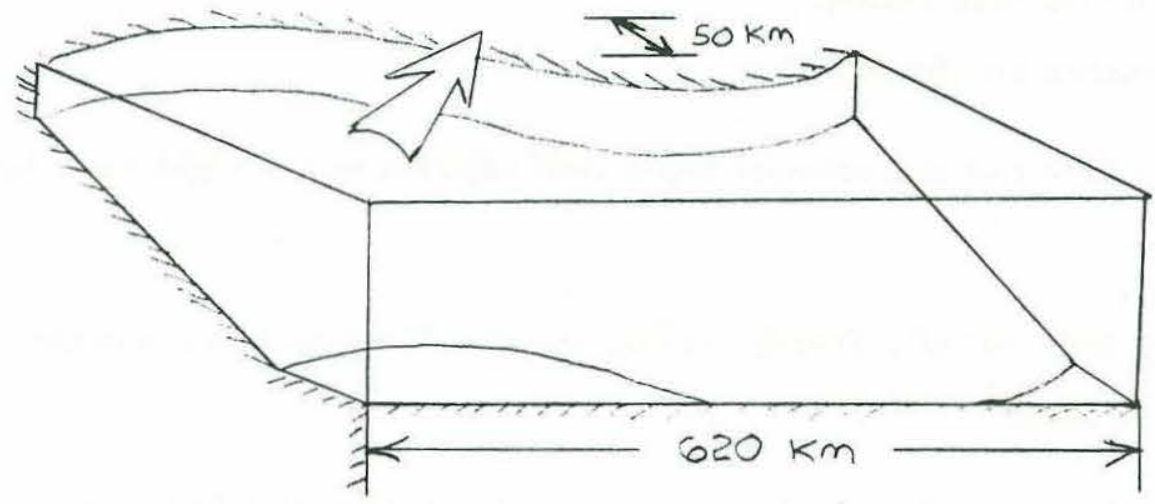

Figure 3-1: Schematic representation of the four scenarios investigated, a-d. 


\subsection{Constant Depth Shelf Adjacent to a Straight Coastline}

Flow over a flat shelf adjacent to a long straight coastline is the simplest scenario from which relevant conclusions can be drawn. These results are used both to introduce the physical phenomena encountered in wind-driven circulation near an open coast and to examine the basic behavior of the bottom stress - depth-averaged flow relationship.

\subsubsection{Model domain and wind stress field}

Specifically, this case employs a rectangular model domain (figure 3.1) forced by a wind stress applied uniformly over the region at time $t=0$ and held constant thereafter. The bathymetry is shallow and constant $(h=20 \mathrm{~m})$ between $y=0$ and $y=-L$. A no-flux condition perpendicular to the coastal boundary is imposed along $y=0$ and a clamped surface height condition is imposed along $y=-L$ (as if the domain suddenly became infinitely deep there). The shelf's cross-shore dimension is $195 \mathrm{~km}$ while its alongshore extent is $70 \mathrm{~km}$. The bottom is assumed to be covered by roughness elements with corresponding roughness lengths, $z_{0}=1 \mathrm{~cm}$. This value is chosen so as to represent a typical roughness length due to wave-current interaction. Lastly, the domain is overlain by an Arakawa " $\mathrm{C}$ " grid with a grid length, $\Delta x=10 \mathrm{~km}$. Choosing the grid size sets the CFL time step, as given by the righthand side of (2.21), to be $\Delta t_{\max }=505$ $s$ for all of the flat bottom runs. The actual model time step, however, is chosen as $90 \%$ of the CFL time step, or $455 \mathrm{~s}$, to help ensure stability.

The short alongshore extent of the domain is feasible because of the assumed alongshore uniformity in the response. Since periodic boundary conditions are implemented at the crossshore boundaries, end effects from improperly transmitted waves are eliminated.

While a wide flat shelf is clearly a drastic simplification of realistic shelf topographies, this first scenario illustrates much of the underlying physics of wind-driven flow near an open coast and emphasizes some differences between the $2 \frac{1}{2}$-D model and traditional bottom stress formulations. More realistic bathymetries and wind stress fields are examined later.

Three wind stress magnitudes are considered in this first scenario. The strongest corresponds to a wind speed of $33 \mathrm{~m} / \mathrm{s}$ ( 63 knots) at $10 \mathrm{~m}$ above the sea surface. This yields a value of $u_{* s} / f h=30$ (see Table 2.1). The intermediate strength wind stress corresponds to a wind speed of $14 \mathrm{~m} / \mathrm{s}$ (27 knots) yielding a value of $u_{* s} / f h=10$. The weakest wind stress corresponds 
to a wind speed of $8 \mathrm{~m} / \mathrm{s}$ (15 knots) yielding $u_{* s} / f h=5$. These cases will be referred to as the shallow, intermediate and deep cases respectively. This is in reference to the relationship between the surface boundary layer thickness and the water depth, not the water depth itself which is constant.

The diffusion time scales, as determined by (2.47), are 1250, 3750 and 7500 s. In all cases, the diffusion time scale is shorter than the pressure time scale, $L / \sqrt{g h}=13900$ s. Therefore, the vertical diffusion times set the update frequencies. The update frequencies used are every 5,8 and 16 time steps for the shallow, intermediate and deep cases, respectively.

In order to introduce the various phenomena efficiently, a case with both alongshore and cross-shore wind components is considered. Until specified later, all of the results presented are for a wind stress directed onshore at an angle of $45^{\circ}$ (measured counter-clockwise from the $x$-axis).

\subsubsection{Surface height and depth-averaged velocity}

Figure 3-2 shows time series of sea surface height for the three wind strengths. Significant variation among the subplots illustrates clearly that the magnitude of the wind stress has a large effect on sea surface response. Notably, there are differences in both the transient and steady-state behavior of the flow.

Roughly speaking, there are two kinds of time-dependent behavior depicted in figure 3-2. The first has a long smooth variation relative to the second and represents the overall approach to steady-state. The second has a shorter more oscillatory variation and represents the wavelike adjustment process accompanying the spin-up. Both types of motions are present in the three cases, but to different extents.

The spin-up consists of a short initial time period over which the surface remains flat. This lasts at each point until the arrival of the signal propagating outward from the coast. The signal travels with the long wave phase speed, $c=\sqrt{g h}=14 \mathrm{~m} / \mathrm{s}$. Therefore, the time for it to reach a particular point is a function of that point's distance from the coast. For the onshore point (located $75 \mathrm{~km}$, or $7.5 \Delta x$, from the coast), the time should be $75000 \mathrm{~m} / 14 \mathrm{~m} / \mathrm{s}=5360 \mathrm{~s} \approx 1.5$ hrs. For the offshore point (located $135 \mathrm{~km}$, or $135 \Delta x$, from the coast), it should be 135000 $\mathrm{m} / 14 \mathrm{~m} / \mathrm{s}=9640 \mathrm{~s} \approx 2.7 \mathrm{hrs}$. It is difficult to check this in figure $3-2$. However, the actual 

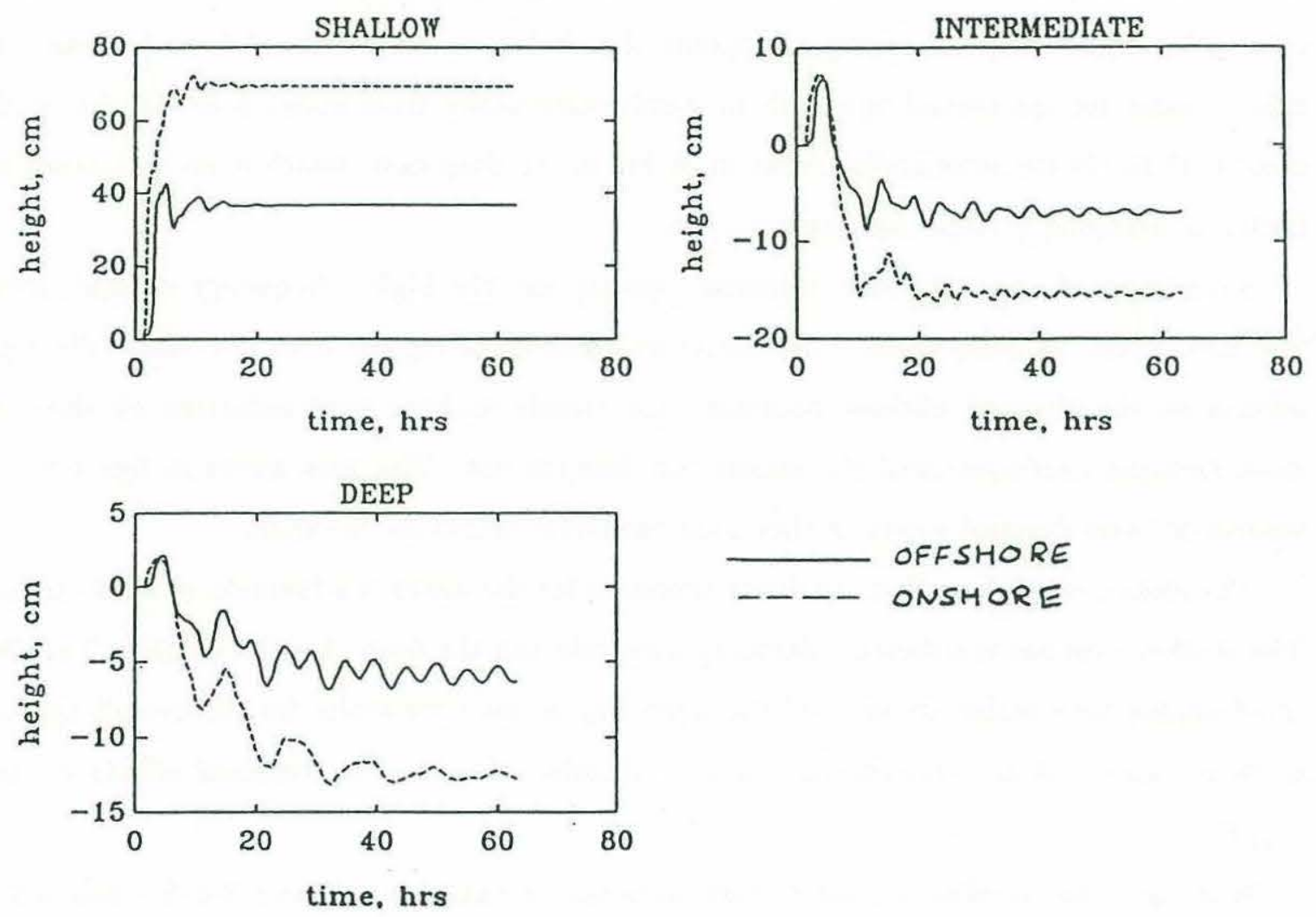

Figure 3-2: Time series of surface height at two different points across the shelf. The point labelled "inshore" is $75 \mathrm{~km}(7.5 \Delta x)$ from the shore and the point labelled "offshore" is $135 \mathrm{~km}$ $(13.5 \Delta x)$ from the shore. 
numerical results show these times to be correct. It is possible to see that the onshore point in each case is the first to show a non-zero height.

Once the surface height becomes non-zero, all three cases experience an initial set-up as the cross-shore component of wind stress moves water onshore. Overall rising of the surface continues only in the shallow case. In the intermediate and deep cases, the set-up eventually stops and water begins to move offshore producing a steady-state set-down. The reason for this is that, at steady-state, the Coriolis term predominates in the cross-shore momentum balance for the intermediate and deep cases while the wind stress term predominates in the shallow case. The surface slope is merely a response that balances the dominant term in each. The time it takes for the overall approach to steady-state varies from about $5 \mathrm{hrs}$ in the shallow case to $10 \mathrm{hrs}$ in the intermediate case to $20 \mathrm{hrs}$ in the deep case, which is an indication that frictional damping is larger for larger $u_{* s} / f h$.

Superimposed upon the long timescale spin-up are the higher frequency coastal seiches. The first "crest" of these waves is the initial set-up propagating out from the coast. The signal reflects off the clamped offshore boundary and travels onshore until reflecting off the coast. Reverberation continues until the seiches are damped out. The time series in figure $3-2$ are records of these damped waves as they pass particular points on the shelf.

The plots clearly show that the decay timescale for the waves is a function of wind strength. The shallow case has the shortest damping timescale and the deep case the longest. Therefore, the damping time scales are ordered the same way as the time scales for the overall approach to steady-state. Both relationships are an indication of enhanced frictional effects for large $u_{* s} / f h$.

It is easier to visualize the sea surface response by examining figures $3-3-3-5$ and $3-6-3-8$ instead of 3-2. Here, surface height is presented as a function of cross-shore distance and time. The first group of figures displays the information as contour lines of equal surface elevation and the second as a three dimensional surface formed by the juxtaposition of "snapshots" of cross-shore sea surface profiles.

Figures 3-3-3-5 show very clearly the initial set-up and propagation of the signal away from the coast at early times. The speed of propagation can roughly be deduced by taking the slope of one of the leftmost contour lines. For instance, the $10 \mathrm{~cm}$ contour line in figure $3-3$ has a 


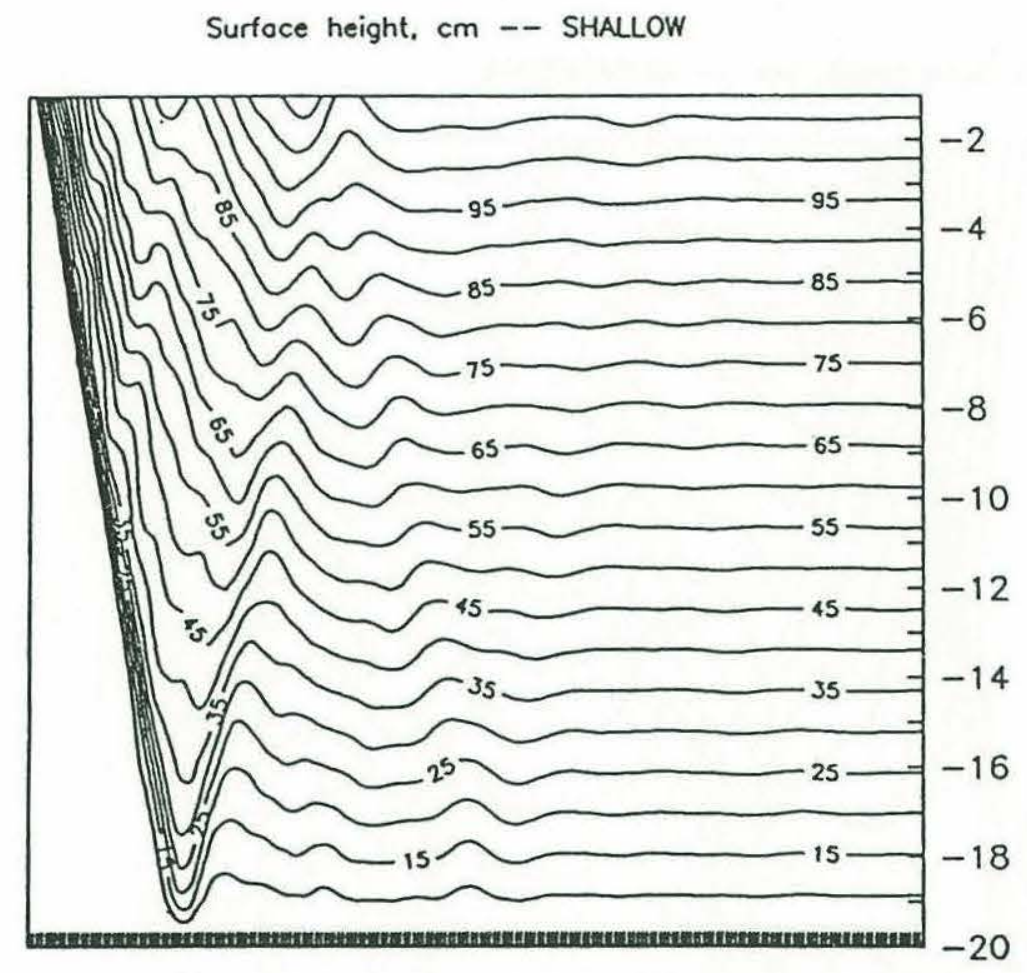

Figure 3-3: Contours of sea surface height in $y-t$ space for the shallow case. Tic mark spacing is $\Delta t$ on the horizontal axis and $\Delta x$ on the vertical axis. 
Surfoce height, $\mathrm{cm}$-- INTERMEDLATE

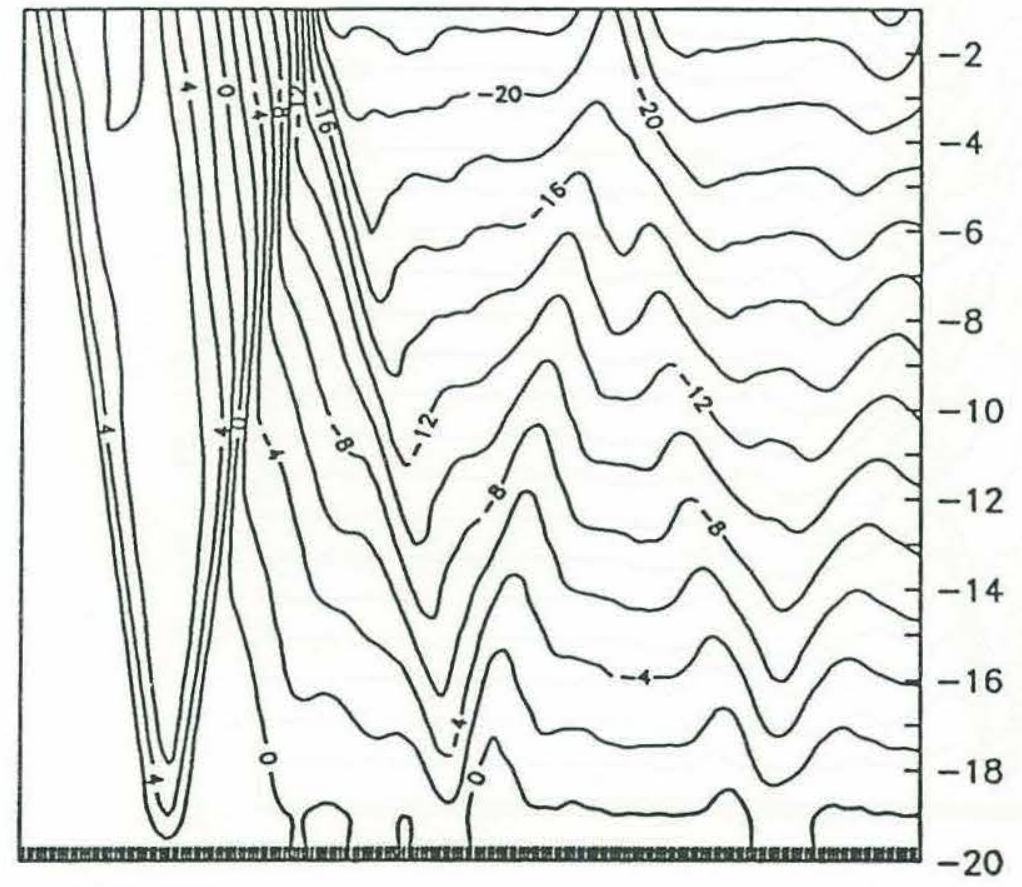

Figure 3-4: Contours of sea surface height in $y-t$ space for the intermediate case. Tic mark spacing is $\Delta t$ on the horizontal axis and $\Delta x$ on the vertical axis. 


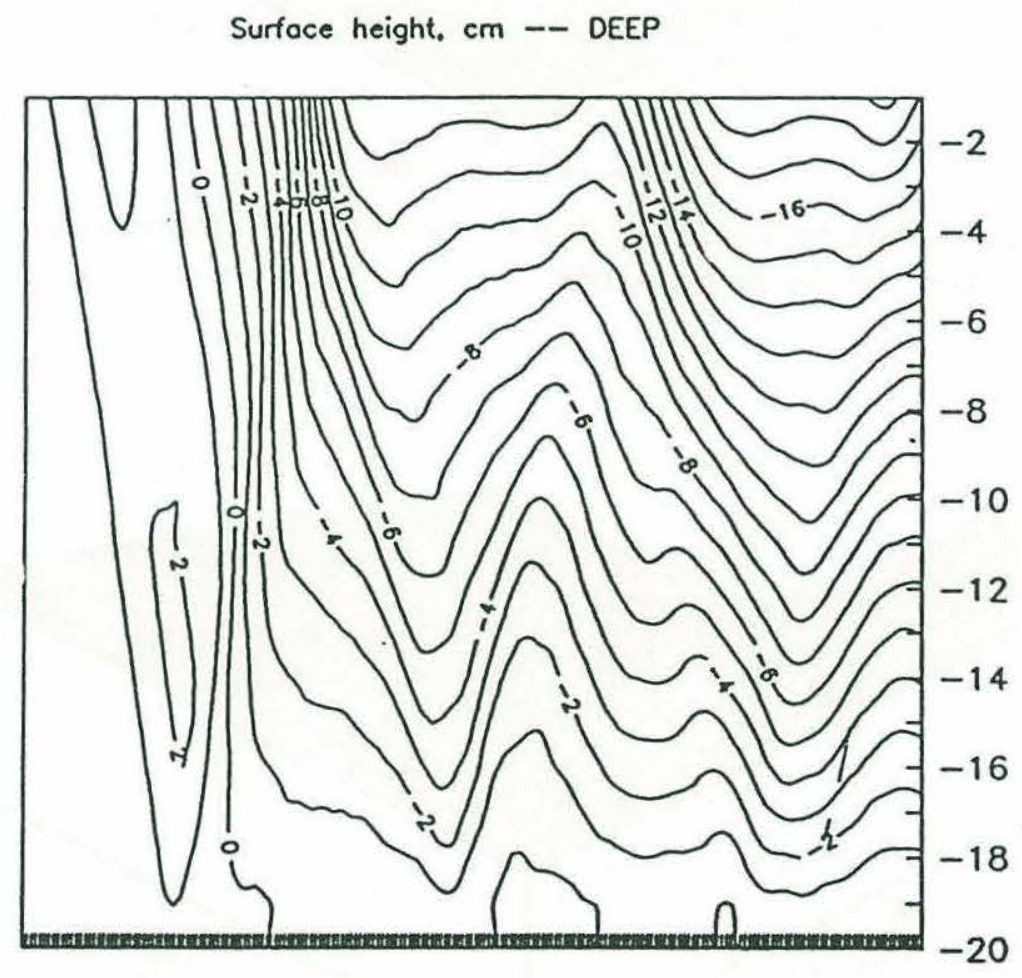

Figure 3-5: Contours of sea surface height in $y-t$ space for the deep case. Tic mark spacing is $\Delta t$ on the horizontal axis and $\Delta x$ on the vertical axis. 


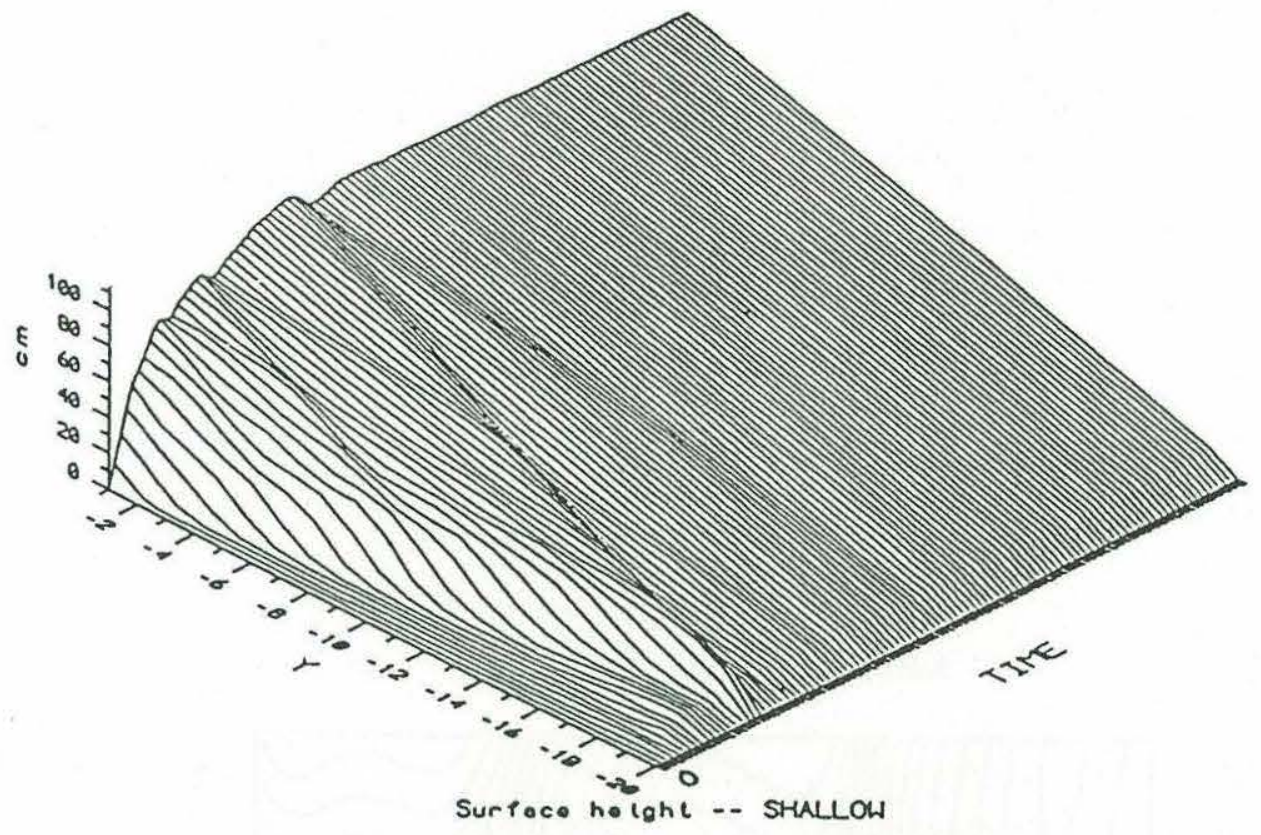

Figure 3-6: 3-D plot of sea surface height in $y-t$ space for the shallow case. Tic mark spacing is $\Delta t$ on the time axis and $\Delta x$ on the $\mathrm{y}$ axis.

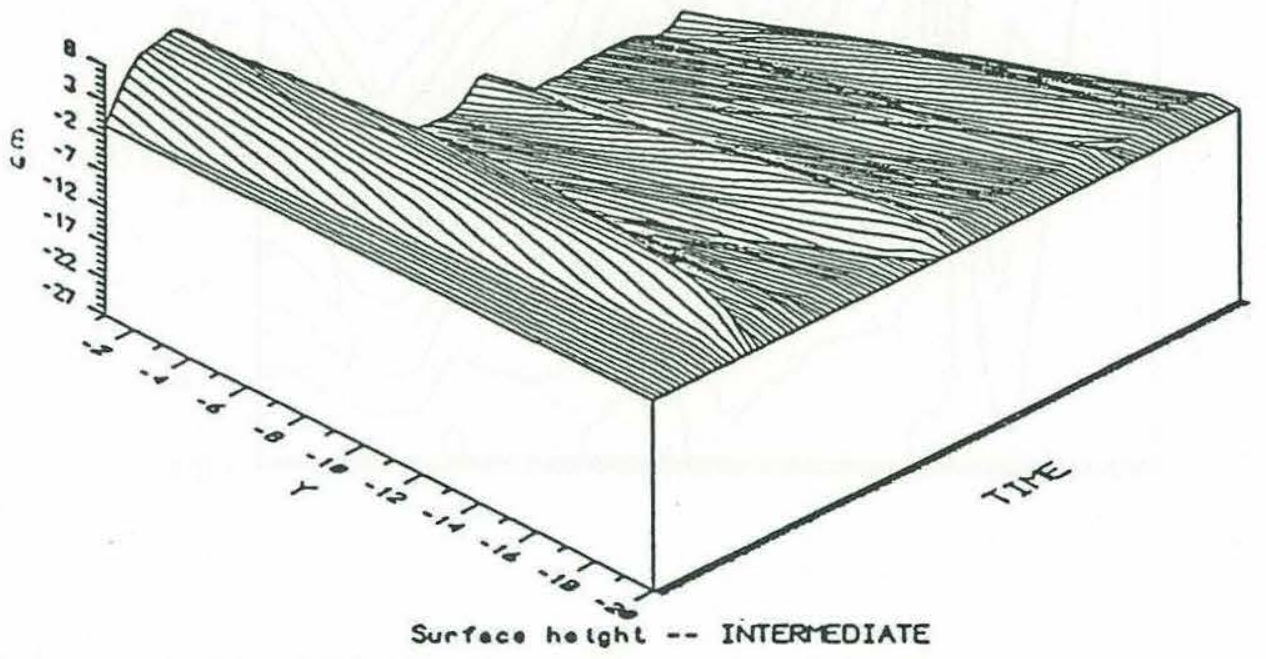

Figure 3-7: 3-D plot of sea surface height in $y-t$ space for the intermediate case. Tic mark spacing is $\Delta t$ on the time axis and $\Delta x$ on the y axis. 


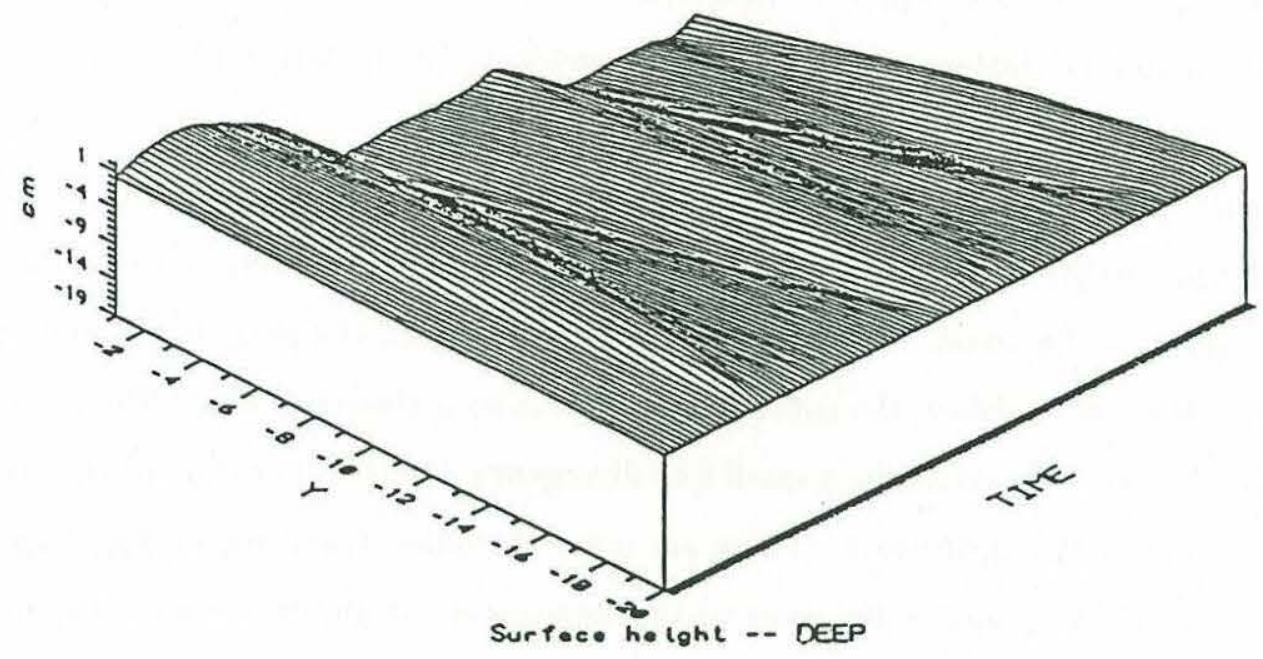

Figure 3-8: 3-D plot of sea surface height in $y-t$ space for the deep case. Tic mark spacing is $\Delta t$ on the time axis and $\Delta x$ on the $\mathrm{y}$ axis.

slope of about $190 \mathrm{~km} / 30 \Delta t$ yielding a propagation speed of $13.9 \mathrm{~m} / \mathrm{s}$. This is very close to the theoretical speed, $14.0 \mathrm{~m} / \mathrm{s}$. Also apparent from the figures is the reflection of the initial sea surface signal from the offshore boundary and its return across the shelf with a phase speed equal to $-\sqrt{g h}$. The number and relative strength of the reflections vary among the cases according to the strength of frictional damping.

In figures 3-4 and 3-5, the eventual set-down is indicated by the presence of negative contours for large times. Figure 3-3, on the other hand, shows only positive contours indicating that the sea surface never falls below its initial level. The value of the steady-state surface slope can be roughly deduced from the figures by measuring the distance between contour lines at the rightmost edge of each plot. This gives a steady-state surface slope of $5.5 \times 10^{-6}$ for the shallow run and set-down surface slopes of $-1.8 \times 10^{-6}$ and $-1.3 \times 10^{-6}$ for the intermediate and deep runs. The latter two are not yet at steady-state, but longer model runs show that their surface slopes are.

An equivalent and more dramatic way of viewing the evolution of the free surface is shown in figures 3-6-3-8. It clearly shows both the steady-state behavior and the reverberations. The latter shows up as the characteristic zig-zag pattern (also seen in figures 3-3-3-5). Even though the vertical scale in figure 3-6 is different from that of figures $3-7$ and $3-8$, it is easy to tell that 
the shallow case has the largest response. In addition, it has the fastest overall spin-up and its reverbarations die out the fastest. Both of these are evidence of the larger frictional influence in the shallow case.

The wavelike structure in the signal is more obvious in figures 3-6-3-8 than in the contour plots. For example, in the shallow case, there is a smaller wave propagating out of phase with the one originating at the coast. This second wave originates at the shelf break and travels onshore at first. It is not visible in the contour plots, but is seen clearly in figure 3-6 as a second set of zig-zags. The wave is created by a small flux divergence due to application of the clamped boundary condition at the shelf break. There are other wavelike structures propagating in all of the runs. They are responsible for some of the additional variability between the onshore and offshore points in each subplot of figure 3-2.

Figure 3-9 shows time series of cross-shore depth-averaged velocity for the three different wind stresses. Like figure 3-2, there is a significant difference in transient behavior among the subplots. Unlike figure 3-2, however, there is no difference in steady-state. In all cases, the cross-shore velocity is initially onshore as the water moves downwind. It remains almost entirely positive in the shallow case as the sea surface sets up. The intermediate and deep cases show a large offshore flow shortly after the initial set-up. Oscillation between onshore and offshore flow continues, particularly in the deep case. The oscillations are damped, however, so there is a net offshore flow creating a set-down in each case.

All of the runs must have a steady-state cross-shore velocity equal to 0 in order to satisfy mass conservation. This is shown in (2.3) which, with no alongshore variation, reduces to $\partial V / \partial y=0$ at steady-state. Since $V$ is always 0 at the coast, it must be 0 everywhere.

The alongshore depth-averaged velocity signal is shown in figure 3-10. As expected, there is significant variability among the cases. The relative spin-up times and oscillation decay times show the same qualitative behavior as the surface height and cross-shore velocity. At steady-state, the shallow case predictably has the largest alongshore velocity, followed by the intermediate and deep cases.

Taken together, the sea surface and depth-averaged velocity behavior indicates some fundamental properties which have implications for this thesis. They are:

- Many of the differences among the cases are due to differences in the relative importance 

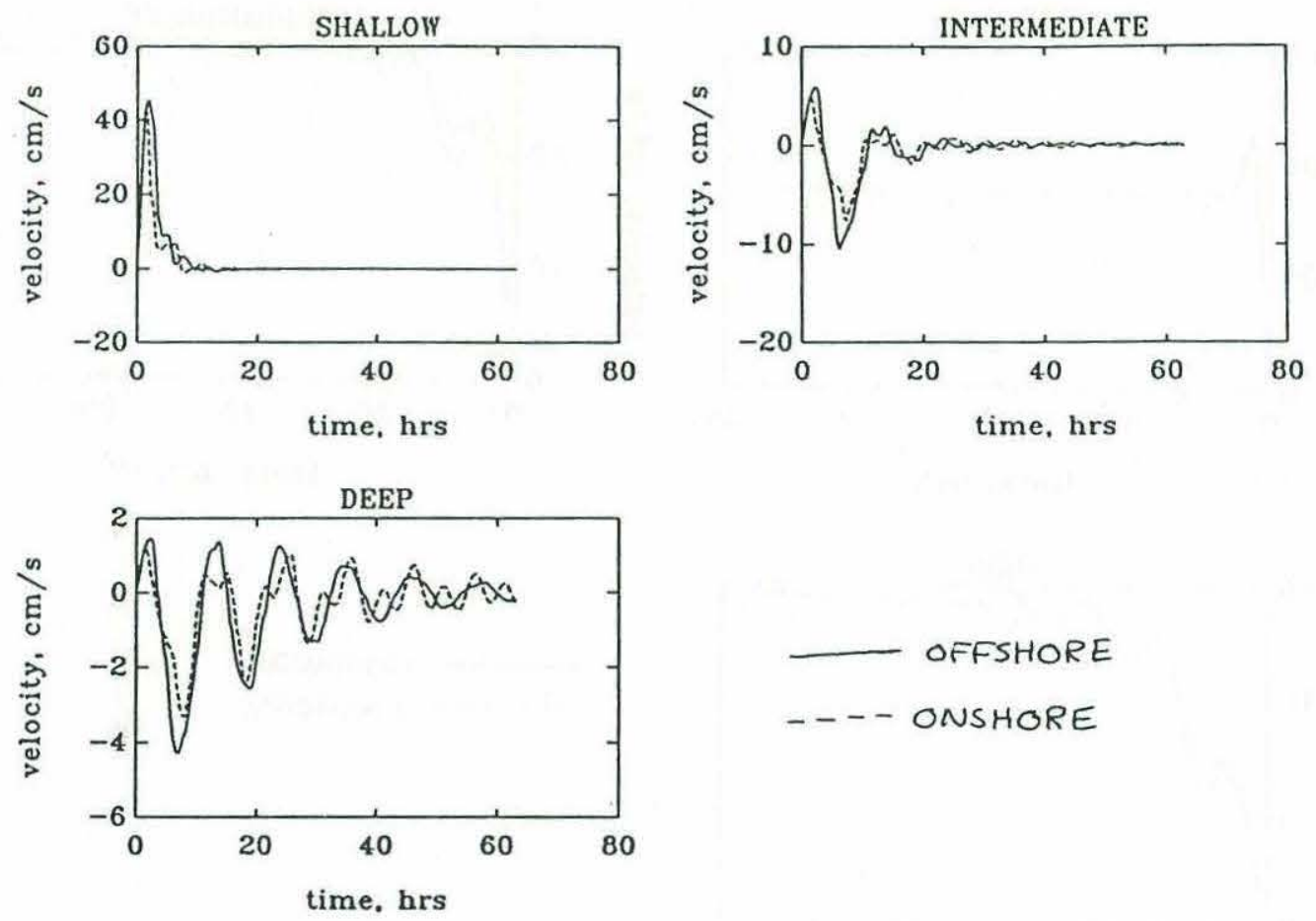

Figure 3-9: Time series of cross-shore depth-averaged veloctiy at two different points across the shelf. The point labelled "inshore" is $75 \mathrm{~km}(7.5 \Delta x)$ from the shore and the point labelled "offshore" is $135 \mathrm{~km}(13.5 \Delta x)$ from the shore. 

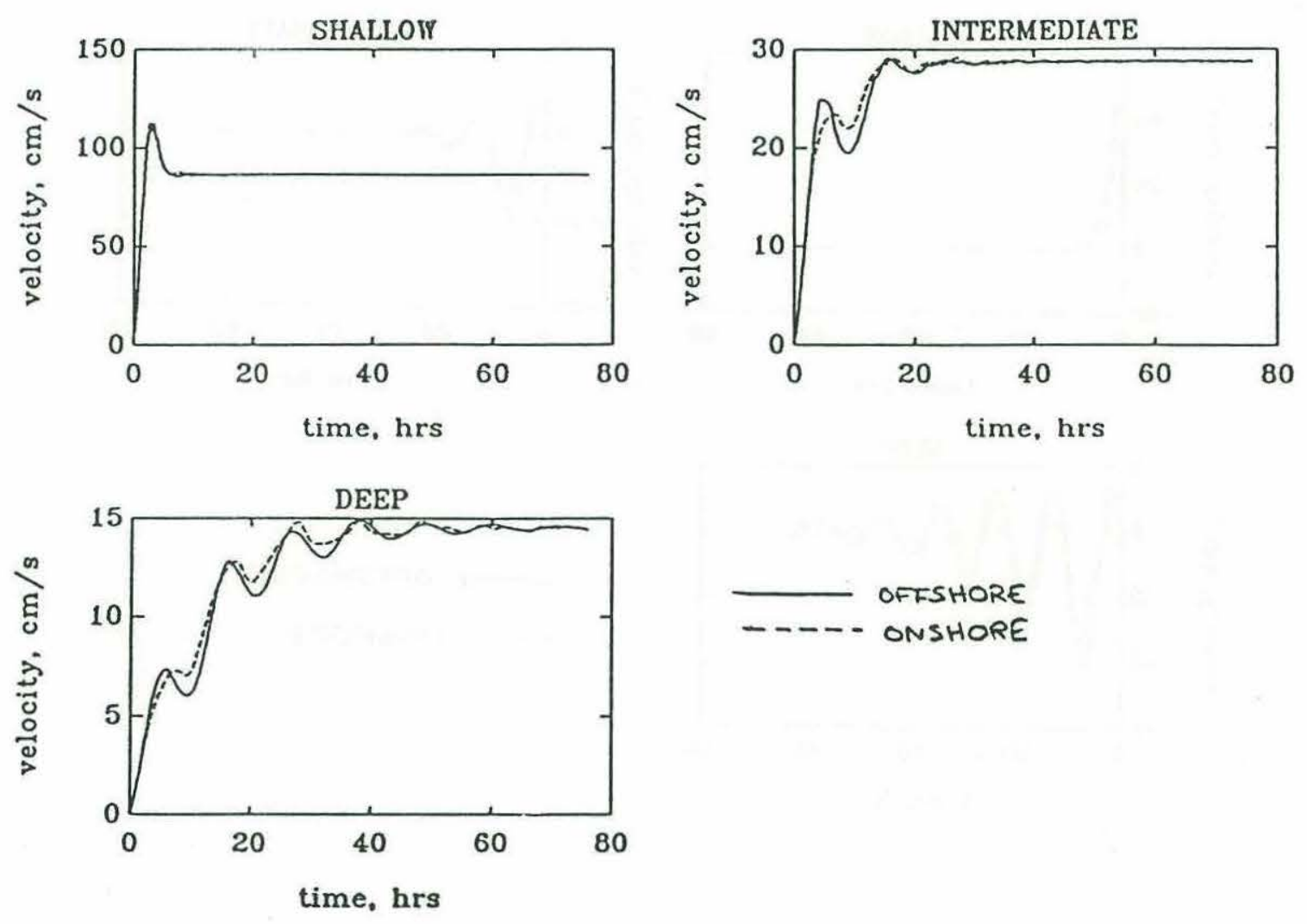

Figure 3-10: Time series of alongshore depth-averaged veloctiy at two different points across the shelf. The point labelled "inshore" is $75 \mathrm{~km}(7.5 \Delta x)$ from the shore and the point labelled "offshore" is $135 \mathrm{~km}(13.5 \Delta x)$ from the shore. 
of bottom stress. This is supported by the fact that the differences in transient behavior primarily consist of variations in spin-up time and damping of the oscillations, both of which are frictional in nature.

- The magnitude of $u_{* s} / f h$ is a significant factor in determining the importance of bottom stress in wind-driven dynamics near an open coast because the only difference among the cases is their assumed values of $u_{* s} / f h$. The results of Jenter and Madsen (1989) also support this idea.

\subsubsection{Momentum balances}

The assertions are confirmed by further examining the dynamics of the shallow, intermediate and deep cases. This subsection contains detailed descriptions of the momentum balances for each. Only results for the offshore point are presented because there is no qualitative difference among locations across the shelf.

In all three cases (figures $3-11 \mathrm{a}, \mathrm{b} ; 3-12 \mathrm{a}, \mathrm{b}$ and $3-13 \mathrm{a}, \mathrm{b}$ ), the very early momentum balance is for a forced inertial oscillation. The first time step requires the local time derivative to balance the wind stress ( As a downwind flow develops, the Coriolis term enters the equation. Pure inertial oscillations last only until the bottom stress first gets updated. This happens at $t \approx 40 \mathrm{~min}, t \approx 1 \mathrm{hr}$ and $t \approx 2$ hrs in the shallow, intermediate and deep cases, respectively.

As the bottom stress develops, the degree to which the inertial oscillations are affected varies. Frequencies and decay scales become a function of shelf geometry and strength of bottom friction (e.g. Madsen, in preparation). In the shallow case, the oscillations are strongly damped (figures $3-11 \mathrm{a}, \mathrm{b}$ ) while in the deep case they persist for a relatively long time (figures $3.13 \mathrm{a}, \mathrm{b})$, but with a frequency shift.

In all three cases, there is a jagged quality to the alongshore bottom stress and acceleration signals. Jumps occur each time the bottom stress is updated, but are more pronounced for smaller $u_{* s} / f h$. Particularly in the intermediate case, the jumps seem to straddle the basic signal, alternating each time the bottom stress is updated. This is not an indication of a poorly resolved physical process, but rather the expression of small numerical waves produced and reinforced each time the bottom stress is updated. These waves also appear in the cross-shore 

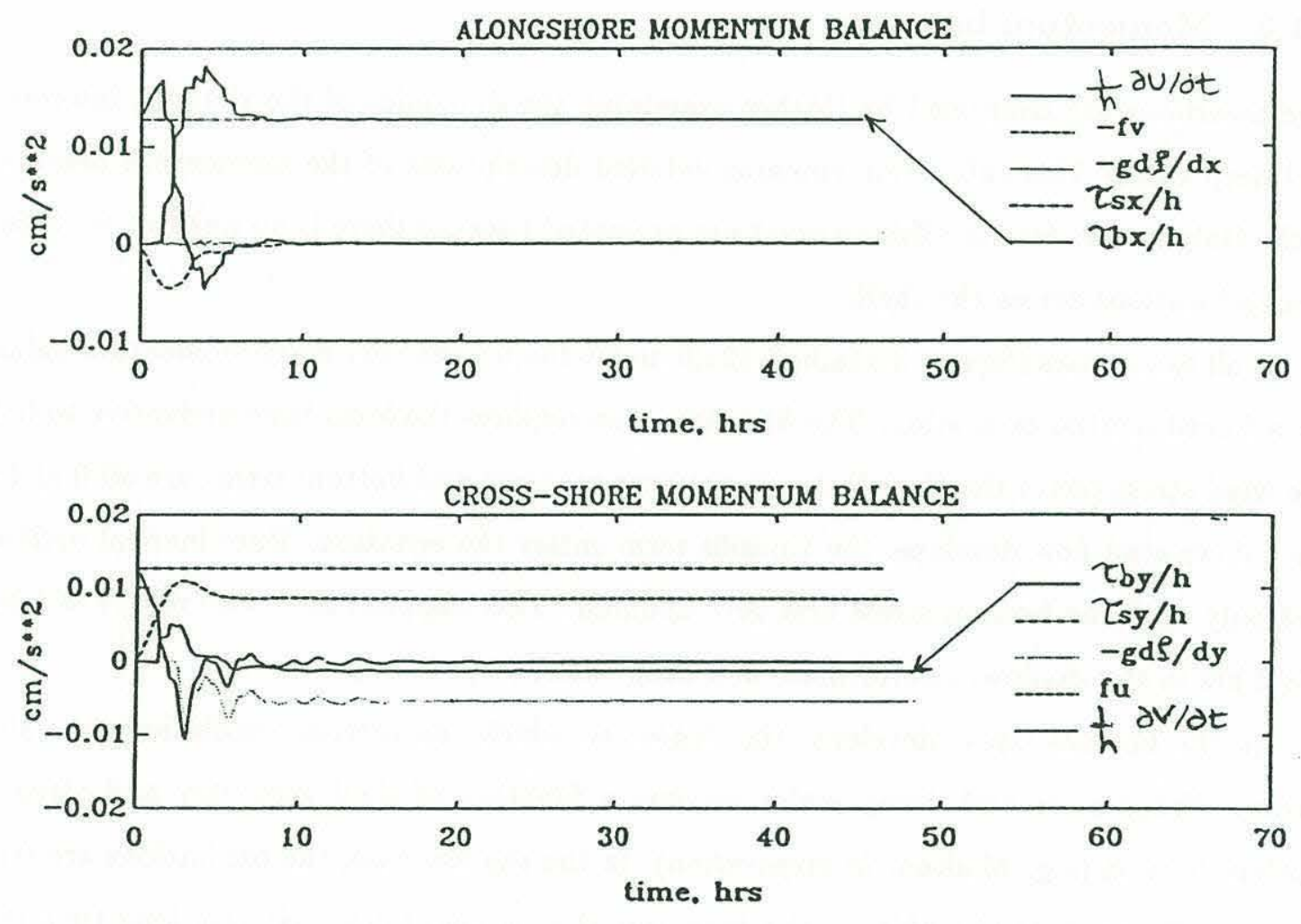

Figure 3-11: Time series of shallow case momentum balance components at the offshore point. 

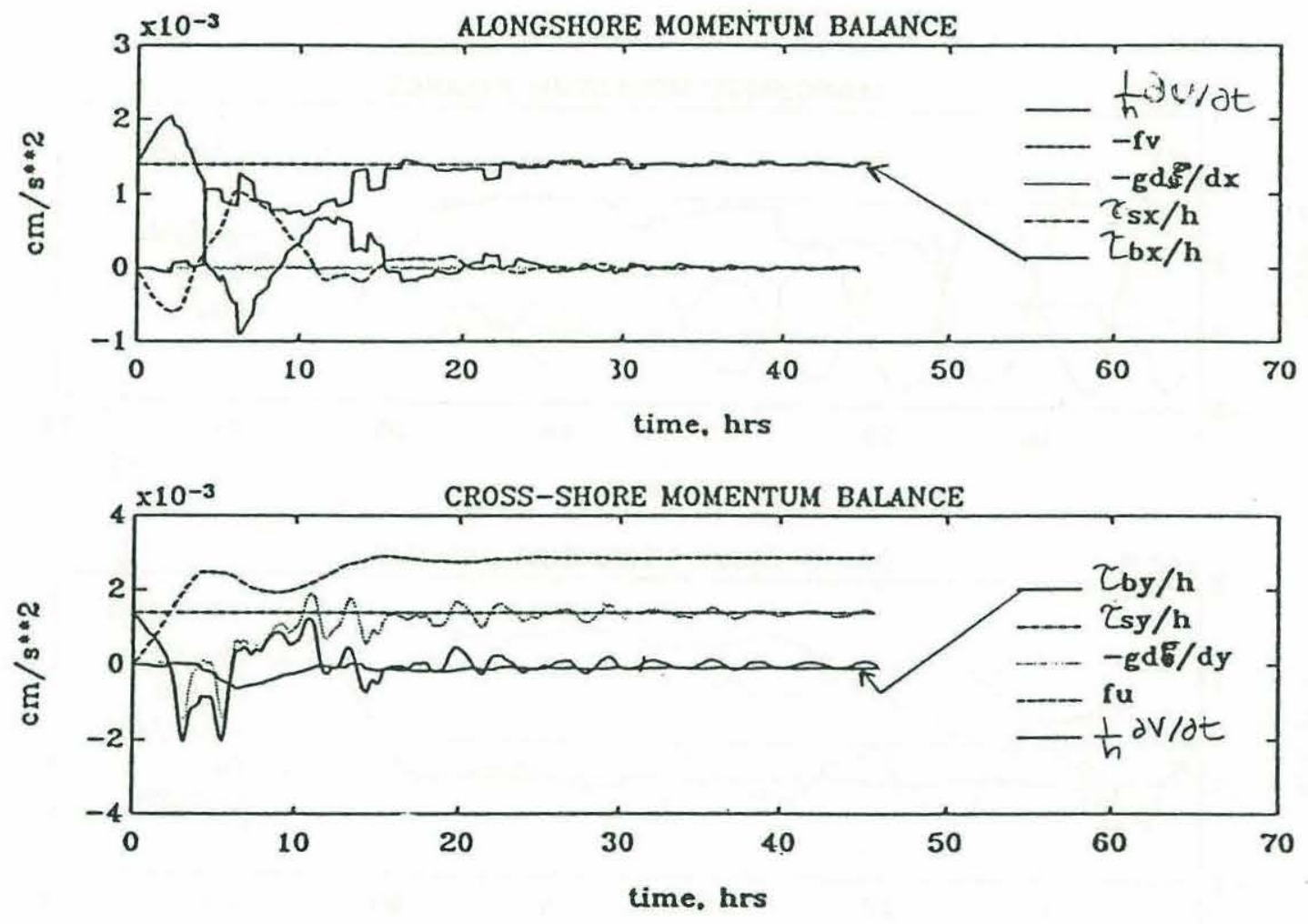

Figure 3-12: Time series of intermediate case momentum balance components at the offshore point. 

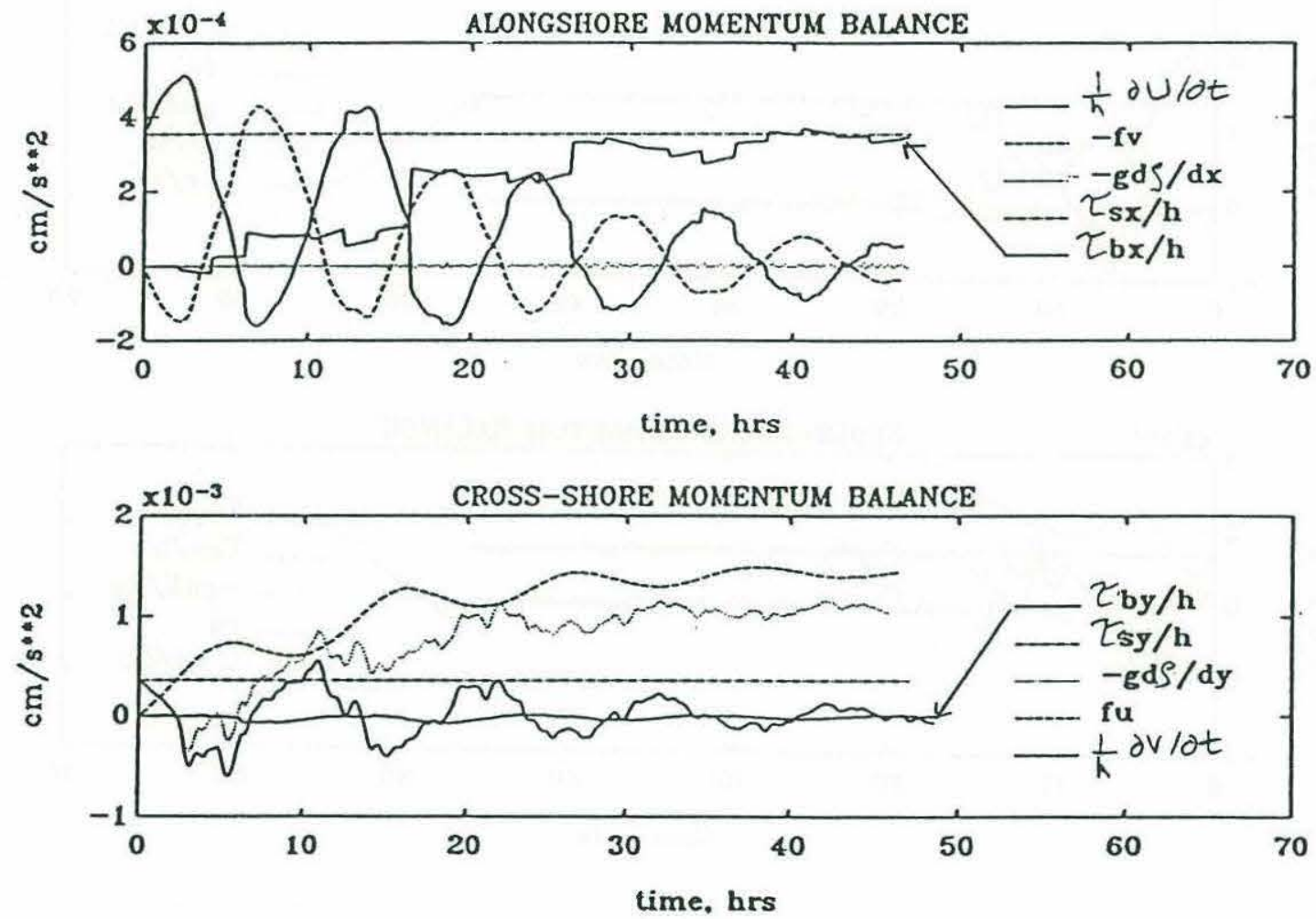

Figure 3-13: Time series of deep case momentum balance components at the offshore point. 
momentum balance in the pressure gradient and local time derivative terms. Clearly, the choice of update period has a strong influence on their nature. This emphasizes the fact that the update time scale must be separate from, and much shorter than, the physical time scales of interest in order for the $2 \frac{1}{2}$-D model to be appropriate. Care must be taken in each application of the model to determine the time scales of interest and to choose the update frequency accordingly, withing the constraints created by approximating the local time derivatives in the DRM.

The alongshore component of bottom stress in all cases grows until it completely balances the alongshore wind stress (figures 3-11a, 3-12a and 3-13a). This means the steady-state alongshore flow is in Couette balance. It is easy to understand this by examining (2.1). The only terms left at steady-state are the bottom and surface stresses. Because of the uniformity assumed here, the alongshore pressure gradient is always zero. Also, the Coriolis term must go to zero as $V$ goes to zero. Thus, the alongshore bottom stress plays a major role in determining the alongshore steady-state flow.

The relative importance of cross-shore bottom stress in determining the cross-shore steadystate flow is variable. Unlike the alongshore bottom stress, it is not constrained by the lack of other forces to balance the wind stress. Therefore, its role is dependent on the strength of the other terms. From figures $3-11 b, 3-12 b$ and $3-13 b$, it is clear that the Coriolis term, surface stress, pressure gradient and bottom stress all play a role in the balance.

In the shallow case, the Coriolis term is slightly less than the surface stress and directed offshore. The pressure gradient is directed offshore also, corresponding to the steady-state setup (e.g. see figure 3.1). The bottom stress, though not a major term in the momentum balance is finite and in the same direction as the wind stress. Thus all of the terms contribute to the balance.

It is worthwile to repeat at this time that a non-zero steady-state cross-shore bottom stress cannot be predicted by a DAM using a traditional bottom stress formulation. Therefore, the momentum deficit created by ignoring the cross-shore bottom stress would be made up for in a traditional DAM by changing the other terms. Since the alongshore flow is predetermined by the Couette balance, the compensation wouldt be made by altering the pressure gradient. This implies that the neglect of cross-shore bottom stress directly affects the prediction of cross-shore surface slope (and its integral, surface height). A quantification of these differences is presented 
later.

The Coriolis term and pressure gradient play a progressively more important role in the cross-shore momentum balance as the wind gets weaker. Conversely, bottom stress plays a decreasingly important role. This indicates merely that the cross-shore balance is becoming more geostrophic as the wind stress gets weaker.

As the bottom stress decreases in relative importance with lessening wind stress, its neglect in the cross-shore direction requires less adjustment of the cross-shore pressure gradient. Consequently, there is little difference in sea surface height signals between the $2 \frac{1}{2}$ - $\mathrm{D}$ model and a traditional DAM for very weak winds.

\subsubsection{Bottom stress and drag tensor}

Figures 3-14-3-16 show time series of bottom stress for the different values of $u_{* s} / f h$. The continuous lines are from the $2 \frac{1}{2}$ - $\mathrm{D}$ model $^{1}$ and the symbols are estimates produced by the DRM. The horizontal spacing of the symbols and the jaggedness of the continuous curves in each plot indicate the update period for the drag tensor. The reason for the jaggedness is that between updates the drag tensor is held constant. In reality, both the drag tensor and depth-averaged velocity should change continuously. However, since the tensor is constant between updates, only the depth-averaged velocity alters the bottom stress. This is insufficient to account completely for the true bottom stress variation. Therefore, at the next update time, the drag tensor makes an abrupt change and the DAM bottom stress jumps accordingly, as was seen earlier.

Despite the jaggedness, the symbols and continuous lines agree very well. This is important because they are not entirely constrained to do so. Agreement is particularly good near steadystate. The better agreement at steady-state is likely an indication that treatment of time dependence in the DRM is not completely adequate, which is not surprising since the DRM was simplified by assuming depth-independent time derivatives.

Agreement is better in the shallow case than in the other two. This likely indicates that the barotropic time derivatives are a better approximation in shallow water, and as discussed above,

\footnotetext{
${ }^{1}$ Note that the continuous lines are merely the bottom stress curves from figures 3-11-3-13 multiplied by the water depth.
} 


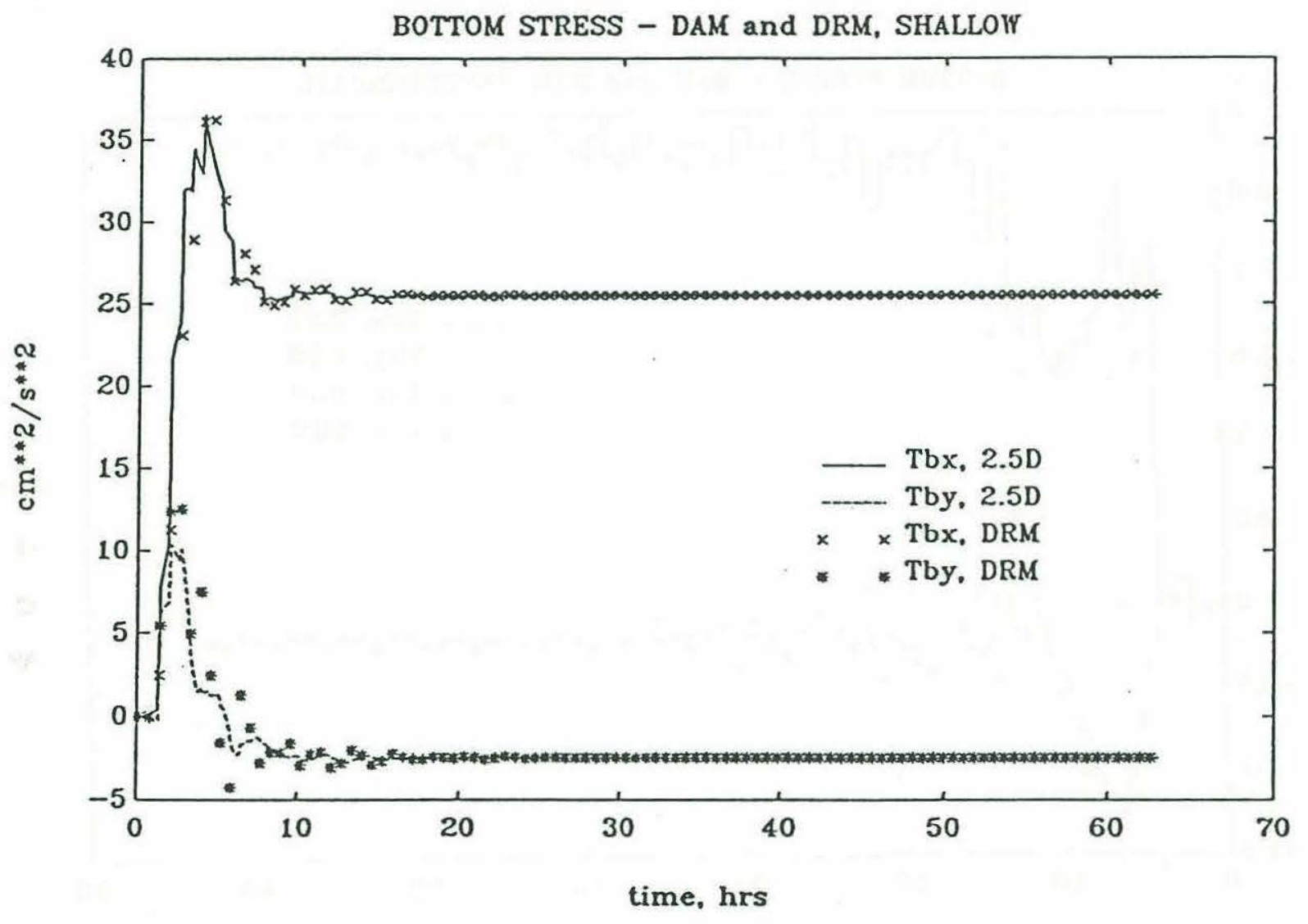

Figure 3-14: Time series of shallow case kinematic bottom stress components $\tau_{b x} / \rho, \tau_{b y} / \rho$ from the DAM (continuous curves) and from the DRM (symbols). 


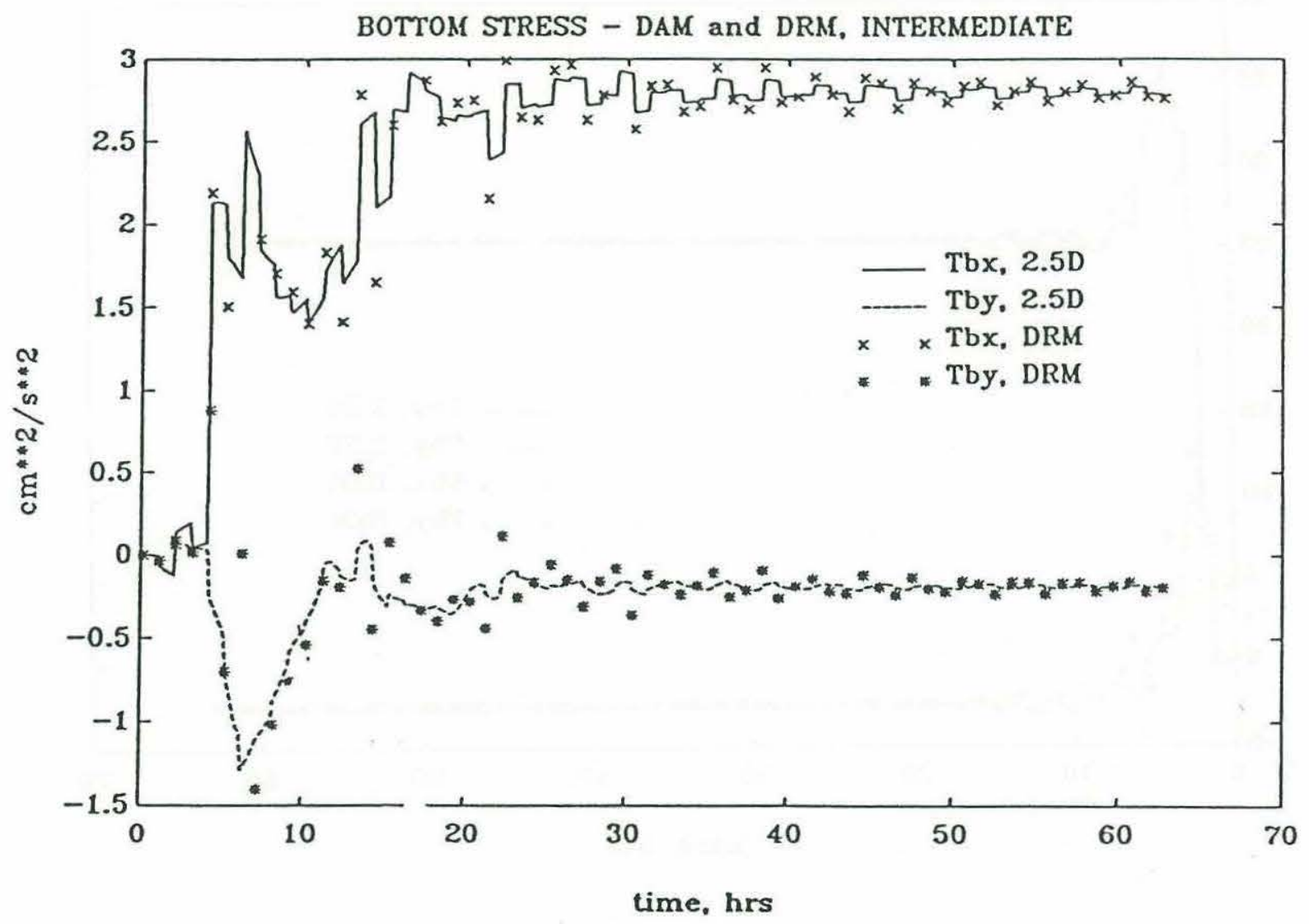

Figure 3-15: Time series of intermediate case kinematic bottom stress components $\tau_{b x} / \rho, \tau_{b y} / \rho$ from the DAM (continuous curves) and from the DRM (symbols). 


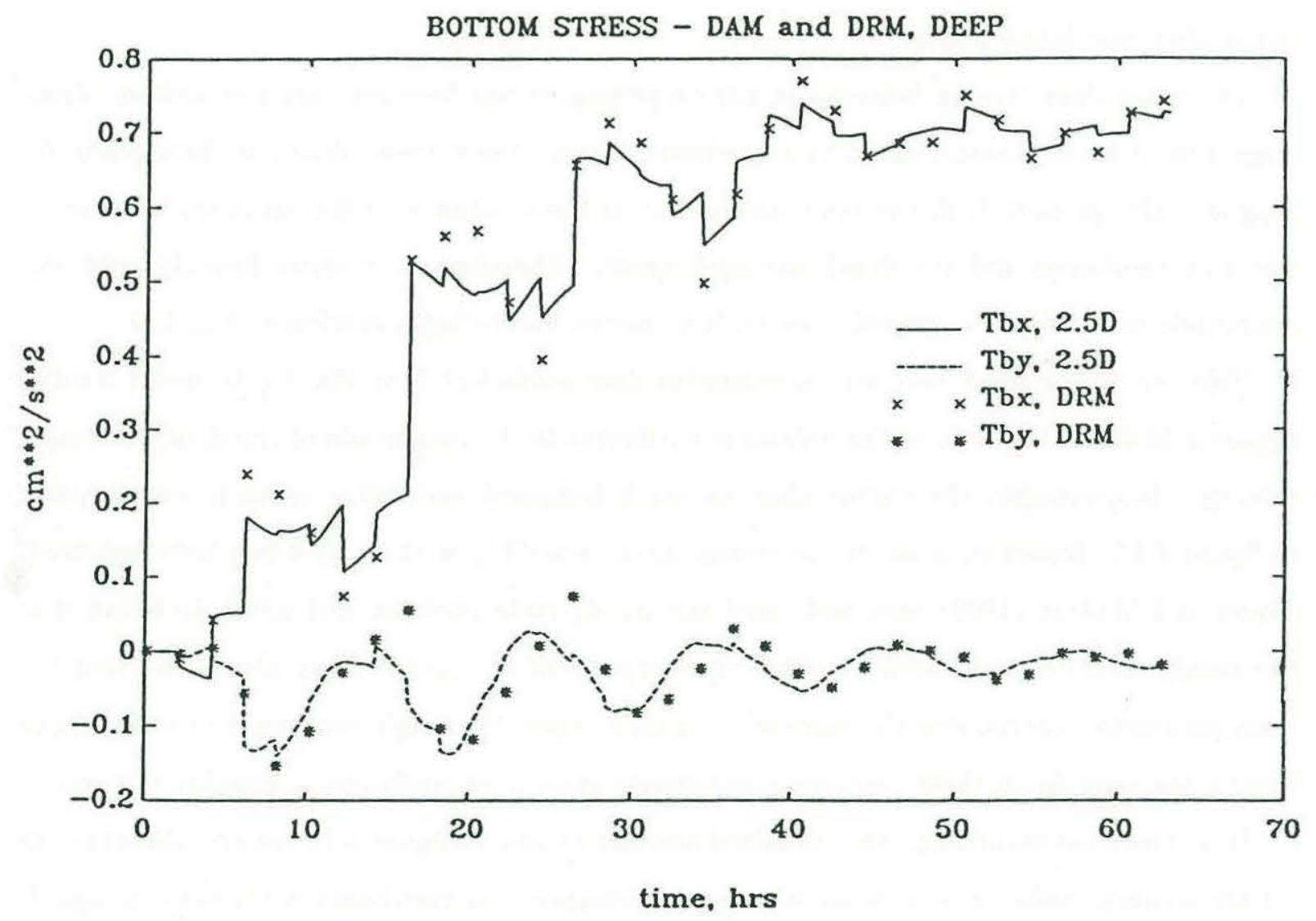

Figure 3-16: Time series of deep case kinematic bottom stress components $\tau_{b x} / \rho, \tau_{b y} / \rho$ from the DAM (continuous curves) and from the DRM (symbols). 
that the length of the update period affects the agreement. The two are clearly related since the approximate treatment of time dependence in the DRM prohibits arbitrarily shortening the update period.

Figure 3-17 shows time series of drag tensor magnitude. It is apparent that the temporal behavior of $r$ is strongly dependent on the strength of the wind stress. In each case, $r$ starts out at zero and grows to its steady-state value over roughly the spin-up time mentioned earlier. Both the shallow and intermediate cases experience an overshoot in the resistance coefficient before reaching steady-state. The steady-state $r$ values are clearly a function of $u_{* s} / f h$ with the shallow case being largest.

The basic time-varying behavior is not surprising, if one believes that the bottom stress magnitude is better approximated by a quadratic, rather than a linear, drag law. In a quadratic drag law, the quantity with the same dimensions as the resistance coefficient is the product of the drag coefficient and the depth-averaged speed. Therefore, if $r$ varies linearly with the magnitude of the depth-averaged velocity, it is merely mimicking a quadratic drag law.

This can be tested by deriving an estimated drag coefficient from the $2 \frac{1}{2}$-D model results. Figure 3-18 shows the ratio of the resistance coefficient to the magnitude of the depth-averaged velocity. Interestingly, the curves show as much temporal variability as their counterparts in figure 3-17. However, most of the steady-state variability with $u_{* s} / f h$ has been removed. Jenter and Madsen (1989) have addressed the steady-state problem and similarly found that the steady-state drag coefficient is relatively independent of $u_{* s} / f h$. They also found that the main parameter determining the value of $C_{d}$ is $z_{0} / h$. Since the roughness length to water depth ratio is the same in all three cases here, the steady-state drag coefficient is roughly the same.

It is somewhat surprising that the initial overshoots seen in figure 3-17 are not absent in the estimated drag coefficient curves since there are corresponding overshoots in the velocity signals (figures 3-9 and 3-10). In fact, the relative overshoot seems to be enhanced for the intermediate and deep cases and unchanged for the shallow case. Apparently in the region of the overshoot, the bottom stress magnitude is increasing faster than the depth-averaged speed squared. Also surprising is the jaggedness of the intermediate and deep case $C_{d}$ signals. Apparently, the small numerically-generated waves superimposed on the physical signal are capable of modulating the drag coefficient. As emphasized throughout this thesis, however, only processes which occur on 


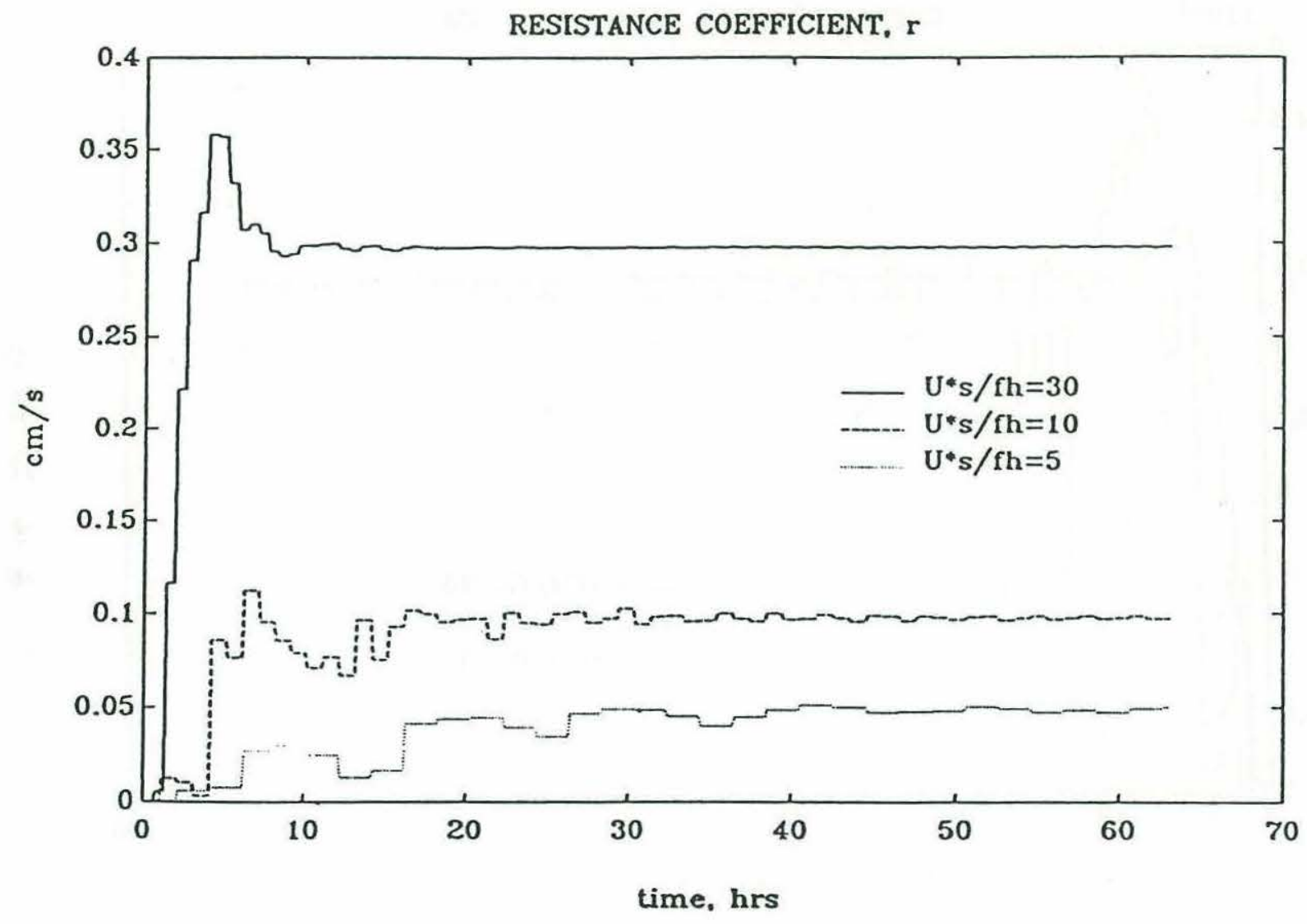

Figure 3-17: Time series of resistance coefficient at the offshore point. 


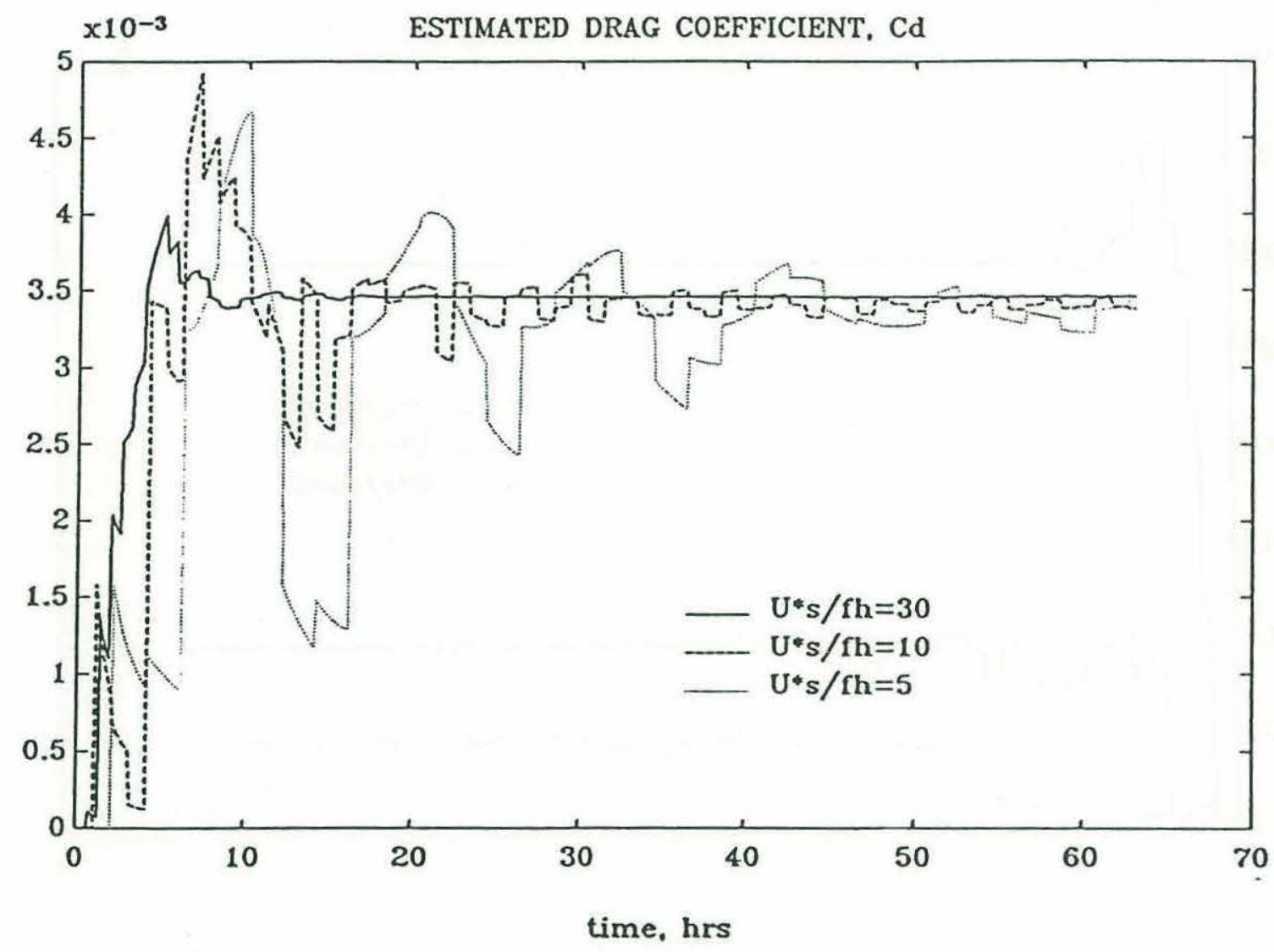

Figure 3-18: Time series of estimated drag coefficient at the offshore point. 
time scales much longer than the update frequency are resolved appropriately. The very high frequency variation is non-physical.

The estimated drag coefficient results imply that the steady-state bottom stress magnitude can be determined fairly accurately by employing a quadratic drag law with a drag coefficient based on a good estimate of $z_{0} / h$. Unfortunately, a correct estimate of bottom stress magnitude is not a correct estimate of bottom stress. The direction of the stress still needs to be determined.

Figures 3-19-3-21 depict the angular information necessary to determine the rotational part of the drag tensor for each of the three wind strengths at early times. The curve labeled "theta" $(\theta)$ is the angular difference between the bottom stress and depth-averaged flow predicted by the DRM. The curve labeled "alpha" $(\alpha)$ is the direction of the bottom stress and the one labeled "beta" $(\beta)$ is the direction of the depth-averaged flow. These angles are defined in figure 2-1.

In each figure, the $\beta$ curve starts out as expected for an inertial oscillation. The direction of flow is linearly proportional to $t$ until the bottom stress is turned on. Rotation continues relatively unperturbed, because bottom stress is initially very small, until the cross-shore pressure gradient arrives. At this time, the frictionally- and geometrically-modulated oscillations commence and the angular variation deviates from linear. The directional oscillations persist for a time commensurate with the level of frictional influence in each case, but eventually die down such that the steady-state direction becomes $0^{\circ}$ or alongshore.

The $\theta$ curve is more difficult to interpret, particularly during the first few update periods. In the shallow case, $\theta$ starts at approximately $-75^{\circ}$, but flips up to $10^{\circ}$ at the second update time. This constitutes a change of roughly $80^{\circ}$ in $40 \mathrm{~min}$. This is not likely physical, but rather a problem induced by the DRM. In fact, it is likely a matter of the initial stresses being so small that their components are poorly constrained by the convergence criteria of the DRM.

After the initial jump, however, the $\theta$ curves behave fairly regularly. In the shallow and intermediate cases, the bottom stress is at first briefly rotated clockwise from the depth-averaged flow. However, it soon passes through the direction of the flow and remains rotated slightly counter-clockwise until steady-state is reached. This means that since the steady-state flow is in the positive $x$ direction, the bottom stress is directed slightly offshore as was indicated in the momentum balances. The deep case bottom stress is always directed clockwise from the depth-averaged flow. 


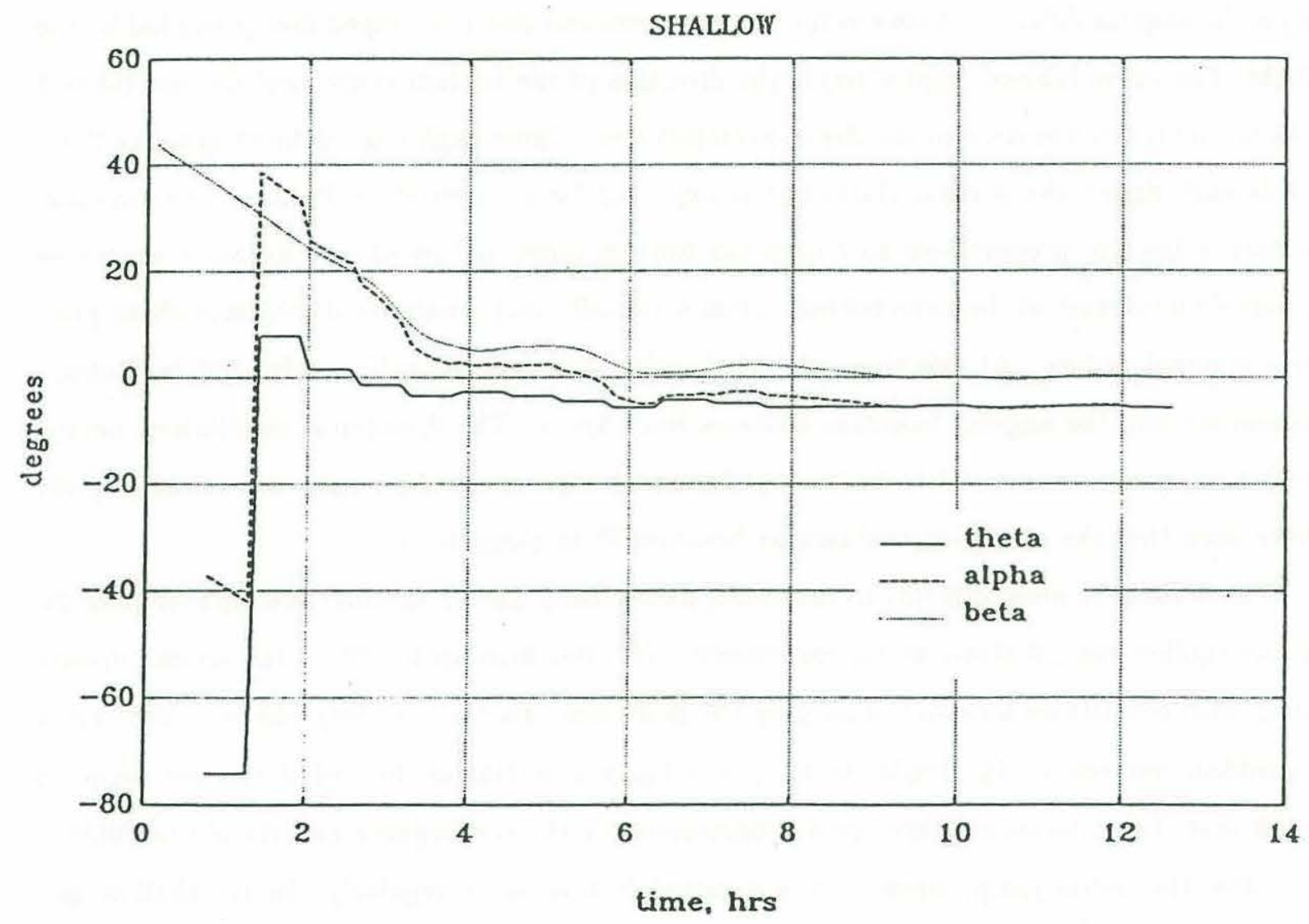

Figure 3-19: Time series of shallow case angular information at the offshore point for early times. 


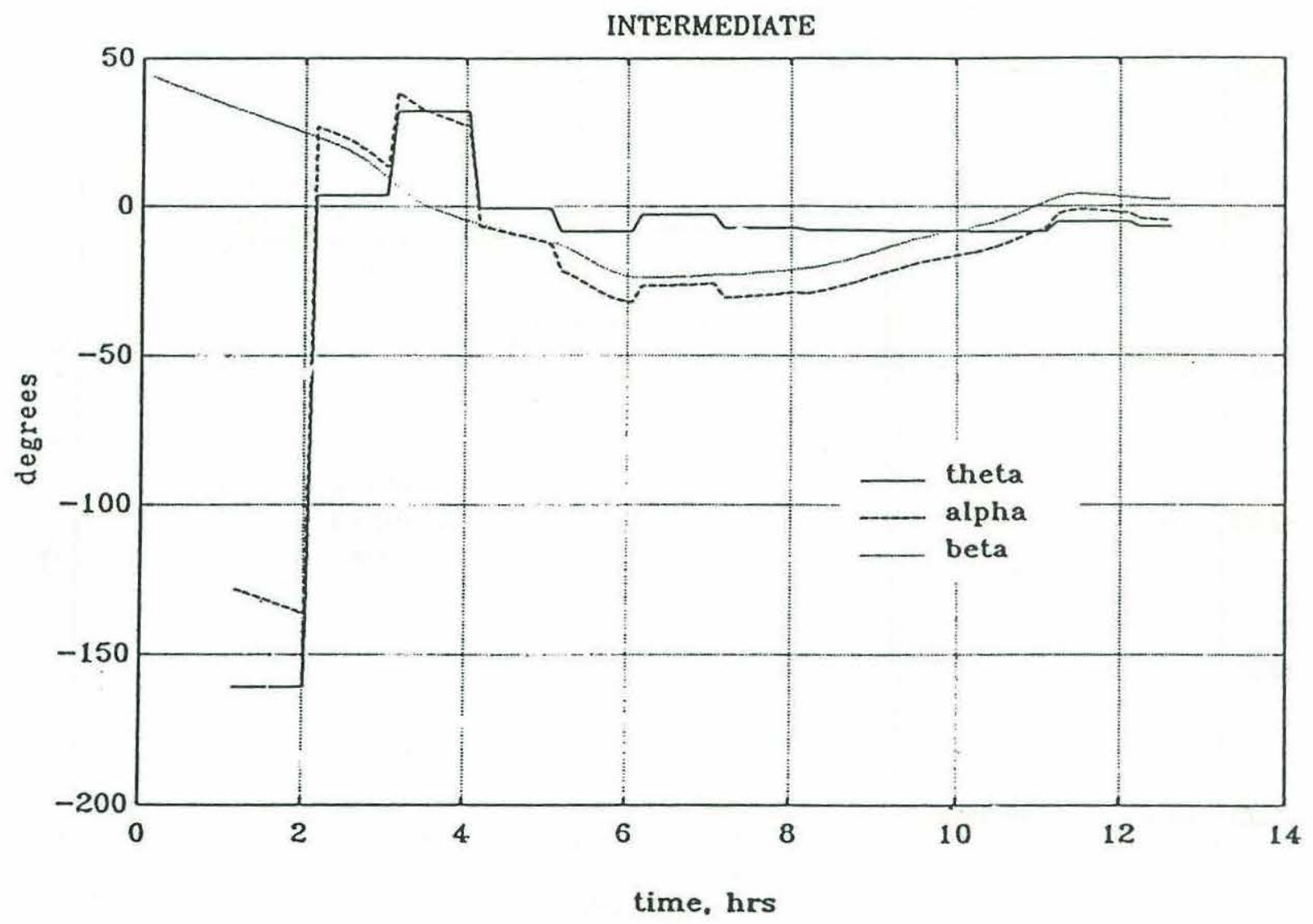

Figure 3-20: Time series of intermediate case angular information at the offshore point for early times. 


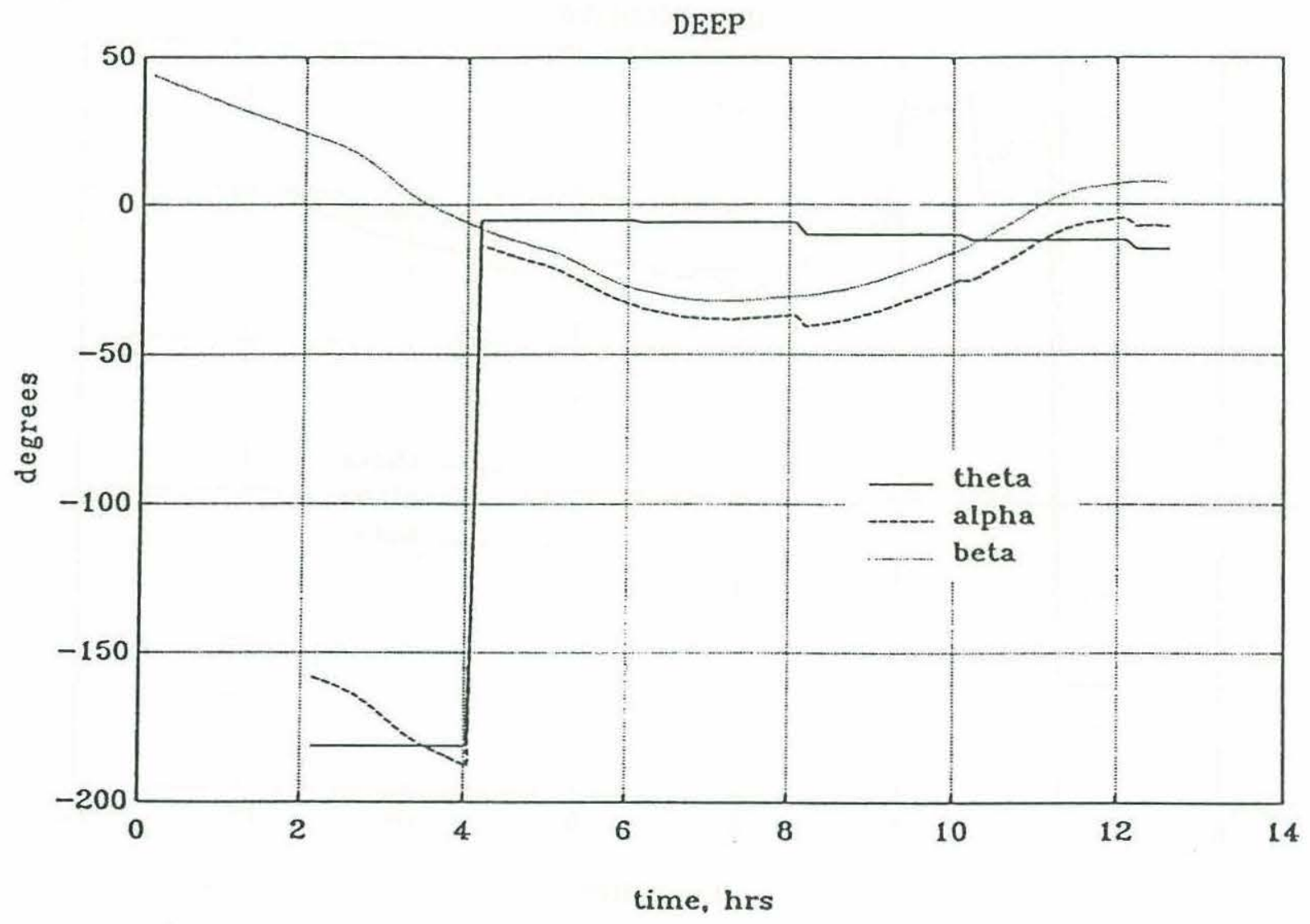

Figure 3-21: Time series of deep case angular information at the offshore point for early times. 
The $\alpha$ curve is just the sum of the $\theta$ and $\beta$ curves. In other words, the direction of the bottom stress in the $2 \frac{1}{2}$-D model is rotated from the direction of the depth-averaged flow by a value equal to the instantaneous value of $\theta$. Since $\theta$ is only updated periodically, the $\alpha$ and $\beta$ curves are always the same distance apart between updates. However, the distance varies each time $\theta$ is updated.

Notice that figures 3-20 and 3-21 do not show steady-state. Only early times are displayed in order to clearly illustrate the initial angular behavior. The angular information reaches steadystate in times comparable to the spin-up times for each case. The steady-state value of $\beta$ is 0 for all the cases since $V=0$. The steady-state values of $\theta$ are $-6^{\circ},-4^{\circ},-1^{\circ}$ for the shallow, intermediate and deep cases, respectively. The progressive decrease in $\theta$ is an indication that the cross-shore bottom stress decreases in relative importance from the shallow to deep cases since $\theta=\alpha=\arctan \left(\tau_{b y} / \tau_{b x}\right)$. Figures 3-11-3-13 support this.

Another thing that the angular results illustrate is that angular differences between the bottom stress and depth-averaged flow are relatively small. Therefore, it seems appropriate to ask "Does including them in a drag tensor really matter?". This question is addressed in the next subsection.

\subsubsection{Differences between traditional and $2 \frac{1}{2}-\mathrm{D}$ model results}

For an initial comparison, a traditional model was run using a resistance coefficient equal to the shallow case steady-state resistance coefficient determined from figure 3-17. Figure 3-22 depicts the surface height variation at the offshore point for this traditional run (dashed line) and for the $2 \frac{1}{2}$-D model (solid line). There is clearly a difference in the two signals. The traditional model spins up more slowly than the $2 \frac{1}{2}$-D model which makes sense in light of the actual time-dependent behavior of $r$ shown in figure 3-17 (reproduced as a subplot in figure 3-22). The traditional model value of $r$, essentially equivalent to $C_{d}|\vec{U}|_{\text {steady-state, }}$ produces friction that is too large until $|\vec{U}| \geq|\vec{U}|_{\text {steady-state. The larger friction also accounts for increased }}$ smoothness in the traditional model results.

A third line (dotted) is also shown in figure 3-22. It corresponds to a traditional model

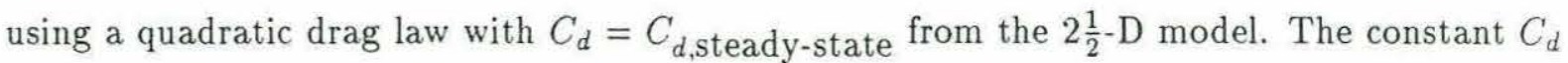
model spins up more slowly than the $2 \frac{1}{2}$-D model and more rapidly than the constant $r$ model, 


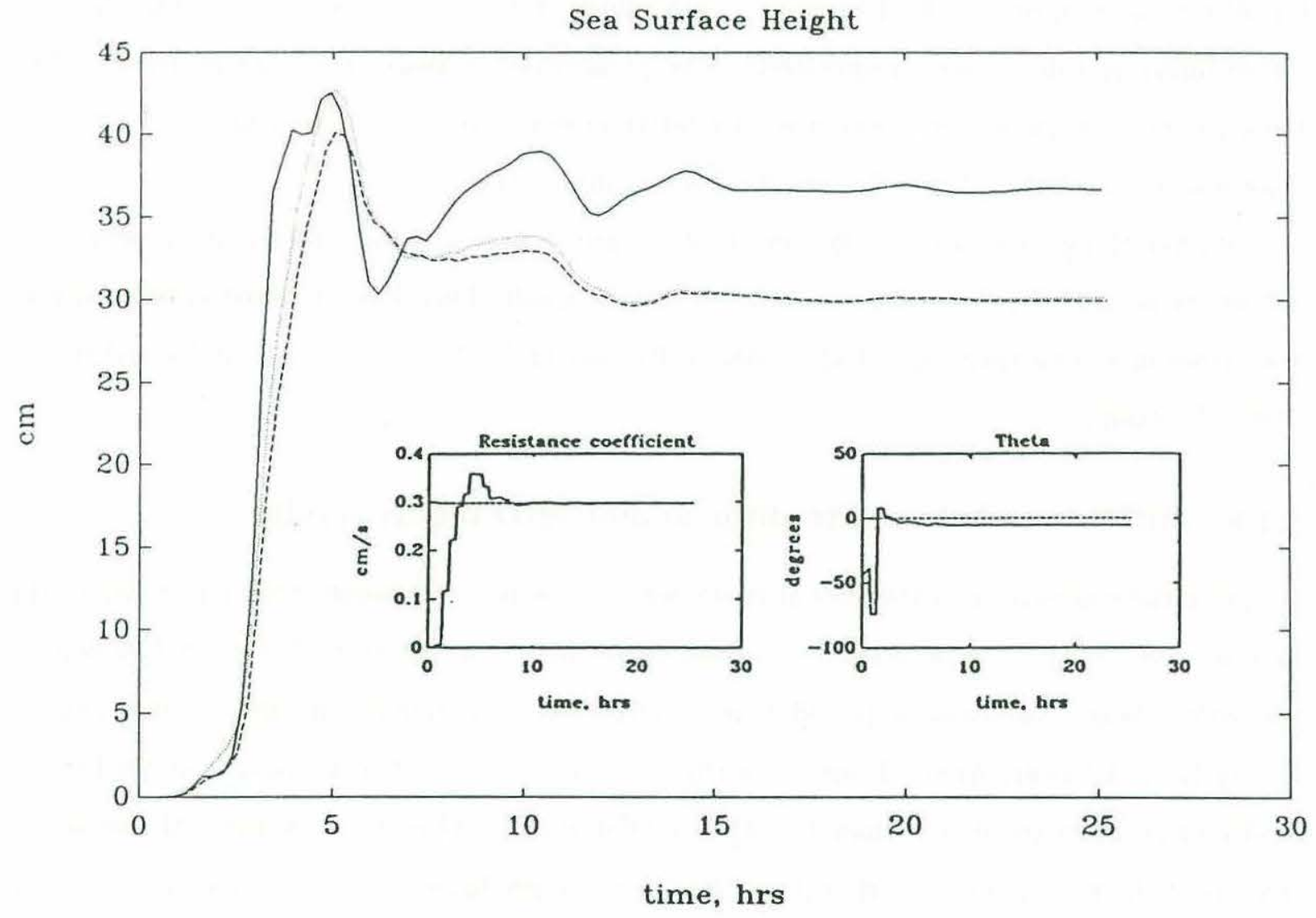

Figure 3-22: Time series of sea surface height at the offshore point for different drag law assumptions: variable drag tensor (solid line), constant resistance coefficient (dashed line), constant drag coefficient (dotted line). 


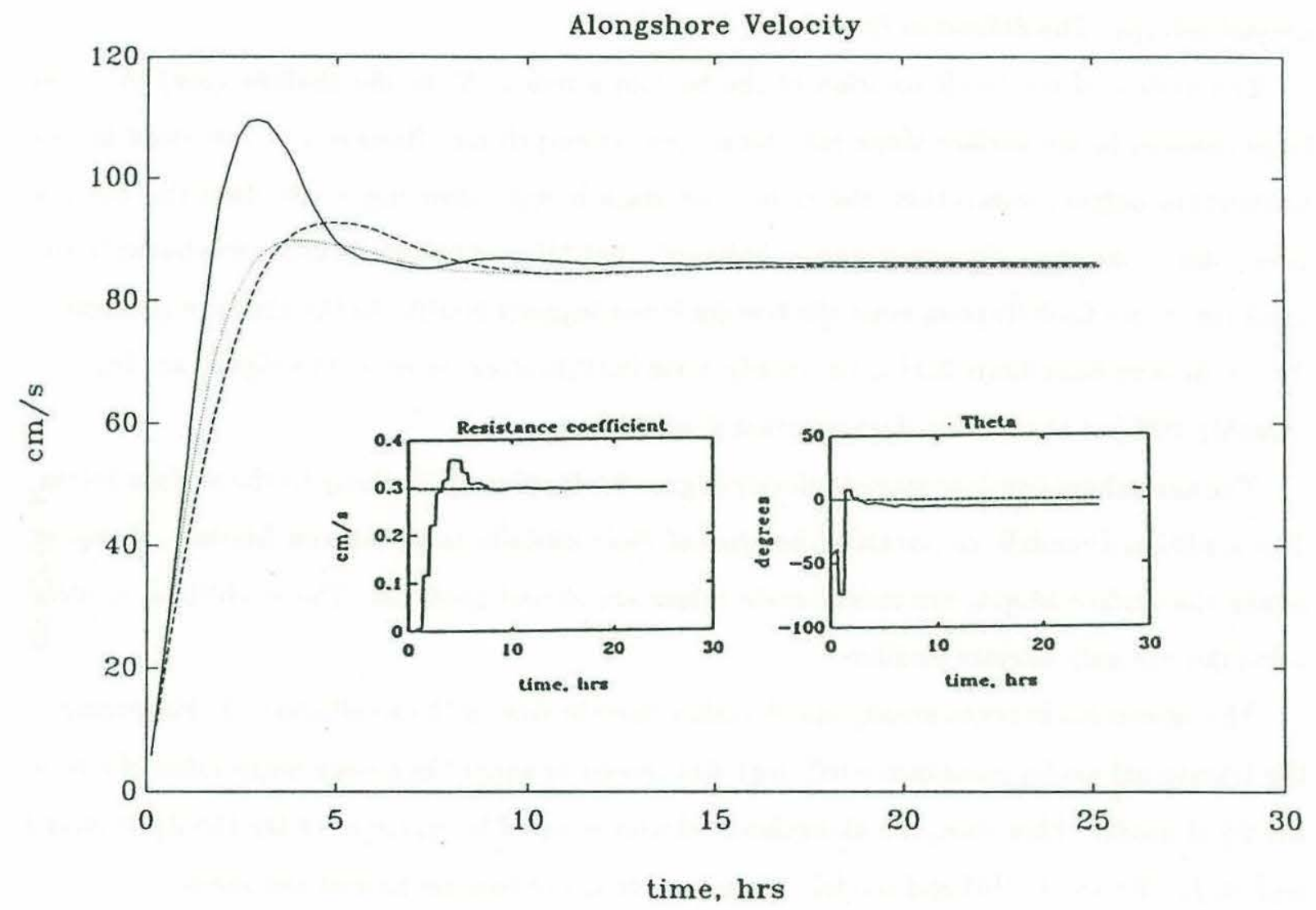

Figure 3-23: Time series of alongshore depth-averaged velocity at the offshore point for different drag law assumptions: variable drag tensor (solid line), constant resistance coefficient (dashed line), constant drag coefficient (dotted line). 
commensurate with the strength of bottom friction at early times.

One of the most important result of this section is illustrated by the difference in steadystate surface heights. Because the steady-state cross-shore pressure gradient is independent of $y$ and the surface is clamped at $y=-L$, the difference between traditional and $2 \frac{1}{2}$ - $\mathrm{D}$ model surface heights at any point is linearly proportional to the distance from the shelf edge. This means the ratio of traditional and $2 \frac{1}{2}$ - $\mathrm{D}$ model surface heights at any point is constant across the shelf. Thus, the ratio of heights at the offshore point can also be interpreted as the ratio of coastal set-ups. The difference from figure $3-22$ is $19 \%$.

The ability of the small rotation of the bottom stress $\left(-6^{\circ}\right.$ in the shallow case) to cause large changes in sea surface slope may be somewhat surprising. However, as discussed in the momentum balance subsection, the critical question is not "How big a role does the bottom stress play in the cross-shore momentum balance?" but "How much of the difference between the wind stress and Coriolis term must the bottom stress support relative to the pressure gradient?" As can be seen from figure $3-11 \mathrm{~b}$, the steady-state bottom stress represents a significant fraction (roughly 20\%) of the steady-state pressure gradient.

The alongshore depth-averaged velocity (figure $3-23$ ) spins up similarly to the surface height. The traditional models are retarded because of their initially large bottom friction. However, unlike the surface height, the steady-state values are almost identical. The traditional models' velocities are only slightly smaller.

The agreement between steady-states makes sense in view of the smallness of $\theta$. For example, the traditional model resistance coefficient was chosen to equal the steady-state value of $r$ from the $2 \frac{1}{2}$ - $\mathrm{D}$ model. Therefore, the alongshore velocity is equal to $\tau_{b x} / \rho r \cos \theta$ for the $2 \frac{1}{2}$ - $\mathrm{D}$ model and $\tau_{b x} / \rho r$ for the traditional model. Since $\cos \theta \approx 1$, the two are almost the same.

The alongshore velocities explain why the difference in steady-state pressure gradient between the traditional and $2 \frac{1}{2}$-D models is nearly equal to the $2 \frac{1}{2}$-D model cross-shore bottom stress. The wind stress is the same for the models, as is the Coriolis term. Therefore, the momentum deficit to be made up by the pressure gradient and bottom stress is the same. Whatever is not balanced by bottom stress must be balanced by the pressure gradient.

Figure 3-24 shows the cross-shore velocity for the traditional and $2 \frac{1}{2}$-D model runs. All go to zero at steady-state, as they must. The transient response mimicks those of the surface 


\begin{tabular}{||c|c|r|c||}
\hline Wind Direction & $r, \mathrm{~cm} / \mathrm{s}$ & $\theta$ & $\frac{\zeta_{\text {constant }, r^{-\zeta} \mathrm{DRM}}}{\zeta_{\mathrm{CRM}}}$ \\
\hline $0^{\circ}$ & .348 & $1^{\circ}$ & .02 \\
$30^{\circ}$ & .325 & $-3^{\circ}$ & -.78 \\
$45^{\circ}$ & .298 & $-6^{\circ}$ & -.19 \\
$60^{\circ}$ & .256 & $-9^{\circ}$ & -.13 \\
$120^{\circ}$ & .261 & $11^{\circ}$ & -.07 \\
$135^{\circ}$ & .301 & $7^{\circ}$ & -.07 \\
$150^{\circ}$ & .328 & $5^{\circ}$ & -.06 \\
$180^{\circ}$ & .348 & $1^{\circ}$ & -.02 \\
\hline
\end{tabular}

Table 3.1: Differences between traditional and $2 \frac{1}{2}$-D model steady-state results for the shallow case.

height and alongshore velocity.

It is useful to examine the difference in surface heights between the models more closely. Figures 3-22-3-24 display results for only one wind direction. Table 3.1 gives ratios of steadystate surface slopes for various wind angles. Notice that the difference between the models is strongly dependent upon wind angle but the order of magnitude seems to be roughly 0.1 . The very large underprediction for winds at $30^{\circ}$ is a result of the sea surface slope being almost zero there. Consequently, the ratio is large because the denominator is small, not because the numerator is large.

There is an asymmetry between shallow cases with angles less than $90^{\circ}$ and those with angles greater than $90^{\circ}$. Obtuse wind angles show smaller differences than their compliments because Coriolis accelerations acts with the wind for angles greater than $90^{\circ}$ requiring larger set-ups. The differences are not due to differences in bottom stresses because bottom stresses have essentially the same magnitude and direction for complimentary wind angles. Therefore, increased denominators in the ratio are responsible for the asymmetry rather than increased absolute differences.

Notice that the bottom stress is almost always directed offshore from the depth-averaged velocity when the wind is between $0^{\circ}$ and $180^{\circ}$. This has important implications for near bottom transport prediction. It means that, whenever the wind has an onshore component, the bottom stress has an offshore component. Traditional DAMs can predict only alongshore steady-state transport. Clearly, $\theta$ cannot be ignored if near bottom transport is of interest. 


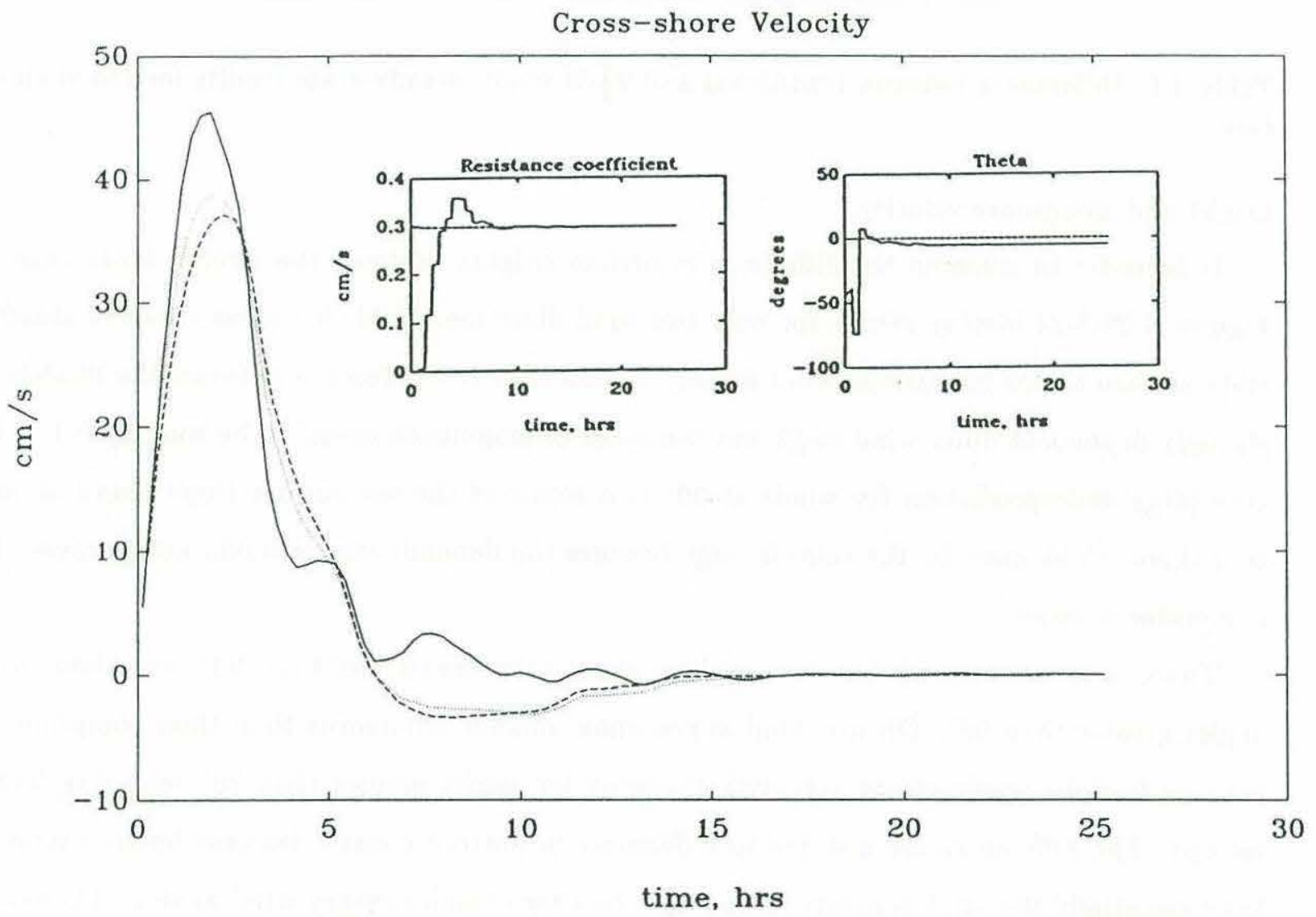

Figure 3-24: Time series of cross-shore depth-averaged velocity at the offshore point for different drag law assumptions: variable drag tensor (solid line), constant resistance coefficient (dashed line), constant dragcoefficient (dotted line). 
Results for angles near $90^{\circ}$ are conspicuously absent from table 3.1 because the alongshore momentum balance in the $2 \frac{1}{2}$-D models is degenerate when the wind is directly onshore, and alongshore uniformity of the domain and wind stress means that there can be no steady-state bottom stress component in the alongshore direction. Since there can be no depth-averaged cross-shore flow, $\theta$ must be $90^{\circ}$. The alongshore velocity can be anything when this is the case. Without constraint on the alongshore flow, it can easily become unstable. In numerical practice, wind-stress angles between $85^{\circ}$ and $95^{\circ}$ tend to have unstable alongshore flows. The instability is, however, not a problem in more realistic cases. If the domain geometry or wind stress has any variability in the cross-wind direction, a pressure gradient will be set-up to balance a cross-wind bottom stress component.

\subsection{Linearly-Varying Depth, Straight Coastline}

Flow over a linearly-varying depth shelf adjacent to a long straight coastline is described here. This represents an increase in complexity from the previous case where there was no crossshore depth variation. Consequently, the two dimensionless parameters which determined the importance of bottom friction in the flat bottom case, $u_{* s} / f h$ and $z_{0} / h$, are now variable in the cross-shore direction. Variation implies that for a single wind stress, this scenario combines a range of behaviors from the previous section.

\subsubsection{Model domain and wind stress field}

The model domain for this scenario is rectangular with the same horizontal dimensions as those in section 3.1 . However, the bathymetry now varies linearly across $70 \%$ of the basin while the outer $30 \%$ is flat. The depth at half a grid space from the coast is $5 \mathrm{~m}$ and increases by $5 \mathrm{~m}$ per grid space (slope $=5 \times 10^{-4}$ since $\Delta x$ is again $10 \mathrm{~km}$ ) until reaching $70 \mathrm{~m}$. The outer shelf depth is constant at $70 \mathrm{~m}$. The strip of flat bathymetry is added offshore in order to have a depth variation similar to one used later in section 3.3. It is important to note that there are no model limitations which say that the depth profile cannot be truncated at the end of its linear variation.

The bottom is again assumed to be covered by roughness elements corresponding to $z_{0}=1$ 
$\mathrm{cm}$ which means that $z_{0} / h$ varies from $2 \times 10^{-3}$ near the coast to $1.4 \times 10^{-4}$ offshore. It was $5 \times 10^{-4}$ in the flat bottom runs. The wind stress is the same as in the flat bottom shallow case. It corresponds to a $33 \mathrm{~m} / \mathrm{s}$ wind speed yielding a range of $u_{* s} / f h$ from 8.6 in deep water to 120 at the coast. The wind direction is $45^{\circ}$. Because the depth is variable, the CFL time step is not strictly applicable. However, a similar criterion is applied. The maximum allowable time step is assumed to be $\Delta t_{\max }=\Delta x / \sqrt{2 g h_{\max }}=270 \mathrm{~s}$. The actual time-step used is $90 \%$ of this value, or $243 \mathrm{~s}$.

The diffusion time scales, as determined by (2.47), range between $315 \mathrm{~s}$ and $4375 \mathrm{~s}$. The update frequency chosen is once every 5 time steps. This means the diffusion process is somewhat under-resolved near the coast (depth shallower than 20m) and over-resolved offshore (depth deeper than $20 \mathrm{~m}$ ).

\subsubsection{Surface height and depth-averaged velocity}

Figure 3-25 shows time series of the basic variables at two cross-shore points. The onshore (offshore) point corresponds to a water depth such that $u_{* s} / f h$ equals the shallow (intermediate) values from section 3.1. Specifically, the onshore point is in $20 \mathrm{~m}$ of water and the offshore point is in $60 \mathrm{~m}$ of water. Notice that although $u_{* s} / f h$ at the offshore point agrees with the intermediate case of section 3.1 , the value of $z_{0} / h$ is smaller.

Figure 3-25a shows that the surface height signal, both onshore and offshore, looks like a combination of the flat bottom shallow and intermediate responses. The signal magnitude is comparable to the flat bottom shallow case, but the spin-up time is more like the flat bottom intermediate case. Reasons for this are given below. The initial response at both points is flat, followed by a time-lagged set-up. The onshore point sets-up first reaching a value comparable to the shallow case in section 3.1. This happens slightly later, however, than the comparable point in the flat bottom case because the wave travelling out from the coast is travelling through shallower water here. Conversely, the offshore point experiences a slightly earlier arrival since the increased phase speed offshore of the $20 \mathrm{~m}$ depth contour allows the signal to more than regain the time lost travelling in shallow water. Note that the signal at the offshore point has a much larger amplitude than that of the intermediate case in section 3.1. This indicates that the amplitude of the transient wind-driven signal is determined at the coast which, makes sense 
because the initial bulge is produced there.

After the short set-up period, the sea surface height at both points drops significantly as the water moves offshore. This creates an overall set-down. Damped oscillations continue superimposed on the set-down for a time comparable to the flat bottom intermediate case decay time, whichis understandable because the waves generated at the coast are not damped as quickly when they propagate through deep water.

Figure 3-26 presents another picture of the spin-up process. The wave progresses outward from the coast in much the same way as the flat bottom case, but the spatially-varying phase speed causes curvature in the contour lines. The phase speed is slower nearshore and faster offshore giving the signature a cusp-like appearance. It is possible to determine an average phase speed based on the total travel time for the wave to cross the shelf using travel time $=$ $\int_{0}^{L}(1 / c) \mathrm{d} y$. For the present domain geometry, this yields a phase speed of $15.25 \mathrm{~m} / \mathrm{s}$. A phase speed estimated using the leftmost $10 \mathrm{~cm}$ surface contour yields an average phase speed somewhere between 14 and $16 \mathrm{~m} / \mathrm{s}$ which is in good agreement with the theoretical value.

The oscillations eventually damp out, and a good approximation to steady-state is reached in roughly $20 \mathrm{hrs}$. Unlike the flat bottom cases, the steady-state surface slope is variable across the shelf. It changes monotonically from negative offshore to positive onshore with the steepest slope found at the coast. Despite the positive slope nearshore, the water level of the entire shelf is below its initial value.

Figure 3-27 shows the juxtaposition of sea surface height "snapshots" every 5 time steps. Notice that the perspective has been chosen so that the steady-state surface profile can be more easily seen. The cusp-like nature of the signal is clearly visible. It is also easy to see the rapid drop in sea surface height and subsequent oscillations after the initial set-up . Higher frequency small scale oscillations, which were not apparent in figures $3-25$ a and 3-26, are seen on the face of the larger signal. Similar motions appeared in the flat bottom cases.

The fact that the sea surface has a minimum in the interior of the domain is apparent as steady-state is approached. Clearly the cross-shore momentum balance varies in $y$ since the cross-shore pressure gradient differs so greatly across the shelf (even changing sign). Remember that this did not happen in the flat bottom case where the steady-state cross-shore pressure gradient was constant everywhere. Further discussion of the pressure gradient is provided in 

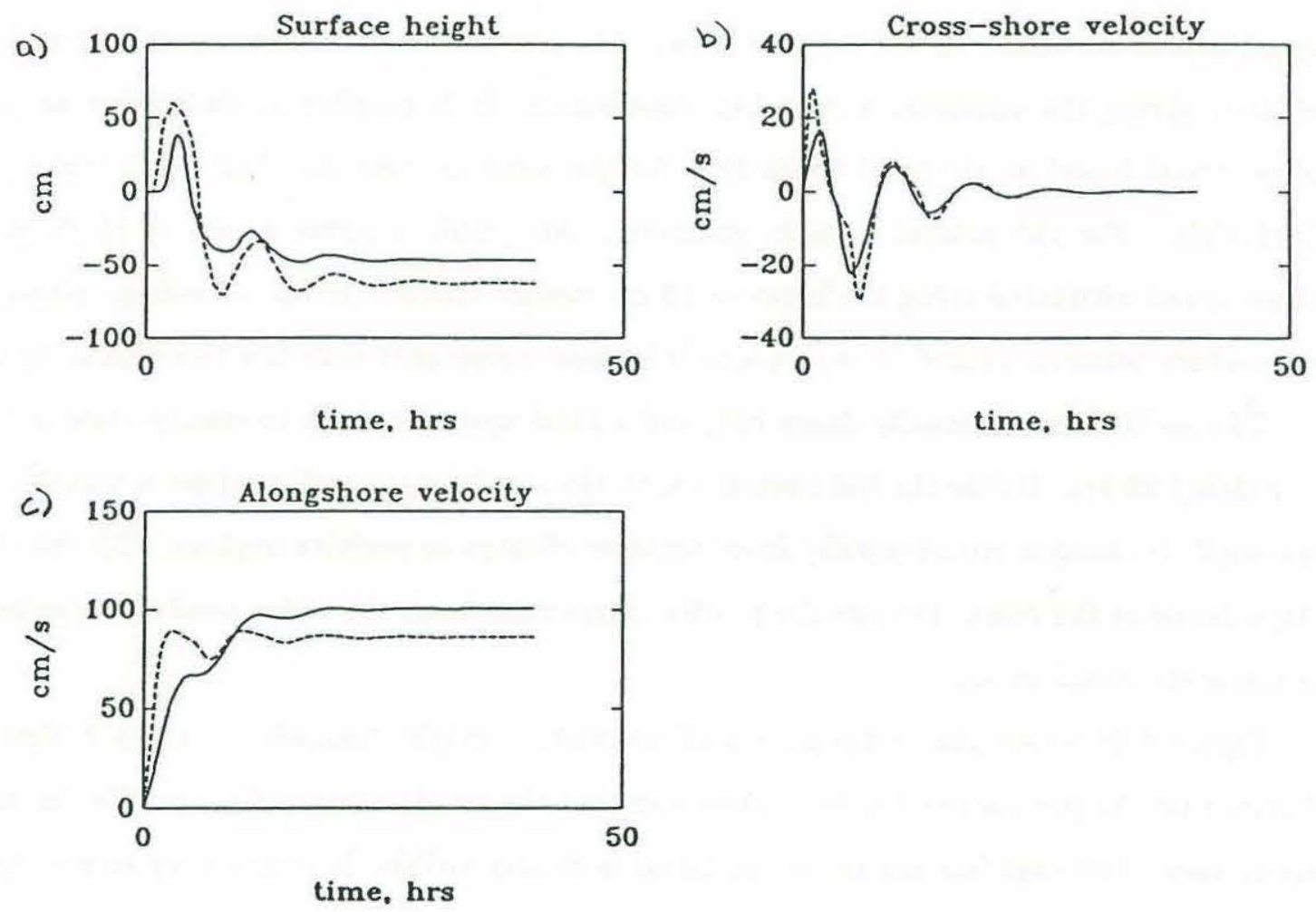

Figure 3-25: Time series of a) sea surface height, b) depth-averaged cross-shore velocity and c) depth-averaged alongshore velocity at two points on the shelf. The offshore point (solid line) is located in $60 \mathrm{~m}$ of water and the onshore point (dashed line) is located in $20 \mathrm{~m}$ of water. 


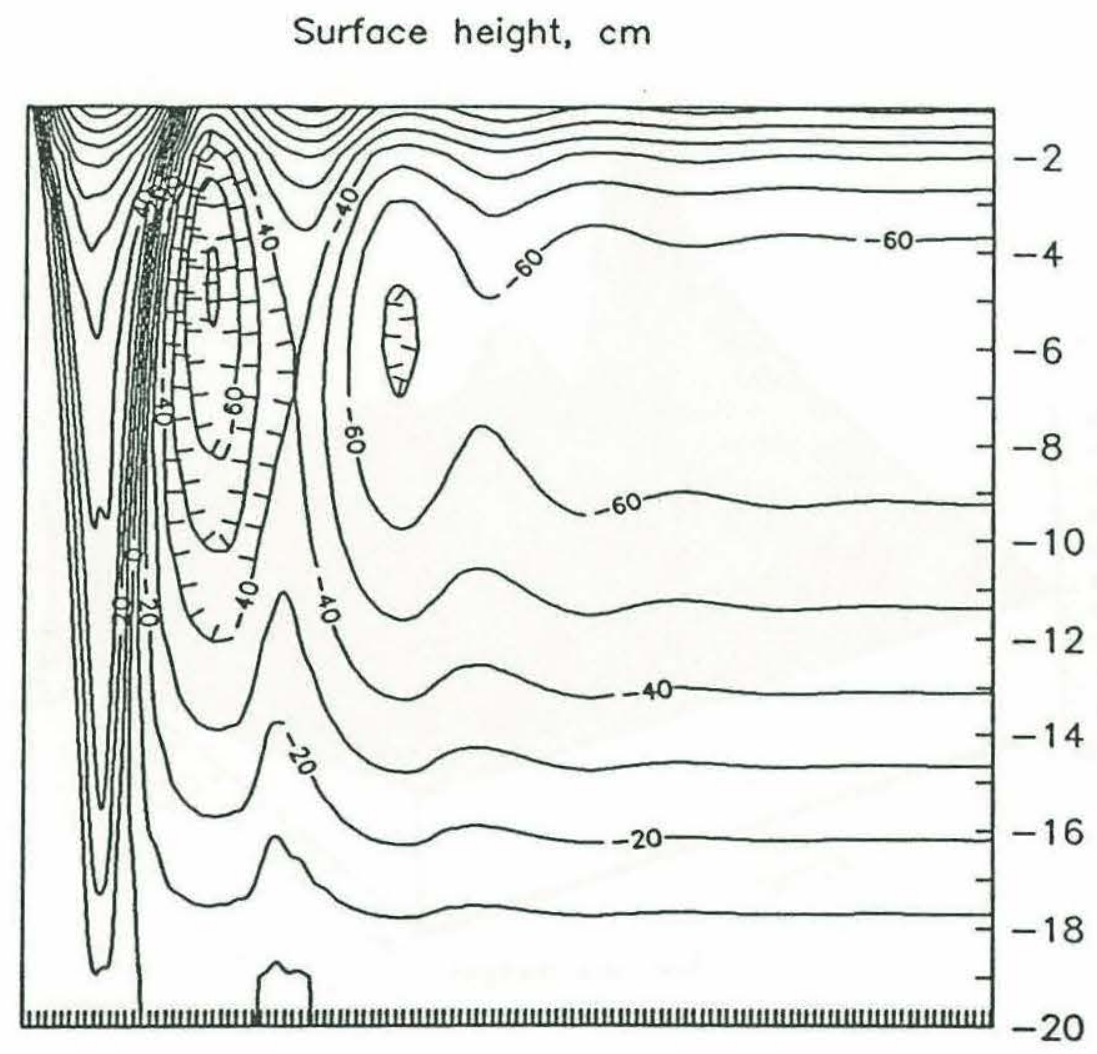

Figure 3-26: Contour plot of sea surface height on the $y$ - $t$ plane. Tic marks are at intervals of $5 \Delta t$ and $\Delta x$ on the horizontal and vertical axes, respectively. The time axis ranges from 0 to 40 hrs. 


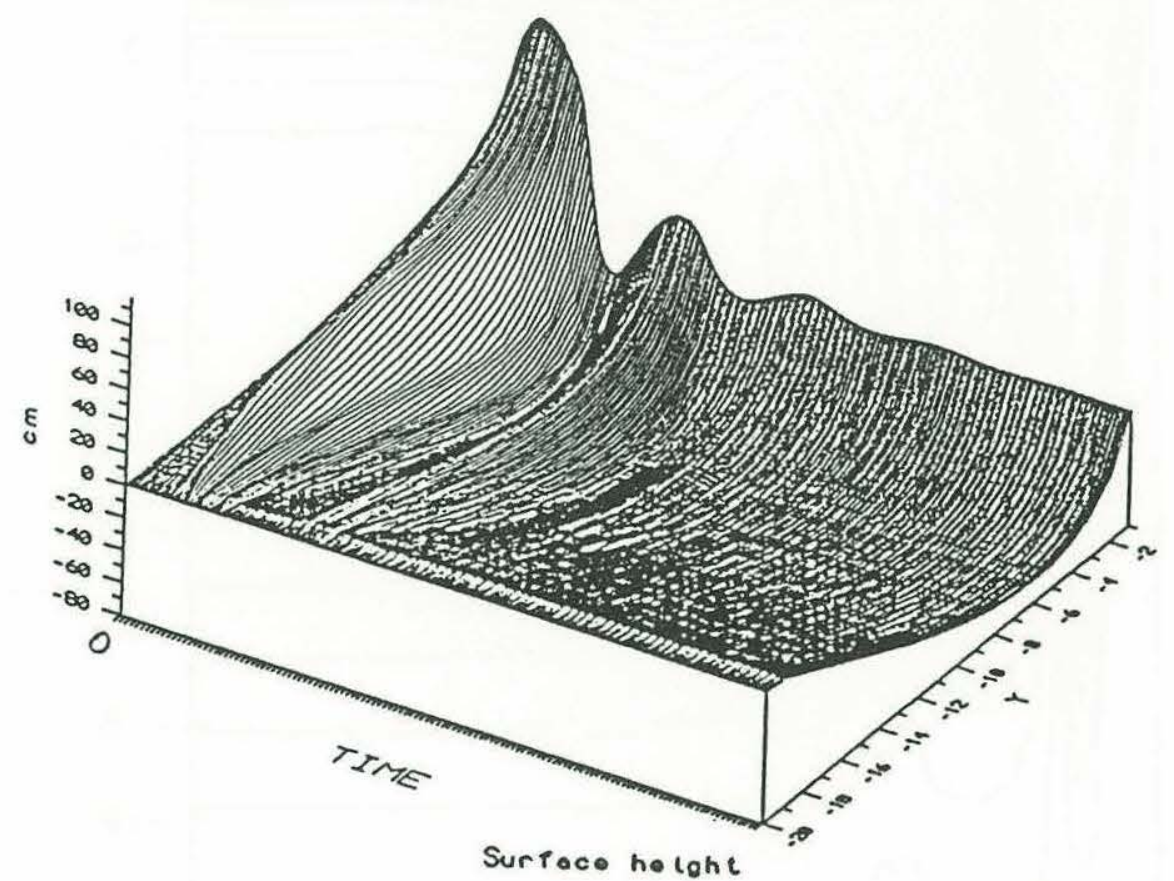

Figure 3-27: 3-D plot of sea surface height in $y$ - $t$ space. Tic marks are at intervals of $5 \Delta t$ and $\Delta x$ on the $t$ and $y$ axes, respectively. The time axis ranges from 0 to $40 \mathrm{hrs}$. 
the momenum balance subsection below.

The cross-shore velocity is shown in figure $3-25 \mathrm{~b}$. As with the surface height, the oscillation magnitudes are comparable to the flat bottom shallow case while the decay time is similar to the flat bottom intermediate case. Initially, the flow accelerates faster at the onshore point. This is understandable since, in the absense of bottom stress and a pressure gradient, the cross-shore momentum balance reduces to $\partial V / \partial t=\tau_{s y} / \rho$ yielding essentially equal transports across the shelf. Equal transports mean larger depth-averaged velocities in shallow water. Each series peaks due to the establishment of an adverse pressure gradient upon the arrival of the sea surface signal. The flow is then rapidly accelerated in the opposite direction and soon turns offshore. The cross-shore flow oscillates a few times before reaching steady-state. Both time series show a net offshore flow (as would be expected everywhere in light of figures 3-26 and 3-27 having only negative sea surface values at steady-state).

Figure 3-25c shows time series of alongshore velocity. Both points spin-up with maximum speeds similar to the shallow flat bottom case. The difference between the flat and linearlysloping bottom cases, however, is that the steady-state alongshore flow varies across the shelf. This is entirely due to the fact that $z_{0} / h$ varies across the shelf causing the DRM to produce a variable frictional relationship across the shelf. Cross-shore variation cannot be produced

by a model with spatially-constant $r$ (or $C_{d}$ ) since the steady-state depth-averaged alongshore momentum balance would not vary across the shelf $\left(\tau_{s x} / \rho=r U / h=r\right.$ xalongshore depthaveraged velocity)).

\subsubsection{Momentum balances}

The addition of cross-shore depth variation in this scenario implies that the momentum balance at different points across the shelf varies. Figures 3-28 and 3-29 show momentum balances at the onshore and offshore points, respectively.

At the onshore point, the alongshore momentum balance (figure 3-28a) initially behaves like a forced inertial oscillation, but only for the short time that the bottom stress is zero. Once the bottom stress is updated, it rapidly grows until essentially equaling the surface stress. This takes slightly less than $5 \mathrm{hrs}$ (a time comparable to the shallow flat bottom spin-up time). The approach to steady-state is subsequently modulated by damped modified inertial oscillations 

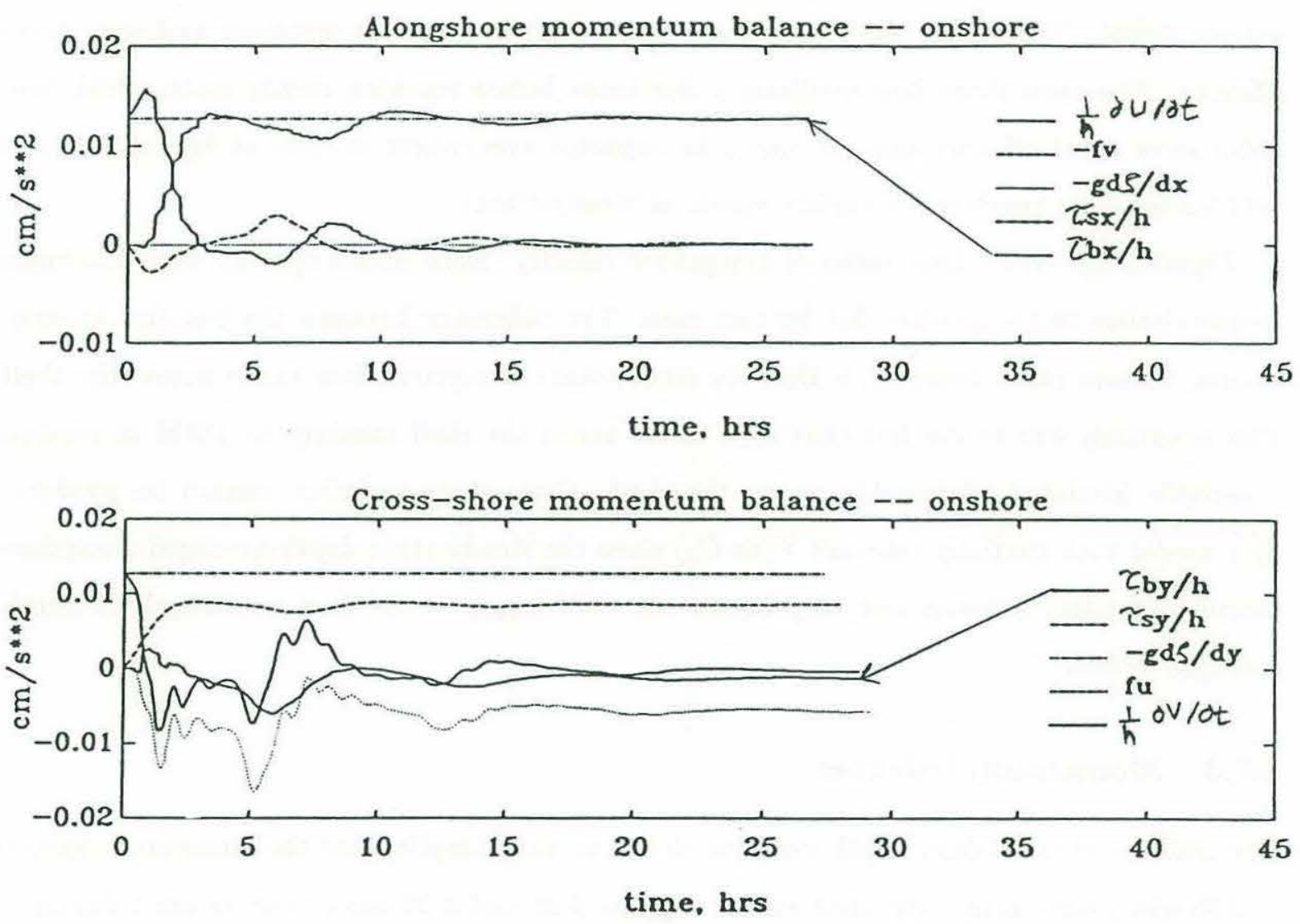

Figure 3-28: Time series of momentum balance components at the onshore point. 

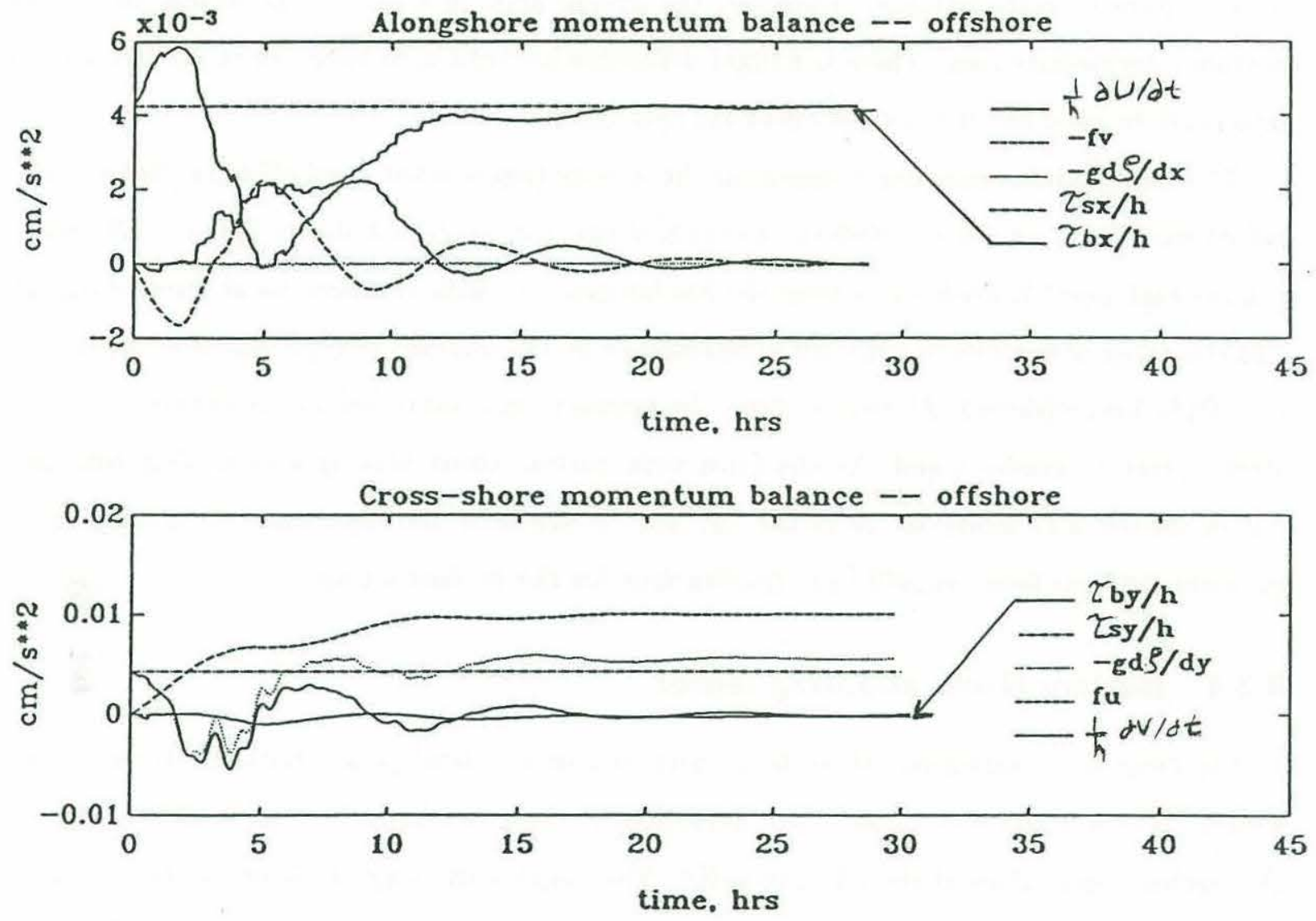

Figure 3-29: Time series of momentum balance components at the offshore point. 
superimposed on the basic Couette balance. The oscillations persist much longer than they do in the flat bottom shallow case indicating that waves are damped more slowly here. This makes sense because the waves spend some time propagating through deep water where friction is weaker.

The alongshore momentum balance at the offshore point (figure 3-29a) develops more slowly. It still evolves from the initial forced inertial wave balance to a modulated Couette balance, then to pure Couette balance. However, the overall spin-up time is comparable to the flat bottom intermediate case. There is a slight difference between $z_{0} / h$ here and in the flat bottom intermediate case, but it does not affect the spin-up time much.

The cross-shore momentum balances at the inshore (figure 3-28b) and offshore (figure 3-29b) points have many similarities with the alongshore balances described above. The overall spin-up time at each point is predictable from the flat bottom case with the same local value of $u_{* s} / f h$, and the decay of oscillatory behavior is determined by the smallest $u_{* s} / f h$ values in the domain (i.e. $\mathrm{O}(10 \mathrm{hrs})$ offshore). At steady-state, the primary cross-shore balance is between the wind stress, pressure gradient and Coriolis force with bottom stress playing a secondary role, as it did in section 3.1. However, as in the flat bottom scenario, its importance in modifying the pressure gradient field can still have implications for the surface set-up.

\subsubsection{Bottom stress and drag tensor}

In the cross-shore direction, there is a finite downwind steady-state bottom stress at both points (figures $3-28 \mathrm{~b}$ and $3-29 \mathrm{~b}$ ). Not surprisingly, the cross-shore bottom stress is bigger at the onshore point than at the offshore point. This implies the magnitude of the bottom stress vector is slightly larger at the onshore point than at the offshore point because the alongshore bottom stress component is constant across the shelf. It also implies that the angle between the bottom stress and depth-averaged flow is slightly larger at the onshore point.

Since the steady-state bottom stress magnitude is larger and the depth-averaged speed is smaller at the onshore point, the resistance coefficient and estimated drag coefficient are larger at the onshore point. Figure 3-30 shows time series of the resistance coefficient at the two points. As expected, the steady-state values differ. The resistance coefficient onshore is the same as it was in the flat bottom shallow case since $u_{* s} / f h$ and $z_{0} / h$ both match. The resistance 
coefficient offshore is very different from the flat bottom intermediate case. Jenter and Madsen (1989) have shown that this difference is due to different roughness length to water depth and Ekman depth to water depth ratios at the two points.

There is also a cross-shelf difference in transient behavior of the resistance coefficient. The spin-up times and oscillations reflect those of the basic variables. The one exception seems to be an anomalously high value of $r$ at the offshore point during early times. To understand this, it is useful to examine time series of bottom stress magnitude (figure 3-31) and depth-averaged current speed (figure 3-32) at the offshore point from both the DRM and the $2 \frac{1}{2}$-D model. The two figures show that the large resistance coefficient corresponds to a small "hump" in the bottom stress concurrent with a relatively low velocity. It makes sense that, when the depth-averaged velocity is small, $r$ (and even more so $C_{d}$ ) is overly sensitive. Fortunately, the bottom stress is usually small at these times reducing the importance of the actual value of $r$.

Figure 3-33 shows the estimated drag coefficient at the two points as a function of time. The same basic behavior as the resistance coefficient is evident. The anomalous spike at the offshore point at early times also appears in this signal, but is even more pronounced because $C_{d}$ is roughly proportional to the inverse of depth-averaged velocity squared. The steady-state value of the drag coefficient at the onshore point is the same as the flat bottom shallow case, but the offshore value is smaller than the flat bottom intermediate case. The difference is primarily due to the difference in $z_{0} / h$ (Jenter and Madsen; 1989). This is supported by the fact that there was no variation in $C_{d}$ among the flat bottom cases which all had the same roughness length to water depth ratio (figure 3-18).

The angular behavior of the drag tensor, bottom stress and depth-averaged velocity is depicted in figures 3-34 and 3-35. At both points, the $\beta$ starts out at $45^{\circ}$ and decreases linearly for the short period that there is no bottom friction or pressure gradient. After these terms develop, it drops more rapidly until the flow is approximately alongshore. It then oscillates with the cross-shore velocity until reaching exactly $0^{\circ}$ at steady-state.

A rapid approach to constant $\theta$ can be seen in both figures. Long before $\alpha$ and $\beta$ reach steady-state, their difference does. This is especially true in figure 3-34 which has important implications for using the $2 \frac{1}{2}$-D model to model time-dependent forcing problems. The rapid angular response of the drag tensor means that it will not place undue restrictions on the $2 \frac{1}{2}$-D 


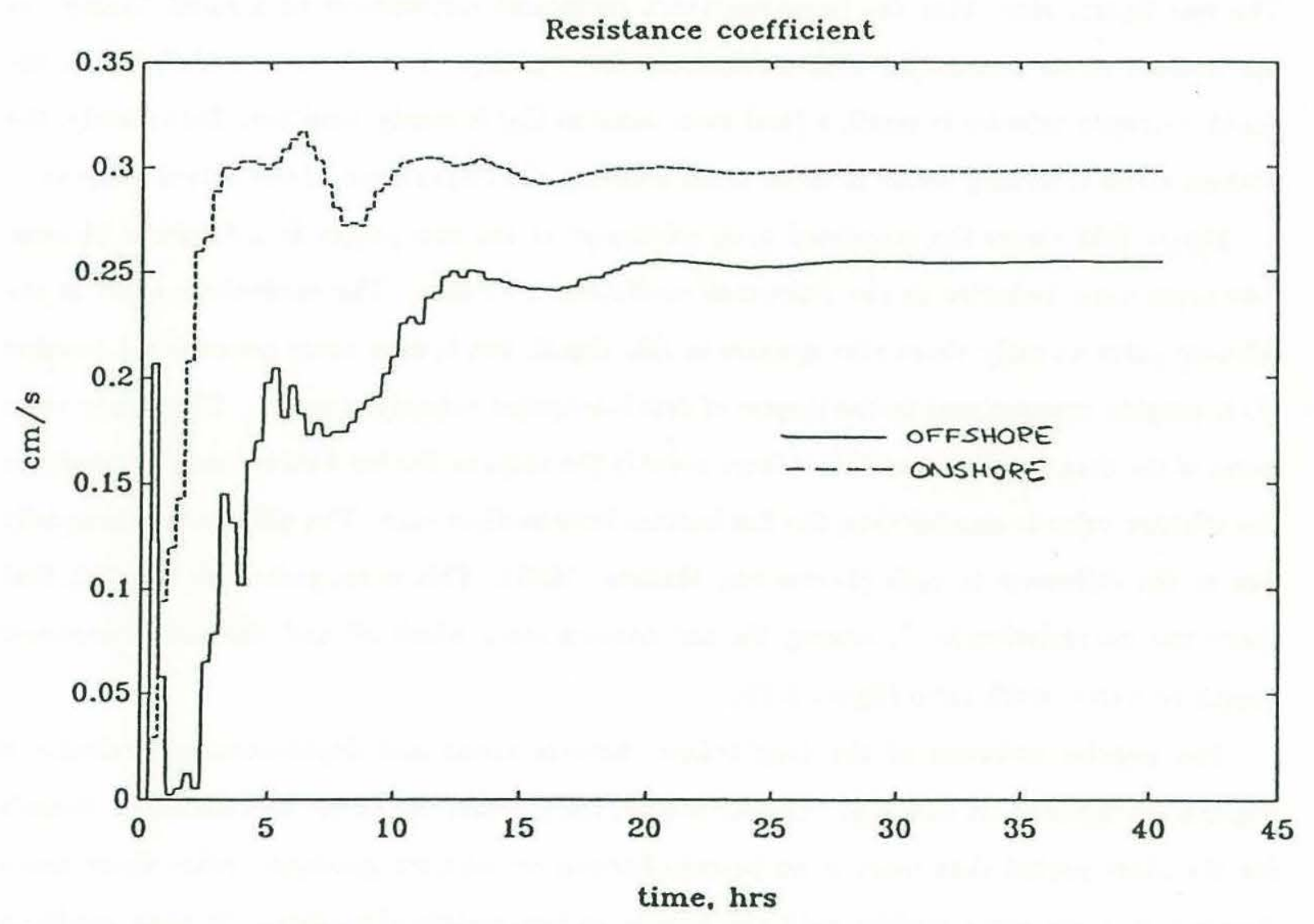

Figure 3-30: Time series of resistance coefficient at the onshore and offshore points. 


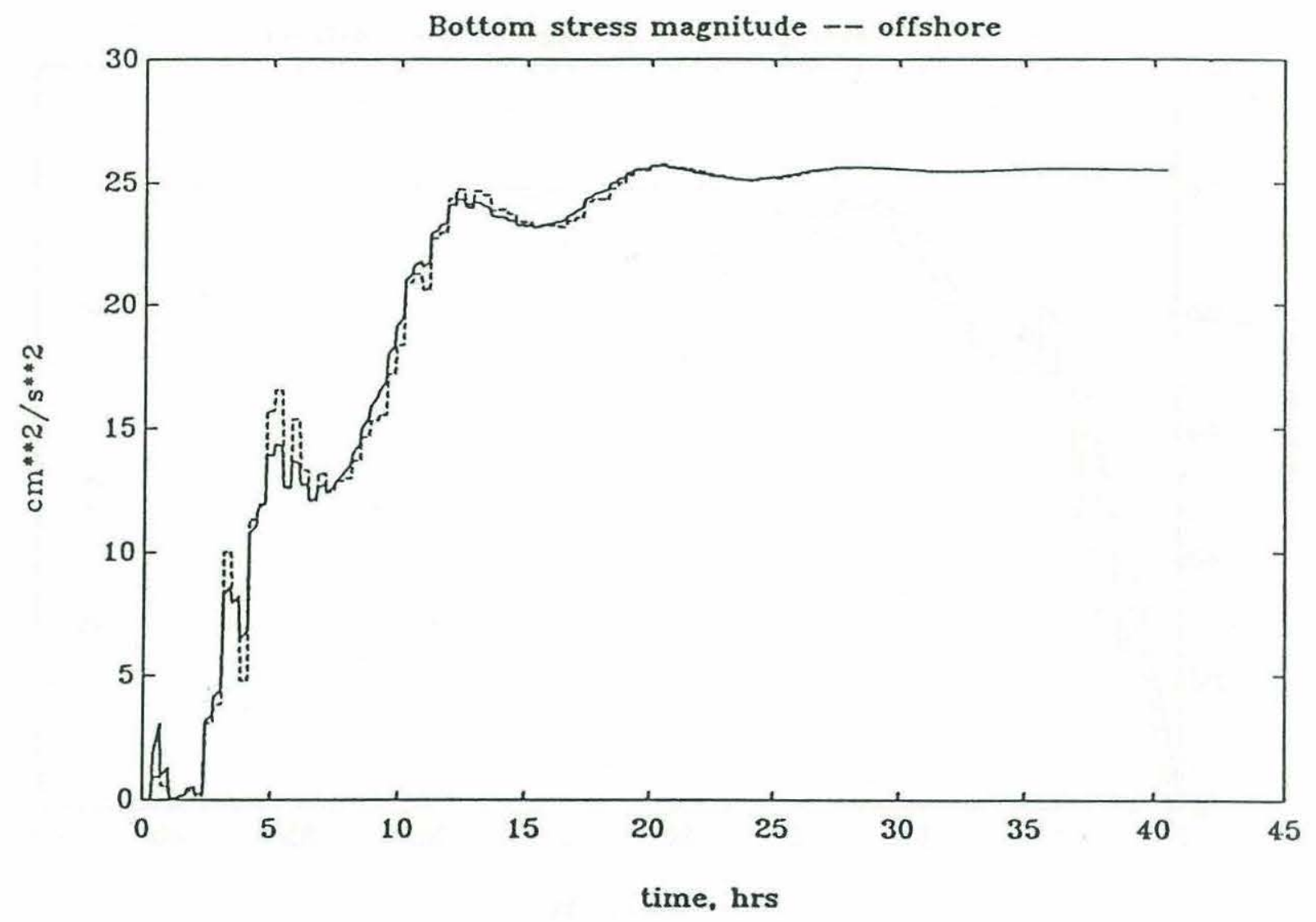

Figure 3-31: Time series of kinematic bottom stress magnitude, $\left|\vec{\tau}_{b}\right| / \rho$, at the offshore point. Solid line indicates DAM values and dashed line indicates DRM values. 


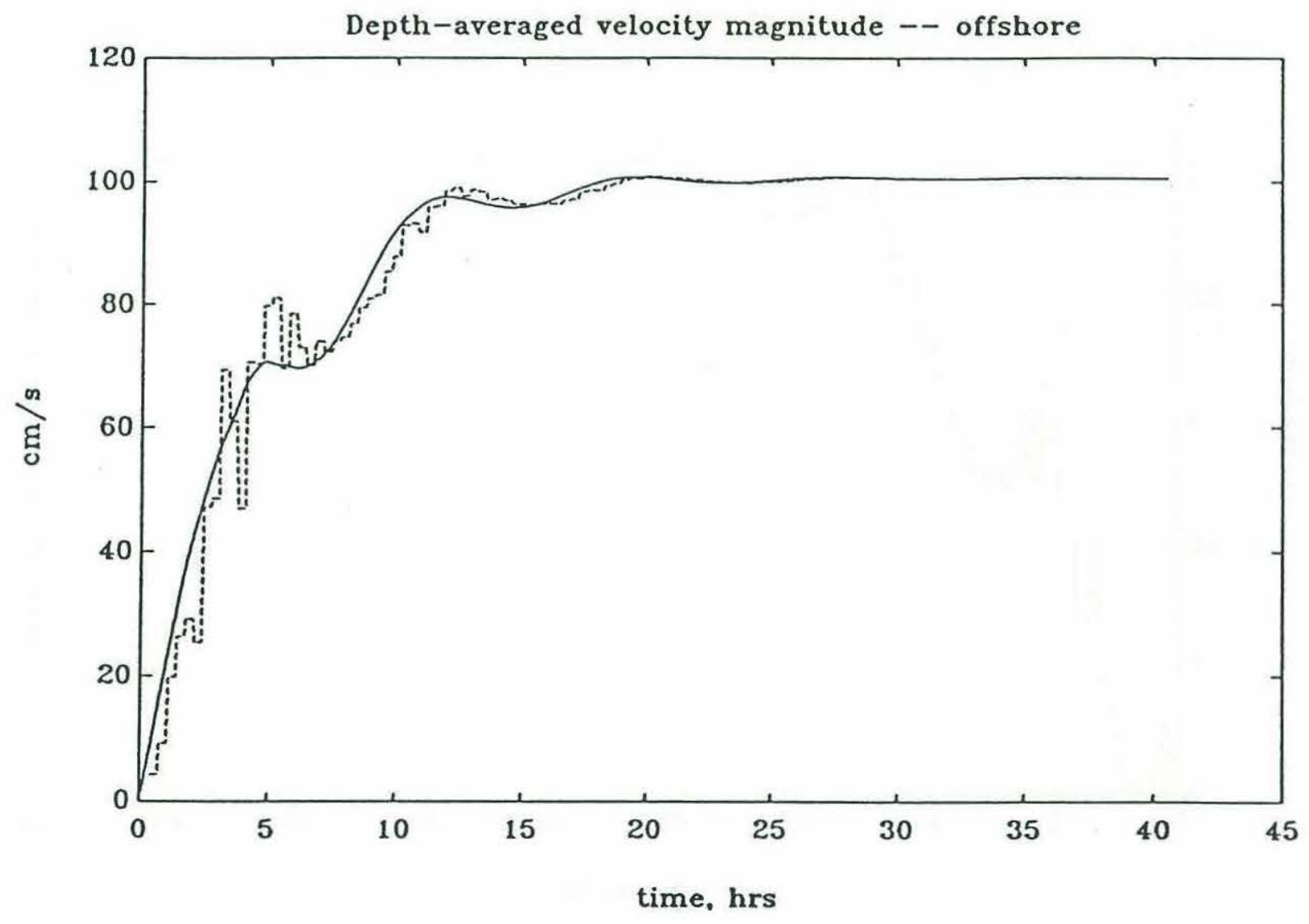

Figure 3-32: Time series of depth-averaged velocity magnitude, $|\vec{U}| / h$, at the offshore point. Solid line indicates DAM values and dashed line indicates DRM values. 


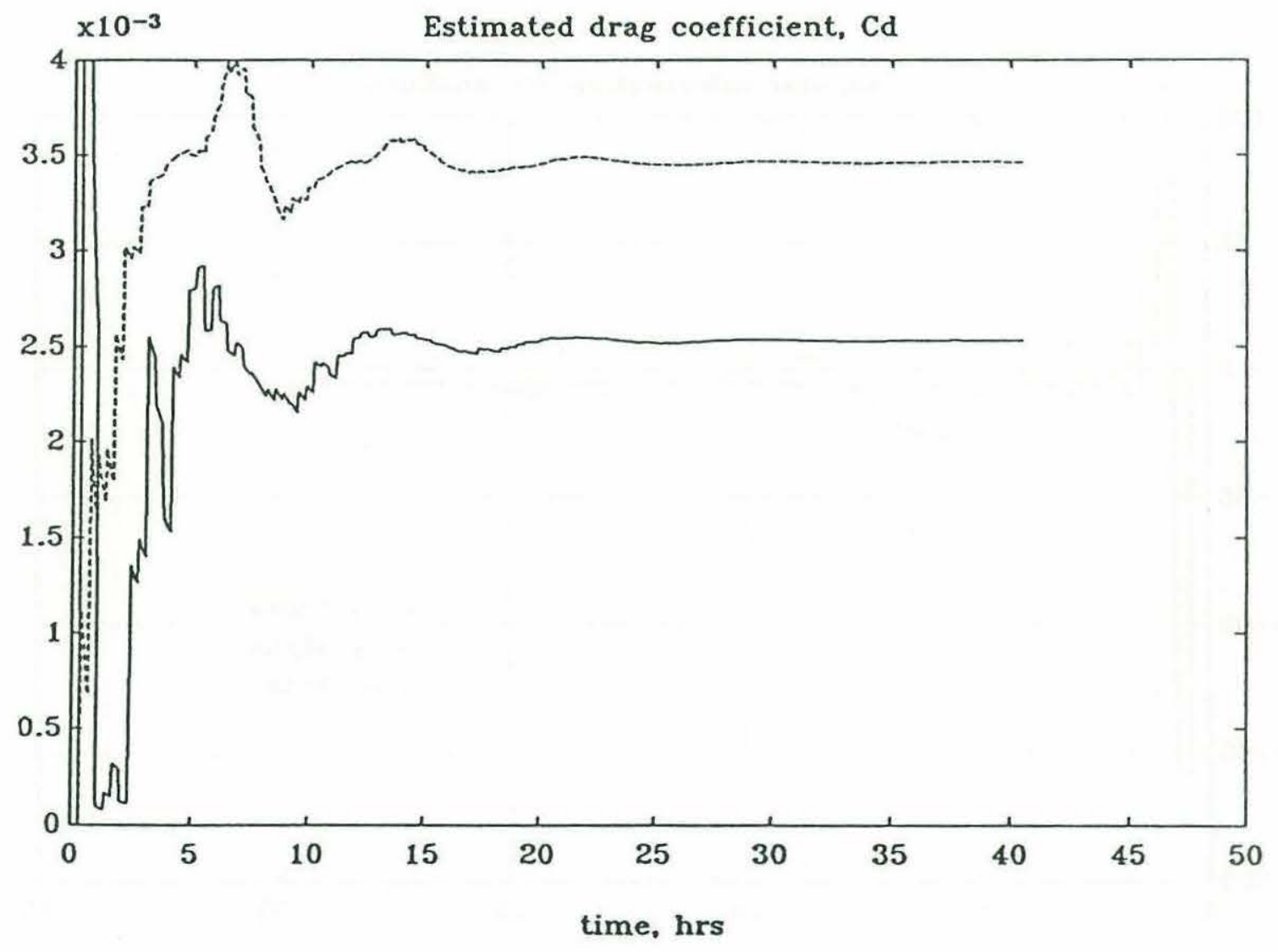

Figure 3-33: Time series of estimated drag coefficient at the offshore (solid line) and onshore (dashed line) points. The spike in the offshore point signal is truncated at .004 although its actual maximum is $C_{d} \approx .023$. 


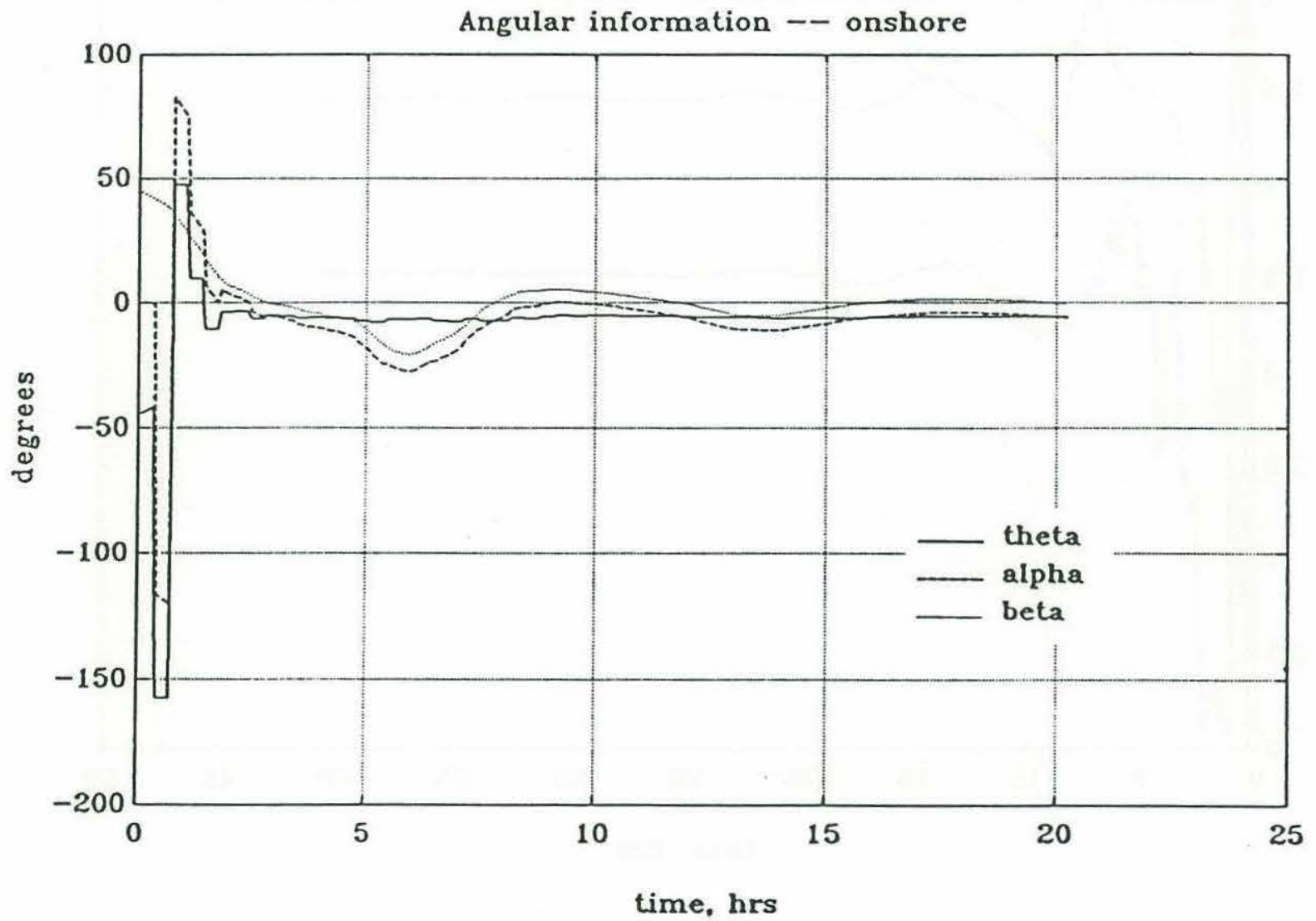

Figure 3-34: Time series of anguiar intormation at the onshore point. 


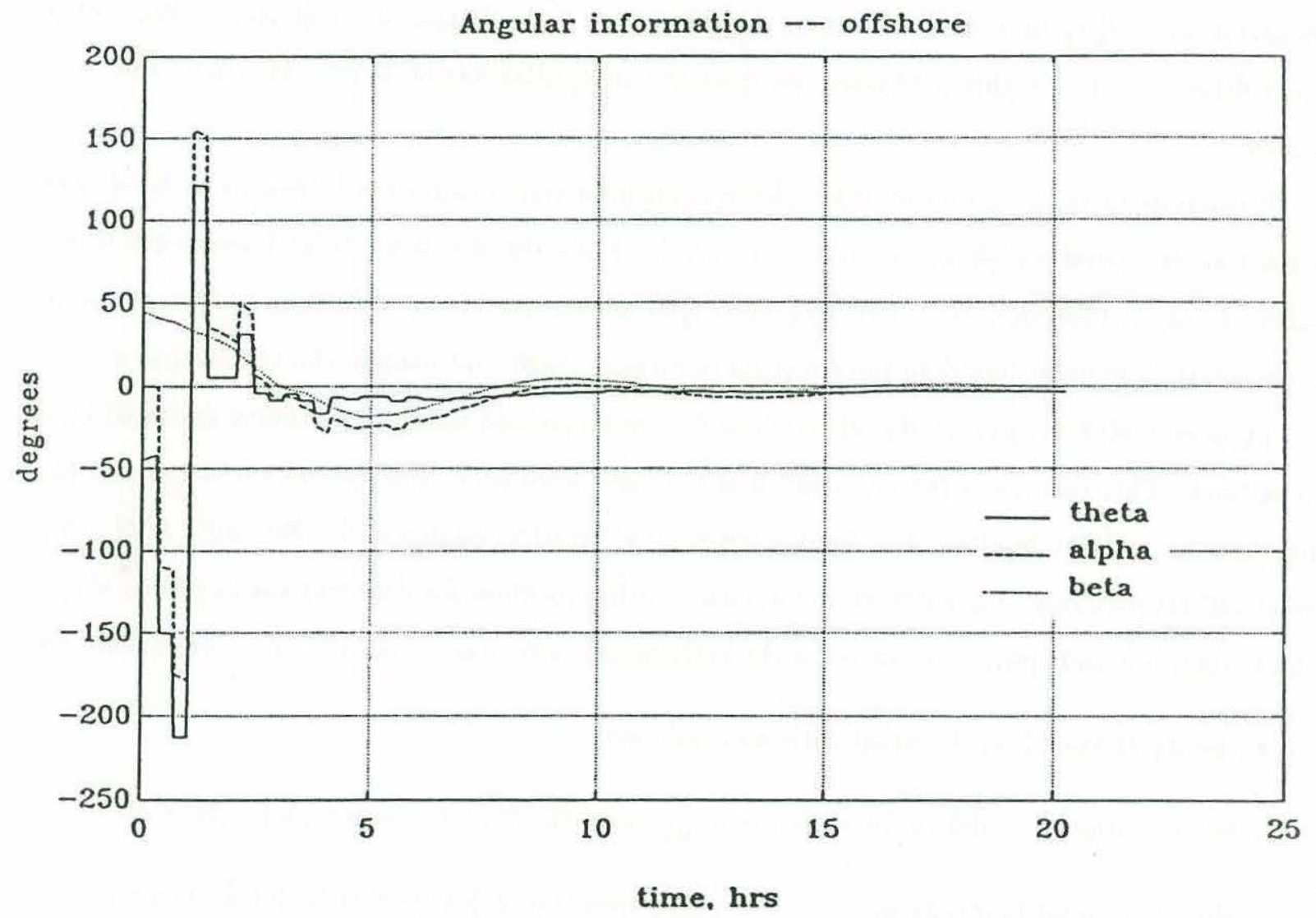

Figure 3-35: Time series of angular information at the offshore point. 
model's ability to respond to slow temporal variations in forcing.

With variable depth, there is now a difference among steady-state $\theta$ values across the shelf. Onshore the value is roughly $-6^{\circ}$ as it was in the flat bottom shallow case. Offshore it is closer to $-3^{\circ}$ which is approximately the flat bottom intermediate value. The difference in $\theta$ across the shelf was pointed out earlier in the momentum balance discussions.

\subsubsection{Differences between traditional and $2 \frac{1}{2}-\mathrm{D}$ model results}

Results from the flat bottom cases showed that failure to include time-dependence and directional variability in a drag law leads to differences in both spin-up and steady-state of a wind-driven shelf. In this scenario, the question of spatial variability in the drag law was added.

Numerous tests (not reported here) have confirmed that similar conclusions can be drawn regarding temporal variability of the drag law for both the flat bottom and sloping bottom cases. Namely, that spin-up is faster in the $2 \frac{1}{2}$-D model with a variable drag tensor provided its magnitude remains less than the constant resistance coefficient used in the traditional model.

Figures 3-36-3-41 specifically address the failure to include spatial variability in traditional drag laws. This was not a factor in the first scenario because of the spatial uniformity of the topography and the forcing. The figures correspond to wind angles of $0^{\circ}, 30^{\circ}, 60^{\circ}, 120^{\circ}, 150^{\circ}$ and $180^{\circ}$ respectively. They depict steady-state surface profiles for different assumptions about the magnitude and spatial variation of the resistance coefficient. The four lines correspond to:

- the $2 \frac{1}{2}$-D model result (solid with no symbols)

- the traditional model result with $r=r_{\min }$ from the $2 \frac{1}{2}$-D model (solid with x's)

- the traditional model result with $r=r_{\max }$ from the $2 \frac{1}{2}$-D model (solid with o's)

- a modified traditional model with $r=r(y)$ from the $2 \frac{1}{2}$-D model (solid with *'s)

Note, the last three assume there is no angle between the depth-averaged velocity and the bottom stress (i.e. $\theta=0$ ).

It is clear from the figures that qualitative behavior of the steady-states is not altered by the choice of bottom stress formulation. However, quantitative behavior is sometimes strongly 


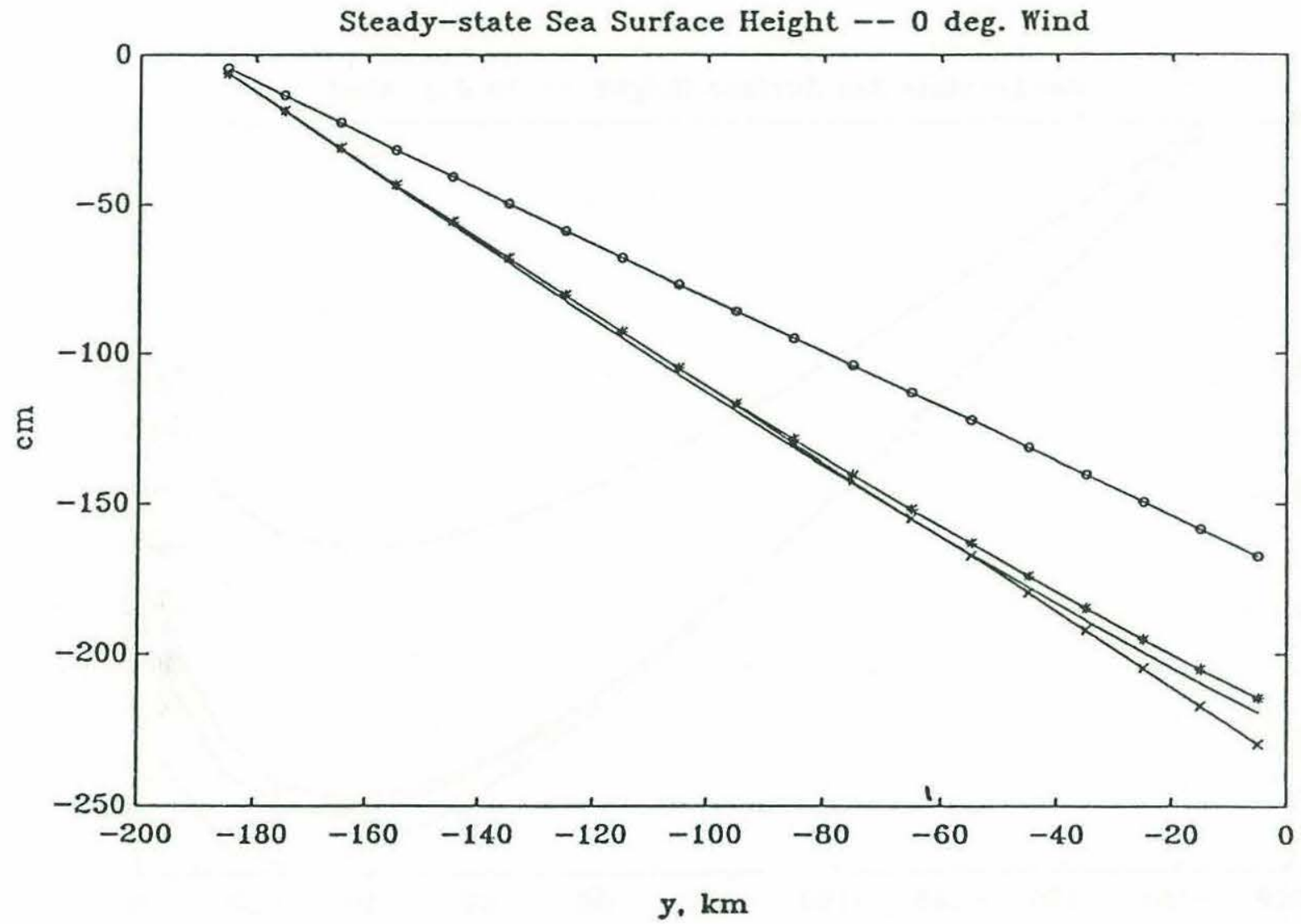

Figure 3-36: Steady-state surface profile for a wind angle of $0^{\circ}$. Curves are for the $2 \frac{1}{2}$-D model $(-)$, traditional model with $r=r_{\min }(\mathrm{x}-\mathrm{x})$, traditional model with $r=r_{\max }(\mathrm{o}-\mathrm{o})$ and traditional model with $r=r(y)\left({ }^{*}-^{*}\right)$. 


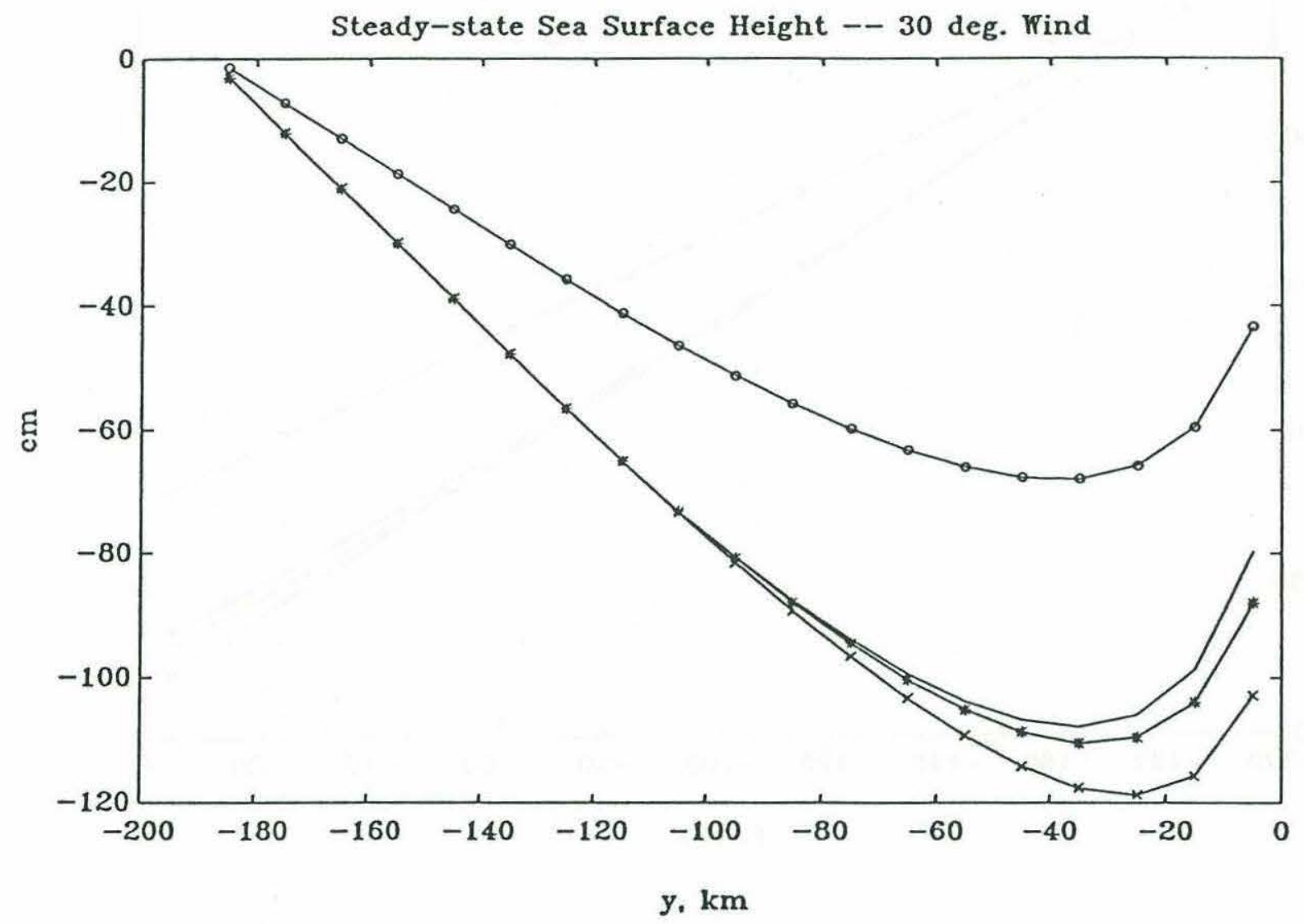

Figure 3-37: Steady-state surface profile for a wind angle of $30^{\circ}$. Curves are for the $2 \frac{1}{2}$-D model $(-)$, traditional model with $r=r_{\min }(\mathrm{x}-\mathrm{x})$, traditional model with $r=r_{\max }(\mathrm{o}-\mathrm{o})$ and traditional model with $r=r(y)\left({ }^{*}-{ }^{*}\right)$. 


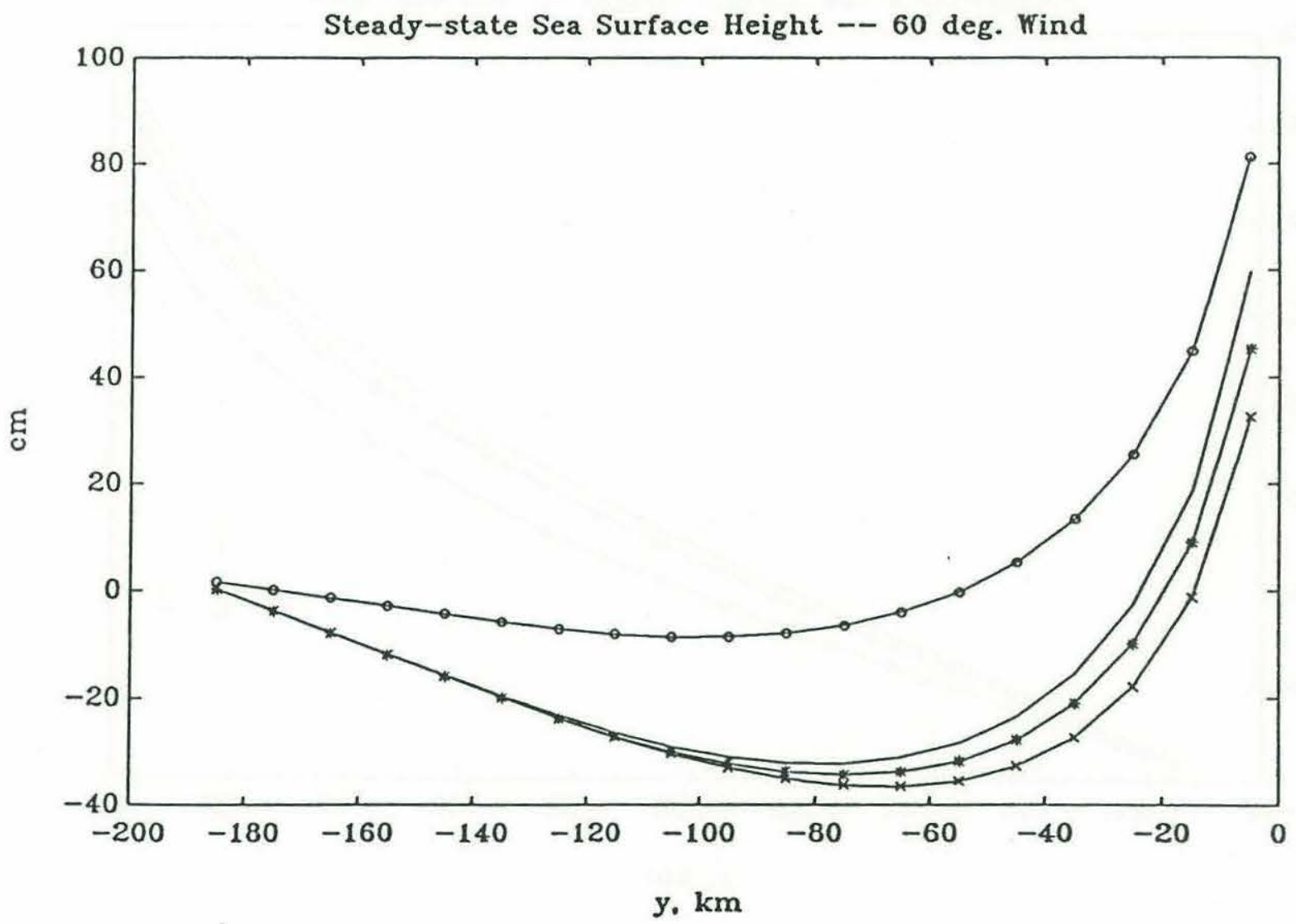

Figure 3-38: Steady-state surface profile for a wind angle of $60^{\circ}$. Curves are for the $2 \frac{1}{2}$-D model $(-)$, traditional model with $r=r_{\min }(\mathrm{x}-\mathrm{x})$, traditional model with $r=r_{\max }(\mathrm{o}-\mathrm{o})$ and traditional model with $r=r(y)\left({ }^{*}-^{*}\right)$. 


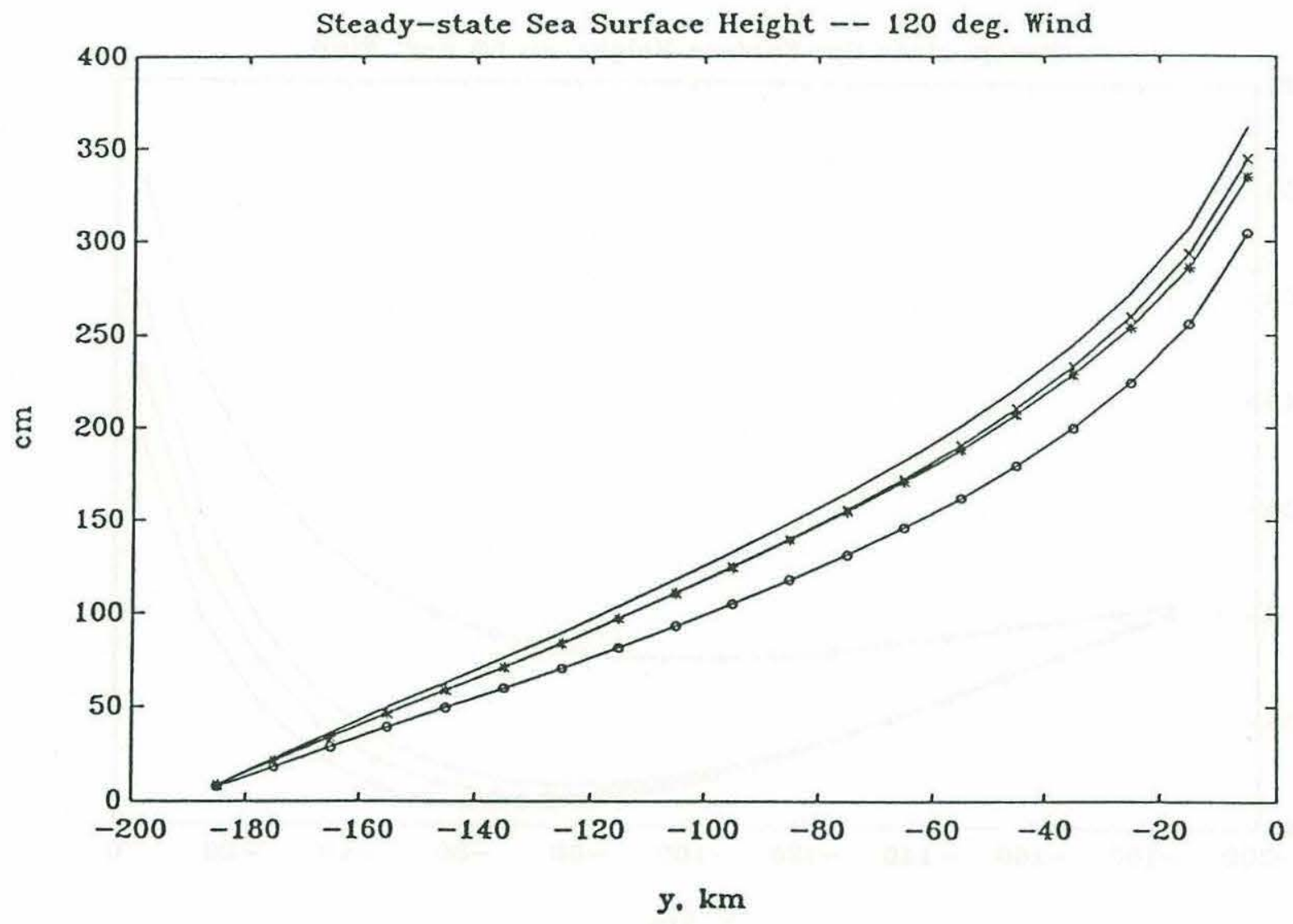

Figure 3-39: Steady-state surface profile for a wind angle of $120^{\circ}$. Curves are for the $2 \frac{1}{2}$-D model (-), traditional model with $r=r_{\min }(\mathrm{x}-\mathrm{x})$, traditional model with $r=r_{\max }(\mathrm{o}-\mathrm{o})$ and traditional model with $r=r(y)\left(*^{*}{ }^{*}\right)$. 


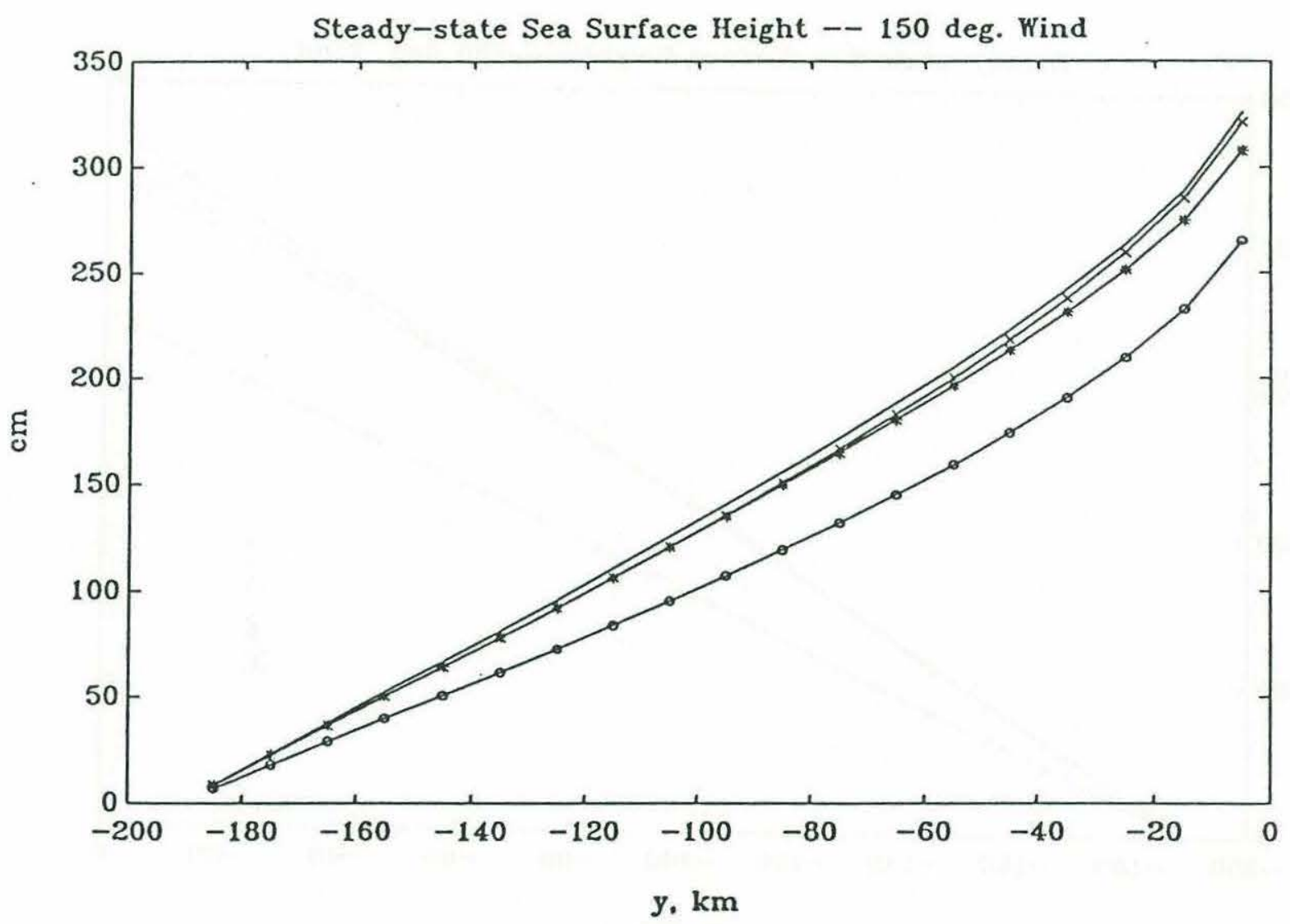

Figure 3-40: Steady-state surface profile for a wind angle of $150^{\circ}$. Curves are for the $2 \frac{1}{2}-\mathrm{D}$ model $(-)$, traditional model with $r=r_{\min }(\mathrm{x}-\mathrm{x})$, traditional model with $r=r_{\max }(\mathrm{o}-\mathrm{o})$ and traditional model with $r=r(y)\left({ }^{*}-^{*}\right)$. 


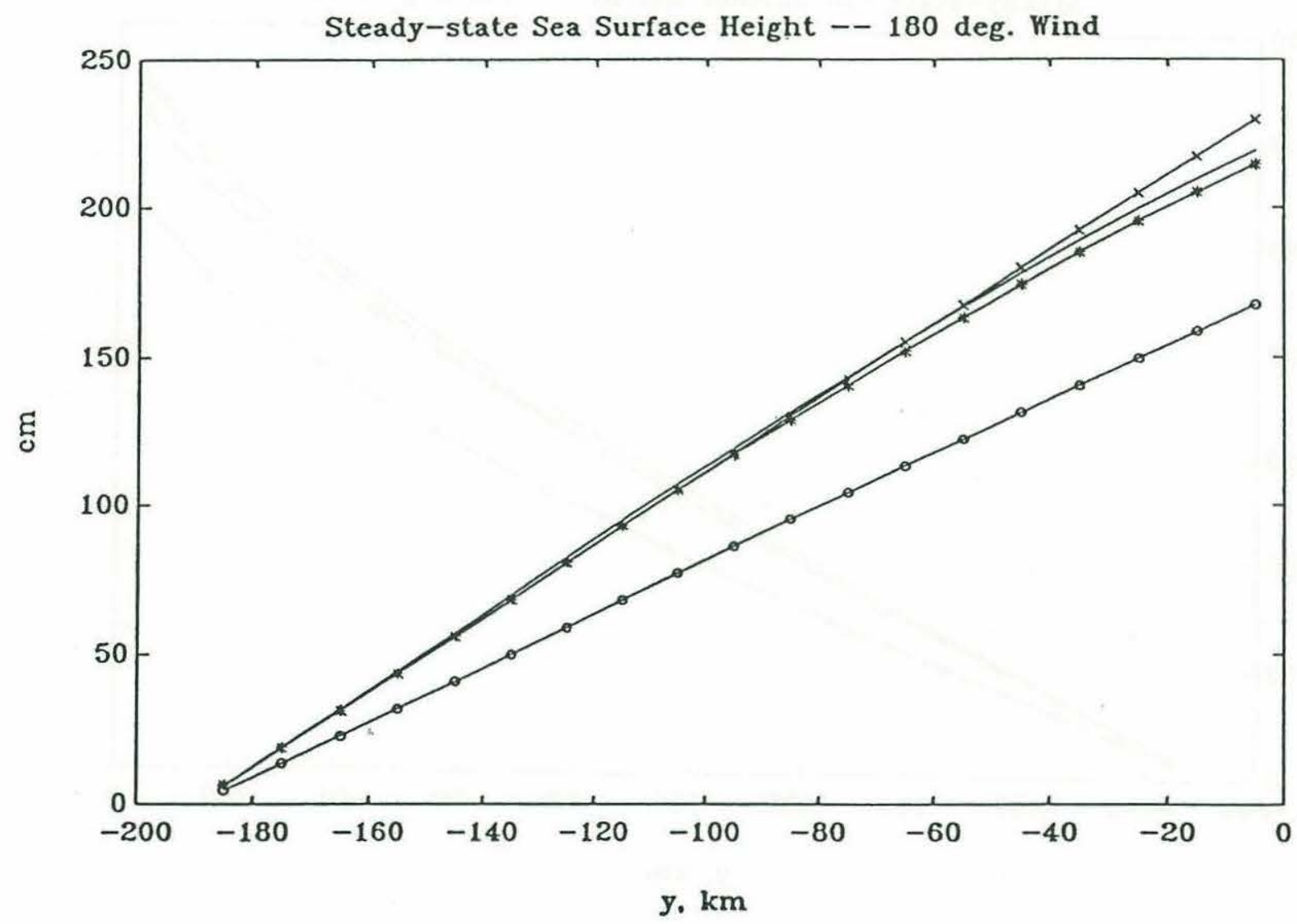

Figure 3-41: Steady-state surface profile for a wind angle of $180^{\circ}$. Curves are for the $2 \frac{1}{2}-\mathrm{D}$ model (-), traditional model with $r=r_{\min }(\mathrm{x}-\mathrm{x})$, traditional model with $r=r_{\max }(0-0)$ and traditional model with $r=r(y)\left({ }^{*}{ }^{*}\right)$. 
affected. The difference between curves in each figure gives an indication of error magnitudes that might be expected from ignoring $\theta$ and using a spatially constant resistance coefficient (assuming it was chosen to fall in the range of values determined by the $2 \frac{1}{2}$-D model physics!).

For this particular model domain, choosing a constant resistance coefficient equal to the minimum $r$ value from the $2 \frac{1}{2}$-D model is better than choosing $r=r_{\max }$. Better agreement for the small $r$ value is understandable since $r$ predicted by the $2 \frac{1}{2}$-D model does not vary linearly across the shelf. The onshore region in which $r \approx r_{\max }$ is smaller than the offshore region in which $r \approx r_{\min }$.

Since all solutions are pinned at the offshore boundary (i.e. $\zeta=0$ at $y=-L$ ), the biggest differences in surface height are away from the shelf edge. Note that agreement of surface height at any point is an integral effect. It implies correct reproduction of the average crossshore pressure gradient seaward of that point. This explains why the profiles corresponding to $r=r_{\min }$ correspond "better" with the $2 \frac{1}{2}$-D model solutions. The curves which use $r=r_{\max }$ deviate from the $2 \frac{1}{2}$-D results immediately inshore of the shelf edge. Therefore, the integrated effect is seen all the way across the shelf. The fact that the large resistance coefficient is more appropriate onshore is illustrated by the $r=r_{\max }$ curves being more parallel to the $2 \frac{1}{2}$-D model results near the coast and having maximum differences from the $2 \frac{1}{2}$ - $\mathrm{D}$ model results in the middle of the shelf.

It is interesting to note that the $r=r_{\min }$ and $r=r_{\max }$ curves only bracket the $2 \frac{1}{2}$-D model curve for wind angles less than $90^{\circ}$. Also, the $r_{\max }$ line lies above the $r=r_{\min }$ line in this range. For angles between $90^{\circ}$ and $180^{\circ}$, both constant $r$ curves lie below the $2 \frac{1}{2}$-D model curve and the $r=r_{\max }$ line lies below the $r=r_{\min }$ line.

The reason that the profiles do not bracket the $2 \frac{1}{2}$-D model profile is that $\theta=0$ in all of the constant $r$ cases, leaving an additional effect unaccounted for. This is apparent from examining the fourth curve in each plot. This curve was generated from a modified traditional model where the resistance coefficient was allowed to vary in $y$ in exactly the same way as the steady-state drag tensor magnitude from the $2 \frac{1}{2}$-D model. For angles less than $90^{\circ}$, allowing $r$ to vary spatially improves the agreement between the $2 \frac{1}{2}$ - $\mathrm{D}$ model and the traditional model. There is still some disagreement caused by assuming $\theta=0$. In fact, the variable $r$ curves for angles greater than $90^{\circ}$ show worse agreement with the $2 \frac{1}{2}$ - $\mathrm{D}$ model results than do the 
$r=r_{\min }$ curves. Apparently, the two sources of error, using constant $r$ across the shelf and setting $\theta=0$, cancel each other to some extent in this range.

Inversion of the $r=r_{\max }$ and $r=r_{\min }$ lines is caused by Coriolis accelerations being directed offshore for wind angles less than $90^{\circ}$ and onshore for angles greater than $90^{\circ}$. Since larger $r$ yields smaller alongshore velocities, the cross-shore Coriolis force is smaller for $r=$ $r_{\max }$. Therefore, when one wind direction is compared to its compliment, the pressure gradient does not change as much for $r=r_{\max }$ as for $r=r_{\min }$, and the $r=r_{\min }$ curve jumps over the $r=r_{\max }$ curve.

\subsection{Linearly-Varying Depth, Sinusoidal Coastline}

This section describes wind-driven flow over a shelf whose depth varies linearly away from a sinusoidal coastline. The sinusoidal variation is chosen in order to induce alongshore $(x)$ as well as cross-shore $(y)$ variations in drag tensor.

\subsubsection{Model domain and wind stress field}

The model domain for this case is essentially rectangular (figure 3-1) with a larger alongshore dimension than was used in sections 3.1 and 3.2. The domain boundary is straight on three sides, but sinusoidal along the coast. The domain extends $62 \Delta x$ (one wavelength of the coastline $+\Delta x)$ in the $x$ direction and 19.5 $\Delta x$ across-shore. The amplitude of the coastline variation is $5 \Delta x$ where again $\Delta x=10 \mathrm{~km}$.

The bathymetry varies as it did in section 3.2 . At each point along the coast, the depth increases from $5 \mathrm{~m}$ to $70 \mathrm{~m}$ by $5 \mathrm{~m}$ per grid point in the $y$ direction. The amount of flat area offshore $(h=70 \mathrm{~m})$ is determined by the proximity of the end of the linearly-varying region to the straight shelf edge. The shelf edge is positioned so that the narrowest part of the model domain has no flat region. The bottom is covered by roughness elements corresponding to $z_{0}=1 \mathrm{~cm}$, meaning $z_{0} / h$ varies from $2 \times 10^{-3}$ near the coast to $1.4 \times 10^{-4}$ offshore with sinusoidal contours of constant $z_{0} / h$. The wind stress is the same as was used in the previous section (i.e. corresponding to a $33 \mathrm{~m} / \mathrm{s}$ speed and $45^{\circ}$ direction). It is important to note that all angles in this section are measured relative to the $x$-axis, not a line parallel to the local 
coastline. Due to the identical cross-shore bathymetry and wind speed, the model time step $(243 \mathrm{~s})$, diffusion time scales $(315-4375 \mathrm{~s})$, and update frequency (once every $5 \Delta t$ ) are the same as in the linearly-varying depth straight coastline case.

One of the most important reasons for examining this scenario is that the angle between the wind and the coast is not constant, but varies with alongshore position. Given the prescribed coastline configuration, the angle that the wind makes with the coast varies from about $15^{\circ}$ on the leeward side of the coastline's "crest" to $75^{\circ}$ on the windward side.

\subsubsection{Surface height and depth-averaged velocity}

Figures 3-42 and 3-43 show contour plots of sea surface height at various times during spinup and at steady-state. The time difference between the initial plots is approximately 2.4 hrs $(35 \Delta t)$. Therefore, they adequately depict the overall approach to steady-state while somewhat under-resolving the seiching. As with the previous cases, the water level within the entire domain rises initially during the propagation of the surface signal away from the coast. However, it does not rise uniformly along the coast since the angle between the wind and the coast varies. The largest rise is on the windward shore of the "crest" where the wind angle relative to the shore is the largest.

After the initial rise, the sea surface height drops sharply as an upwind pressure gradient develops. The surface height remains positive only within a small area near the windward shore, while dropping far below its original level in the rest of the domain. Once this basic configuration is reached, it persists to steady-state. There is still some low amplitude temporal oscillation and movement of the bulge along the coast. This is evidenced by small differences in contour locations among the later "snapshots".

The steady-state sea surface contains a thin steep band of positive values along the windward side of the shoreline "crest". Here, the contours are nearly perpendicular to the wind indicating that the pressure gradient is almost straight upwind. The leeward side of the "crest" shows a large depression in the sea surface extending well offshore. In shallow water, the pressure gradient is again in the direction of the wind. This is particularly true on the left side of the depression where the sea surface slope opposes the wind. On the other side of the depression, the pressure gradient is downwind and the contour lines are not nearly as well aligned. The 


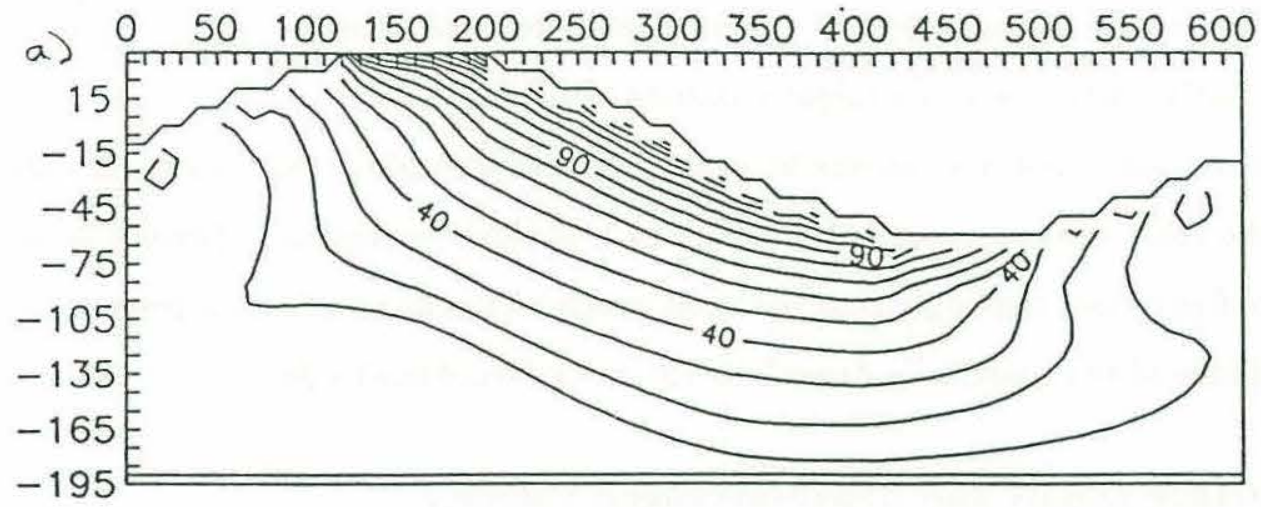

b) $450 \quad 50 \quad 100 \quad 150200250300 \quad 350 \quad 400450500550600$

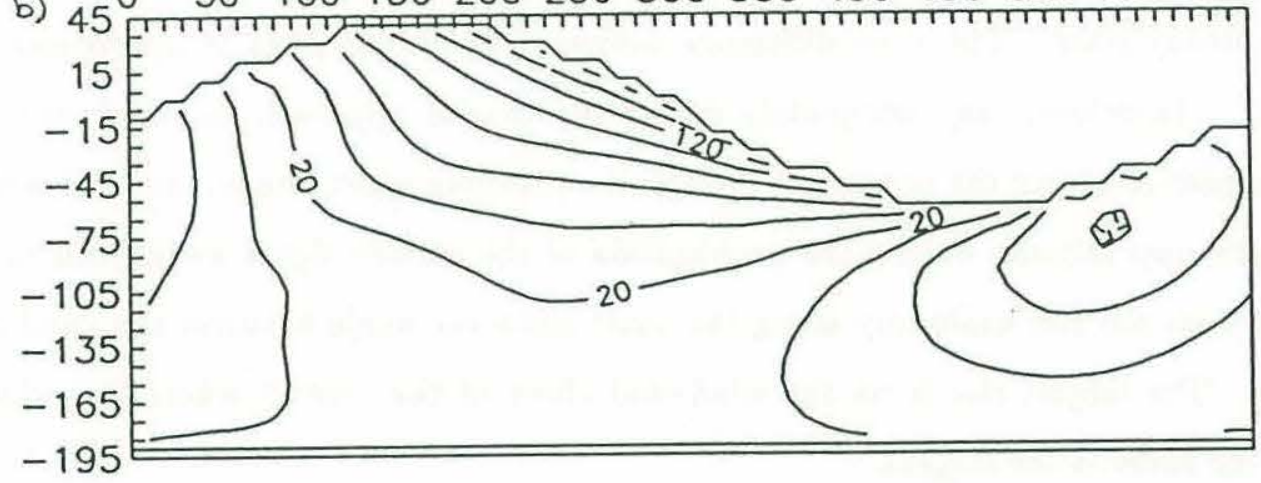

c) $450 \quad 50 \quad 100 \quad 150200250300350 \quad 400 \quad 450500550600$

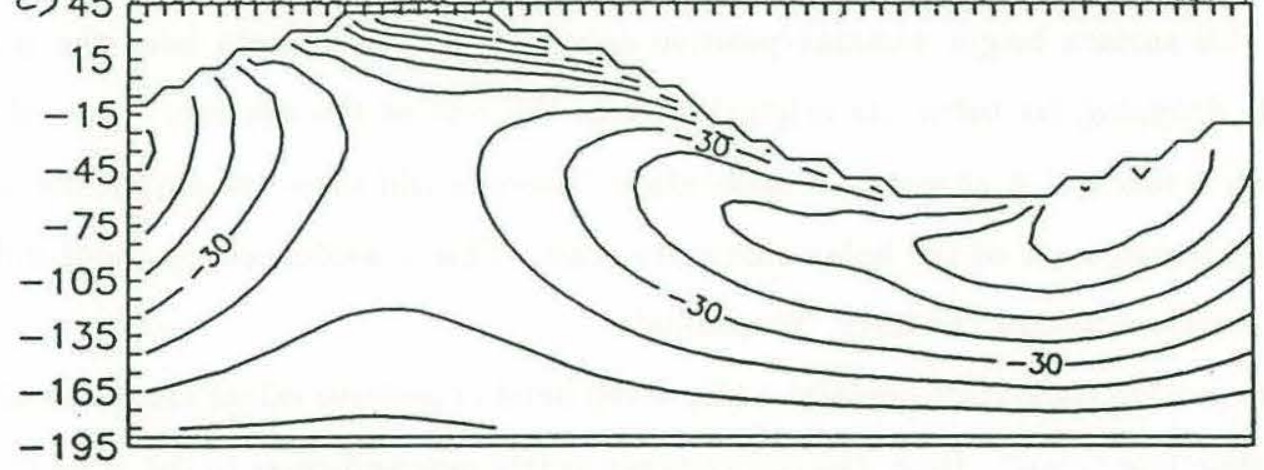

Figure 3-42: Contours of surface elevation at a) 2.4 , b) 4.8 and c) $7.2 \mathrm{hrs}$. 

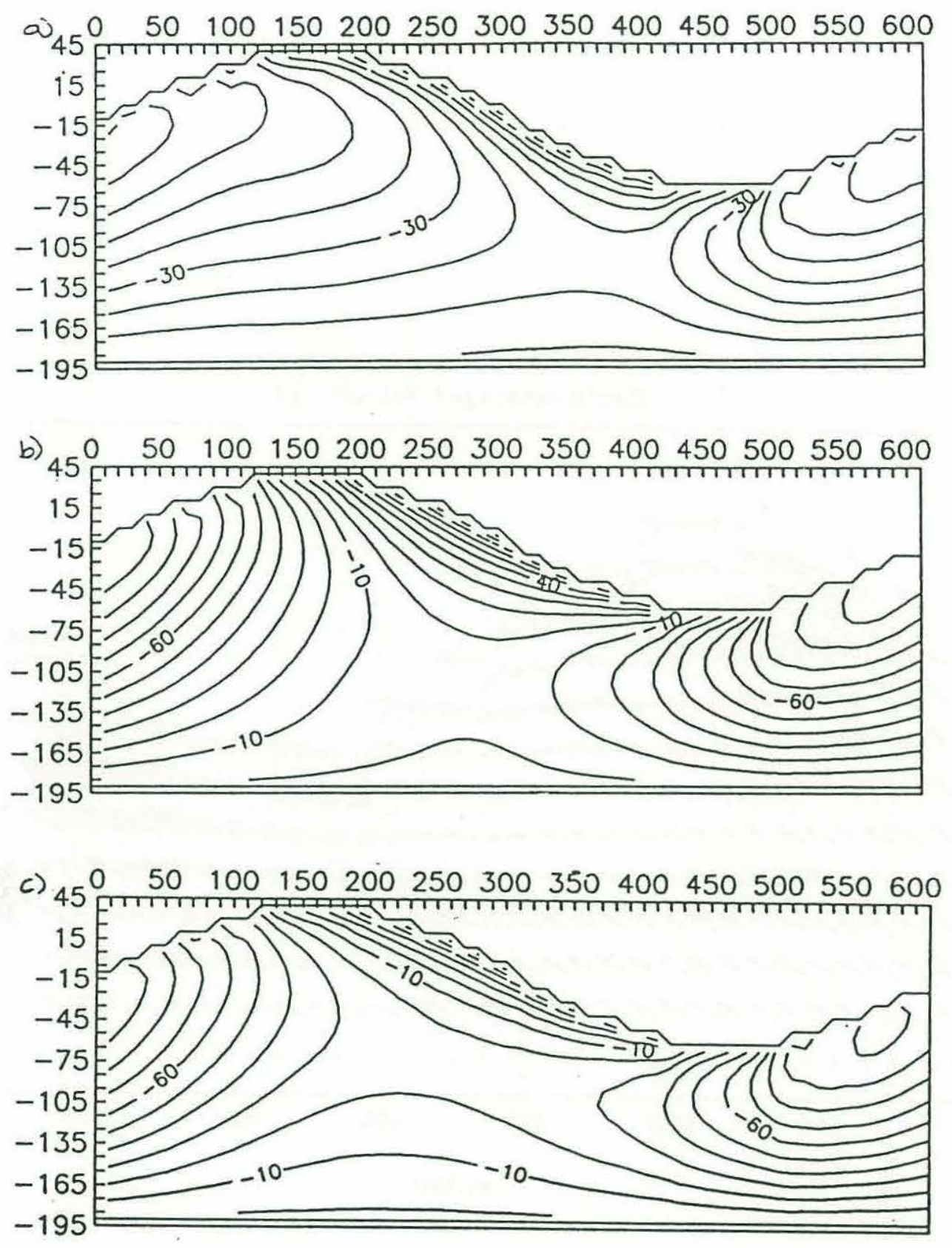

Figure 3-43: Contours plots of surface elevation at a) 9.6 and b) $12 \mathrm{hrs}$ and c) steady-state. 


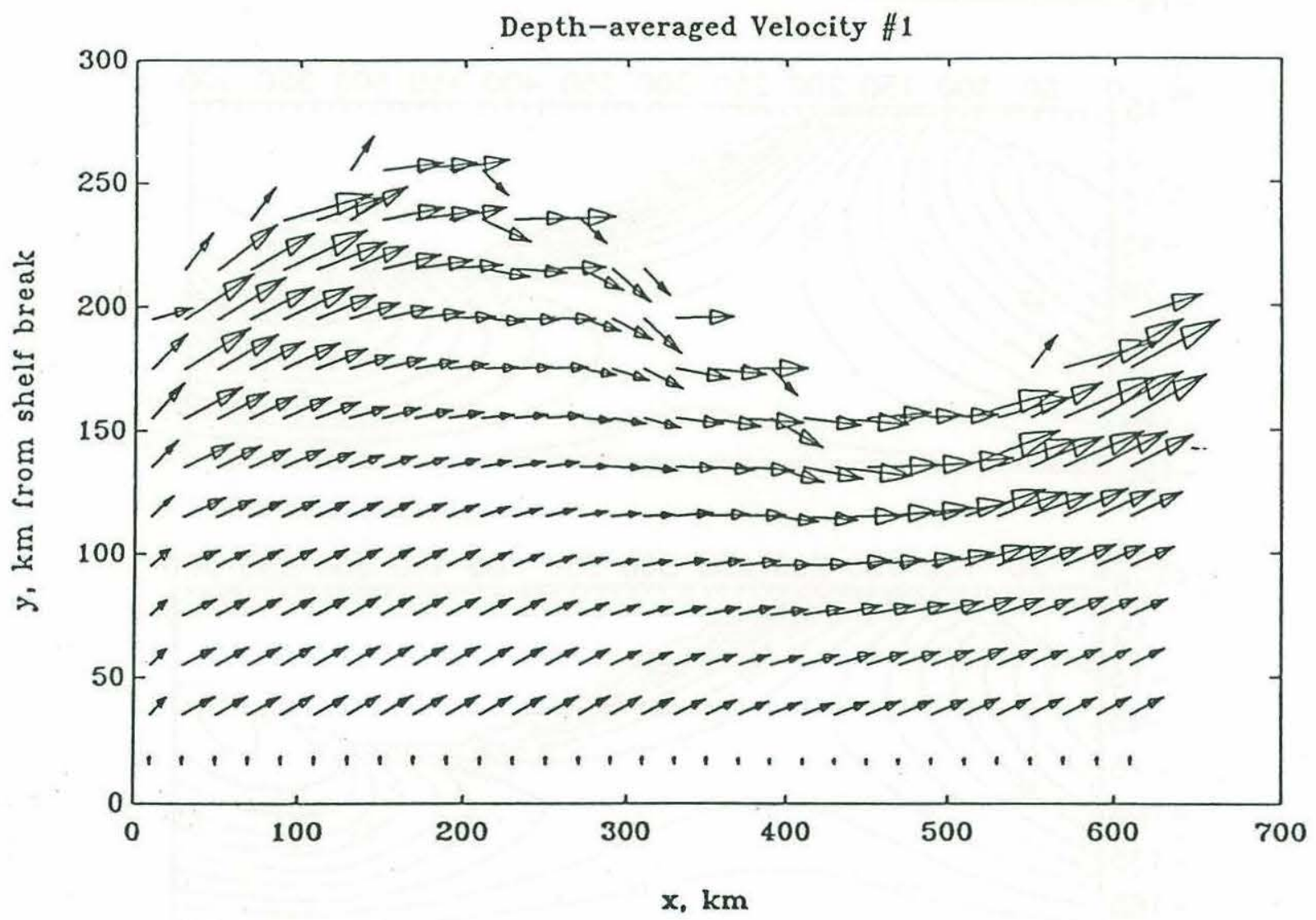

Figure 3-44: Depth-averaged velocity field at $2.4 \mathrm{hrs}$. Scale: $1 \mathrm{~cm}=60 \mathrm{~cm} / \mathrm{s}$. 


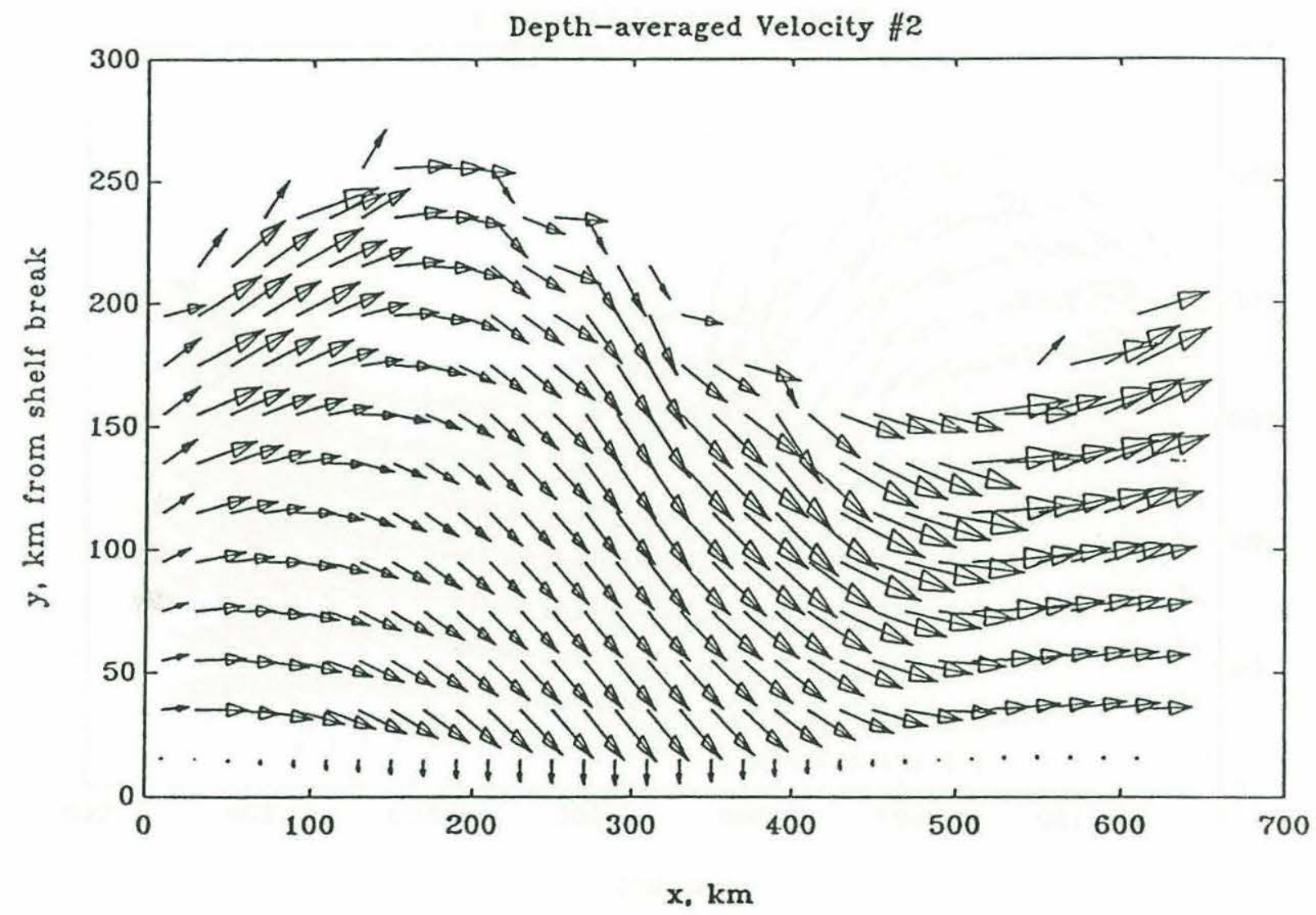

Figure 3-45: Depth-averaged velocity field at $4.8 \mathrm{hrs}$. Scale: $1 \mathrm{~cm}=60 \mathrm{~cm} / \mathrm{s}$. 


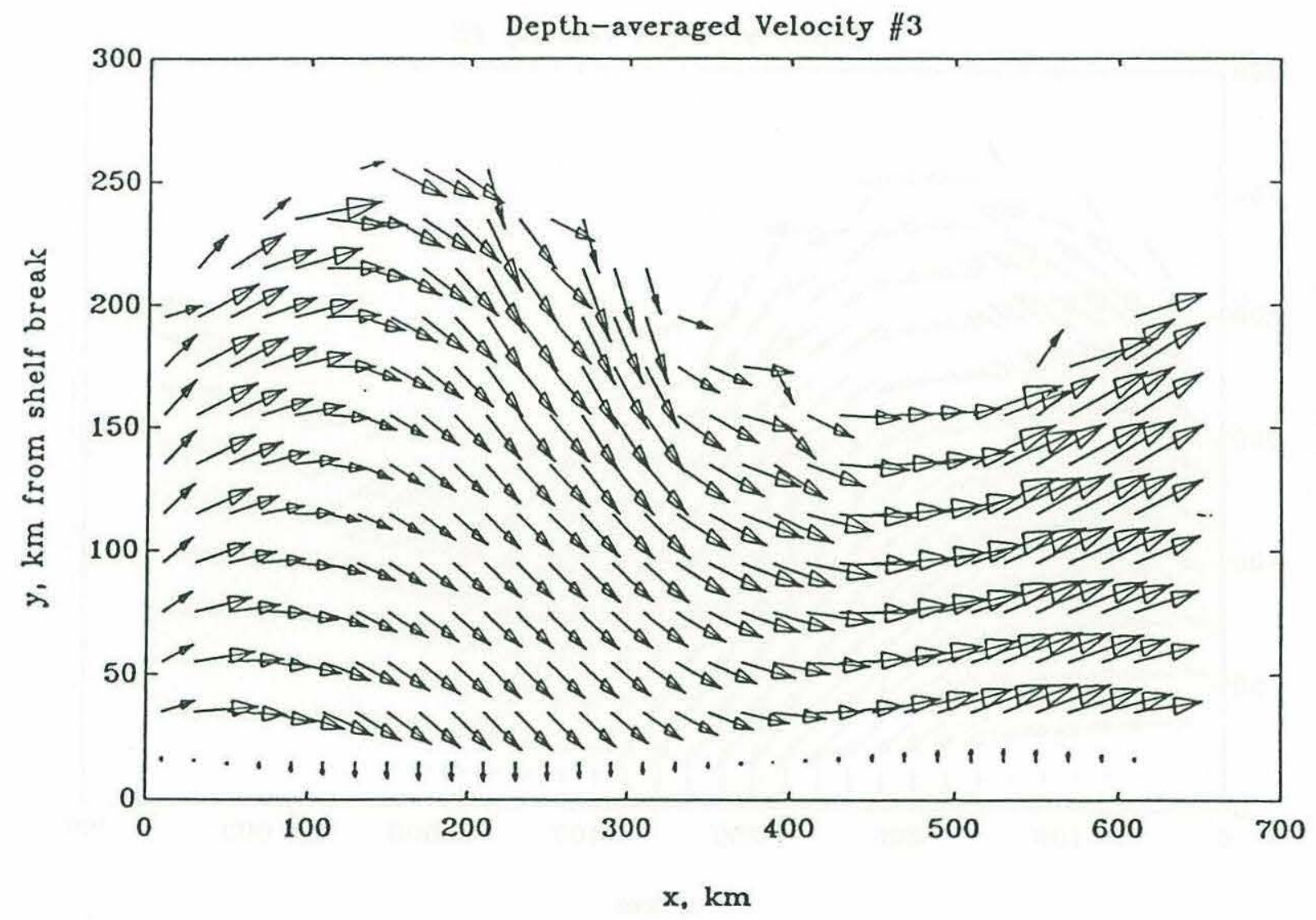

Figure 3-46: Depth-averaged velocity field at $7.2 \mathrm{hrs}$. Scale: $1 \mathrm{~cm}=60 \mathrm{~cm} / \mathrm{s}$. 


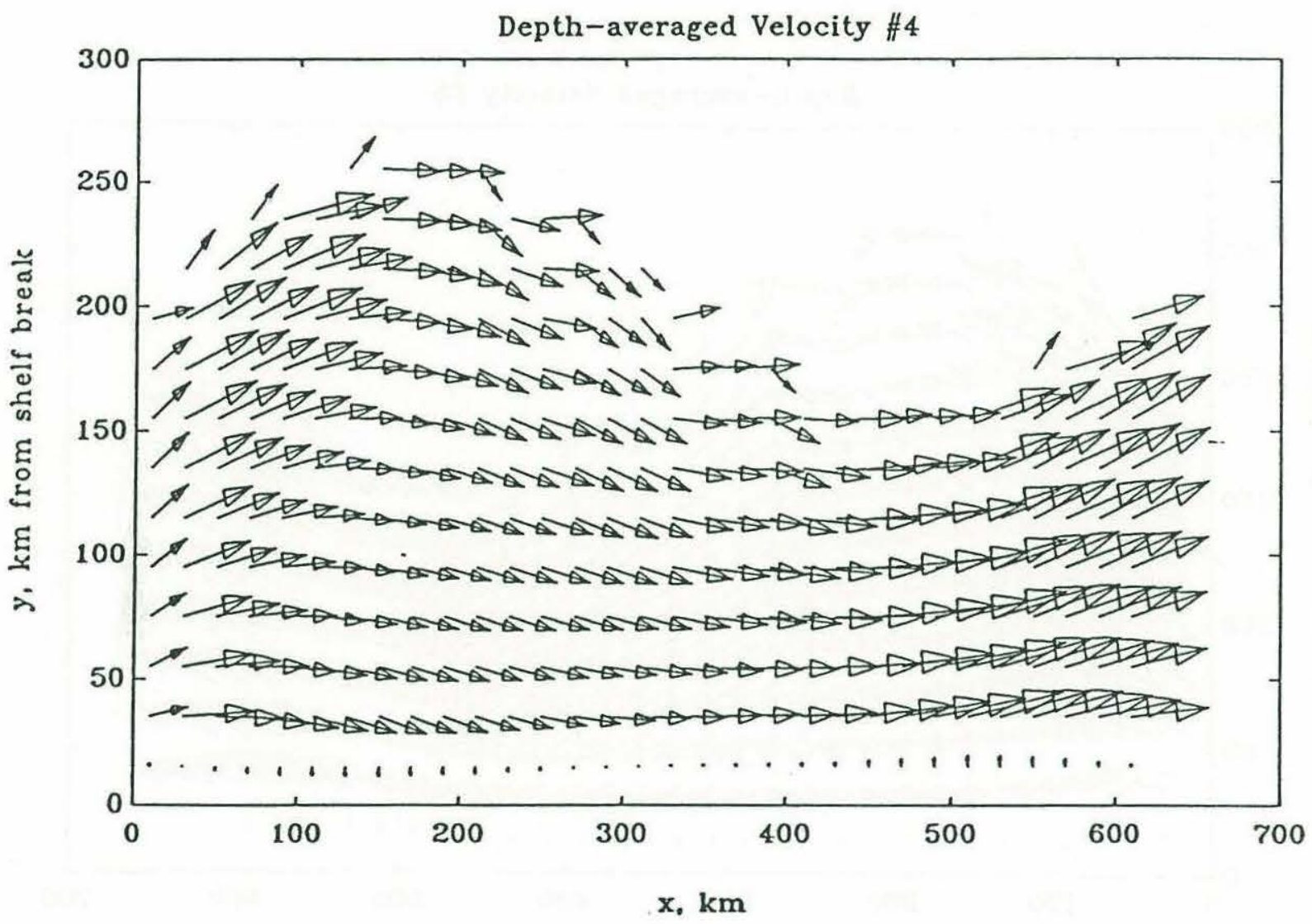

Figure 3-47: Depth-averaged velocity field at 9.6 hrs. Scale: $1 \mathrm{~cm}=60 \mathrm{~cm} / \mathrm{s}$. 


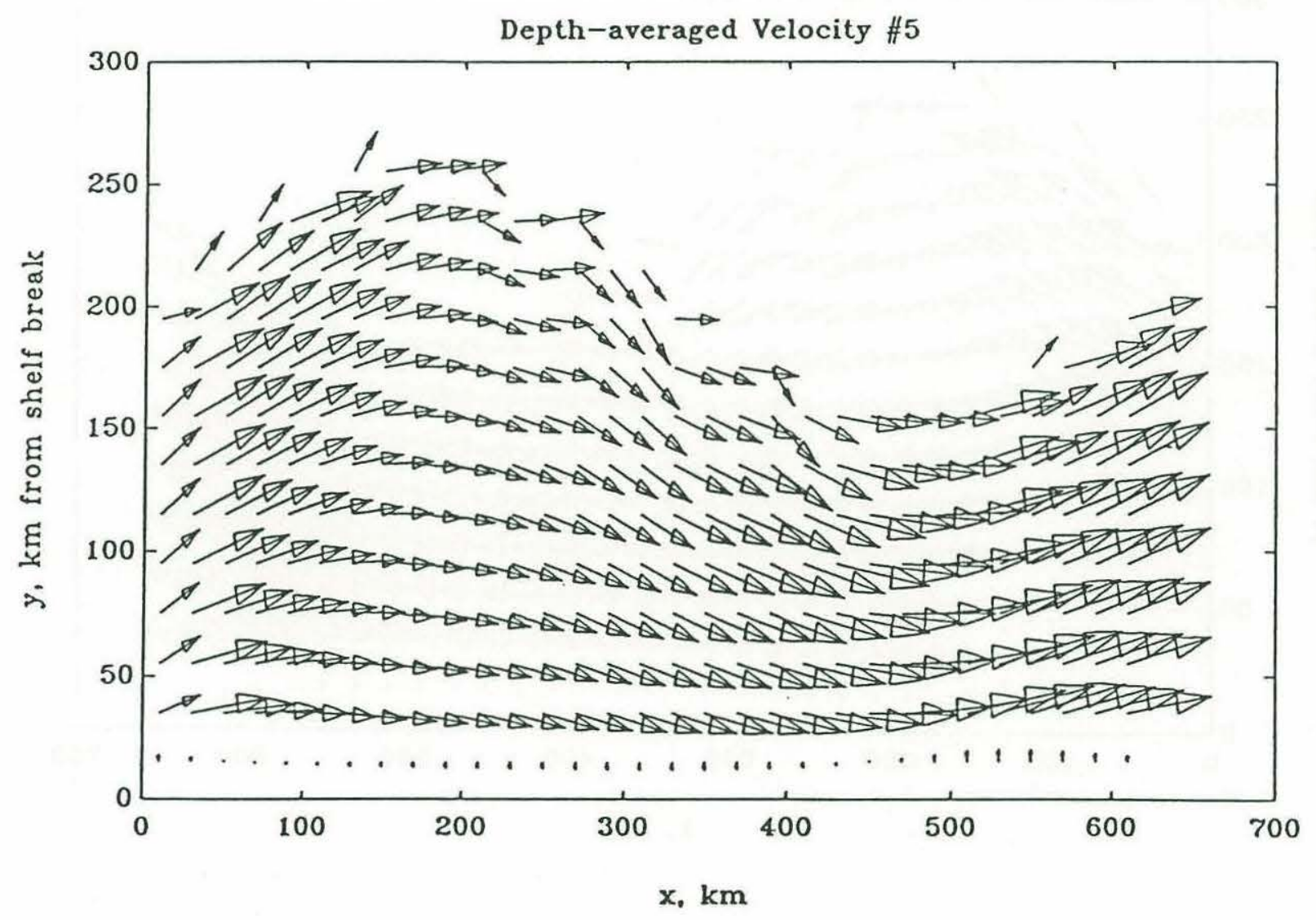

Figure 3-48: Depth-averaged velocity field at $12 \mathrm{hrs}$. Scale: $1 \mathrm{~cm}=60 \mathrm{~cm} / \mathrm{s}$. 
Depth-averaged Velocity \#14

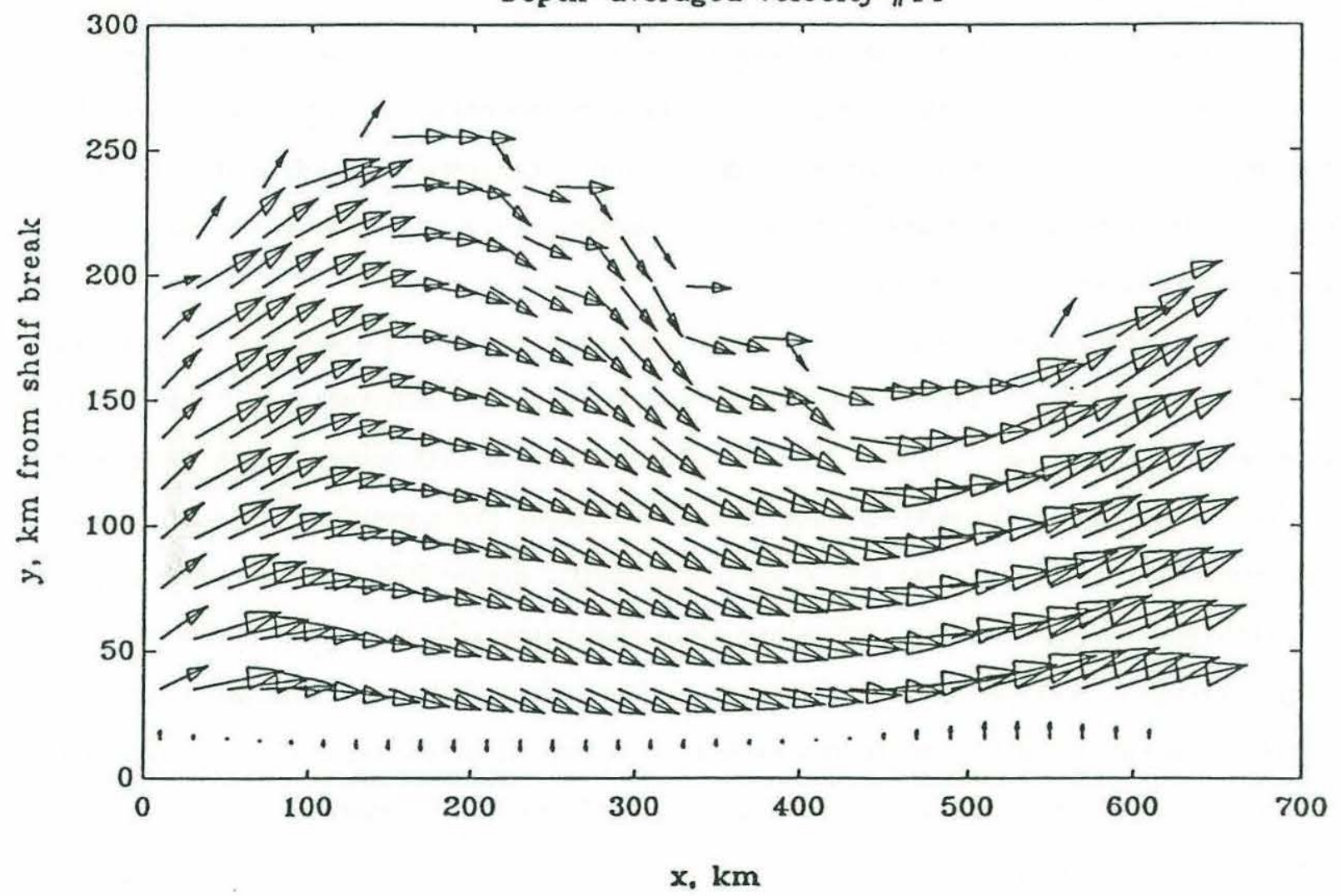

Figure 3-49: Depth-averaged velocity field at steady-state. Scale: $1 \mathrm{~cm}=60 \mathrm{~cm} / \mathrm{s}$. 
pressure gradient farther offshore from the leeward coast is roughly perpendicular to the wind, giving the depression the bowl-like structure visible in the figure. The sea surface is constrained to be 0 along the shelf edge so the contours become straight and parallel to the $x$-axis there.

Figures 3-44-3-49 depict time evolution of the depth-averaged velocity. Initially, the flow moves downwind as is evidenced by the vectors in the lower lefthand corner of the first plot. Once the surface signal propagates out from the coast, however, a large change in speed and direction occurs. The points nearer the coast exhibit an early transition to fast, essentially shore-parallel flow. This behavior is soon adopted by all the points in the domain with a superimposed oscillation due to the seiching motions of the sea surface. At steady-state, the flow is largely shore-parallel with larger velocities where the shelf is narrower. The somewhat strange looking velocities at the first grid point in from the coast are due to both spatially averaging of $U$ and $V$ nodes to yield a velocity vector at the nearest $\zeta$ node and to the fact that the finite difference grid does not smoothly approximate a true sinusoid. These effects disappear a short distance away from the coast.

It is interesting to consider more closely the direction of the steady-state flow. Figure 3-50 shows contours of $(\beta)$. An appropriate question to ask, given this information, is " How nearly shore-parallel is the flow?". If it is shore-parallel everywhere (assuming smooth, sinusoidal coastline and isobaths), the contour lines should be straight and parallel to the $y$-axis. Also, their values should range between $\pm 27.5^{\circ}$ for this geometry. Figure $3-50$ shows that this picture is accurate in a qualitative sense, particularly along those areas of the coastline which are most slowly varying in $x$ (i.e. at the "crest" and in the "trough"). When the coastline is steeper, the picture of flow parallel to sinusoidal isobaths is disrupted by the staircase approximation of the coastline. Offshore, away from the immediate influence of the coastline, the flow is well described as shore-parallel everywhere.

\subsubsection{Bottom stress and drag tensor}

Bottom stress spins up much like depth-averaged velocity. It grows rapidly near the coast and propagates seaward. The stress becomes roughly shore-parallel, and then is modulated by the wavelike adjustment process. At steady-state, the stress is roughly shore-parallel with the largest stresses appearing where the shelf is narrowest (figure 3-51). The stresses near the coast 


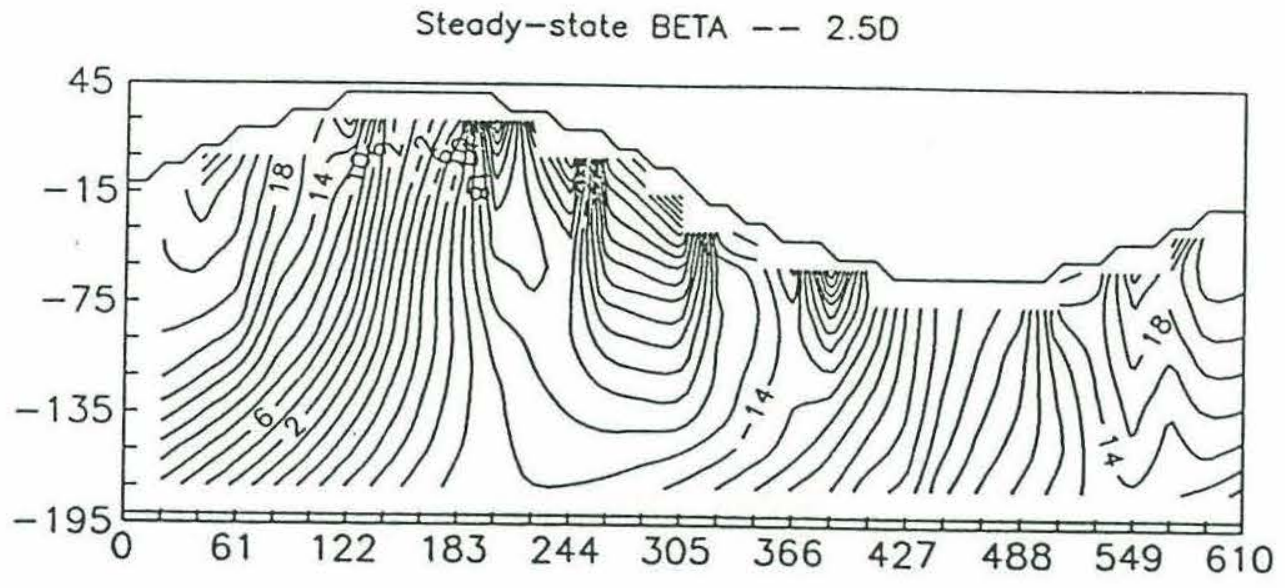

Figure 3-50: Contour plot of $\beta$ at steady-state. Contour interval is $2^{\circ}$.

reflect spatial averaging and the irregularity of the numerical coastline.

The steady-state resistance coefficient (figure 3-52) varies from less than $0.16 \mathrm{~cm} / \mathrm{s}$ to greater than $0.34 \mathrm{~cm} / \mathrm{s}$ and exhibits a great deal of spatial structure. Clearly an assumption of spatially constant $r$ is inappropriate. A bowl-like structure is found in the "trough" where the sea surface was depressed and depth-averaged velocities are at their minimum. Also, the maximum resistance coefficients are found offshore of the "crest" where the depth-averaged velocities are at their maximum. The apparent correlation between resistance coefficient and depth-averaged velocity suggests that it might be interesting to estimate a steady-state field of drag coefficients. Figure 3-53 shows contours of estimated $C_{d}$. The contours are nearlyly sinusoidal in the region of varying depth and roughly constant in the flat area offshore. This indicates that the estimated steady-state drag coefficient field is determined primarily by the quantities $z_{0} / h$ and $u_{* s} / f h$ and not the angle between the wind and the shoreline since the latter is variable along isobaths. The results of sections 3.1 and 3.2 and those of Jenter and Madsen (1989) suggest that the majority of variation in $C_{d}$ is due to variation in $z_{0} / h$ rather than $u_{* s} / f h$.

Another important quantity to be examined is the direction of the bottom stress. Figure 3-54 shows a contour plot of $\alpha$ at steady-state. As with the depth-averaged flow direction, the contour lines are essentially parallel to the $y$-axis where expected. However, they are shifted relative to the contours of $\beta$. This can easily be seen by examining the $0^{\circ}$ contour on the 


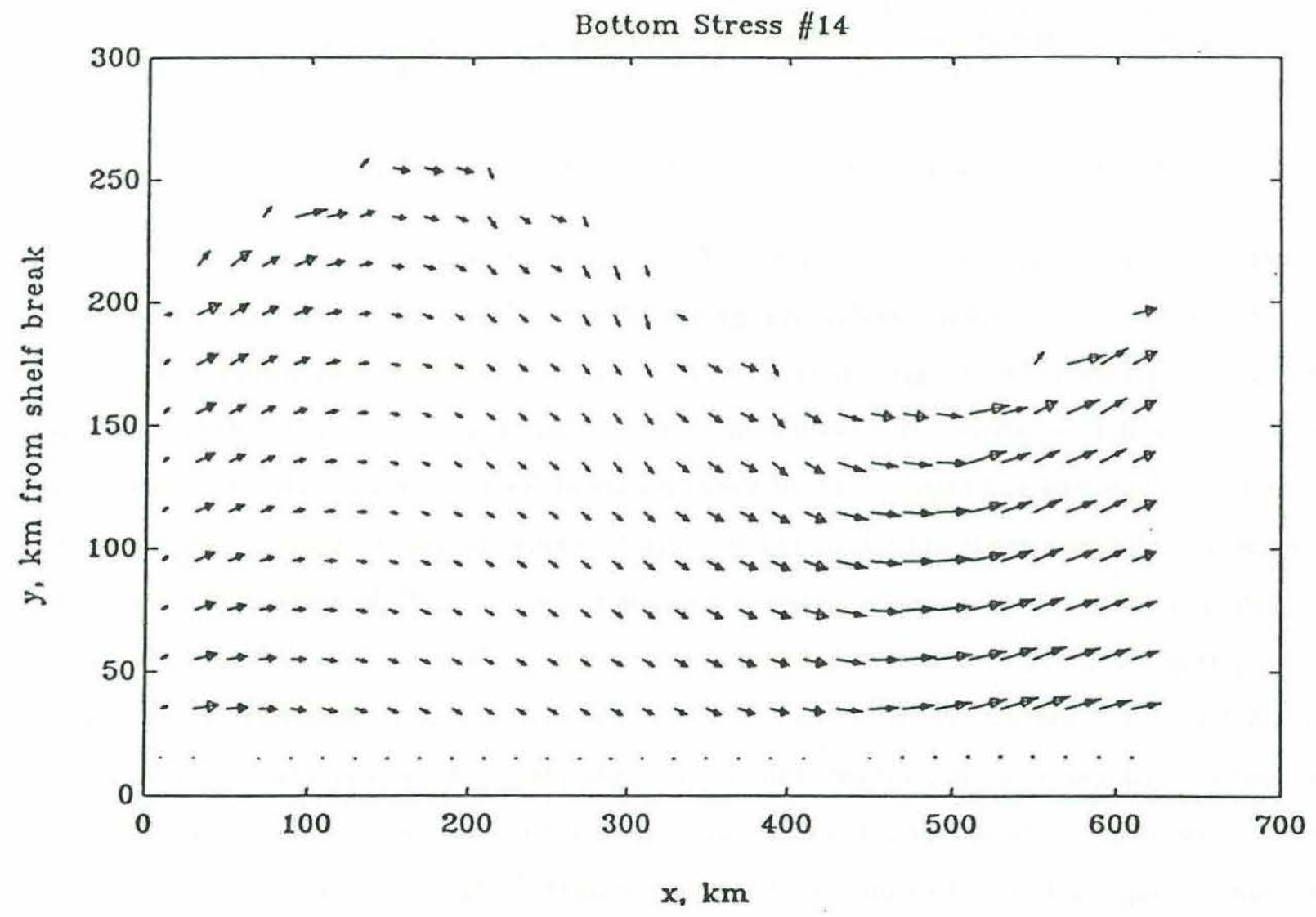

Figure 3-51: Steady-state kinematic bottom stress field. Scale: $1 \mathrm{~cm}=60 \mathrm{~cm}^{2} / \mathrm{s}^{2}$. 


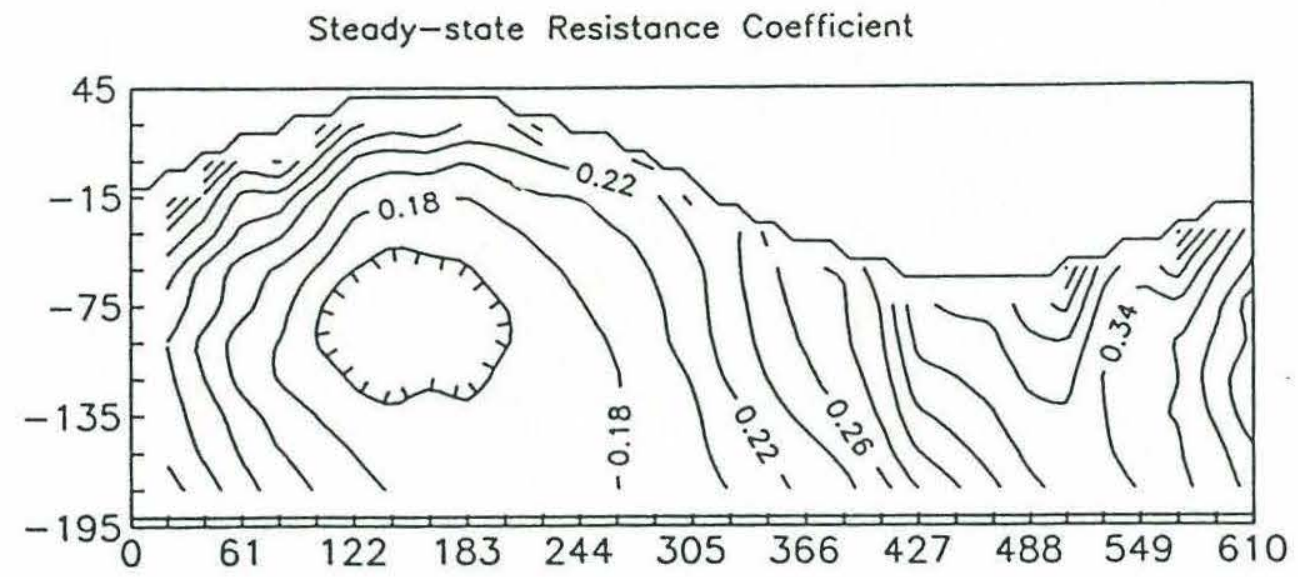

Figure 3-52: Steady-state resistance coefficient.

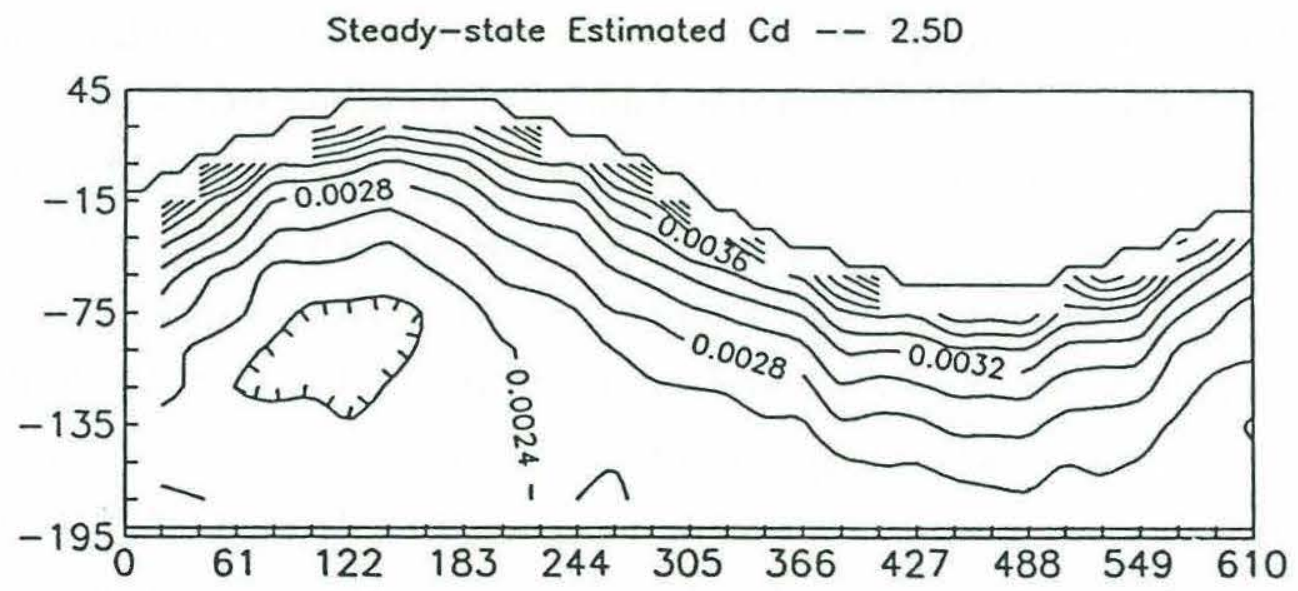

Figure 3-53: Steady-state estimated drag coefficient. 


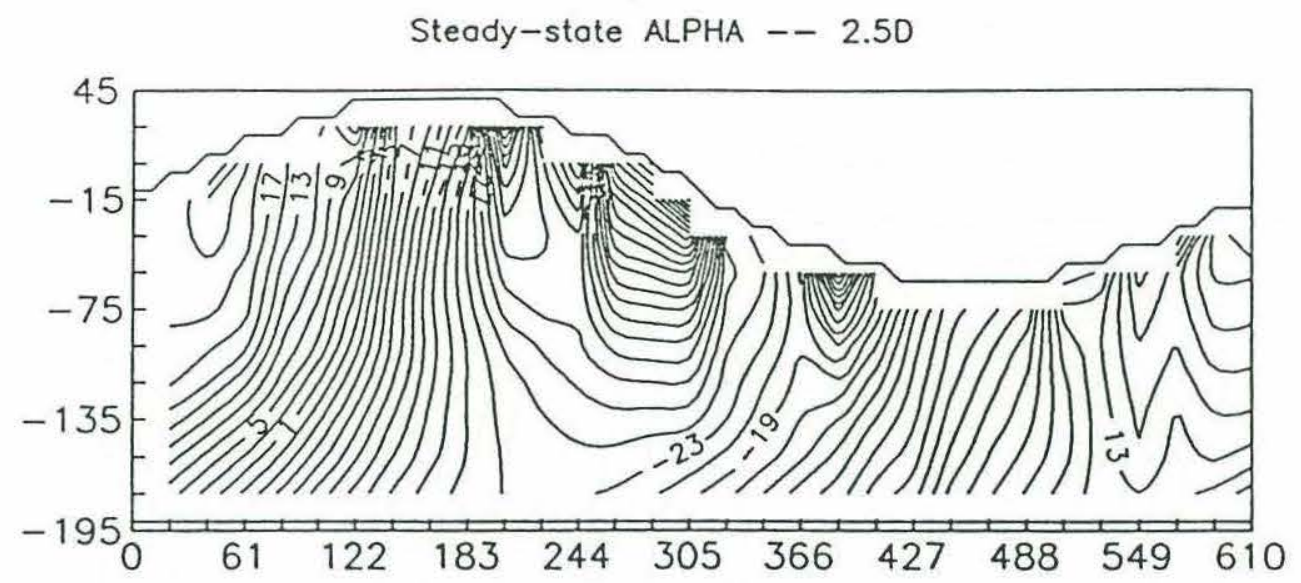

Figure 3-54: Contour plot of $\alpha$ at steady-state. Contour interval is $2^{\circ}$.

lefthand side of each figure. The $\beta=0^{\circ}$ contour (figure 3-50) intersects the coast roughly in the middle of the "trough" as would be expected of truly shore-parallel flow. The $\alpha=0^{\circ}$ contour in figure 3-54, on the other hand, intersects the coast to the left of center in the trough. The $\alpha=-8^{\circ}$ contour bisects the "trough". Therefore, while the approximately straight contours of $\alpha$ parallel to the $y$-axis indicate that the direction of bottom stress is basically a function of the wind angle relative to the coastline, they do not indicate that the bottom stress is shore-parallel.

The difference between figures 3-54 and 3-50 is the steady-state field of $\theta$ shown in figure 3-55. The contours are surprisingly smooth relative to those of $\alpha$ and $\beta$. This is even true one grid point from the coast, indicating that $\theta$ is a fairly robust quantity. It seems to be affected only by the "global" angle between the wind and the coastline rather than the "local" wind angle which varies dramatically from grid point to grid point.

Notice that the range of $\theta$ is between $-1^{\circ}$ and $-16^{\circ}$ indicating that the traditional assumption of $\theta=0$ is not appropriate. Also the fact that it is always negative means the bottom stress is everywhere directed offshore from the depth-averaged flow, which has important implications for near bottom transport. The angular difference can be seen by superimposing the vector plots of steady-state bottom stress and depth-averaged flow. 
Steady-state THETA

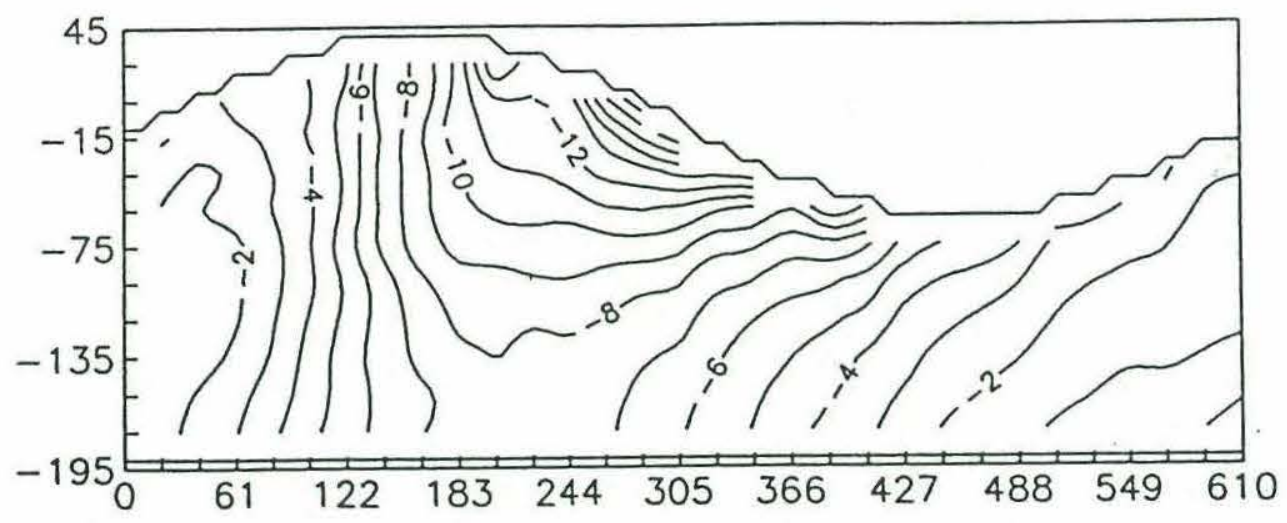

Figure 3-55: Contour plot of $\theta$ at steady-state. Contour interval is $1^{\circ}$.

\subsubsection{Differences betweeen traditional and $2 \frac{1}{2}-\mathrm{D}$ model results}

Sections 3.1 and 3.2 have already illustrated many of the differences between traditional and $2 \frac{1}{2}$-D models. Here a single comparison is made and discussed. The traditional model is given a resistance coefficient, $r=0.25 \mathrm{~cm} / \mathrm{s}$, which is an "eyeball" average of the values in figure $3-52$. Of course, $\theta=0$.

Differences in the surface set-up and depth-averaged velocity fields will clearly be a strong function of the choice of $r$. This is, in fact, common knowledge as many investigators view the resistance coefficient as a tunable parameter by which they can roughly adjust model output fields to agree with measurements. The effectiveness of varying $r$ to alter the steady-state surface height was discussed in detail in section 3.2. The same type of variation in the basic variables for the sinusoidal coastline is not addressed here.

Directional differences in steady-state depth-averaged flow and bottom stress between the two models was not addressed earlier. The former did not exist in the straight coastline cases and the latter was somewhat masked as differences in $\theta$. In the sinusoidal coastline case, both can differ between models. Figure 3-56 shows the steady-state field of $\beta$ from the traditional model ( $\alpha$ and $\beta$ are the same for the DAM). It looks very much like the $\beta$ field from the $2 \frac{1}{2}$ $\mathrm{D}$ model (figure 3-50). The flow is roughly shore-parallel in those regions of the domain not 


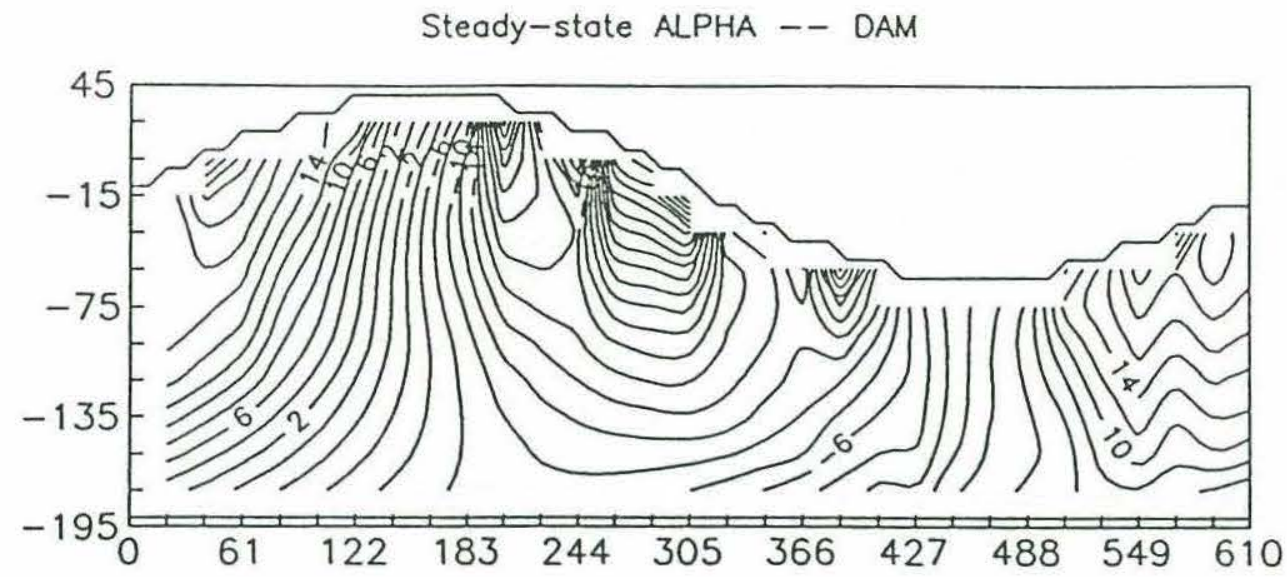

Figure 3-56: Contour plot of $\alpha$ (and, therefore, $\beta$ ) at steady-state from the traditional model. Contour interval is $2^{\circ}$.

affected by the jagged coastline. In fact, the differences between models are shown in figure $3-57$ to be roughly in the range $\pm 5^{\circ}$. Variation in $\beta$ between models is entirely due to the difference in bottom stress formulations.

Figure 3-56 also presents the steady-state bottom stress direction for the traditional model since the flow and stress are constrained to be in the same direction. The traditional model bottom stress is, therefore, more shore-parallel than the $2 \frac{1}{2}$-D model bottom stress. Note that the $0^{\circ}$ contour bisects the "trough" in figure 3-56.

The difference in steady-state $\alpha$ between the two model results is shown in figure 3-58. The values are slightly larger than the differences in $\beta$ and negative for the most part. This means that if the bottom stresses from each model were used to predict near-bed transport direction, the $2 \frac{1}{2}$-D model would predict transport directed more offshore than would the traditional model. Note that the same would be true if the wind were directed at $135^{\circ}$ as the results from section 3.1 presented in table 3.1 showed. 


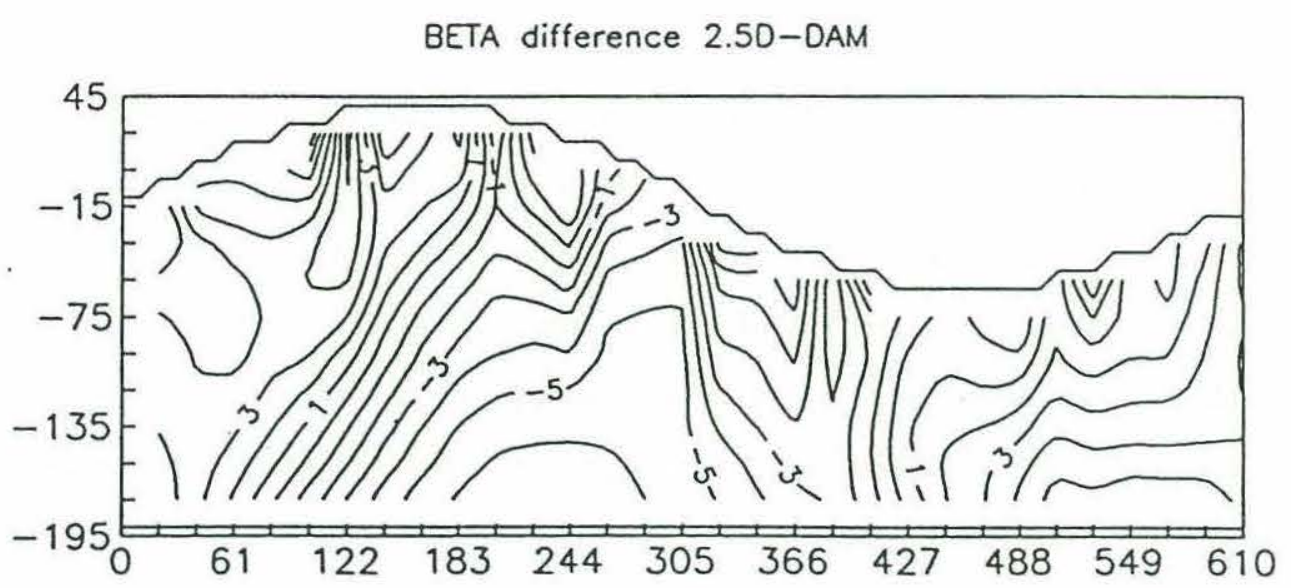

Figure 3-57: Contour plot of the difference between steady-state $\beta$ fields between the $2 \frac{1}{2}$-D and traditional models. Contour interval is $1^{\circ}$.

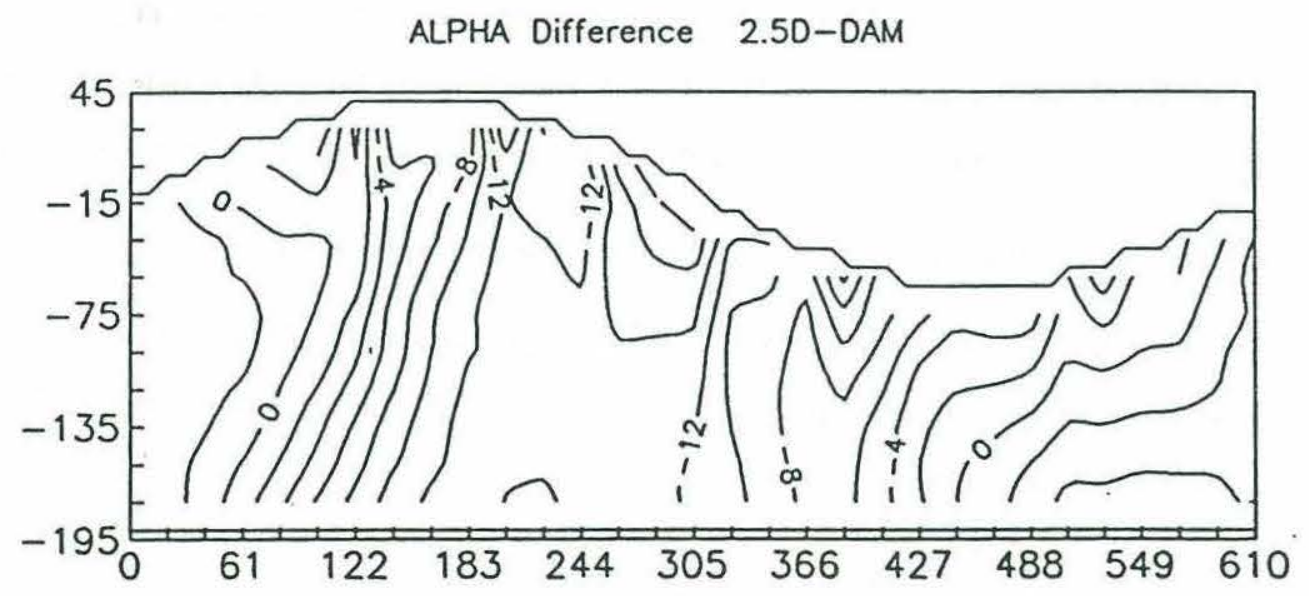

Figure 3-58: Contour plot of the difference between steady-state $\alpha$ fields between the $2 \frac{1}{2}$-D and traditional models. Contour interval is $2^{\circ}$. 


\subsection{Linearly-Varying Depth, Straight Coastline, Rotating Wind Stress}

The previous three sections dealt with transition from rest to steady-state of an open shelf forced by a constant wind stress. It was demonstrated that quantitative differences in both transient and steady-state behavior existed, but that the steady-state differences can be controlled to some extent by allowing for slight modifications of the traditional model such as spatial (but not temporal) variation of the resistance coefficient. In fact, introduction of a correctly chosen spatially-varying drag tensor (using both $r$ and $\theta$ equal to the steady-state $2 \frac{1}{2}$-D model drag tensor) can lead to perfect agreement between the steady-state solutions of the $2 \frac{1}{2}$ - $\mathrm{D}$ and modified traditional model.

Because of this, it is reasonable to ask "Why is a $2 \frac{1}{2}$-D model any better than a modified traditional model?" There are at least three reasons why using a temporally-constant drag tensor field is not as desirable. They are:

- Even though the initial conditions and steady-states might be the same for the two models, the transient behavior will not be the same, as was shown in previous sections.

- It may be very difficult to determine the appropriate drag tensor field a priori. Results like those of Jenter and Madsen (1989) can be used for simple geometries. However, once there is difficulty determining the direction of steady-state flow relative to the wind stress (as in section 3.3), there is no reliable method for estimating the drag tensor field.

- Transient phenomena such as coastal storms may not have a steady-state response.

The first two points were addressed earlier. The third is briefly discussed here. A simple temporally-varying wind stress is applied to an open shelf and the basic variable and drag tensor responses are examined.

\subsubsection{Model domain and wind stress field}

The simplified wind stress field is spatially invariant but rotates uniformly in time. It is described by

$$
\frac{\tau_{s x}}{\rho}=u_{* s}^{2} \cos \frac{2 \pi t}{T}
$$




$$
\frac{\tau_{s y}}{\rho}=u_{* s}^{2} \sin \frac{2 \pi t}{T}
$$

where $T$ is the rotational period of the wind stress. The period is chosen to be $24 \mathrm{hrs}$ giving the stress a diurnal periodicity.

Clearly, this is an idealized wind stress, but it contains the essential features necessary to evaluate the basic relationship between transient wind stress and the associated bottom stress. Actually, the level of complexity, is similar to many studies of "storm"-generated coastal circulation (e.g. Chao, 1981; Carton, 1984; and Heaps et al., 1988). There have been more realistic wind stress fields employed for studying wind-driven coastal responses, but these have usually been aimed at studying a particular geographic area's response or a specific type of meteorological event (e.g. Jelesnianski, 1965; Beardsley and Haidvogel, 1981 and Flather and Khandker, 1987).

The magnitude of the wind stress corresponds to $30 \mathrm{~m} / \mathrm{s}$ wind speed as used in earlier scenarios. Its direction, however, is linearly variable in time rather than constant. The basin geometry is identical to that in section 3.2 with a straight coastline and a linearly-sloping bottom. The bottom roughness length is again given by $z_{0}=1 \mathrm{~cm}$.

Because of the equivalent geometries the diffusion and wave time scales are the same as those in sections 3.2 and 3.3, and that the wind stress period is long in comparison. This means that the water column has time to respond to small changes in wind direction. If the period of the wind stress were shorter than the diffusion time scale, the water column could not be expected to respond appropriately. As discussed in chapter 2, the 1-D model acts as a low-pass filter, only communicating slowly-varying information to the bottom.

\subsubsection{Sea surface height and depth-averaged velocity}

The response of the shelf to periodic forcing is in many ways different from the scenarios presented earlier. In particular, the flow field does not reach a true steady-state (i.e. one where there is no temporal change within the domain after large times). Rather, it reaches a situation where the motion is periodic. This is clearly illustrated in figure $3-59 a-c$ where time series of surface height, alongshore velocity and cross-shore velocity are shown at the same onshore and offshore points as defined in section 3.2. 

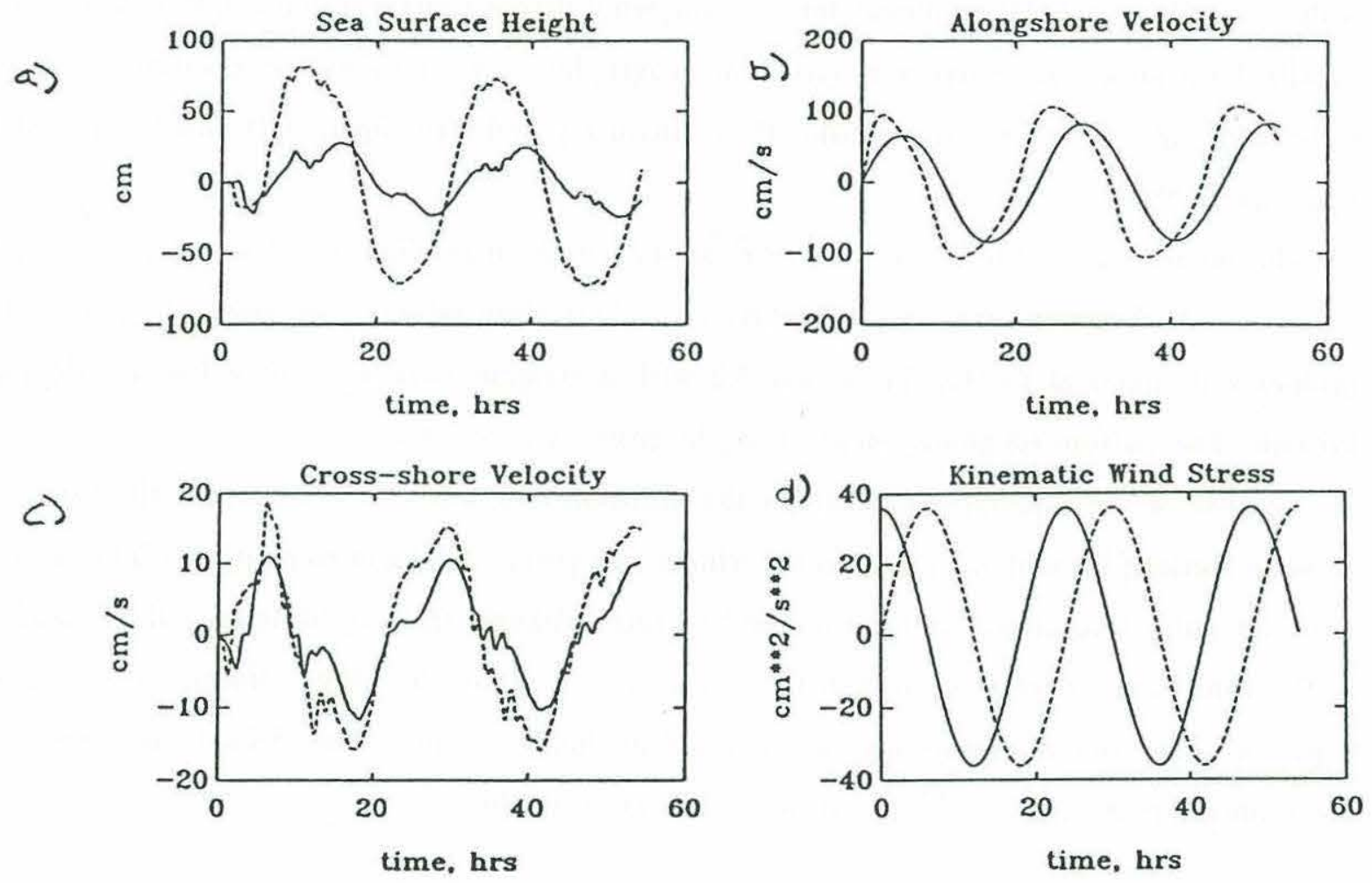

Figure 3-59: Time series of a) surface height, b) alongshore depth-averaged velocity, c) crossshore depth-averaged velocity at the offshore (solid line) and onshore (dashed line) points and d) alongshore (solid line) and cross-shore (dashed line) kinematic wind stress. 
The sea surface signal behaves as would be expected. The larger response is onshore away from the pinned shelf edge. Also, the onshore point responds first as the signal propagates out from the coast. Consequently, the onshore response leads the offshore response. This can be seen in the alongshore velocity signal too.

The initial response of the sea surface at each point shows a very small rise followed by a more pronounced drop because the wind direction starts out at $0^{\circ}$ and rotates toward the shore (figure 3-59d). There is a small bulge pushed up against the shore during the first few time steps. This creates the slight positive signal. Shortly thereafter, however, a positive alongshore flow develops sufficiently to move water away from the coast through Coriolis acceleration, thereby causing the dip. The motion is nearly periodic from that time on.

There is noticable high frequency motion in figures $3-59 \mathrm{a}$ and $3-59 \mathrm{c}$. This is due to small alongshore pressure gradients generated each time the wind is exactly perpendicular to the shore. As explained earlier, the equations of motion are degenerate when the wind is straight onshore. However, longer model runs than those presented reveal that the high frequency motions do not grow from one period to the next. Even though the motions are generated when the wind is cross-shore, they are most strongly forced when it is alongshore. In figures $3-59 \mathrm{a}$ and $3-59 \mathrm{c}$, times of maximum high frequency motion correspond to times when the wind is directly alongshore. They are sufficiently damped as the wind rotates to render them stable over long times. The magnitude of the small alongshore pressure gradients ranges from $1 \times 10^{-4}$ $\mathrm{cm} / \mathrm{s}^{2}$ at generation to $1 \times 10^{-3} \mathrm{~cm} / \mathrm{s}^{2}$ when they are forced alongshore. This corresponds to alongshore height differences between grid points of 0.1 and $1 \mathrm{~cm}$, which are very small.

Figure 3-60 shows contours of surface height in the $y-t$ plane. The basic periodic behavior is immediately apparent. There is a slight indication of the signal propagating outward from the shore as is evidenced by the left side of each set-up or set-down period being cusplike in shape. Not surprisingly, a cusplike behavior was also seen in section 3.2 (figure 3-26). There is a difference, however, in that the wind stress here is periodic thereby coupling the sea surface response to a particular frequency. Therefore, the oscillatory behavior is a combination of free and forced waves.

The initial positive signal and subsequent drop propagating out from the shore are seen on the left side of figure 3-60. These are also readily visible in figure 3-61 which shows the sea 
Seo Surfoce Height, $\mathrm{cm}$

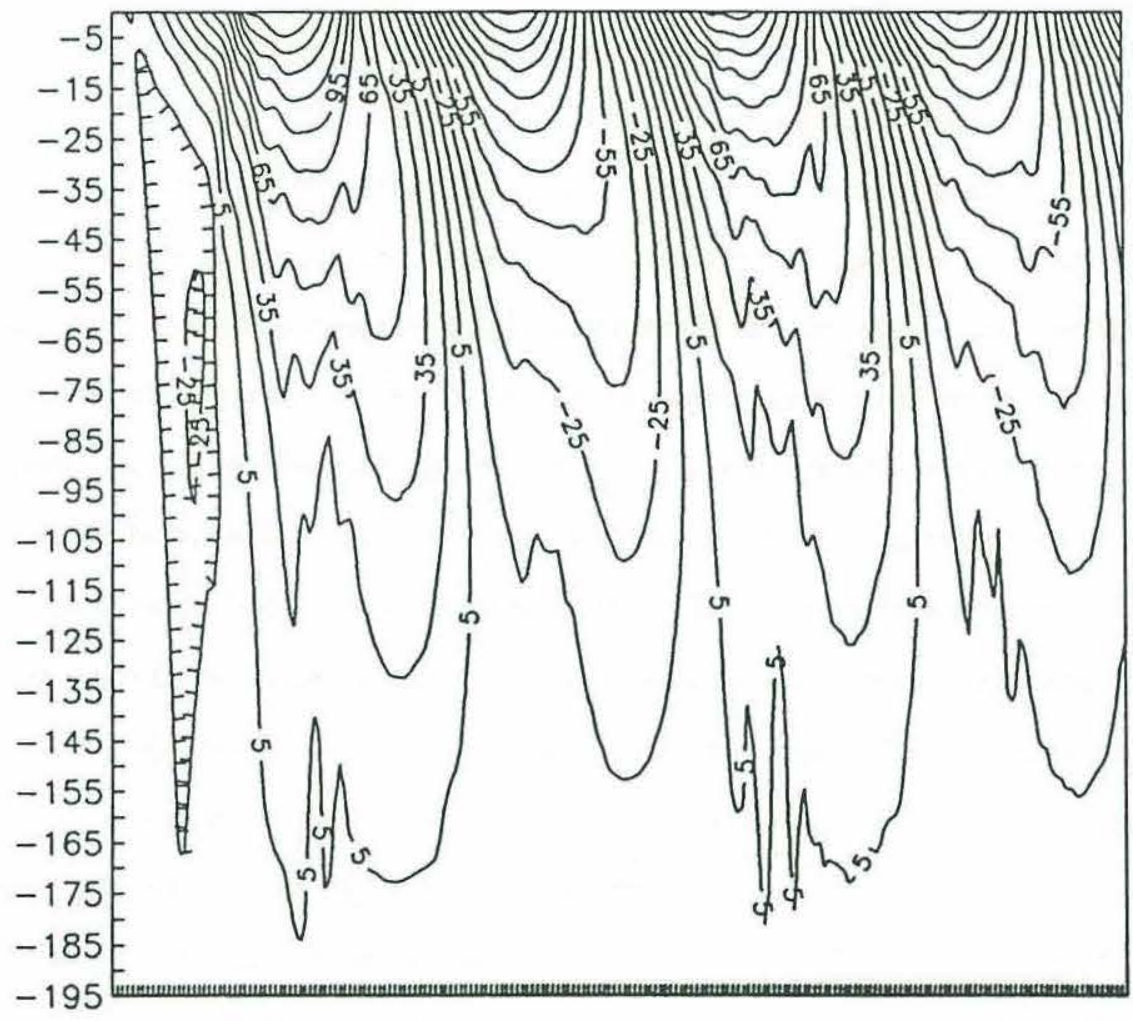

Figure 3-60: Contour plot of surface height on the $y-t$ plane. Tic marc spacing is $5 \Delta t$ and $0.5 \Delta x$ on the horizontal and vertical axes, respectively. Time ranges from 0 to $50 \mathrm{hrs}$. 
surface behavior in three dimensions. The high frequency part of the signal is seen as ripples along the "peaks" and "valleys" in figure 3-61. It is somewhat difficult to tell from the figure, but the high frequency signal is generated locally everywhere across the shelf at the same time. In other words, it does not have the signature of a disturbance created at a particular cross-shelf location and propagated across the shelf.

The alongshore velocity signal in figure $3-59 \mathrm{~b}$ shows some interesting behavior when compared with the results for the linear depth constant wind stress case. Both scenarios have the same geometry and wind stress magnitude. The only difference is that the wind direction is constant in section 3.2 and variable here. The alongshore velocity in the rotating wind case has a larger amplitude onshore than offshore. The opposite is true at steady-state for the constant wind stress. For short times, however, the constant wind develops larger alongshore flow at the onshore point. This was attributed to the more rapid response time in shallow water. The same feature appears in figure $3-59 \mathrm{~b}$. The shallow water response time is short enough so that the signal behaves more like a succession of steady-states while the deeper part of the domain does not respond fast enough. In other words, the offshore point has not approached steady-state by the time the wind stress changes directions causing the flow to seek a new steady-state.

The notion of slow response offshore is supported by recalling the shallow and intermediate spin-up times from section 3.1. They were $5 \mathrm{hrs}$ and $10 \mathrm{hrs}$, respectively. Clearly, a water column that takes $10 \mathrm{hrs}$ to spin-up is not able to "keep up" with a wind stress that varies with a period of $24 \mathrm{hrs}$. A water column which takes $5 \mathrm{hrs}$ to spin-up does a better (but not perfect) job of following the wind stress.

It is difficult to say whether the amplitude of the alongshore flow oscillation at the inshore point in figure $3-59 \mathrm{~b}$ is equal to the maximum steady-state alongshore flow due to a constant wind stress, since not all wind directions were examined in section 3.2. However, it is larger than the alongshore velocity for a $45^{\circ}$ wind. The situation is different at the offshore point. The amplitude there is less than the steady-state velocity maximum for a constant wind, since it is less than the constant $45^{\circ}$ wind result. This is another indication that the flow is not capable of responding fast enough at the offshore point.

There is another interesting phenomenon in the alongshore velocity signal. The time series is not as sinusoidal at the onshore point as it is at the offshore point. The main reason for the 


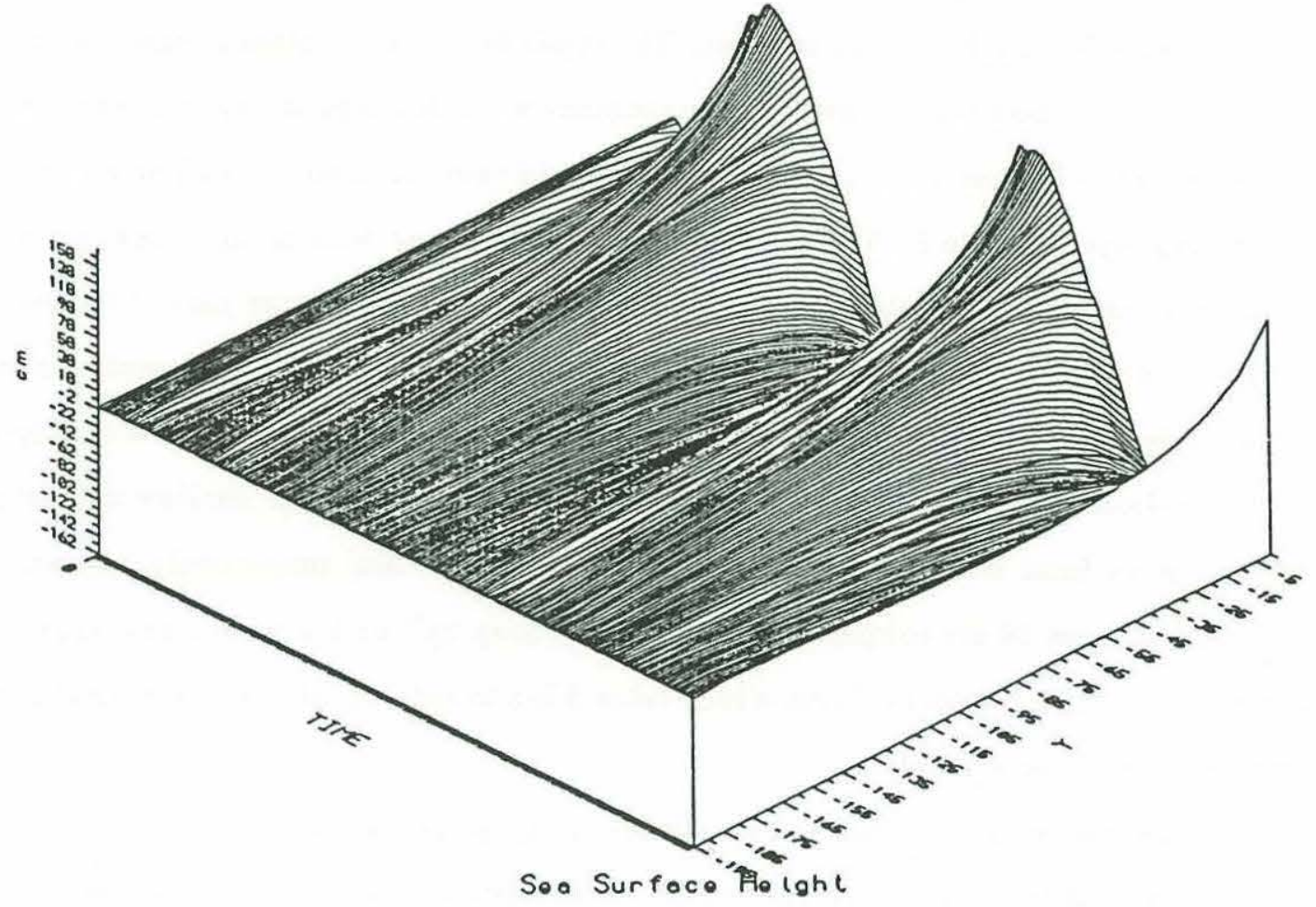

Figure 3-61: 3 -D plot of surface height on the $y$ - $t$ plane. Tic marc spacing is $5 \Delta t$ and $0.5 \Delta x$ on the time and $y$ axes, respectively. Time ranges from 0 to $50 \mathrm{hrs}$. 
asymmetic crests and troughs in the signal is that sometimes the alongshore wind stress and cross-shore velocity are in phase causing $\tau_{s x} / \rho$ and $f V$ to have the same sign, and sometimes they are out of phase causing opposite signs. The former yields large values of $\partial \vec{U} / \partial t$ and, therefore, steeper variation in the signal. The effect does not show up offshore because the phase relationship between the wind stress and cross-shore velocity is different there.

\subsubsection{Drag tensor}

Figure 3-62 depicts the time-dependent behavior of the resistance coefficient at the onshore and offshore points. It is clearly not constant. Both points start out at 0 when the domain is at rest. The onshore point grows most rapidly at first since the depth-averaged flow develops fastest there. After the initial spin-up, the resistance coefficient varies periodically with twice the frequency of the wind stress. This is because the resistance coefficient is positive definite, being determined by the bottom stress and depth-averaged velocity magnitudes. Each of which has two maxima during one period of the wind stress.

Notice that the resistance coefficient displays the high frequency variation seen earlier in the sea surface signal and cross-shore velocity. This is understandable since the bottom stress determined by the DRM is a function of the pressure gradients in the DAM. Because the high frequency motions indicate the presence of small alongshore pressure gradients, the DRM bottom stress and the resistance coefficient are affected.

In addition to the high frequency motion, the resistance coefficient offshore shows a large spike during each period, which may be explained with time series of bottom stress and depthaveraged velocity magnitude (figure $3-63$ ). When the depth-averaged speed drops to nearly zero, the bottom stress magnitude is small but slightly lagging the speed. Consequently, the resistance coefficient is large before dropping to almost zero as the bottom stress becomes small and the depth-averaged speed begins to grow. The "spike" phenomenon was also seen in figure 3-30.

The fact that the resistance coefficient is not constant in time leads to the question "Would a drag coefficient be any steadier?" This was addressed to some extent in section 3.1 where it was shown that the steady-state drag coefficient was more predictable than the steady-state resistance coefficient. However, both quantities showed significant time-dependence. The same 


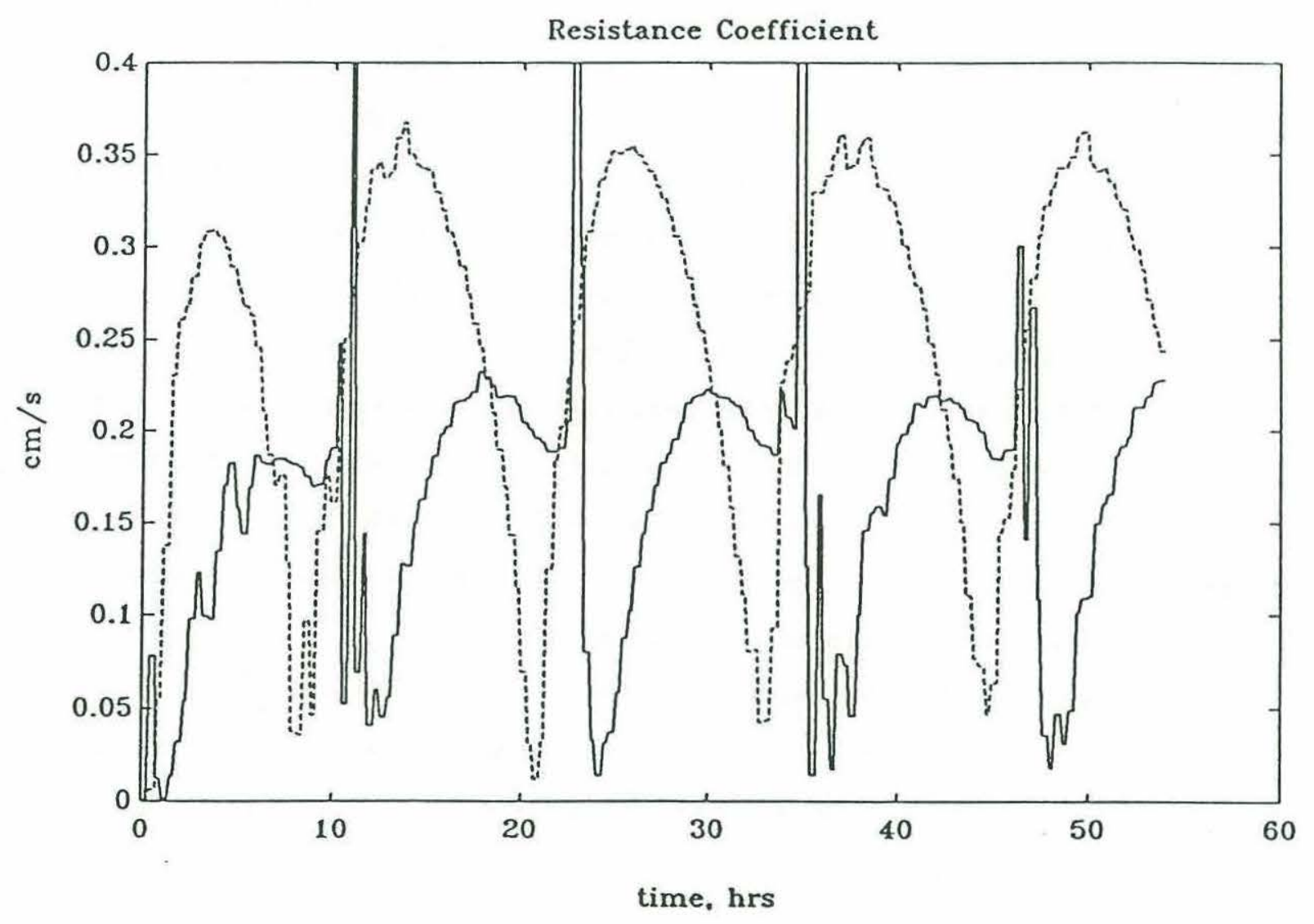

Figure 3-62: Time series of resistance coefficient at the offshore (solid line) and onshore (dashed line) points. 

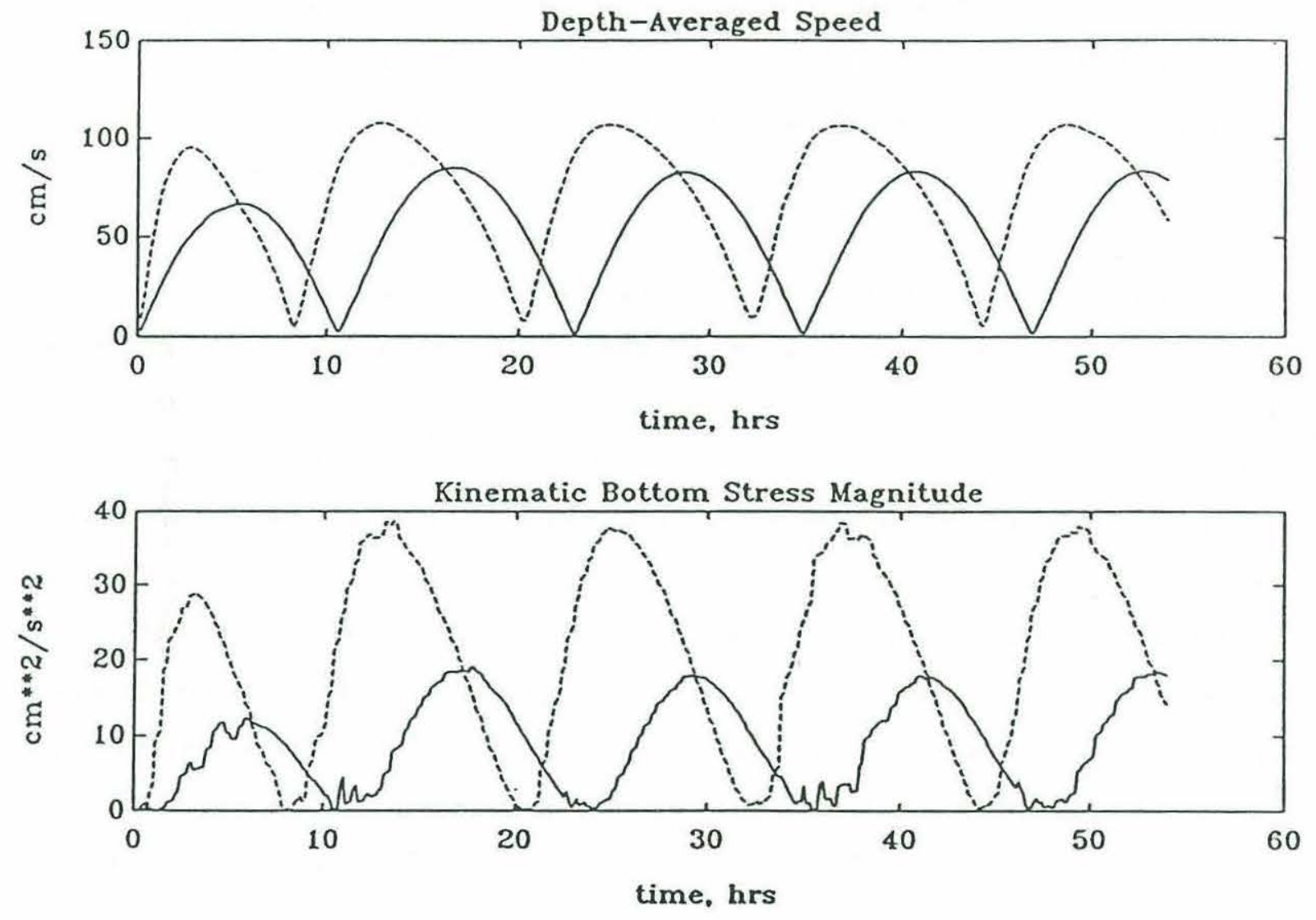

Figure 3-63: Time series of a) depth-averaged velocity magnitude and b) kinematic bottom stress at the offshore point (solid line) and the onshore point (dashed line). 
is true for the oscillatory wind stress scenario. Figure 3-64 shows the estimated drag coefficient which exhibits as much temporal variability as the resistance coefficient 3-62.

As it was for the resistance coefficient, the frequency of variation in $C_{d}$ is twice that of the wind stress. However, each oscillation of $C_{d}$ is antisymmetric about the line $C_{d} \approx .0035$ and a line half way between each peak. Notice that the resistance coefficient increases and decreases nearly symmetrically (disregarding the peaks at the offshore point). This occurs because there are times when the velocity is increasing (decreasing) more slowly than the stress while at the same time the square of the velocity is increasing (decreasing) more rapidly.

Figure 3-65 shows the variation of $\theta$ with time, which is clearly not constant. Disregarding the wrap-around spikes, there are substantial periods during which $\theta$ varies by at least $20^{\circ}$ or $30^{\circ}$. It is hard to imagine that a traditional drag law would be appropriate in light of this variation.

The temporal behavior of $\theta$ is expected based on previous results. Table 3.1 showed that for the flat bottom shallow case, the steady-state value of $\theta$ varied substantially with wind direction. In fact the range of values in the table closely corresponds to the range in the figure. Note also that $\theta$ rotated clockwise in table 3.1 as the wind direction rotated clockwise, a feature of figure 3-65 as well. The unstable behavior described in section 3.1 for $90^{\circ}$ wind angles corresponds to the wrap around here.

Interestingly, the values in figure $3-65$ are " $90^{\circ}$ out of phase" with those in table 3.1 . Here the wrap around occurs when the wind is nearly shore-parallel. This happens because the bottom stress and depth-averaged velocity are nearly zero at those times, making $\theta$ extremely sensitive to small changes in the bottom stress or depth-averaged velocity. For the steady wind situation, depth-averaged velocities were nearly zero when the wind was perpendicular to the shore.

Clearly, the large range of $\theta$ and its complicated phase structure make a priori determination unlikely. This is merely one of many strong indications presented in this thesis that traditional drag laws must be modified to include temporal, spatial and directional effects in order to reproduce correctly the relationship between depth-averaged velocity and bottom stress. 


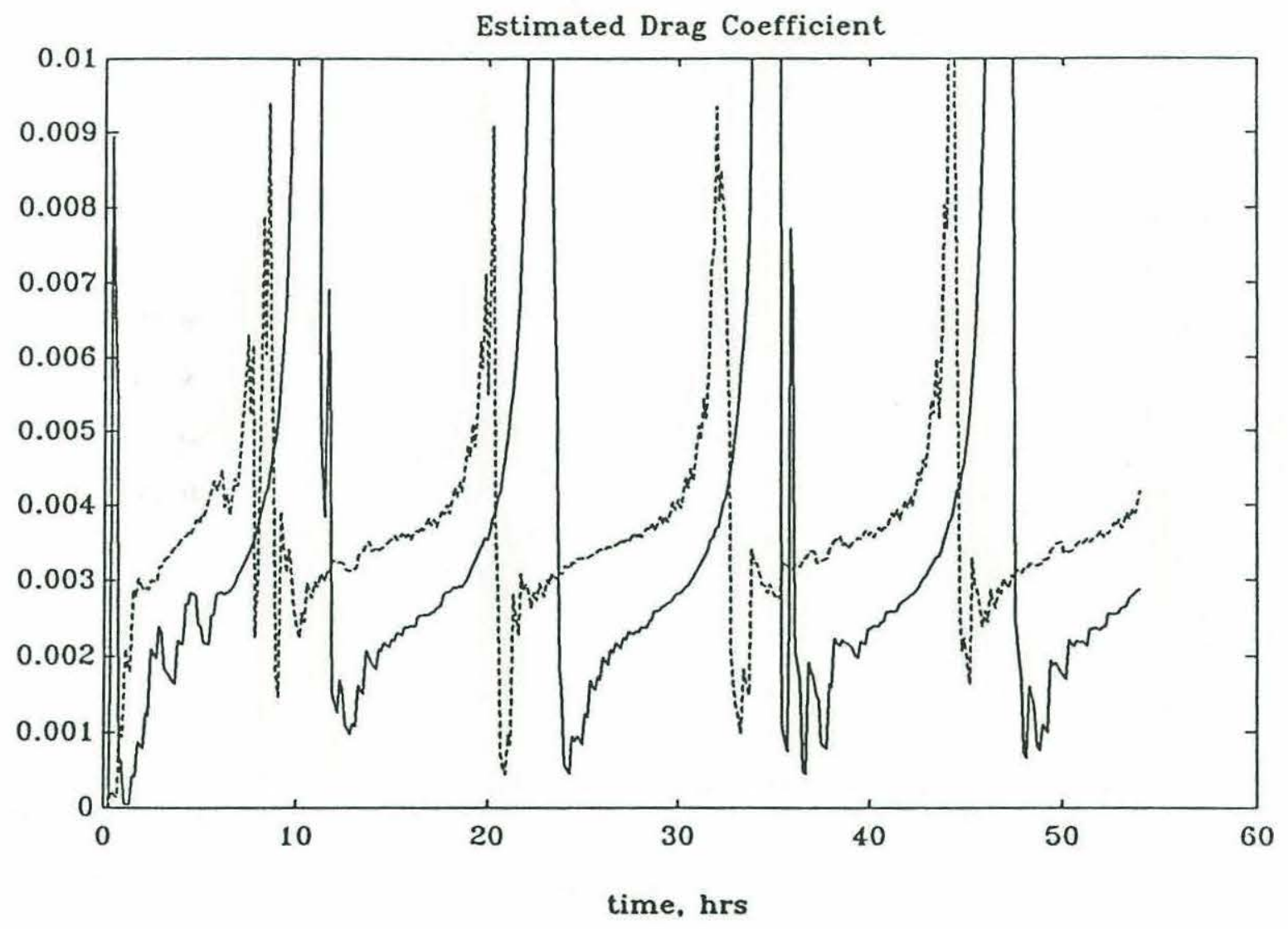

Figure 3-64: Time series of estimated drag coefficient at the offshore point (solid line) and the onshore point (dashed line). 


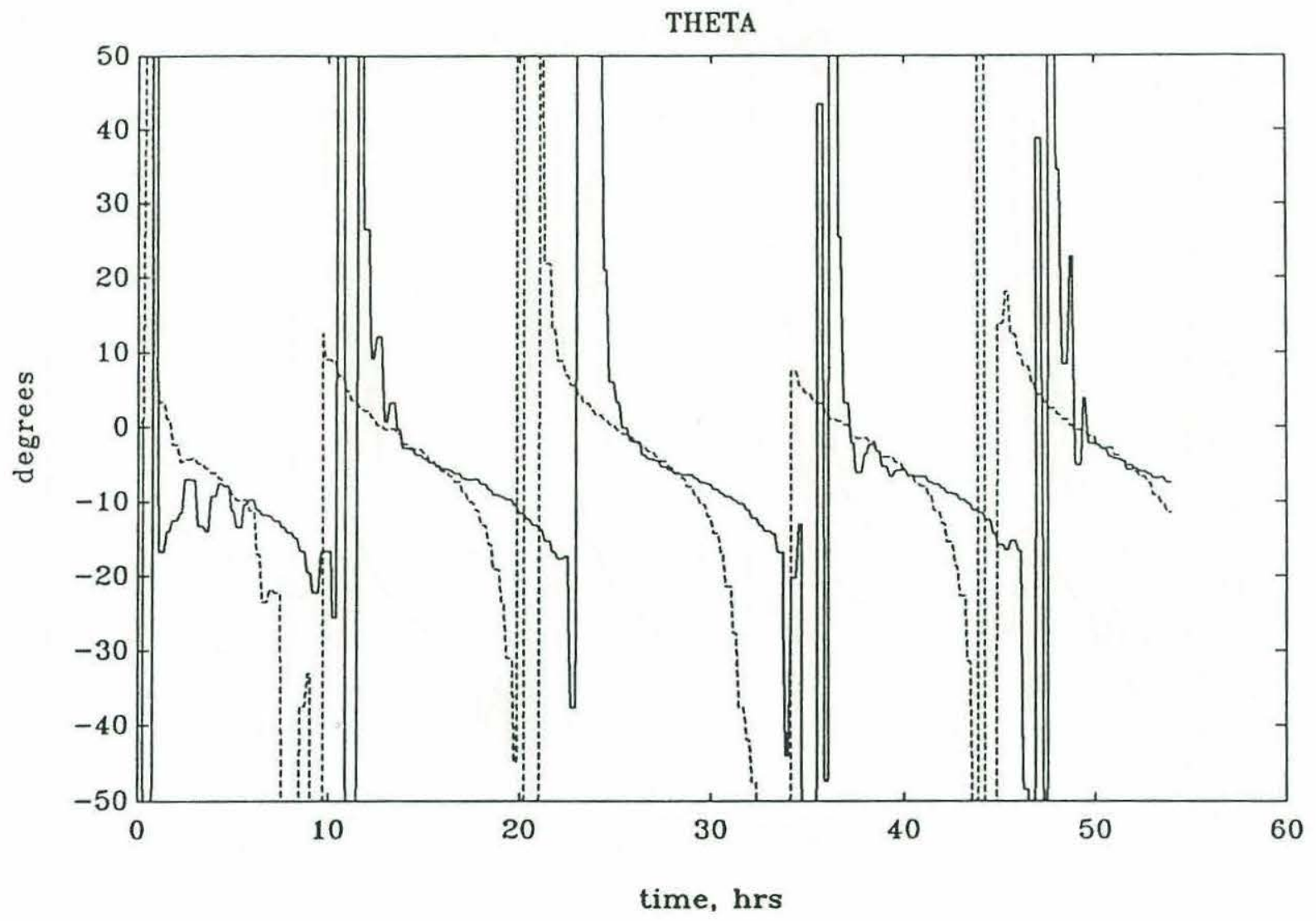

Figure 3-65: Time series of $\theta$ at the offshore point (solid line) and the onshore point (dashed line). 


\subsubsection{Differences between traditional and $2 \frac{1}{2}-\mathrm{D}$ model results}

One of the most interesting aspects of this scenario is the difference in response times at the onshore and offshore points. This leads to differences in response magnitude between steady and periodic wind cases and, in some parts of the domain, to an asymmetry in the perioidic alongshore velocity signal. The key feature is the phase relationship between the wind stress and the flow field. As previous results demonstrated, the temporal response of any domain is a strong function of the drag law. Therefore, one would expect that a traditional drag law would significantly alter the phase relationship between the wind stress and flow field in this scenario.

Figure 3-66 shows time series of alongshore velocity at the onshore and offshore points for the $2 \frac{1}{2}$-D model and for a constant $r$ model with $r=.25 \mathrm{~cm} / \mathrm{s}$, roughly halfway between

peak values for the onshore and offshore points in the $2 \frac{1}{2}$ - $\mathrm{D}$ model. As expected, the phase relationship between the wind stress and flow field is significantly altered. The asymmetry in the signal at the onshore point is gone. Peaks for the constant $r$ case lag those of the $2 \frac{1}{2}$-D model. This is the same phenomenon seen in section 3.1 when the constant $r$ case responded more slowly to a steady wind stress.

Not surprisingly, the magnitude of the alongshore velocity is also different between the models. At the onshore point, the traditional model over-predicts the velocity because the resistance coefficient is too small. At the offshore point, the velocity is too small because $r$ is too large. Clearly, the flow field is sensitive to the choice of drag law in this scenario, as it was in previous sections. 


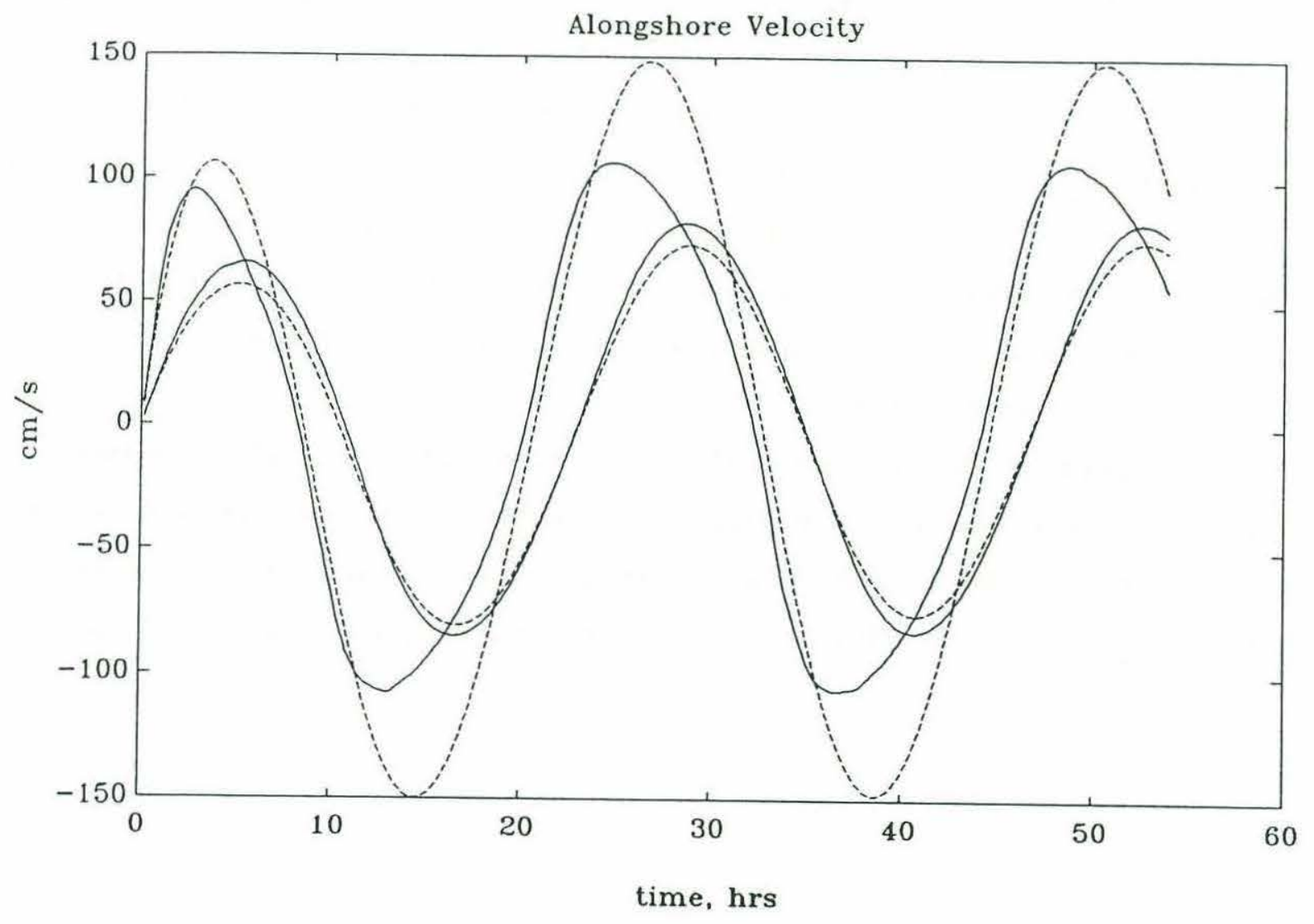

Figure 3-66: Time series of alongshore velocity for the $2 \frac{1}{2}$-D model (solid lines) and for the constant $r$ model (dashed lines) at the onshore point (large amplitude signal) and at the offshore point (small amplitude signal). 


\section{Chapter 4}

\section{Summary and Conclusions}

The main objective of this work was to develop an improved means of specifying bottom stress in wind-driven depth-averaged circulation models. The result is a so-called $2 \frac{1}{2}$-D model which uses a simplified 1-D formulation to provide a drag tensor at each grid point of a standard depth-averaged model.

The drag tensor is used to scale and rotate the depth-averaged velocity to yield a bottom stress. Rotation eliminates the restrictive assumption of traditional depth-averaged models that depth-averaged velocity and bottom stress are in the same direction.

The results presented in this thesis are intended to show three things. They are

- the role of bottom stress in wind-driven coastal flows

- the variation of the drag tensor in these situations

- a comparison between the $2 \frac{1}{2}$-Dimensional model results and those of a traditional depthaveraged model.

The major findings relevant to each are summarized below.

\subsection{The Role of Bottom Stress}

Perhaps the most important role of bottom stress is in setting the response time scale of a particular domain to a particular wind forcing. The two frictional parameters which determine the response time were shown to be 
- $u_{* s} / f h$ - where $u_{* s}^{2}$ is the magnitude of the kinematic wind stress, $f$ is the Coriolis parameter and $h$ is the water depth.

- $z_{0} / h$-where $z_{0}$ is the bottom roughness length.

The typical range of $u_{* s} / f h$ for flows of interest in this thesis is roughly $5-100$, while the range of $z_{0} / h$ is $10^{-3}-10^{-9}$. The latter includes large "apparent roughnesses" due to wave-current interaction and bedforms.

There are actually two time scales in the wind-driven response. The first corresponds to a smoothly-varying overall approach to steady-state, and the second to the decay of higher frequency seiches that propagate back and forth across the shelf. Both are functions of $u_{* s} / f h$ and $z_{0} / h$. As was shown in section 3.1 , the two time scales are roughly the same in domains of constant depth. The results yielded times of 5,10 , and $20 \mathrm{hrs}$ for $u_{* s} / f h=30,10$, and 5 , respectively. Since the depth was constant in all cases, $z_{0} / h$ was always the same. The different time scales were, therefore, entirely due to different values of $u_{* s} / f h$. The larger $u_{* s} / f h$, the shorter the time scale.

The two time scales are different in domains with variable depth. The overall adjustment time for any particular point was found to be a function of the local values of $u_{* s} / f h$ and $z_{0} / h$, while the wave decay time was determined by more "global" values. The non-local effect makes sense because the waves propagate around the domain spending time in all water depths. Consequently, long decay times, comparable to those in deeper water, can be experienced in shallow water. This was demonstrated in section 3.2 .

For time-varying forcing, the ability of any particular point in a domain to respond to that forcing is a function of its overall spin-up time. If the spin-up time is short relative to the time scale of the forcing then the flow behaves somewhat like a progression of individual steadystates. This was the case at the onshore point in section 3.4. If the spin-up time scale is not short enough then the flow response is diminished because it cannot "keep up" with the forcing. The offshore point in section 3.4 was a good example of this.

In addition to determining time scales, bottom stress also significantly affects the magnitude of response. Seiche and steady-state magnitudes are related to $u_{* s} / f h$ in much the same way that the decay and overall spin-up times were. In domains of constant depth, the magnitudes are larger for larger values of $u_{* s} / f h$. In domains of variable depth, the steady-state magnitude 
is still a function of the local value of $u_{* s} / f h$, but the wave amplitude is determined by the value of $u_{* s} / f h$ in the region of its generation.

The results of sections 3.1 showed that the bottom stress plays somewhat different roles in the alongshore and cross-shore momentum balances. In the alongshore direction, there must be a Couette balance at steady-state in which the bottom stress and wind stress are equal and opposite. In the cross-shore direction, the primary steady-state balance is between the wind stress, pressure gradient and Coriolis term. Bottom stress typically plays a secondary role. Nonetheless, it was shown that small cross-shore bottom stresses could significantly affect the cross-shore pressure gradient despite being a small term in the total balance.

\subsection{Variation of the Drag Tensor}

The drag tensor was formulated so as to have a magnitude, $r$, which is used by the depthaveraged model to scale the current speed to the bottom stress magnitude and an angle, $\theta$, which is used to rotate the depth-averaged velocity to the direction of the bottom stress. The DRM described in section 2.2 was used to estimate drag tensors for the DAM. This was done by first calculating velocity profile and bottom stress estimates. The profile was then integrated to yield a depth-averaged velocity estimate. Finally, the bottom stress magnitude was divided by the depth-averaged velocity magnitude to obtain $r$, while the difference in their directions yielded $\theta$.

The DRM was formulated in such a way that it produced drag tensor estimates efficiently while retaining a great deal of physics. The only simplification required to keep the DRM quasi-analytical was that the local time-derivatives were assumed depth-independent. This

limits temporally-resolvable processes to those with periods much greater than the diffusion time scale.

Comparison of bottom stress estimates from the DRM and DAM in section 3.1 showed adequate agreement. However, agreement for the large value of $u_{* s} / f h$ was a bit better. This may have been a reflection of the shorter update period providing improved temporal resolution and/or the assumption of barotropic acceleration terms being more appropriate in the shallow case. In addition, because the update frequency was based on an estimate of the diffusion time scale and because updating introduced small numerical waves, separation of time scales was 
important so that the numerical response was not confused with the physical response.

The temporal behavior of $r$ and $\theta$ was reported extensively for three of the four scenarios (sections $3.1,3.2$ and 3.4). In all three, they showed a great deal of time dependence. The overall spin-up and wavelike adjustment time scales for the constant wind cases were comparable to those of the flow field. With a rotating wind, they exhibited periodic behavior with twice the frequency of the forcing. This is understandable since $r$ is a positive-definite quantity determined by only the magnitudes of the bottom stress and depth-averaged flow which had two maxima per forcing period.

The steady-state value of $r$ was found to be a function of $u_{* s} / f h, z_{0} / h$ and the angle between the wind stress and coastline. The resistance coefficient varied directly with $u_{* s} / f h$ for the constant depth cases in section 3.1. Similarly, the largest values of $r$ were found in shallow water for the three variable depth cases. Although clearly related to cross-shore differences in $u_{* s} / f h$, some of the variation in sections $3.2-3.4$ was also due to variation in $z_{0} / h$. The results of Jenter and Madsen (1989) support this. Variation of $r$ with wind direction was shown in section 3.3 , but found to be too complicated to render it simply predictable.

Steady-state values of $\theta$ were, in general, found to be small. However, their effect on the basic model outputs was significant. $\theta$ was shown to be a function of $u_{* s} / f h, z_{0} / h$ and wind angle. The most significant variation appeared to be with wind angle (section 3.1). Rotating the wind counter-clockwise corresponded to rotating steady-state $\theta$ clockwise. $\theta$ varied most rapidly as the wind became more perpendicular to the shore. The same sense of rotation occured temporally in section 3.4. However, the time of fastest rotation corresponded to times of alongshore winds.

Estimated drag coefficients were calculated by dividing the resistance coefficient by the magnitude of the depth-averaged velocity. This was done to examine the temporal, spatial and steady-state behavior of $C_{d}$. The drag coefficient was found to have as much temporal variability as $r$, despite the fact that $r$ often appears to be correlated with the depth-averaged velocity. This indicates that traditional quadratic drag laws are not able to reproduce adequately the transient relationship between bottom stress and depth-averaged flow.

Unlike $r$, the steady-state value of $C_{d}$ is only a function of $z_{0} / h$. Results from section 3.1 showed that it is not strongly dependent on $u_{* s} / f h$. It was demonstrated in section 3.3 that 
$C_{d}$ does not depend significantly on wind angle either. This is of some importance for choosing a traditional quadratic drag law over a linear one, since it may be easier to estimate values of $z_{0} / h$ a priori for a particular domain than to estimate $u_{* s} / f h$ and wind angles. This result does not exempt traditional quadratic drag laws from problems, however. In addition to the absence of temporal variability described above, traditional drag laws still do not allow for the existence of an angle between the depth-averaged flow and bottom stress.

\subsection{Comparison of Drag Tensor and Traditional Drag Law Results}

Comparisons betweeen the $2 \frac{1}{2}$-D model and a traditional model showed both temporal and spatial differences. This is not surprising since traditional models have temporally- and spatiallyconstant drag laws which restrict the bottom stress always to be in the direction of the depthaveraged flow.

Temporally, the traditional model, in general, spun up more slowly from rest. This was due to the abnormally high friction at early times which was caused by the traditional model having a much larger initial resistance coefficient than the $2 \frac{1}{2}$-D model. Different spin-up times imply different abilities to respond to temporal changes in forcing, and, as was shown in section 3.4, a domain's response time greatly affects its basic variable fields.

Spatially, failure to account for cross-shore variation in $r$ was shown in section 3.2 to restrict the steady-state alongshore depth-averaged flow to be constant across the shelf. Only when $r$ was allowed to vary, could the velocity change. Similarly, differences in steady-state flow field magnitude and direction were demonstrated in section 3.3 when $r$ was held constant.

Clearly, improper specification of the alongshore flow field has significant implications for the cross-shore momentum balance since the Coriolis term is so important and the sea surface slope is so sensitive to changes in the alongshore flow. This was particularly true in this thesis because the domains modelled were all clamped at the offshore boundary. Therefore, the sea surface response at any point on the shelf was an integral function of all of the surface slopes seaward of that point. Results in section 3.2 showed that the steady-state surface profile was strongly affected by the assumed cross-shore variation in $r$. 
In addition to neglecting temporal and spatial variations in $r$, traditional models assume $\theta=0$. This has significant implications because small values of $\theta$ can produce large changes in model output, as was demonstrated in section 3.1 when $2 \frac{1}{2}$ - D and traditional models with identical $r$ values showed $20 \%$ differences in surface height when $\theta$ was just $-6^{\circ}$. Likewise, in section 3.2 , the two models showed significant differences in steady-state cross-shore surface profiles even when $r$ was allowed to vary exactly the same way for both models. The reason for this is that, in these straight coastline cases, no predicted cross-shore bottom stress can be reproduced by a traditional model because $V=0$ at steady-state.

\subsection{Conclusion and Recommendations for Future Work}

The major conclusion of this thesis is that the relationship between the depth-averaged flow and bottom stress is complicated and that traditional drag laws are unable to reproduce many important phenomena. Lack of temporal and spatial variability, as well as restriction of the flow and bottom stress to be collinear is the primary problem. Therefore, introduction of a drag tensor, calculated as it was in this thesis, appears to be a worthwhile modification to depth-averaged circulation models.

The results of this thesis point toward areas for future consideration. Primary among these is verification of the $2 \frac{1}{2}$-D model using actual measurements of wind-driven coastal flow fields. The functional dependencies of the drag tensor indicate that proper specification of the fields of $u_{* s} / f h, z_{0} / h$ and wind direction will be critical for good model agreement. Consequently, this implies a need for good meteorological forcing and bottom roughness information. The latter will require development of a formulation for $z_{0}$ which allows it to be determined by, among other variables, the bottom type, bottom configuration and wave climate. 


\section{Bibliography}

[1] J. R. Baker and T. F. Jordan. Vertical structure of time-dependent flow for viscosity that depends on both depth and time. J. Phys. Oceanogr., 11:1673-1674, 1981.

[2] J. R. Baker and T. F. Jordan. Vertical structure of time-dependent flow in a well-mixed fluid with turbulent boundary layers at the bottom and top. J. Phys. Oceanogr., 10:16911694, 1980.

[3] R. C. Beardsley and D. B. Haidvogel. Model studies of the wind-driven transient circulation in the Middle Atlantic Bight. Part 1: Adiabatic boundary conditions. J. Phys. Oceanogr., 11:355-375, 1981.

[4] A. F. Blumberg and H. J. Herring. Circulation Modelling Using Curvilinear Coordinates. Technical Report 81, DYNALYSIS of Princeton, Princeton, NJ, 1983.

[5] A. F. Blumberg and G. L. Mellor. A description of a three-dimensional coastal ocean circulation model. In N. S. Heaps, editor, Three-Dimensional Coastal Ocean Models, pages 1-16, American Geophysical Union, 1987.

[6] A. F. Blumberg and L. Oey. Modelling circulation and mixing in estuaries and coastal oceans. Advances in Geophysics, 28A:525-547, 1985.

[7] K. F. Bowden. Note on wind drift in a channel in the presence of tidal currents. Proc. Roy. Soc. London, 219:426-446, 1953.

[8] C. L. Bretschneider. Storm surges. Advances in Hydroscience, 4:341-418, 1967.

[9] J. A. Businger and S. P. S. Arya. Height of the mixed layer in the stably stratified planetary boundary layer. Adv. Geophys., 18A:73-92, 1974. 
[10] J. A. Carton. Coastal circulation caused by an isolated storm. J. Phys. Oceanogr., 14:114$124,1984$.

[11] S. C. Chao. Forced shelf circulation by an alongshore wind band. J. Phys. Oceanogr., 11:1325-1333, 1981.

[12] D. C. Chapman. Numerical treatment of cross-shelf open boundaries in a barotropic coastal ocean model. J. Phys. Oceanogr., 15(8):1060-1075, 1985.

[13] H. Charnock and J. Crease. Recent advances in science. Sci. Prog., 45:494-511, 1957.

[14] J. H. Churchill and G. T. Csanady. Near-surface measurements of quasi-Lagrangian velocities in open water. J. Phys. Oceanogr., 13:1669-1680, 1983.

[15] F. H. Clauser. The turbulent boundary layer. Adv. Appl. Mech., 4:1-51, 1956.

[16] G. T. Csanady. Circulation in the Coastal Ocean. D. Reidel Publishing Company, 1984.

[17] A. M. Davies. On extracting current profiles from vertically integrated numerical models. Coastal Engineering, 11:445-477, 1987.

[18] V. W. Ekman. On the influence of the earth's rotation on ocean-currents. Arkiv för Matematik, Astronomi Och Fysik, 2(11):1-53, 1905.

[19] The Storm Surge Problem and Possible Effects of Sea Level Changes on Coastal Flooding in the Bay of Bengal, 1987.

[20] G. Z. Forristal. Three-dimensional structure of storm-generated currents. J. Geophys. Res., 79(18):2721-2729, 1974.

[21] W. D. Grant and O. S. Madsen. Combined wave and current interaction with a rough bottom. J. Geophys. Res., 84(C4):1797-1808, 1979.

[22] W. D. Grant and O. S. Madsen. The continental-shelf bottom boundary layer. Ann. Rev. Fluid Mech., 18:265-305, 1986.

[23] W. D. Grant and O. S. Madsen. Moveable bed roughness in unsteady oscillatory flow. J. Geophys. Res., 87(C1):469-481, 1982. 
[24] P. Groen and G. W. Groves. Surges. In M. N. Hill, editor, The Sea, chapter 7, pages 611646, John Wiley \& Sons, 1962.

[25] N. S. Heaps. Storm surges, 1967-1982. Geophys. J. R. astr. Soc., 74:331-376, 1983.

[26] N. S. Heaps. Storm surges, oceanography and marine biology. Mar. Biol. Ann. Rev., 5:11-47, 1967.

[27] N. S. Heaps. A two-dimensional numerical sea model. Philos. Trans. R. Soc. London, 275:93-137, 1969.

[28] N. S. Heaps, J. M. Huthnance, J. E. Jones, and J. Wolf. Modelling of storm-driven shelf waves north of Scotland-i. idealized models. Continental Shelf Research, 8(11):1187-1210, 1988.

[29] N. S. Heaps and J. E. Jones. Storm surge computations for the Irish Sea using a threedimensional numerical model. Mem. Sos. R. Sci. Liège Collect., 6(7):289-333, 1975.

[30] F. B. Hildebrand. Advanced Calculus for Applications. Prentice-Hall Inc., Englewood, NJ, 2nd edition, 1976.

[31] B. M. Jamart and J. Ozer. Comparison of 2-d and 3-d models of the steady wind-driven circulation in shallow waters. Coastal Engineering, 11:393-413, 1987.

[32] C. P. Jelesnianski. A numerical calculation of storm tides induced by a tropical storm impinging on a continental shelf. Monthly Weather Review, 93(6):343-358, 1965.

[33] C.P. Jelesnianski. Bottom stress time-history in linearized equations of motion for storm surges. Monthly Weather Review, 98(6):462-478, 1970.

[34] H. L. Jenter. The One-Dimensional Dynamics of a Steady Wind Forced Water Column in the Presence of Surface Gravity Waves. Master's thesis, Massachusetts Institute of Technology, Cambridge, MA, 1987.

[35] H. L. Jenter and O. S. Madsen. Bottom stress in wind-driven depth-averaged coastal flows. 1989. accepted J. Phys. Oceanog. 
[36] B. Johns and T. Oguz. Turbulent energy closure schemes. In N. S. Heaps, editor, Three Dimensional Coastal Ocean Models, pages 17-39, American Geophysical Union, 1987.

[37] T. F. Jordan and J. R. Baker. Vertical structure of time-dependent flow dominated by a friction in a well-mixed fluid. J. Phys. Oceanogr., 10:1091-1103, 1980.

[38] J. Kielmann and Z. Kowalik. A bottom stress formulation for storm surge problems. Oceanol. Acta, 3(1):51-58, 1980.

[39] R. F. Kossik, P. S. Gschwend, and E. E. Adams. Tracing and Modeling Pollutant Transport in Boston Harbor. Technical Report MITSG 86-16, Massachusetts Institute of Technology, Cambridge, MA 02139, 1986.

[40] H. Lamb. Hydrodynamics. Cambridge University Press, London and New York, 6th edition, 1953.

[41] J. W. Lavelle and H. O. Mofjeld. Effects of time-varying viscosity on oscillatory turbulent channel flow. J. Geophys. Res., 88(C12):7607-7616, 1983.

[42] O. S. Madsen. A realistic model of the wind-induced Ekman boundary layer. J. Phys. Oceanogr., 7:248-255, 1977.

[43] G. L. Mellor and T. Yamada. A hierarchy of turbulence closure models for planetary boundary layers. J. Atmos. Sci., 31:1791-1806, 1974.

[44] F. Mesinger and A Arakawa. Numerical Methods Used in Atmospheric Models. Technical Report, WMO-ICSU, Global Atmospheric Research Programme (GARP), 1976.

[45] H. O. Mofjeld. Depth dependence of bottom stress and quadratic drag coefficient for barotropic pressure-driven currents. J. Phys. Oceanogr., 7:1658-1669, 1988.

[46] T. S. Murty. Storm Surges-Meteorological Ocean Tides. Technical Report 212, Canadian Department of Fisheries and Oceans, 1984.

[47] J. Nezu and W. Rodi. Open-channel flow measurements with a laser doppler anemometer. Journal of Hydraulic Engineering, 112(5):335-355, 1986. 
[48] J. C. J. Nihoul. Three-dimensional model of tides and storm surges in a shallow well-mixed continental sea. Dynam. Atmos. Oceans, 2:29-47, 1977.

[49] J. Noye and M. Stevens. A three-dimensional model of tidal propagation using transformations and variable grids. In N. S. Heaps, editor, Three-Dimensional Coastal Ocean Models, pages 41-69, American Geophysical Union, 1987.

[50] J. E. Overland, H. O. Mofjeld, and C. H. Pease. Wind-driven ice drift in a shallow sea. J. Geophys. Res., 89(C4):6525-6531, 1984.

[51] G. W. Platzman. Two-dimensional free oscillations in natural basins. J. Phys. Oceanogr., 2(2):117-138, 1972.

[52] R. O. Reid. Modification of the Quadratic Bottom Stress Law for Turbulent Channel Flow in the Presence of Surface Wind Stress. Technical Report 93, Beach Erosionn Board, Corps of Engineers, 1957.

[53] J. G. Richman, R. A. de Szoeke, and R. E. Davis. Measurements of near-surface shear in the ocean. J. Geophys. Res., 92(C3):2851-2858, 1987.

[54] Oceans 87. Modelling of meteorologically forced currents on the Scotian Shelf, 1987.

[55] O. H. Shemdin. Wind-generated current and phase speed of wind waves. J. Phys. Oceanogr., 2:411-419, 1972.

[56] R. P. Signell and W. R. Geyer. Numerical simulation of tidal dispersion around a coastal headland. 1989. submitted to jgr.

[57] J. D. Smith. The Sea. Volume 6, Wiley Interscience, New York, 1977.

[58] J. D. Smith and C. E. Long. The effect of turning in the bottom boundary layer on continental shelf sediment transport. Mémoires Société Royale des Sciences de Liège, $6^{e}: 369-396,1976$.

[59] R. L. Soulsby. Physical Oceanography of Coastal and Shelf Seas. Elsevier Oceanography Series, Elsevier, Amsterdam, 1983. 
[60] M. W. Stacey, S. Pond, and P. H. LeBlond. A wind-forced Ekman spiral as a good statistical fit to low-frequency currents in a coastal strait. Science, 233:470-472, 1986.

[61] E. A. Terray, A. J. Williams 3rd, B. H. Brumely, and Y. C. Agrawal. Abstract: results from the WAVES upper layer mixing study. Eos, Trans. Am. Geophys. Union, 68(44):1313, 1987.

[62] J. H. Thomas. A theory of steady wind-driven currents in shallow water with variable eddy viscosity. J. Phys. Oceanogr., 5:136-142, 1975.

[63] P. Welander. Numerical prediction of storm surges. Advances in Geophysics, 8:315-379, 1961.

[64] P. Welander. Wind action on a shallow sea: some generalizations of Ekman's theory. Tellus, 9:45-52, 1957.

[65] B. W. Wilson. Seiches. Adv. Hydrosci., 8:1-94, 1972.

[66] J. Wu. Wind-induced drift currents. J. Fluid Mech., 68:49-70, 1975. part 1.

[67] J. Wu. Wind-stress coefficients over sea surface from breeze to hurricane. J. Geophys. Res., 87(c12):9704-9706, 1982. 